\title{
Modeling cachexia-associated muscle alterations with patient-derived pancreatic tumor organoids
}

Citation for published version (APA):

Vaes, R. (2021). Modeling cachexia-associated muscle alterations with patient-derived pancreatic tumor organoids. [Doctoral Thesis, Maastricht University]. ProefschriftMaken.

https://doi.org/10.26481/dis.20210520rv

Document status and date:

Published: 01/01/2021

DOI:

10.26481/dis.20210520rv

Document Version:

Publisher's PDF, also known as Version of record

\section{Please check the document version of this publication:}

- A submitted manuscript is the version of the article upon submission and before peer-review. There can be important differences between the submitted version and the official published version of record.

People interested in the research are advised to contact the author for the final version of the publication, or visit the DOI to the publisher's website.

- The final author version and the galley proof are versions of the publication after peer review.

- The final published version features the final layout of the paper including the volume, issue and page numbers.

Link to publication

\footnotetext{
General rights rights.

- You may freely distribute the URL identifying the publication in the public portal. please follow below link for the End User Agreement:

www.umlib.nl/taverne-license

Take down policy

If you believe that this document breaches copyright please contact us at:

repository@maastrichtuniversity.nl

providing details and we will investigate your claim.
}

Copyright and moral rights for the publications made accessible in the public portal are retained by the authors and/or other copyright owners and it is a condition of accessing publications that users recognise and abide by the legal requirements associated with these

- Users may download and print one copy of any publication from the public portal for the purpose of private study or research.

- You may not further distribute the material or use it for any profit-making activity or commercial gain

If the publication is distributed under the terms of Article $25 \mathrm{fa}$ of the Dutch Copyright Act, indicated by the "Taverne" license above, 


\section{Modeling cachexia-associated muscle alterations with patient-derived pancreatic tumor organoids}

Rianne D.W. Vaes 
The work presented in this dissertation was performed at the Department of Surgery, NUTRIM School of Nutrition and Translational Research in Metabolism at Maastricht University

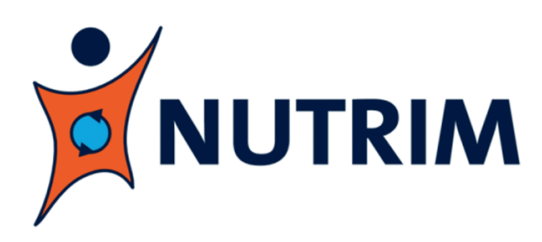

(c) Copyright Rianne Vaes, Maastricht 2021

ISBN: $\quad$ 978-94-6423-221-9

Printed by: ProefschriftMaken || www.proefschriftmaken.nl

Cover design by: ProefschriftOntwerp || Bregje Jaspers || www.proefschriftontwerp.nl

All rights reserved. No part of this publication may be reproduced, distributed, or transmitted in any form or by any means, including photocopying, recording, or other electronic or other mechanical methods, without the prior permission in writing from the author. 


\title{
Modeling cachexia-associated muscle alterations with patient-derived pancreatic tumor organoids
}

\author{
PROEFSCHRIFT \\ Ter verkrijging van de graad van doctor aan de Universiteit Maastricht, \\ op gezag van de Rector Magnificus, Prof. dr. Rianne M. Letschert \\ volgens het besluit van het College van Decanen, \\ in het openbaar te verdedigen \\ op donderdag 20 mei 2021 om 14.00 uur
}

door

Rianne D.W. Vaes 
Promotor

Prof. dr. S.W.M. Olde Damink

\section{Copromotor}

Dr. S.S. Rensen

Beoordelingscommissie

Prof. dr. P.J. Peters (voorzitter)

Prof. dr. A.M.W.J. Schols

Prof. dr. D.M.A.E. Jonkers

Prof. dr. T. Cramer (RWTH University Hospital Aachen, Germany)

Dr. M-L. Bochaton-Piallat (University of Geneva, Switzerland) 


\section{TABLE OF CONTENTS}

Chapter 1 General introduction and outline of the thesis

Chapter 2 Generation and initial characterization of novel tumor organoid models to study human pancreatic cancer-induced cachexia

Chapter 3 Human pancreatic tumor organoid-derived factors enhance 69 myogenic differentiation

Chapter 4 A novel human cell culture model to study visceral smooth 95 muscle phenotypic modulation in health and disease

Chapter 5 Pancreatic tumor organoid-derived factors of patients with cachexia promote phenotypic modulation and proliferation of visceral smooth muscle cells

Chapter 6 Intestinal smooth muscle aberrations in pancreatic cancer patients with sarcopenia

Chapter 7 General discussion 



\section{CHAPTER 1}

General introduction and outline of the thesis 


\section{Pancreatic cancer}

Pancreatic cancer is a major cause of cancer-associated mortality, with a dismal overall prognosis that has remained relatively unchanged for many decades (1). According to the GLOBOCAN 2018 estimate, pancreatic cancer is currently the $7^{\text {th }}$ leading cause of cancerrelated deaths worldwide (2). In addition, the 5-year overall survival rate of pancreatic cancer patients ranges between 2 and $9 \%$. These global estimates are also representative for the current disease burden of pancreatic cancer in the Netherlands. The number of new cases of pancreatic cancer in 2017 in the Netherlands was 2,460 with a general five-year survival of less than $9 \%$, accounting for the $8^{\text {th }}$ leading cause of cancer-related deaths (3). In the past decades, the number of Dutch patients diagnosed with pancreatic cancer increased from 1,400 to approximately 2,400 new diagnoses each year, and this number is expected to continue to rise in the coming years. In contrast, the survival rates of pancreatic cancer have only minimally improved in recent decades. These numbers emphasize the need for a better understanding of the disease, which ultimately will help to improve the overall survival rate of with pancreatic cancer.

Pancreatic ductal adenocarcinoma (PDAC) is the most common malignancy of the pancreas (commonly and here referred to as pancreatic cancer), comprising more than $90 \%$ of all malignant pancreatic neoplasms. PDAC is an exocrine tumor that develops from cells that constitute the exocrine gland and ducts of the pancreas. The exocrine acinar cells produce digestive enzymes, which are secreted into a series of progressively larger ducts, that eventually join to form the main pancreatic duct. The main pancreatic duct runs the length of the pancreas and drains the fluid produced by the acinar cells into the first part of the small intestine, also called the duodenum. In the duodenum, these enzymes digest carbohydrates, fats, and proteins. The molecular pathology of pancreatic cancer is dominated by mutations in KRAS, which are detected in $>90 \%$ of tumors. The KRAS gene usually harbors missense mutations that encode single amino acid substitutions predominantly at one of three mutational hot spots: glycine-12 (G12), glycine-13 (G13), or glutamine-61 (Q61) (4). Mutations at one of these hotspots result in constitutively active RAS protein and overstimulation of effector signaling pathways that drive cancer growth (4). Additional well-characterized gene mutations that predominate in pancreatic cancer include those in TP53, SMAD4, and CDKN2A, of which TP53 is mutated in $>50 \%$ of the patients (5).

\section{Diagnosis and treatment of pancreatic cancer}

Recent advances in the early detection and/or the introduction of improved treatment modalities have resulted in substantially improved survival rates of many cancer types. In contrast, pancreatic cancer usually remains undetected in the early stage of tumor development due to a lack of early disease-specific symptoms as well as sensitive and 
specific tumor markers, and difficulties in imaging early-stage tumors. As a result, the tumor has frequently already progressed to a locally advanced or metastasized stage at the time of diagnosis.

Nowadays, surgical removal of the pancreatic tumor is still the only potential cure. Unfortunately, only $15-20 \%$ of the patients are eligible for surgery (6). Patients that have an unresectable tumor have either metastatic disease at the time of diagnosis or the tumor has locally invaded healthy tissue. Initially non-resectable or borderline resectable tumors may be down-staged to resectable tumors after neoadjuvant treatment, including radiation and chemotherapy (7). Alternative treatment modalities include palliative chemotherapy. For many years, gemcitabine has been the standard therapy in patients with pancreatic cancer, but recently, overall survival has been significantly improved using combination therapies. These therapies include a combination of gemcitabine with erlotinib, nabpaclitaxel, or a combination of fluorouracil, leucovorin, irinotecan, and oxaliplatin (FOLFIRINOX) (8). Nevertheless, these treatment regimens still only modestly increase the overall survival of pancreatic cancer patients.

\section{Cancer cachexia}

While the diagnosis and treatment of pancreatic cancer patients still remain major challenges, the cachexia syndrome, which is present in up to $70 \%$ of pancreatic cancer patients, is another relatively underestimated and underrecognized medical problem that significantly contributes to the poor survival of these patients (9-11). The term cachexia originates from the Greek words "kakos" and "hexis", and literally means "bad condition". It is a severe wasting condition that is associated with reduced physical function (12), reduced tolerance to anticancer treatment regimens (13), and a marked reduction in quality of life (QoL) $(14,15)$.

\section{Definition and classification of cancer cachexia}

Over the years, various definitions of this complex condition have been published incorporating several of its key aspects, including weight loss, fatigue, anorexia, and inflammation (16). However, establishing a clinically meaningful definition for use in clinical practice has been challenging. Traditionally, cachexia has been defined by a specified percentage of weight loss over time. Yet, assessment of weight loss alone does not reflect the complete scope of pathophysiological changes or the clinical impact of cachexia (17). In 2011, international experts agreed upon a consensus definition in which cancer cachexia was defined as a "multifactorial syndrome characterized by an ongoing loss of skeletal muscle mass (with or without loss of fat mass) that cannot be fully reversed by conventional nutritional support and leads to progressive functional impairment" (18). In the present definition, cancer cachexia is described to evolve through a spectrum that can generally be subdivided into three clinically relevant stages, namely pre-cachexia, cachexia, and 
refractory cachexia. In the pre-cachexia stage, early metabolic alterations result in minor involuntary body weight loss. Although interventions within this stage are most likely to be effective, the pre-cachexia stage frequently remains unrecognized by clinicians due to a lack of definite clinical signs. As a consequence, pre-cachexia often progresses towards the cachexia stage. Patients who lose more than $5 \%$ of their normal weight over the past 6 months in the absence of simple starvation (i.e. inadequate intake of protein and/or calories), or have a BMI less than $20 \mathrm{~kg} / \mathrm{m}^{2}$ and ongoing weight loss of more than $2 \%$, or have a low skeletal muscle mass (also known as sarcopenia) and ongoing weight loss of more than $2 \%$ are classified as having cachexia (18). The refractory cachexia stage is characterized by severe muscle wasting, ongoing catabolism, and a low performance status. These patients are no longer responsive to anti-cancer treatment and active management of weight-loss is not effective.

\section{Assessment of cancer cachexia}

Although experts in the field have agreed upon a consensus definition, the diagnosis of cancer cachexia remains a challenge both in research and in clinical practice $(19,20)$. For example, involuntary weight loss, which is a key feature of cancer cachexia, can also be the consequence of malnutrition, starvation, malabsorption, and/or age-related loss of muscle mass (21). It is important to recognize that cachexia differs from these conditions as it involves metabolic alterations resulting in muscle and fat wasting that cannot be fully reversed by nutritional support (20). However, body weight measurements alone cannot discriminate between cachexia and these other conditions. Also, body weight measurements cannot differentiate between skeletal muscle and fat tissue mass and may therefore underestimate muscle weight loss in overweight or obese patients. Furthermore, some cancer patients may even gain weight because of a growing tumor mass or by abnormal accumulation of fluid within the body (i.e. edema, ascites), but still experience undetected muscle tissue loss (22). Therefore, a broad range of assessments are required for adequate characterization of the cachexia status of each patient $(16,17)$. The diagnosis of cancer cachexia is further complicated by the heterogeneity in the clinical presentation of cachexia, difficulties in consistent diagnosis of the disease at early stages, the advanced age of many patients, and the complexity of the multimodal approach needed to treat cachexia, which have hampered efforts towards a standardized and effective treatment (16).

There is a wide range of tools and measurements that may be of interest when assessing the actual cachexia status of cancer patients. These measurements provide support to diagnose and classify cancer cachexia in clinical practice and research settings and reveal additional insight into nutritional status, physical performance, body composition, and immune status. 
Nutritional status. In recent years, increasing attention has been paid to the nutritional status of cancer patients. Patients with cancer are at particularly high risk of the development of malnutrition, which is associated with higher mortality rates, greater functional decline, poorer quality of life, and longer recovery times (23-25). Therefore, nutritional support has become an important aspect of multimodal cancer care. To identify and diagnose malnutrition in cancer patients, several nutritional screening tools are available, including the patient-generated subjective global assessment (PG-SGA), the mini nutritional assessment (MNA), and the malnutrition universal screening tool (MUST). The PG-SGA is a validated screening tool for malnutrition in hospitalized patients that has been adapted for cancer patients $(26,27)$. The PG-SGA incorporates questions for patients regarding weight history, caloric intake, and functional status and requires additional assessments by healthcare professionals, including comorbid conditions, fever, medications, and a detailed physical examination focusing on fat and muscle mass (28). Moreover, a recent study by Martins et al. demonstrated that the PG-SGA nutritional score was predictive for cancer cachexia in patients with head, neck, and abdominal cancer (29). The MNA is another validated screening tool that is designed to be completed within 10 minutes and which can therefore be easily applied for the early detection of malnutrition by health care professionals in elderly patients across various settings, including outpatient clinics, nursing homes, and hospitals $(30,31)$. MUST is a screening tool to identify adults who are malnourished, at risk of malnutrition, or obese; it incorporates a score based on BMI, history of weight loss, and disease comorbidities (32). Tools like the PG-SGA, MNA, and MUST are relatively inexpensive and easy to implement in the clinical setting.

Body composition. Cancer cachexia is associated with marked alterations in body composition with a loss of lean muscle mass with or without loss of adipose tissue. As already indicated, body weight measurements alone can be misleading as they cannot discriminate between skeletal mass and fat tissue mass. Therefore, accurate measurements of body composition are essential to assess the actual cachexia status of patients. The most commonly used body composition assessments in cancer patients include anthropometric methods and computed tomography (CT) imaging analysis (33). Anthropometric measurements include height, body weight, BMI, skinfold thickness, and arm circumferences. The skinfold thickness is a measure of subcutaneous fat mass and can be used to estimate the total amount of body fat (34-36). This method is based on the hypothesis that fat is equally distributed over the body. The triceps is the most reliable location to assess skinfold thickness because edema is not often seen in the upper arm. The upper arm circumference is, together with the triceps skinfold, a measure of the quantity of fat and muscle mass in the body. Additionally, the upper arm fat mass, also measured by upper arm circumference and the triceps skinfold, provides a better assessment of the total body fat mass compared to a single skinfold measurement. If the patient is cachectic, the thickness of the triceps skinfold and the area of the upper arm are decreased. Repeated 
measurements can therefore be indicative of changes in body composition and the nutritional status of the patient. Frisancho et al. have published tables with percentiles that allow to compare the measured values with reference values that have been obtained from a general population (37).

Nowadays, CT imaging is the gold standard for body composition $(33,38)$. Due to its high precision and specificity for discriminating individual tissue compartments, CT imaging can discriminate between adipose tissue, bone, organs, and muscle including the degree of fat infiltration. To assess body composition, CT scans are evaluated at a standard skeletal landmark, often the third lumbar vertebra (L3) since it strongly correlates with total body skeletal muscle area (39). The muscle cross-sectional area can be indexed for height to derive estimates of whole-body muscularity (skeletal muscle index, SMI), which in turn has been shown to be of prognostic value in terms of patient survival and postoperative morbidity $(22,40,41)$. CT imaging can also be used to assess muscle quality, by measuring the radio-density of skeletal muscle tissue compartments in Hounsfield Units. In a prospective study involving 199 patients with cancer in the head of the pancreas, we recently showed that medium or low muscle radiodensity was associated with poorer survival (42). Similar findings have been reported in patients with endometrial cancer and patients with palliative pancreatic cancer $(43,44)$. Recognizing the increasing use of CT imaging for cancer staging, it is an attractive assessment tool for body composition analysis in cancer patients.

Functional performance. Functional impairment and reduced physical performance are additional key factors associated with the cachexia syndrome (25). Whereas the decrease in muscle mass can be relatively small, there may be a significant decrease in muscle performance (12). Therefore, adequate evaluation of the patients' performance status is important for the assessment of the cachexia status. In routine clinical practice, the performance status is commonly estimated using the Eastern Cooperative Oncology Group (ECOG) score, which is a doctor-reported outcome. The ECOG performance status, first published in 1982, is a scale used to assess how a disease affects the daily living abilities of the patient and how the disease is progressing (45). However, it is likely that the patients true performance status is underestimated or overestimated and therefore a more objective evaluation of the patient's performance status is of added value in the evaluation of the cachexia status. Handgrip strength is a simple and quick measure of muscle function that can easily be implemented in routine clinical practice. The maximal handgrip strength is an indication of peripheral muscle function and is related to the total amount of muscle mass in the body. A low handgrip strength has previously been associated with a low muscle mass and worse overall survival rates $(46,47)$.

Biological markers. Systemic inflammation has an important role as a key driver of energy imbalance and muscle wasting in cancer cachexia $(48,49)$. Laboratory data collected during 
routine clinical care can reveal relevant insight into the immune status of the patient and can be easily incorporated in the diagnosis of cancer cachexia (33). For example, tumor- and host-derived pro-inflammatory cytokines can trigger a systemic inflammatory response and cause an acute phase response with increased C-reactive protein (CRP) and decreased albumin levels $(50,51)$. The most widely accepted index to characterize systemic inflammation is the modified Glasgow Prognostic Score (mGPS), which combines the indicators of elevated CRP and decreased plasma albumin (52). The mGPS has been validated and reported to correlate with poor nutritional status, weight loss, and a decreased response to chemotherapy $(53,54)$. In addition, a high neutrophil/lymphocyte ratio (NLR) (>3.5) has been associated with progressive disease, weight loss, and decreased survival in cancer patients $(55,56)$.

\section{Cancer cachexia-induced muscle wasting}

The involuntary loss of muscle mass in cancer cachexia mainly concerns skeletal muscle, which accounts for $40 \%$ of total body weight. Under physiological conditions, muscle mass remains relatively constant in the absence of anabolic and catabolic stimuli. In cancer cachexia-related muscle wasting, it is widely assumed that both tumor-and host-derived catabolic stimuli and pro-inflammatory cytokines (e.g. TNF- $\alpha$, IL-1, and IL-6) directly target skeletal muscle (57). These factors bind to their respective cell surface receptors to promote muscle atrophy via the regulation of anabolic and catabolic pathways (58-60). Ultimately, muscle atrophy occurs as the result of a disturbed balance between anabolic and catabolic processes (61). Muscle types that comprise the human muscular system include skeletal muscle, cardiac muscle, and smooth muscle. Cachexia-associated alterations have been observed in skeletal and cardiac muscle, but it is unknown whether these changes also occur in smooth muscle. In the next section, the current knowledge on cancer cachexia-associated muscle wasting will be discussed.

\section{Skeletal muscle atrophy}

Skeletal muscle mass is largely dependent upon the muscle myosin fiber content. Skeletal muscle fibers are extremely adaptable and can meet the varying demands of muscle by responding to changes in environmental cues in several ways (62). The myofibrillar protein myosin is the most abundant protein and produces the force necessary for a variety of cellular movements by interacting with actin filaments. Muscle fibers can generally be classified based on their contraction speed, i.e. slow-twitch (type I) or fast-twitch (type Ila, type IIx, and type IIb), and based on the metabolic pathway that is used to degrade glycogen (i.e. oxidative or glycolytic). Specifically, muscle fiber type is defined by four myosin heavy chain (MyHC) isoforms, the oxidative MyHC fibers MyHC-I (MYH7) and MyHC-Ila, and the glycolytic fibers MyHC-IIx (MYH1) and MyHC-Ilb (MYH4). Skeletal muscle proteins are continuously being remodeled through the simultaneous processes of muscle protein 
synthesis and muscle protein breakdown. The daily difference between muscle protein synthesis and breakdown defines the net protein balance, which determines overall skeletal muscle mass.

Muscle protein synthesis is predominantly regulated by the insulin-like growth factor-1/phosphoinositide-3kinase/Akt/-mammalian target of rapamycin (IGF1/PI3K/Akt/mTOR) pathway (Fig. 1). IGF-1 is an anabolic growth factor; binding of IGF-1 to its receptor triggers activation of several intracellular kinases, including PI3K which, in turn, causes Akt activation. Direct and indirect downstream targets of Akt include the mammalian target of rapamycin (mTOR), ribosomal protein S6 kinase beta 1 (S6K1, also known as p70S6K), and the eukaryotic translation initiation factor 4E (elF4E)-binding protein 1 (4EBP1), which are all key regulatory proteins involved in translation and protein synthesis (63, 64). mTORC1 serves as a critical regulatory point for anabolic stimuli, including growth factors, nutrients, and mechanical load (65). Upon activation, mTORC1 directly phosphorylates 4E-BP1 and p70S6K to promote protein synthesis (66). The hyperphosphorylation of 4E-BP1 prevents binding to elF4E and the formation of the 4E-BP1elF4E complex, resulting in the assembly of the elF4E complex and translation initiation

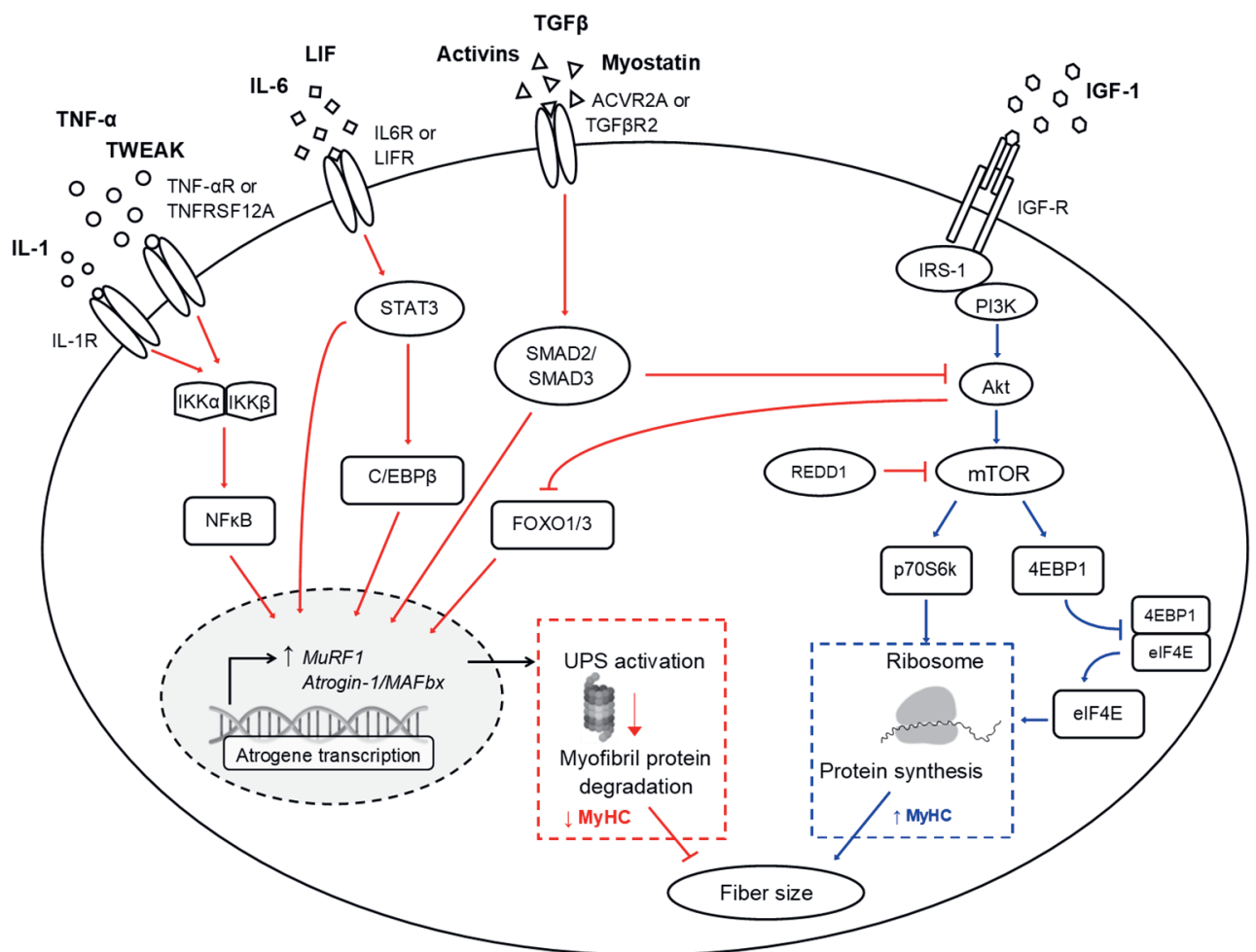

Figure 1: Signaling pathways involved in tumor-induced skeletal muscle atrophy. Skeletal muscle proteins are continuously being remodeled through the simultaneous processes of muscle protein synthesis and muscle protein breakdown. 
(Fig. 1). Prior studies with tumor-bearing mice have revealed that cachexia-related skeletal muscle loss is associated with decreased rates of muscle protein synthesis (67-71). However, in human cancer cachexia, the modulation of protein synthesis rates is less clear and contradictory results have been reported. Whereas Emery et al. detected reduced protein synthesis rates in the skeletal muscle of cachectic cancer patients (72), MacDonald et al. did not report differences in myofibrillar protein synthesis rates between healthy, weight stable subjects, and weight losing patients with upper gastrointestinal cancer (73). Despite our limited understanding of cachexia-induced protein synthesis suppression in cancer patients, it is suggested that decreased protein synthesis rates can be caused by a failure of the normal stimuli for muscle protein synthesis and/or in the supply of amino acids and energy for net protein deposition to occur (74).

In cancer cachexia, a decrease in skeletal muscle mass can result from both decreased protein synthesis rates and increased protein degradation rates. Muscle atrophy occurs when the overall rates of protein degradation rates exceed the protein synthesis rates. Studies in animal models of cancer cachexia, as well as in cancer patients, suggest that the ubiquitin-proteasome (UPS) pathway plays a predominant role in the degradation of myofibrillar proteins (Fig. 1) $(58,75-77)$. Proteins to be degraded by the UPS are first conjugated to multiple ubiquitin molecules through a reaction sequence consisting of a series of ubiquitin ligases. Three distinct ubiquitin ligases are involved in the ubiquitination of these ubiquitin-labeled proteins, three distinct ubiquitin ligases are involved: an E1 ubiquitin-activating enzyme, an E2 ubiquitin-conjugating enzyme, and an E3 ubiquitinligating enzyme. In cancer cachexia, the muscle-specific E3-ubiquitin ligases muscle atrophy F box (MAFbx)/atrogin-1 and muscle RING finger 1 (MuRF1) have been reported to be upregulated under atrophy-inducing conditions $(78,79)$. For example, Bodine et al. showed that overexpression of Atrogin-1/MAFbx in myotubes induced atrophy, while mice deficient in either Atrogin-1/MAFbx or MuRF1 were found to be resistant to atrophy (78). Furthermore, in experimental models of cancer cachexia, myosin heavy chain has been confirmed to be a substrate of the E3-ubiquitin ligase MuRF1 $(58,80,81)$.

The increased expression of these skeletal muscle specific E3-ubiquitin ligases is largely under the control of the transcription factors forkhead box protein 01 (FOXO1) and FOXO3, whose activities are post-translationally regulated $(82,83)$. Under physiological conditions, Akt phosphorylates the FOXO proteins and thereby prevents their translocation into the nucleus. However, in cachexia, AKT activity is often suppressed, resulting in the dephosphorylation and subsequent translocation of the FOXO proteins into the nucleus, which in turn enables the transcription of MuRF1 and Atrogin-1/MAFbx (Fig. 1). Therefore, FOXO proteins are suggested to function as a regulatory node between anabolic and catabolic processes.

In addition, tumor- and host-derived factors have been shown to induce skeletal muscle catabolism. These factors have been primarily identified through in vitro 
experiments and tumor xenograft models and include pro-inflammatory cytokines and members of the transforming growth factor- $\beta$ (TGF $\beta$ ) superfamily, including activins, myostatin and TGF $\beta$. Pro-inflammatory mediators that promote skeletal muscle catabolism include interleukin-6 (IL-6), IL-1, tumor necrosis factor alpha (TNF- $\alpha$ ), leukemia inhibitory factor (LIF), and TNF-related weak induced of apoptosis (TWEAK; also known as TNFSF12). Binding of these factors to their respective cell surface receptor activates selective transcription factors such as nuclear factor- $\mathrm{kB}$, signal transducer and activator of transcription 3 (STAT3) and CCAAT/enhancer-binding protein- $\beta$ (C/EBP $\beta$ ), which in turn promotes the transcription of the skeletal muscle specific ubiquitin ligases MuRF1 and Atrogin-1 (Fig. 1) (84-88). Subsequently, activation of the UPS results in the ubiquitination and breakdown of myofibrillar proteins.

Although existing in vitro and in vivo models have contributed tremendously to our current understanding of cancer cachexia mechanisms, their suitability for modeling human cancer cachexia may not be optimal. Recently, Talbert et al. showed that cancer-induced muscle wasting in tumor-bearing mice resulted in the typical large increase in expression of MuRF1 and Atrogin-1/MAFbx, whereas no changes in the expression of these genes were observed in muscles from cachectic pancreatic cancer patients (89). Also other studies did not observe increased expression of UPS components in skeletal muscle biopsies from cancer patients (90-93). Therefore, more insight into the underlying pathophysiological mechanisms of human cancer-induced skeletal muscle wasting is essential.

\section{Cardiac muscle}

Similar alterations to those described above for skeletal muscle have recently been observed in the cardiac muscle of cachectic patients $(94,95)$. The loss of cardiac proteins has been shown to be associated with weight loss in cachectic cancer patients. The presence of cachexia-associated cardiac muscle wasting in cancer patients is further supported by cardiac abnormalities, including symptoms that are clinical indicators of chronic heart failure such as fatigue, shortness of breath, and impaired exercise tolerance (96). Increased awareness of cancer- and treatment-induced cardiac abnormalities, i.e. cardio-oncology, is currently a rapidly growing field that intends to improve the quality of life of patients with cancer by preventing and monitoring cardiovascular events.

In animals bearing cachexia-inducing tumors, cardiac alterations including marked fibrosis, disrupted myocardial ultrastructure, and alterations to the composition of contractile proteins, such as troponin I and myosin heavy chain- $\alpha$, have been observed (97). Similar to the mechanisms underlying skeletal muscle atrophy, cardiac wasting seems to be related to increased cardiac muscle proteolysis, which is driven by the UPS. Indeed, loss of cardiac myofibrillar proteins $\mathrm{MyHC}$ and troponin I has been shown to be accompanied by increased protein ubiquitination and higher expression of Atrogin-1/MAFbx and MuRF1 (98). Furthermore, inhibition of NF-KB has been shown to protect against tumor-induced cardiac atrophy in mice (99). This finding reinforces the role of pro-inflammatory cytokines, 
such as IL-1, IL-6, and TNF $\alpha$, in mediating cancer cachexia-associated cardiac dysfunction (48).

Although the real impact of cardiac atrophy remains unclear in the clinical context (100), increasing numbers of studies reveal an association between cachexia and cardiac atrophy in cancer patients. For example, cardiac muscle has been shown to display pronounced atrophy and dysfunction in cachectic individuals with pancreatic, lung, or colorectal cancer $(94,95)$. Recently, Kazemi-Bajestani et al. revealed atrophy of the left ventricular mass in conjunction with losses of fat and skeletal muscle in patients with nonsmall cell lung cancer treated with palliative chemotherapy (100). Although prospective longitudinal studies are needed to assess the full scope of cardiac atrophy, these data suggest that all striated muscles may atrophy similarly in response to tumor- and/or hostderived catabolic factors.

\section{Smooth muscle}

So far, it is not known whether the changes found in atrophying skeletal and cardiac muscle in response to tumor- and host-derived factors also occur in smooth muscle, the third muscle compartment that fulfils a crucial role in human physiology. However, as all muscle types and other target tissues may be similarly targeted by circulating tumor- and hostderived catabolic factors, it is plausible that also smooth muscle will be affected.

Sheets or layers of smooth muscle cells (SMCs) line the wall of various organs and tubes in the body, including blood vessels, stomach, intestines, bladder, airways, and the uterus. Under physiological conditions, these SMC layers enable endured contraction or dilation, resulting in the transport or storage of fluids. In order to fulfill this function, SMCs exhibit a contractile phenotype which is characterized by an elongated spindle-shaped morphology and high expression of a unique repertoire of contractile proteins (101). In contrast to skeletal and cardiac muscle cells that differentiate terminally, mature contractile SMCs retain remarkable plasticity as expressed by their ability to undergo so-called phenotypic modulation in response to injury and disease. During phenotypic modulation of contractile SMCs, loss of contractile proteins is accompanied by morphological alterations resulting in round-shaped SMCs, referred to as epithelioid or rhomboid, as well as increased proliferation rates, high migration rates, and increased synthesis of extracellular matrix (ECM) proteins (101).

SMC plasticity is essential for adaptation of visceral organs to changes in local environmental conditions in health. For example, during pregnancy, uterine SMC hypertrophy and collagen synthesis are induced by ovarian hormones to cope with the stretch induced by the growing fetus and to maintain a quiescent contractile state until delivery $(102,103)$. However, SMC phenotypic plasticity can also contribute to disease. In inflammatory bowel disease, increased TNF- $\alpha$ and IL-1 $\beta$ levels have been shown to reduce intestinal SMC contractility, contributing to intestinal motility problems $(104,105)$. Reduction of the contractile SMC function, and consequently GI-motility problems, may 
contribute to underlying nutritional-related functional complications in cancer cachexia. In line with this hypothesis, Zhou and colleagues identified symptoms of early satiety, diarrhea, and constipation in cachectic cancer patients that could be secondary to GIsmooth muscle dysfunction (106). Nevertheless, no studies have been reported so far on smooth muscle in the context of cancer cachexia.

\section{Experimental models of cancer-induced cachexia}

Most of our current understanding of the mechanisms driving cachexia-associated muscle alterations is derived from preclinical studies. In particular, the murine C26-colon carcinoma (C-26) and Lewis Lung carcinoma (LLC) cells have been widely used to investigate cancer cachexia both in vitro and in vivo. For example, in cell-based studies, murine C2C12 myotubes have been exposed to the C-26 and LLC cell-derived secretome, largely to investigate mechanisms underlying protein catabolism and fiber atrophy (107-109). Also, C26 and LLC tumor-bearing mice are well-established cancer cachexia models. Upon implantation, both cancer cells evolve to highly reproducible and rapidly growing tumors, which is accompanied by the development of significant weight loss (110-112). Nevertheless, although both models have highly contributed to our current understanding of the mechanisms underlying cancer-induced cachexia, Talbert and colleagues recently showed that the C-26 and LLC models do not recapitulate the muscle wasting phenotype of human cancer-induced cachexia on a molecular level (89). This lack of association between experimental and clinical data likely relates to the heterogeneous presentation of human cancers and individuals. Indeed, one monoclonal tumor cannot reflect the heterogeneity of human cancers, and not all tumors induce a systemic response to the same extent. This remains a major limitation in cancer cachexia research and highlights the need for new preclinical models that better represent cachexia features that are also present in patients and that can reproduce the heterogeneity of human cancers.

In the past decade, new technological advances in cell culture have resulted in the development of 'organoids', which are defined as 3D structures grown from stem cells that consist of organ-specific cell types which self-organize into organ-like structures following the same basic intrinsic patterning events as the organ itself (113). The resulting organoids preserve key structural and functional properties of organs and therefore provide a unique system to model human organ development and various human pathologies 'in a dish'.

The development of the organoid culture system originates from several advancements in the understanding of the intestinal stem cell niche. In 2007, Barker et al. identified the leucine-rich repeat containing G-protein-coupled receptor 5 (Lgr5) as an intestinal stem cell-specific marker gene, allowing the characterization and purification of intestinal stem cells (114). The understanding that adult intestinal stem cells can both be proliferative and long lived in vivo resulted in the use of isolated Lgr5+ stem cells as a putative cellular source for organoid cultures (114). To establish long-term organoid 
cultures, previously defined insights into the growth requirements of intestinal epithelium were carefully evaluated. Following the discovery of 1) Wnt signaling as a key pathway for stem cell maintenance in vivo $(115,116), 2)$ the Wnt agonist R-spondin (117) as a mitogen causing stem cell hyperplasia (118), and 3 ) the contrasting roles of tyrosine kinase receptor signaling, with EGF being another potent mitogen and the bone morphogenetic protein inhibitor Noggin being crucial for the maintenance of the stem cell niche (119), major components of the organoid culture medium include the niche factors R-spondin, Noggin, and EGF. Furthermore, the culture medium was supplemented with Rho kinase (ROCK) inhibitor to inhibit anoikis of primary cultures, a phenomenon which has previously been observed in purified colonic epithelial cells (120). Anoikis is a form of regulated cell death that occurs when anchorage-dependent cells detach from the surrounding basement membrane. The basement membrane, a form of extracellular matrix (ECM), provides structural support to epithelial cells and forms a mechanical connection between the epithelia and underlying connective tissue. Previous studies have shown that feeder layer cultures, in which fibroblasts produces ECM proteins, support stem cell growth (121). In addition, the pre-treatment of plastic tissue culture surfaces with ECM proteins, such as collagen or laminin, have been shown to further enhance stem cell clonogenicity. Furthermore, a solubilized basement membrane preparation extracted from the Engelbregh-Hilm-Swarm (EHS) mouse sarcoma, which is a tumor rich in the ECM proteins laminin, collagen IV, heparin sulfate, proteoglycans, and entactin/nidogen, has been shown to promote 3D aggregations and polarization of (stem cells) (122). Given these essential properties of ECM proteins, purified Lgr5+ stem cells were embedded in Matrigel, which is an ex vivo substitute for the ECM. In 2009, Hans Clevers' lab was the first to describe the successful establishment of epithelial organoid cultures from single Lgr5+ stem cells embedded in Matrigel and provided with culture medium containing R-spondin-1, Noggin, and EGF (123). The addition of Wnt3a resulted in the generation of organoids from mouse colon crypts as well as purified Lgr5+ colonic stem cells (124). Furthermore, in addition to the niche factors Wnt3a, R-spondin-1, Noggin, and EGF, the supplementation of the vitamin nicotinamide, A83-01, a small-molecule inhibitor of transforming growth factor $\beta$ type I receptor kinase, and SB202190, a p38 mitogen-activated protein kinase (MAPK) inhibitor, was required for the establishment of long-term organoid cultures from primary adult human epithelial cells $(124,125)$. Over the past years, modification of the growth factors supplemented to the organoid culture medium have resulted in the establishment of epithelial organoid cultures from several other organs, including breast (126), gallbladder (127), gastric (128), head and neck (129), liver (130), pancreas (131), and prostate cancer (132).

In recent years, several aspects of cancer biology have been shown to be accurately modelled by organoids. Similar to organoids originating from healthy epithelial tissue, tumor organoids can be efficiently established by culturing primary epithelial tumor cells in 
basement membrane extract and a defined, tissue-specific growth medium. Furthermore, tumor organoids have been shown to closely recapitulate pathophysiologically aspects of pancreatic, colon, breast, and gastric cancer both in vitro and in vivo $(126,128,131,133)$. For example, Boj and colleagues showed that orthotopically transplanted pancreatic tumor organoids recapitulate the full spectrum of tumor development and progression (131). More importantly, tumor organoids have also been shown to maintain the genetic and phenotypic features of the tumor of origin, supporting their applicability as a pre-clinical model to study disease-specific mechanisms $(126,128,133)$.

\section{Aim and outline of the thesis}

Management of cachexia remains a significant challenge for patients and doctors in the clinic. Experimental preclinical systems that model human cancer-associated cachexia have tremendously contributed to our current understanding of this pathophysiology. However, progress on the prevention, early diagnosis, and treatment of cancer cachexia is highly dependent on the successful translation of novel mechanistic findings into clinical benefits, which still remains a major challenge. Therefore, a better understanding of the cachexiainducing mechanisms is crucial to prevent the onset and progression of cachexia and to improve overall survival of cancer patients. To gain better insight into human cancerinduced cachexia, there is an urgent need for better experimental preclinical models that closely recapitulate the pathophysiological aspects of this syndrome.

Modeling human pancreatic cancer with organoids could represent a powerful novel approach to study direct cachexia-inducing properties of pancreatic cancer cells. The high efficiency with which primary tumor organoid cultures can be established in combination with thorough phenotyping of cachexia-related parameters of donor patients may enable the application of organoids for understanding common as well as patientspecific cachexia-inducing mechanisms. As such, the overall aim of the studies described in this thesis was to generate and characterize patient-derived tumor organoids from wellphenotyped cachectic pancreatic cancer patients to provide a novel platform for studying the cachexia-inducing properties of pancreatic tumor cells and the mechanisms involved (Fig. 2).

Chapter 2 describes a systematic approach used to generate a living pancreatic tumor organoid biobank from tumor biopsies of patients of whom the cachexia status was thoroughly assessed. The established organoid cultures were characterized by means of histology, targeted mutation analysis, and expression of known cachexia-related factors. In chapter 3, we applied the established pancreatic tumor organoid cultures to investigate their cachexia-inducing potential. In this study, tumor organoids from a severely cachectic and a non-cachectic patient were used to assess the direct effect of tumor-derived factors on cultured skeletal muscle cells, focusing on pathways controlling skeletal muscle mass. 
It is currently not known whether the cancer cachexia-induced changes in skeletal and cardiac muscle are also present in smooth muscle, despite the important role of smooth muscle for the normal contractile functionality of the Gl-tract. In chapter 4, we first developed a novel visceral SMC culture system in which SMCs can be kept in either a highly contractile state or modulated toward the synthetic phenotype in a controlled way. This in vitro model was used in chapter 5 to investigate the direct effects of the tumor organoid secretome of cachectic and non-cachectic patients on the SMC phenotype. In chapter 6, we explored characteristics of the intestinal smooth musculature in a cohort of pancreatic cancer patients with and without a low skeletal muscle mass. Finally, in chapter 7, we summarize and discuss the data presented in this thesis and reflect to the future perspectives. 


\section{Modeling cachexia-associated muscle alterations with patient-derived pancreatic tumor organoids}

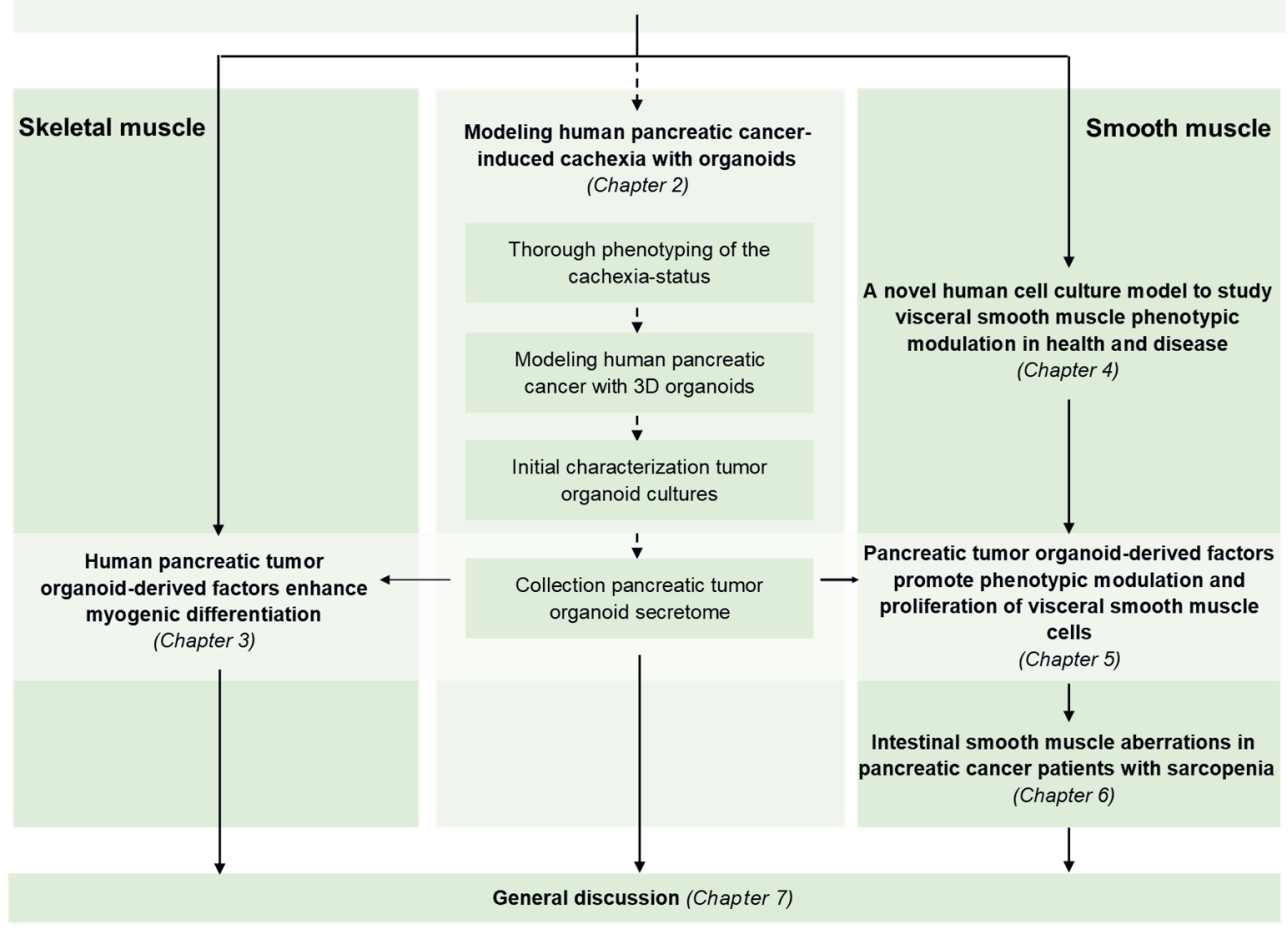

Figure 2: Thesis outline 


\section{References}

1. Kleeff J, Korc M, Apte M, La Vecchia C, Johnson CD, Biankin AV, et al. Pancreatic cancer. Nat Rev Dis Primers. 2016;2:16022.

2. Bray F, Ferlay J, Soerjomataram I, Siegel RL, Torre LA, Jemal A. Global cancer statistics 2018: GLOBOCAN estimates of incidence and mortality worldwide for 36 cancers in 185 countries. CA Cancer J Clin. 2018;68(6):394-424.

3. IKNL. Nederlandse Kankerregistratie (NKR).

4. Waters AM, Der CJ. KRAS: The Critical Driver and Therapeutic Target for Pancreatic Cancer. Cold Spring Harb Perspect Med. 2018;8(9).

5. Collisson EA, Bailey P, Chang DK, Biankin AV. Molecular subtypes of pancreatic cancer. Nat Rev Gastroenterol Hepatol. 2019;16(4):207-20.

6. Fogel EL, Shahda S, Sandrasegaran K, DeWitt J, Easler JJ, Agarwal DM, et al. A Multidisciplinary Approach to Pancreas Cancer in 2016: A Review. Am J Gastroenterol. 2017;112(4):537-54.

7. van Rijssen LB, Rombouts SJ, Walma MS, Vogel JA, Tol JA, Molenaar IQ, et al. Recent Advances in Pancreatic Cancer Surgery of Relevance to the Practicing Pathologist. Surg Pathol Clin. 2016;9(4):539-45.

8. Conroy T, Desseigne F, Ychou M, Bouche O, Guimbaud R, Becouarn Y, et al. FOLFIRINOX versus gemcitabine for metastatic pancreatic cancer. N Eng/ J Med. 2011;364(19):1817-25.

9. Ozola Zalite I, Zykus R, Francisco Gonzalez M, Saygili F, Pukitis A, Gaujoux S, et al. Influence of cachexia and sarcopenia on survival in pancreatic ductal adenocarcinoma: a systematic review. Pancreatology. 2015;15(1):19-24.

10. Vigano A, Bruera E, Jhangri GS, Newman SC, Fields AL, Suarez-Almazor ME. Clinical survival predictors in patients with advanced cancer. Arch Intern Med. 2000;160(6):861-8.

11. Bachmann J, Heiligensetzer M, Krakowski-Roosen H, Buchler MW, Friess H, Martignoni ME. Cachexia worsens prognosis in patients with resectable pancreatic cancer. J Gastrointest Surg. 2008;12(7):1193-201.

12. Maddocks M, Byrne A, Johnson CD, Wilson RH, Fearon KC, Wilcock A. Physical activity level as an outcome measure for use in cancer cachexia trials: a feasibility study. Support Care Cancer. 2010;18(12):1539-44.

13. Andreyev HJ, Norman AR, Oates J, Cunningham D. Why do patients with weight loss have a worse outcome when undergoing chemotherapy for gastrointestinal malignancies? Eur J Cancer. 1998;34(4):503-9.

14. Persson C, Glimelius B. The relevance of weight loss for survival and quality of life in patients with advanced gastrointestinal cancer treated with palliative chemotherapy. Anticancer Res. 2002;22(6B):3661-8.

15. Ravasco P, Monteiro-Grillo I, Vidal PM, Camilo ME. Cancer: disease and nutrition are key determinants of patients' quality of life. Support Care Cancer. 2004;12(4):246-52.

16. Sadeghi M, Keshavarz-Fathi M, Baracos V, Arends J, Mahmoudi M, Rezaei N. Cancer cachexia: Diagnosis, assessment, and treatment. Crit Rev Oncol Hematol. 2018;127:91-104.

17. Bruggeman AR, Kamal AH, LeBlanc TW, Ma JD, Baracos VE, Roeland EJ. Cancer Cachexia: Beyond Weight Loss. J Oncol Pract. 2016;12(11):1163-71.

18. Fearon K, Strasser F, Anker SD, Bosaeus I, Bruera E, Fainsinger RL, et al. Definition and classification of cancer cachexia: an international consensus. Lancet Oncol. 2011;12(5):48995.

19. McDonald JJ, Fallon MT, Laird BJA. Meaningful measures in cancer cachexia: implications for practice and research. Curr Opin Support Palliat Care. 2019;13(4):323-7. 
20. Miller J, Wells L, Nwulu U, Currow D, Johnson MJ, Skipworth RJE. Validated screening tools for the assessment of cachexia, sarcopenia, and malnutrition: a systematic review. Am J Clin Nutr. 2018;108(6):1196-208.

21. Marshall S, Agarwal E. Comparing Characteristics of Malnutrition, Starvation, Sarcopenia, and achexia in Older Adults: Preedy V., Patel V. (eds) Handbook of Famine, Starvation, and Nutrient Deprivation. Springer, Cham; 2019.

22. Martin L, Birdsell L, Macdonald N, Reiman T, Clandinin MT, McCargar L, et al. Cancer cachexia in the age of obesity: skeletal muscle depletion is a powerful prognostic factor, independent of body mass index. J Clin Oncol. 2013;31(12):1539-47.

23. Arends J, Bachmann P, Baracos V, Barthelemy N, Bertz H, Bozzetti F, et al. ESPEN guidelines on nutrition in cancer patients. Clin Nutr. 2017;36(1):11-48.

24. Hebuterne X, Lemarie E, Michallet M, de Montreuil CB, Schneider SM, Goldwasser F. Prevalence of malnutrition and current use of nutrition support in patients with cancer. JPEN J Parenter Enteral Nutr. 2014;38(2):196-204.

25. Zhang X, Edwards BJ. Malnutrition in Older Adults with Cancer. Curr Oncol Rep. 2019;21(9):80.

26. Detsky AS, Baker JP, O'Rourke K, Johnston N, Whitwell J, Mendelson RA, et al. Predicting nutrition-associated complications for patients undergoing gastrointestinal surgery. JPEN J Parenter Enteral Nutr. 1987;11(5):440-6.

27. Ottery FD. Definition of standardized nutritional assessment and interventional pathways in oncology. Nutrition. 1996;12(1 Suppl):S15-9.

28. Blum D, Omlin A, Fearon K, Baracos V, Radbruch L, Kaasa S, et al. Evolving classification systems for cancer cachexia: ready for clinical practice? Support Care Cancer. 2010;18(3):273-9.

29. Cavalcante Martins FF, de Pinho NB, de Carvalho Padilha P, Martucci RB, Rodrigues VD, Sales $\mathrm{RC}$, et al. Patient-generated subjective global assessment predicts cachexia and death in patients with head, neck and abdominal cancer: A retrospective longitudinal study. Clin Nutr ESPEN. 2019;31:17-22.

30. Guigoz $\mathrm{Y}$, Lauque $\mathrm{S}$, Vellas BJ. Identifying the elderly at risk for malnutrition. The Mini Nutritional Assessment. Clin Geriatr Med. 2002;18(4):737-57.

31. Guigoz Y, Vellas B, Garry PJ. Assessing the nutritional status of the elderly: The Mini Nutritional Assessment as part of the geriatric evaluation. Nutr Rev. 1996;54(1 Pt 2):S59-65.

32. Cawood AL, Elia M, Sharp SK, Stratton RJ. Malnutrition self-screening by using MUST in hospital outpatients: validity, reliability, and ease of use. Am J Clin Nutr. 2012;96(5):1000-7.

33. Dev R. Measuring cachexia-diagnostic criteria. Ann Palliat Med. 2019;8(1):24-32.

34. Durnin JV, Womersley J. Body fat assessed from total body density and its estimation from skinfold thickness: measurements on 481 men and women aged from 16 to 72 years. Br J Nutr. 1974;32(1):77-97.

35. Frisancho AR. Triceps skin fold and upper arm muscle size norms for assessment of nutrition status. Am J Clin Nutr. 1974;27(10):1052-8.

36. Pichard C, Kyle UG. Body composition measurements during wasting diseases. Curr Opin Clin Nutr Metab Care. 1998;1(4):357-61.

37. Frisancho AR. New norms of upper limb fat and muscle areas for assessment of nutritional status. Am J Clin Nutr. 1981;34(11):2540-5.

38. Prado CM, Birdsell LA, Baracos VE. The emerging role of computerized tomography in assessing cancer cachexia. Curr Opin Support Palliat Care. 2009;3(4):269-75.

39. Mourtzakis M, Prado CM, Lieffers JR, Reiman T, McCargar LJ, Baracos VE. A practical and precise approach to quantification of body composition in cancer patients using computed tomography images acquired during routine care. Appl Physiol Nutr Metab. 2008;33(5):9971006. 
40. Basile D, Parnofiello A, Vitale MG, Cortiula F, Gerratana L, Fanotto V, et al. The IMPACT study: early loss of skeletal muscle mass in advanced pancreatic cancer patients. J Cachexia Sarcopenia Muscle. 2019;10(2):368-77.

41. Prado CM, Lieffers JR, McCargar L, Reiman T, Sawyer MB, Martin L, et al. Prevalence and clinical implications of sarcopenic obesity in patients with solid tumours of the respiratory and gastrointestinal tracts: a population-based study. Lancet Oncol. 2008;9(7):629-35.

42. van Dijk DP, Bakens MJ, Coolsen MM, Rensen SS, van Dam RM, Bours MJ, et al. Low skeletal muscle radiation attenuation and visceral adiposity are associated with overall survival and surgical site infections in patients with pancreatic cancer. J Cachexia Sarcopenia Muscle. 2017;8(2):317-26.

43. de Paula NS, Rodrigues CS, Chaves GV. Comparison of the prognostic value of different skeletal muscle radiodensity parameters in endometrial cancer. Eur J Clin Nutr. 2019;73(4):524-30.

44. Rollins KE, Tewari N, Ackner A, Awwad A, Madhusudan S, Macdonald IA, et al. The impact of sarcopenia and myosteatosis on outcomes of unresectable pancreatic cancer or distal cholangiocarcinoma. Clin Nutr. 2016;35(5):1103-9.

45. Oken MM, Creech RH, Tormey DC, Horton J, Davis TE, McFadden ET, et al. Toxicity and response criteria of the Eastern Cooperative Oncology Group. Am J Clin Oncol. 1982;5(6):649-55.

46. Kilgour RD, Vigano A, Trutschnigg B, Lucar E, Borod M, Morais JA. Handgrip strength predicts survival and is associated with markers of clinical and functional outcomes in advanced cancer patients. Support Care Cancer. 2013;21(12):3261-70.

47. Prado CM, Lieffers JR, Bowthorpe L, Baracos VE, Mourtzakis M, McCargar LJ. Sarcopenia and physical function in overweight patients with advanced cancer. Can J Diet Pract Res. 2013;74(2):69-74.

48. Argiles JM, Stemmler B, Lopez-Soriano FJ, Busquets S. Inter-tissue communication in cancer cachexia. Nat Rev Endocrinol. 2018;15(1):9-20.

49. McMillan DC. Systemic inflammation, nutritional status and survival in patients with cancer. Curr Opin Clin Nutr Metab Care. 2009;12(3):223-6.

50. Moses AG, Maingay J, Sangster K, Fearon KC, Ross JA. Pro-inflammatory cytokine release by peripheral blood mononuclear cells from patients with advanced pancreatic cancer: relationship to acute phase response and survival. Oncol Rep. 2009;21(4):1091-5.

51. Stephens NA, Skipworth RJ, Fearon KC. Cachexia, survival and the acute phase response. Curr Opin Support Palliat Care. 2008;2(4):267-74.

52. Douglas E, McMillan DC. Towards a simple objective framework for the investigation and treatment of cancer cachexia: the Glasgow Prognostic Score. Cancer Treat Rev. 2014;40(6):685-91.

53. Giannousi Z, Gioulbasanis I, Pallis AG, Xyrafas A, Dalliani D, Kalbakis K, et al. Nutritional status, acute phase response and depression in metastatic lung cancer patients: correlations and association prognosis. Support Care Cancer. 2012;20(8):1823-9.

54. Gioulbasanis I, Pallis A, Vlachostergios PJ, Xyrafas A, Giannousi Z, Perdikouri IE, et al. The Glasgow Prognostic Score (GPS) predicts toxicity and efficacy in platinum-based treated patients with metastatic lung cancer. Lung Cancer. 2012;77(2):383-8.

55. Barker T, Fulde G, Moulton B, Nadauld LD, Rhodes T. An elevated neutrophil-to-lymphocyte ratio associates with weight loss and cachexia in cancer. Sci Rep. 2020;10(1):7535.

56. Derman BA, Macklis JN, Azeem MS, Sayidine S, Basu S, Batus M, et al. Relationships between longitudinal neutrophil to lymphocyte ratios, body weight changes, and overall survival in patients with non-small cell lung cancer. BMC Cancer. 2017;17(1):141.

57. Baracos VE, Martin L, Korc M, Guttridge DC, Fearon KCH. Cancer-associated cachexia. Nat Rev Dis Primers. 2018;4:17105. 
58. Acharyya S, Ladner KJ, Nelsen LL, Damrauer J, Reiser PJ, Swoap S, et al. Cancer cachexia is regulated by selective targeting of skeletal muscle gene products. J Clin Invest. 2004;114(3):370-8.

59. Bonetto A, Aydogdu T, Jin X, Zhang Z, Zhan R, Puzis L, et al. JAK/STAT3 pathway inhibition blocks skeletal muscle wasting downstream of IL- 6 and in experimental cancer cachexia. $A m$ J Physiol Endocrinol Metab. 2012;303(3):E410-21.

60. Li W, Moylan JS, Chambers MA, Smith J, Reid MB. Interleukin-1 stimulates catabolism in C2C12 myotubes. Am J Physiol Cell Physiol. 2009;297(3):C706-14.

61. Tisdale MJ. Mechanisms of cancer cachexia. Physiol Rev. 2009;89(2):381-410.

62. Lee LA, Karabina A, Broadwell $L$, Leinwand LA. The ancient sarcomeric myosins found in specialized muscles. Skelet Muscle. 2019;9(1):7.

63. Schiaffino S, Dyar KA, Ciciliot S, Blaauw B, Sandri M. Mechanisms regulating skeletal muscle growth and atrophy. FEBS J. 2013;280(17):4294-314.

64. Schiaffino S, Mammucari C. Regulation of skeletal muscle growth by the IGF1-Akt/PKB pathway: insights from genetic models. Skelet Muscle. 2011;1(1):4.

65. Laplante M, Sabatini DM. mTOR signaling in growth control and disease. Cell. 2012;149(2):274-93.

66. Ma XM, Blenis J. Molecular mechanisms of mTOR-mediated translational control. Nat Rev Mol Cell Biol. 2009;10(5):307-18.

67. Bodell PW, Kodesh E, Haddad F, Zaldivar FP, Cooper DM, Adams GR. Skeletal muscle growth in young rats is inhibited by chronic exposure to IL- 6 but preserved by concurrent voluntary endurance exercise. J Appl Physiol (1985). 2009;106(2):443-53.

68. Emery PW, Lovell L, Rennie MJ. Protein synthesis measured in vivo in muscle and liver of cachectic tumor-bearing mice. Cancer Res. 1984;44(7):2779-84.

69. Smith KL, Tisdale MJ. Increased protein degradation and decreased protein synthesis in skeletal muscle during cancer cachexia. Br J Cancer. 1993;67(4):680-5.

70. White JP, Baynes JW, Welle SL, Kostek MC, Matesic LE, Sato S, et al. The regulation of skeletal muscle protein turnover during the progression of cancer cachexia in the Apc(Min/+) mouse. PLoS One. 2011;6(9):e24650.

71. White JP, Puppa MJ, Gao S, Sato S, Welle SL, Carson JA. Muscle mTORC1 suppression by IL6 during cancer cachexia: a role for AMPK. Am J Physiol Endocrinol Metab. 2013;304(10):E1042-52.

72. Emery PW, Edwards RH, Rennie MJ, Souhami RL, Halliday D. Protein synthesis in muscle measured in vivo in cachectic patients with cancer. $\mathrm{Br}$ Med $J$ (Clin Res $E d$ ). 1984;289(6445):584-6.

73. MacDonald AJ, Johns N, Stephens N, Greig C, Ross JA, Small AC, et al. Habitual Myofibrillar Protein Synthesis Is Normal in Patients with Upper GI Cancer Cachexia. Clin Cancer Res. 2015;21(7):1734-40.

74. Baracos VE. Regulation of skeletal-muscle-protein turnover in cancer-associated cachexia. Nutrition. 2000;16(10):1015-8.

75. Busquets S, Carbo N, Almendro V, Figueras M, Lopez-Soriano FJ, Argiles JM. Hyperlipemia: a role in regulating UCP3 gene expression in skeletal muscle during cancer cachexia? FEBS Lett. 2001;505(2):255-8.

76. Schiaffino S, Reggiani C. Fiber types in mammalian skeletal muscles. Physiol Rev. 2011;91(4):1447-531.

77. Lecker SH, Solomon V, Mitch WE, Goldberg AL. Muscle protein breakdown and the critical role of the ubiquitin-proteasome pathway in normal and disease states. J Nutr. 1999;129(1S Suppl):227S-37S.

78. Bodine SC, Latres E, Baumhueter S, Lai VK, Nunez L, Clarke BA, et al. Identification of ubiquitin ligases required for skeletal muscle atrophy. Science. 2001;294(5547):1704-8. 
79. Gomes MD, Lecker SH, Jagoe RT, Navon A, Goldberg AL. Atrogin-1, a muscle-specific F-box protein highly expressed during muscle atrophy. Proc Natl Acad Sci U $S$ A. 2001;98(25):14440-5.

80. Clarke BA, Drujan D, Willis MS, Murphy LO, Corpina RA, Burova E, et al. The E3 Ligase MuRF1 degrades myosin heavy chain protein in dexamethasone-treated skeletal muscle. Cell Metab. 2007;6(5):376-85.

81. Cohen S, Brault JJ, Gygi SP, Glass DJ, Valenzuela DM, Gartner C, et al. During muscle atrophy, thick, but not thin, filament components are degraded by MuRF1-dependent ubiquitylation. J Cell Biol. 2009;185(6):1083-95.

82. Sandri M, Sandri C, Gilbert A, Skurk C, Calabria E, Picard A, et al. Foxo transcription factors induce the atrophy-related ubiquitin ligase atrogin-1 and cause skeletal muscle atrophy. Cell. 2004;117(3):399-412.

83. Stitt TN, Drujan D, Clarke BA, Panaro F, Timofeyva Y, Kline WO, et al. The IGF-1/PI3K/Akt pathway prevents expression of muscle atrophy-induced ubiquitin ligases by inhibiting FOXO transcription factors. Mol Cell. 2004;14(3):395-403.

84. Cai D, Frantz JD, Tawa NE, Jr., Melendez PA, Oh BC, Lidov HG, et al. IKKbeta/NF-kappaB activation causes severe muscle wasting in mice. Cell. 2004;119(2):285-98.

85. Ma JF, Sanchez BJ, Hall DT, Tremblay AK, Di Marco S, Gallouzi IE. STAT3 promotes IFNgamma/TNFalpha-induced muscle wasting in an NF-kappaB-dependent and IL-6independent manner. EMBO Mol Med. 2017;9(5):622-37.

86. Marchildon F, Lamarche E, Lala-Tabbert N, St-Louis C, Wiper-Bergeron N. Expression of CCAAT/Enhancer Binding Protein Beta in Muscle Satellite Cells Inhibits Myogenesis in Cancer Cachexia. PLoS One. 2015;10(12):e0145583.

87. Silva KA, Dong J, Dong Y, Dong Y, Schor N, Tweardy DJ, et al. Inhibition of Stat3 activation suppresses caspase- 3 and the ubiquitin-proteasome system, leading to preservation of muscle mass in cancer cachexia. J Biol Chem. 2015;290(17):11177-87.

88. Zimmers TA, Fishel ML, Bonetto A. STAT3 in the systemic inflammation of cancer cachexia. Semin Cell Dev Biol. 2016;54:28-41.

89. Talbert EE, Cuitino MC, Ladner KJ, Rajasekerea PV, Siebert M, Shakya R, et al. Modeling Human Cancer-induced Cachexia. Cell Rep. 2019;28(6):1612-22 e4.

90. Gallagher IJ, Stephens NA, MacDonald AJ, Skipworth RJ, Husi H, Greig CA, et al. Suppression of skeletal muscle turnover in cancer cachexia: evidence from the transcriptome in sequential human muscle biopsies. Clin Cancer Res. 2012;18(10):2817-27.

91. Jagoe RT, Redfern CP, Roberts RG, Gibson GJ, Goodship TH. Skeletal muscle mRNA levels for cathepsin $\mathrm{B}$, but not components of the ubiquitin-proteasome pathway, are increased in patients with lung cancer referred for thoracotomy. Clin Sci (Lond). 2002;102(3):353-61.

92. Op den Kamp CM, Langen RC, Minnaard R, Kelders MC, Snepvangers FJ, Hesselink MK, et al. Pre-cachexia in patients with stages I-III non-small cell lung cancer: systemic inflammation and functional impairment without activation of skeletal muscle ubiquitin proteasome system. Lung Cancer. 2012;76(1):112-7.

93. Stephens NA, Gallagher IJ, Rooyackers O, Skipworth RJ, Tan BH, Marstrand T, et al. Using transcriptomics to identify and validate novel biomarkers of human skeletal muscle cancer cachexia. Genome Med. 2010;2(1):1.

94. Barkhudaryan A, Scherbakov N, Springer J, Doehner W. Cardiac muscle wasting in individuals with cancer cachexia. ESC Heart Fail. 2017;4(4):458-67.

95. Springer J, Tschirner A, Haghikia A, von Haehling S, Lal H, Grzesiak A, et al. Prevention of liver cancer cachexia-induced cardiac wasting and heart failure. Eur Heart J. 2014;35(14):932-41.

96. Dudgeon D, Baracos VE. Physiological and functional failure in chronic obstructive pulmonary disease, congestive heart failure and cancer: a debilitating intersection of sarcopenia, cachexia and breathlessness. Curr Opin Support Palliat Care. 2016;10(3):236-41. 
97. Tian M, Nishijima Y, Asp ML, Stout MB, Reiser PJ, Belury MA. Cardiac alterations in cancerinduced cachexia in mice. Int J Oncol. 2010;37(2):347-53.

98. Tian M, Asp ML, Nishijima Y, Belury MA. Evidence for cardiac atrophic remodeling in cancerinduced cachexia in mice. Int J Oncol. 2011;39(5):1321-6.

99. Wysong A, Couch M, Shadfar S, Li L, Rodriguez JE, Asher S, et al. NF-kappaB inhibition protects against tumor-induced cardiac atrophy in vivo. Am J Pathol. 2011;178(3):1059-68.

100. Kazemi-Bajestani SMR, Becher H, Butts C, Basappa NS, Smylie M, Joy AA, et al. Rapid atrophy of cardiac left ventricular mass in patients with non-small cell carcinoma of the lung. $J$ Cachexia Sarcopenia Muscle. 2019;10(5):1070-82.

101. Rensen SS, Doevendans PA, van Eys GJ. Regulation and characteristics of vascular smooth muscle cell phenotypic diversity. Neth Heart J. 2007;15(3):100-8.

102. Bdolah Y, Segal A, Tanksale P, Karumanchi SA, Lecker SH. Atrophy-related ubiquitin ligases atrogin-1 and MuRF-1 are associated with uterine smooth muscle involution in the postpartum period. Am J Physiol Regul Integr Comp Physiol. 2007;292(2):R971-6.

103. Riemer RK, Heymann MA. Regulation of uterine smooth muscle function during gestation. Pediatr Res. 1998;44(5):615-27.

104. Ohama T, Hori M, Sato K, Ozaki H, Karaki H. Chronic treatment with interleukin-1beta attenuates contractions by decreasing the activities of CPI-17 and MYPT-1 in intestinal smooth muscle. J Biol Chem. 2003;278(49):48794-804.

105. Stanzel RD, Lourenssen S, Nair DG, Blennerhassett MG. Mitogenic factors promoting intestinal smooth muscle cell proliferation. Am J Physiol Cell Physiol. 2010;299(4):C805-17.

106. Zhou T, Yang K, Thapa S, Liu H, Wang B, Yu S. Differences in Symptom Burden Among Cancer Patients With Different Stages of Cachexia. J Pain Symptom Manage. 2017;53(5):919-26.

107. Jackman RW, Floro J, Yoshimine R, Zitin B, Eiampikul M, El-Jack K, et al. Continuous Release of Tumor-Derived Factors Improves the Modeling of Cachexia in Muscle Cell Culture. Front Physiol. 2017;8:738.

108. Penna F, Busquets S, Argiles JM. Experimental cancer cachexia: Evolving strategies for getting closer to the human scenario. Semin Cell Dev Biol. 2016;54:20-7.

109. Seto DN, Kandarian SC, Jackman RW. A Key Role for Leukemia Inhibitory Factor in C26 Cancer Cachexia. J Biol Chem. 2015;290(32):19976-86.

110. Bonetto A, Rupert JE, Barreto R, Zimmers TA. The Colon-26 Carcinoma Tumor-bearing Mouse as a Model for the Study of Cancer Cachexia. J Vis Exp. 2016(117).

111. Brown JL, Lee DE, Rosa-Caldwell ME, Brown LA, Perry RA, Haynie WS, et al. Protein imbalance in the development of skeletal muscle wasting in tumour-bearing mice. $J$ Cachexia Sarcopenia Muscle. 2018;9(5):987-1002.

112. Suzuki T, Von Haehling S, Springer J. Promising models for cancer-induced cachexia drug discovery. Expert Opin Drug Discov. 2020;15(5):627-37.

113. Lancaster MA, Huch M. Disease modelling in human organoids. Dis Model Mech. 2019;12(7).

114. Barker N, van Es JH, Kuipers J, Kujala P, van den Born M, Cozijnsen M, et al. Identification of stem cells in small intestine and colon by marker gene Lgr5. Nature. 2007;449(7165):10037.

115. Korinek V, Barker N, Moerer P, van Donselaar E, Huls G, Peters PJ, et al. Depletion of epithelial stem-cell compartments in the small intestine of mice lacking Tcf-4. Nat Genet. 1998;19(4):379-83.

116. Pinto D, Gregorieff A, Begthel H, Clevers H. Canonical Wnt signals are essential for homeostasis of the intestinal epithelium. Genes Dev. 2003;17(14):1709-13.

117. de Lau W, Barker N, Low TY, Koo BK, Li VS, Teunissen H, et al. Lgr5 homologues associate with Wnt receptors and mediate R-spondin signalling. Nature. 2011;476(7360):293-7.

118. Kim KA, Kakitani M, Zhao J, Oshima T, Tang T, Binnerts M, et al. Mitogenic influence of human R-spondin1 on the intestinal epithelium. Science. 2005;309(5738):1256-9. 
119. Haramis AP, Begthel H, van den Born M, van Es J, Jonkheer S, Offerhaus GJ, et al. De novo crypt formation and juvenile polyposis on BMP inhibition in mouse intestine. Science. 2004;303(5664):1684-6.

120. Hofmann C, Obermeier F, Artinger M, Hausmann M, Falk W, Schoelmerich J, et al. Cell-cell contacts prevent anoikis in primary human colonic epithelial cells. Gastroenterology. 2007;132(2):587-600.

121. Jensen KB, Driskell RR, Watt FM. Assaying proliferation and differentiation capacity of stem cells using disaggregated adult mouse epidermis. Nat Protoc. 2010;5(5):898-911.

122. Xu C, Inokuma MS, Denham J, Golds K, Kundu P, Gold JD, et al. Feeder-free growth of undifferentiated human embryonic stem cells. Nat Biotechnol. 2001;19(10):971-4.

123. Sato T, Vries RG, Snippert HJ, van de Wetering M, Barker N, Stange DE, et al. Single Lgr5 stem cells build crypt-villus structures in vitro without a mesenchymal niche. Nature. 2009;459(7244):262-5.

124. Sato T, Stange DE, Ferrante M, Vries RG, Van Es JH, Van den Brink S, et al. Long-term expansion of epithelial organoids from human colon, adenoma, adenocarcinoma, and Barrett's epithelium. Gastroenterology. 2011;141(5):1762-72.

125. Jung P, Sato T, Merlos-Suarez A, Barriga FM, Iglesias M, Rossell D, et al. Isolation and in vitro expansion of human colonic stem cells. Nat Med. 2011;17(10):1225-7.

126. Sachs N, de Ligt J, Kopper O, Gogola E, Bounova G, Weeber F, et al. A Living Biobank of Breast Cancer Organoids Captures Disease Heterogeneity. Cell. 2018;172(1-2):373-86 e10.

127. Saito Y, Muramatsu T, Kanai Y, Ojima H, Sukeda A, Hiraoka N, et al. Establishment of PatientDerived Organoids and Drug Screening for Biliary Tract Carcinoma. Cell Rep. 2019;27(4):1265-76 e4.

128. Yan HHN, Siu HC, Law S, Ho SL, Yue SSK, Tsui WY, et al. A Comprehensive Human Gastric Cancer Organoid Biobank Captures Tumor Subtype Heterogeneity and Enables Therapeutic Screening. Cell Stem Cell. 2018;23(6):882-97 e11.

129. Driehuis E, Kolders S, Spelier S, Lohmussaar K, Willems SM, Devriese LA, et al. Oral Mucosal Organoids as a Potential Platform for Personalized Cancer Therapy. Cancer Discov. 2019;9(7):852-71.

130. Broutier L, Mastrogiovanni G, Verstegen MM, Francies HE, Gavarro LM, Bradshaw CR, et al. Human primary liver cancer-derived organoid cultures for disease modeling and drug screening. Nat Med. 2017;23(12):1424-35.

131. Boj SF, Hwang Cl, Baker LA, Chio, II, Engle DD, Corbo V, et al. Organoid models of human and mouse ductal pancreatic cancer. Cell. 2015;160(1-2):324-38.

132. Gao D, Vela I, Sboner A, laquinta PJ, Karthaus WR, Gopalan A, et al. Organoid cultures derived from patients with advanced prostate cancer. Cell. 2014;159(1):176-87.

133. van de Wetering M, Francies HE, Francis JM, Bounova G, lorio F, Pronk A, et al. Prospective derivation of a living organoid biobank of colorectal cancer patients. Cell. 2015;161(4):93345. 



\section{CHAPTER 2}

\section{Generation and initial characterization of novel tumor organoid models to study human pancreatic cancer-induced cachexia}

Rianne D.W. Vaes, David P.J. van Dijk, Tessa T.J. Welbers, Marinus J. Blok, Merel R. Aberle, Lara Heij, Sylvia F. Boj, Steven W.M. Olde Damink, Sander S. Rensen Journal of Cachexia, Sarcopenia and Muscle 2020; 11:1509-1524 


\section{Abstract}

Background: The majority of patients with pancreatic cancer develop cachexia. The mechanisms underlying cancer cachexia development and progression remain elusive, though tumor-derived factors are considered to play a major role. Pancreatic tumor organoids are in vitro 3D organ-like structures that retain many pathophysiological characteristics of the in vivo tumor. We aimed to establish a pancreatic tumor organoid biobank from well-phenotyped cachectic and non-cachectic patients to enable identification of tumor-derived factors driving cancer cachexia.

Methods: Organoids were generated from tumor tissue of eight pancreatic cancer patients. A comprehensive pre-operative patient assessment of cachexia-related parameters including nutritional status, physical performance, body composition, and inflammation was performed. Tumor- and cachexia-related characteristics of the organoids were analyzed using histological stainings, targeted sequencing, and RT-qPCR. Cachexia-related factors present in the circulation of the patients and in the tumor organoid secretome were analyzed by enzyme-linked immunosorbent assay (ELISA).

Results: The established human pancreatic tumor organoids presented typical features of malignancy corresponding to the primary tumor (i.e. nuclear enlargement, multiple nucleoli, mitosis, apoptosis, and mutated KRAS and/or TP53). These tumor organoids also expressed variable levels of many known cachexia-related genes including interleukin-6 (IL6), TNF- $\alpha, I L-8, I L-1 \alpha, I L-16, M c p-1, G D F 15$, and LIF. mRNA expression of $I L-1 \alpha$ and $I L-16$ was significantly reduced in organoids from cachectic versus non-cachectic patients (IL-1 $\alpha$ : -3.8fold, $p=0.009$ and $I L-16$ : -4.7 -fold, $p=0.004)$. LIF, $I L-8$, and GDF15 mRNA expression levels were significantly higher in organoids from cachectic versus non-cachectic patients (LIF: 1.6fold, $p=0.003$; IL-8: 1.4-fold, $p=0.001 ;$ GDF15: 2.3-fold, $p<0.001)$. In line with the GDF15 and IL-8 mRNA expression levels, tumor organoids from cachectic patients secreted more GDF15 and IL-8 compared to organoids from non-cachectic patients $(5.4 \mathrm{ng} / \mathrm{ml}$ vs $1.5 \mathrm{ng} / \mathrm{ml}$, $\mathrm{p}=0.01$, and $7.4 \mathrm{ng} / \mathrm{ml}$ vs $1.3 \mathrm{ng} / \mathrm{ml}, \mathrm{p}=0.07$, respectively).

Conclusion: This novel human pancreatic tumor organoid biobank provides a valuable tool to increase our understanding of the mechanisms driving cancer cachexia. Our preliminary characterization of the secretome of these organoids supports their application in functional studies including conditioned medium approaches and in vivo transplantation models. 


\section{Introduction}

Pancreatic cancer is one of the deadliest cancer types that is responsible for approximately $4.5 \%$ of all cancer deaths worldwide (1). The high mortality rate of pancreatic cancer is related to the fact that most patients are diagnosed at advanced disease stages, when therapeutic options are limited. Late detection of pancreatic cancer is due to the absence of specific symptoms, a lack of sensitive and specific tumor markers, and difficulties in imaging early-stage tumors (2). As a result, most patients with pancreatic cancer present at the clinic because of jaundice and unexplained weight loss, the latter being indicative of the presence of a phenomenon called cancer cachexia.

Cancer cachexia is a severe wasting syndrome with multifactorial causes, involving tumor- and host tissue-derived signaling factors and alterations in metabolism that ultimately result in skeletal muscle wasting, its key phenotypic feature $(3,4)$. Cachexia is present in up to $80 \%$ of pancreatic cancer patients, and is a major contributor to their poor survival rate $(5,6)$. It is associated with reduced physical function, diminished tolerance to anticancer treatment regimens, and a marked reduction in quality of life (3). In view of the lack of effective treatment options for pancreatic cancer patients, managing cachexia is increasingly considered an attractive strategy to improve survival. However, effective pharmacologic options for the treatment of cachexia are currently lacking (7).

To develop effective and targeted anti-cachexia therapies, more insight into its underlying pathophysiological mechanisms is required. Our current understanding of the cachexia-inducing factors expressed and released by tumor cells is predominantly derived from both in vitro and in vivo studies with established murine cancer cell lines like C26 and Lewis Lung Carcinoma (8-12). Over the last five years, human pancreatic cancer cell lines such as MiaPaCa-2, Capan-1, and Panc-1 have been increasingly used to model cancerinduced cachexia (13-15) because of the high prevalence and severity of cachexia in pancreatic cancer patients. However, it is important to recognize that traditional cell culture models are comparatively artificial since cells are maintained on a stiff two-dimensional (2D) plastic surface in the absence of physiological gradients of oxygen and nutrients and without the potential for cellular-extracellular matrix interactions (16). These artificial culture conditions hence require important non-physiological cellular adaptations that are associated with mutational and chromosomal instability, which increase with prolonged culturing (17). Since most of the cell lines used in cachexia research have been cultured in 2D for decades, currently available strains are genetically different compared to the originally isolated tumor cells, potentially leading to differential activation of gene expression programs. This implies that many established pancreatic cell lines will likely have gained or lost cachexia-inducing properties, resulting in inconsistent findings among studies. On top of this, cachexia-related clinical data of the patients of whom these cells were derived are completely lacking, complicating the analysis of links between in vivo and in vitro phenotypes. Altogether, this underscores the need for better experimental models that facilitate the identification of novel cachexia-inducing targets and their ultimate translation into clinical benefit. 
Recently, several aspects of cancer biology have been shown to be accurately modelled by so-called organoid technology (18-20). Tumor organoids can be efficiently established by culturing primary epithelial tumor cells in basement membrane extract (BME) and a defined, tissue-specific growth medium. They self-organize into threedimensional (3D) structures mimicking the architecture of the organ of origin, and have been shown to closely recapitulate pathophysiologically aspects of pancreatic, colon, breast, and gastric cancer both in vitro and in vivo $(18,21-23)$. Histologic, genetic, and transcriptomic features of the original tumor have been shown to be maintained in patientderived tumor organoids, supporting their applicability as a pre-clinical model to study disease-specific mechanisms (21-23). Pancreatic tumor organoids have already proven to be useful in identifying novel genes associated with pancreatic cancer progression $(18,24)$. Moreover, emerging evidence confirms the potential of tumor organoid-based highthroughput drug screens to identify novel targeted drugs and to predict patient treatment responses $(22,25,26)$. We therefore hypothesized that modeling human pancreatic cancer with organoids could represent a powerful novel approach to study direct cachexia-inducing properties of pancreatic cancer cells. The high efficiency with which primary tumor organoid cultures can be established in combination with thorough phenotyping of cachexia-related parameters of donor patients enables the application of organoids for understanding common cachexia-inducing mechanisms as well as interindividual differences.

In this paper, we describe our systematic approach to generate a pancreatic tumor organoid biobank and the initial characterization of these organoids by means of histology, targeted mutation analysis, and analysis of expression of known cachexia-related factors. Our data reveal strong interindividual variation in the production of factors known to be involved in cachexia by tumor organoids, demonstrating its power for modeling cachexia. 


\section{Materials and Methods}

\section{Patients}

Patients undergoing pancreaticoduodenectomy at the Maastricht University Medical Centre (MUMC+) for suspected adenocarcinoma of the pancreas have been enrolled in this study. Exclusion criteria included the use of systemic glucocorticoids in the past four weeks, neoadjuvant chemo- and/or radiotherapy, and the presence of another malignancy. All patients provided written informed consent. This study was approved by the local Medical Ethics Committee (METC 13-4-107).

\section{Screening of cachexia-related parameters}

The nutritional status of the patients was thoroughly assessed by a trained physician in the outpatient clinic. The screening included measurements of body weight and height, patientreported weight loss in the last six months, upper arm circumference (27), triceps skinfold thickness (27), handgrip strength (28), patient-generated subjective global assessment (PGSGA) (29), and the malnutrition universal screening tool (MUST) (30). Systemic inflammation was assessed by measuring plasma C-reactive protein (CRP) and albumin levels preoperatively (routine in-hospital laboratory test, MUMC+). Fecal elastase levels were determined as a measure of pancreatic exocrine insufficiency. The patients provided written informed consent for retrieving the data from their medical chart.

\section{Computed tomography-based body composition}

Body composition was assessed by using computed tomography (CT) imaging and sliceOmatic 5.0 software (TomoVision, Magog, Canada) (31). Adipose tissue and skeletal muscle mass were quantified on a cross-sectional CT-image at the third lumbar (L3) vertebra that was pre-operatively acquired for diagnostic purposes. Using predefined Hounsfield Unit (HU) ranges, the total cross-sectional area $\left(\mathrm{cm}^{2}\right)$ of skeletal muscle tissue $(-29$ to 150 $\mathrm{HU})$, visceral adipose tissue (VAT) (-150 to $-50 \mathrm{HU})$, and subcutaneous tissue (SAT) (-190 to $-30 \mathrm{HU})$ was determined. The radiation attenuation for skeletal muscle was assessed by calculating the average $\mathrm{HU}$ value of the total muscle area within the specified range of -29 to $150 \mathrm{HU}$. The total areas of skeletal muscle, VAT, and SAT were normalized for stature to calculate the L3-muscle index (L3-SMI), L3-VAT index, and L3-SAT index in $\mathrm{cm}^{2} / \mathrm{m}^{2}$. Previously published sex-specific cut-off values were used for the CT-derived body composition parameters (32).

\section{Diagnosis of cancer cachexia}

Cachexia was defined according to the international consensus definition as 1) weight loss $>5 \%$ over the past 6 months in the absence of starvation, and/or 2 ) $\mathrm{BMI}<20 \mathrm{~kg} / \mathrm{m}^{2}$ and $>2 \%$ ongoing weight loss, and/or 3 ) sarcopenia and $>2 \%$ ongoing weight loss (3). Patients were diagnosed with cancer cachexia if $\geq 1$ of the criteria were met. 


\section{Collection of plasma samples and tumor biopsy}

Prior to the start of surgery, blood was collected in EDTA tubes and stored on ice until further processing. The blood was centrifuged at $1150 \times \mathrm{g}$ at $4^{\circ} \mathrm{C}$ for $12 \mathrm{~min}$ without brake. Plasma aliquots were stored at $-80^{\circ} \mathrm{C}$ until further analysis.

After removal of the pancreas specimen during surgery, the tissue was immediately transferred to the pathology laboratory (Department of Pathology, MUMC+), where a dedicated gastrointestinal pathologist identified the tumor macroscopically and collected a fresh approximately $0.5-1 \mathrm{~cm}^{3}$ tumor containing tissue slice. The tissue slice was transferred into ice-cold Advanced Dulbecco's Modified Eagle Medium/Ham's F-12 (AdvDF+++) (Gibco, Cat. No. 12634-010) supplemented with 1x GlutaMAX (Gibco, Cat. No. 35050-061), $10 \mathrm{mM}$ HEPES (Gibco, Cat. No. 15630-080), and Pen/Strep (50 units/mL penicillin and $50 \mu \mathrm{g} / \mathrm{mL}$ streptomycin) (Gibco, Cat. No 15140-122) and was stored on ice until further processing.

\section{Establishment of human pancreatic tumor organoids}

Pancreatic tumor organoids were established according to previously described protocols $(18,33)$. The tumor biopsy arrived at the Department of Surgery (Maastricht University) within 2 hours after removal of the pancreas specimen from the patient. Upon arrival, the

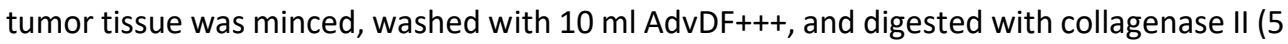
$\mathrm{mg} / \mathrm{ml}$, Gibco, Cat. No. 17101-01) in AdvDF+++ supplemented with $50 \%$ (v/v) Wnt3a conditioned medium (CM) (33) and $10 \mu \mathrm{M}$ Rho Kinase inhibitor (Y-27632) on an orbital shaker at $37^{\circ} \mathrm{C}$ for 1-2 hours. The digested tissue suspension was further digested with TrypLE (Gibco, Cat. No. 12605-010) supplemented with $10 \mu \mathrm{M}$ Rho Kinase inhibitor at $37^{\circ} \mathrm{C}$. TrypLE digestion was stopped by adding ice-cold AdvDF+++ followed by 5 min centrifugation at $350 \mathrm{xg}$ at $4^{\circ} \mathrm{C}$. Subsequently, the pellet was resuspended in ice-cold basement membrane extract (BME; Geltrex LDEV-Free Reduced Growth Factor Basement Membrane Matrix, Gibco, Cat. No. 1413202) and three $15 \mu \mathrm{L}$ droplets of Geltrex-cell suspension were allowed to solidify per well of a 24-well culture plate (Eppendorf) at $37^{\circ} \mathrm{C}$ for $30 \mathrm{~min}$. When the droplets were solidified, $500 \mu$ l of either organoid-medium ' $a$ ' or medium ' $b$ ' (Table S1, (18)) were added to each well. This resulted in the establishment of 2 organoid cultures from one individual tumor biopsy. The plate was transferred to a humidified $37^{\circ} \mathrm{C} / 5 \% \mathrm{CO}_{2}$ incubator and medium was changed every 2-3 days.

\section{Culturing of pancreatic tumor organoids}

The organoids were passaged every 7-10 days. Organoids were collected in $2 \mathrm{ml} \mathrm{AdvDF+++}$ and mechanically sheared through narrowed glass Pasteur pipettes. Following the addition of $10 \mathrm{ml} \mathrm{AdvDF+++}$ and centrifugation at $350 \mathrm{xg}\left(5 \mathrm{~min}, 4^{\circ} \mathrm{C}\right)$, organoid fragments were resuspended in ice-cold BME and plated as described above, allowing the formation of new organoids. Successfully established organoid cultures were cryopreserved after 2-5 passages. Characterization of the organoids was performed between passage number 6 and 15. 


\section{Histological characterization of tumor and tumor organoids}

Tissue and organoids were fixed in $4 \%$ paraformaldehyde followed by dehydration, paraffin embedding, sectioning, and standard haematoxylin and eosin staining. Stained organoid slides were digitalized using the Ventana iScan HT (Version 1.1, Roche, Ventana Medical Systems, Inc.) using a $200 x$ magnification. Sections were blindly analyzed by a pancreatic cancer pathologist.

\section{Mutation analysis}

Total genomic DNA was isolated from organoids using the QIAamp UCP DNA micro kit (QIAGEN). In short, organoids were collected in AdvDF+++ and centrifuged for $5 \mathrm{~min}$ at $750 \mathrm{xg}, 4^{\circ} \mathrm{C}$. The organoid pellet was further processed according to the manufacturer's instructions. Human genomic DNA was isolated from EDTA blood using the DSP DNA midi kit (QIAGEN). Targeted mutation analysis of 31 cancer related genes (Table S2) was performed using the single-molecule molecular inversion probes (smMIPs) technique (34). Sequence data was analyzed using SeqNext software from JSI. Validation of conservation of KRAS and TP53 mutations across organoids and the parent tumor was done by analyzing total genomic DNA extracted from formalin fixed paraffin embedded tumor sections using the QIAamp DNA FFPE Tissue Kit (QIAGEN) according to the manufacturer's instructions. Extracted genomic DNA was amplified by PCR using the following primers: KRAS-exon2: FW 5'-GATACACGTCTGCAGTCAACTG-3', RV 5'-GGTCCTGCACCAGTAATATGC-3'; TP53-exon5: FW 5'-GCCCTGACTTTCAACTCTGTCTC-3', RV 5'- CATCGCTATCTGAGCAGCGC-3'; TP53-exon6: FW 5'- GCGCTGCTCAGATAGCGATG-3', RV 5'- CCCAGTTGCAAACCAGACCTC-3'). Purified amplicons were sequenced by Eurofins Genomics (Germany).

\section{Quantitative real-time $P C R$}

Organoids cultured for $24 \mathrm{~h}$ in basal medium consisting of DMEM/F12 supplemented with $1 \%(\mathrm{v} / \mathrm{v})$ HEPES and $1 \%(\mathrm{v} / \mathrm{v})$ Pen/Strep were collected for mRNA expression analysis. Total RNA was extracted from organoid cultures using TRI Reagent (Sigma, St. Louis, MO) according to the manufacturer's protocol. Because the organoid samples contained a high concentration of extracellular material (BME), these lysates were centrifuged at 12,000xg for 10 minutes at $4^{\circ} \mathrm{C}$ in order to remove the insoluble material. RNA yield and quality were measured with a DeNovix DS-11 spectrophotometer. A total of $750 \mathrm{ng}$ RNA was reversed transcribed to cDNA using the SensiFast cDNA Synthesis Kit according to the manufacturer's instructions (Bioline $\mathrm{GmbH}$, Germany).

CDNA was diluted (1:20) in nuclease-free $\mathrm{H}_{2} \mathrm{O}$. Each qRT-PCR reaction contained 4.7 $\mu \mathrm{l}$ diluted cDNA, $5 \mu \mathrm{l} 2 \times$ SensiMix SYBR Hi-Rox Kit (Bioline, cat. No. QT605-05), and $0.3 \mu \mathrm{l}$ primers containing $10 \mu \mathrm{M}$ of both the forward and reverse primer. Specific primer pairs for each gene of interest were ordered from Sigma; sequences are listed in table S3. To quantify mRNA expression levels, quantitative real-time PCR (qRT-PCR) analysis was performed on a LightCycler480 system (Roche) with a three-step PCR program (10 min at $95^{\circ} \mathrm{C}$ followed by 40 cycles of $15 \mathrm{sec}$ at $95^{\circ} \mathrm{C}, 15 \mathrm{sec}$ at $60^{\circ} \mathrm{C}$, and $30 \mathrm{sec}$ at $72^{\circ} \mathrm{C}$ ) followed by melting curve analysis. Melt curves were made using gradual increases in temperature of $0.1{ }^{\circ} \mathrm{C} / \mathrm{sec}$ with 
6 acquisitions/sec within a temperature range of $60^{\circ} \mathrm{C}$ to $95^{\circ} \mathrm{C}$. Relative gene expression levels were derived from the LinRegPCR (Version 2016.1) method (35) and normalized to the geometric average of two reference genes, cyclophilin A (CYPA) and $\beta$-2-microglobulin (B2M).

\section{Secretome analysis}

Cachexia-related factors present in the circulation of patients and in the tumor organoid secretome were analyzed by enzyme-linked immunosorbent assay (ELISA). The tumor organoid secretome was obtained by replacing organoid growth medium by basal medium consisting of DMEM/F12 supplemented with $1 \%(\mathrm{v} / \mathrm{v})$ HEPES and $1 \%(\mathrm{v} / \mathrm{v})$ Pen/Strep. The medium was conditioned for $24 \mathrm{~h}$ by the pancreatic tumor organoids. After $24 \mathrm{~h}$, conditioned medium (CM) was collected and centrifuged at $350 \mathrm{xg}$ for $10 \mathrm{~min}$ at $4^{\circ} \mathrm{C}$. The supernatant was centrifuged for another $20 \mathrm{~min}$ at $2,000 \times \mathrm{xg}$ at $4^{\circ} \mathrm{C}$ and the resulting conditioned medium cleared from cellular debris was aliquoted and stored at $-80^{\circ} \mathrm{C}$.

Concentrations of human IL-6 (U-CyTech Biosciences, Cat. No. CT205A) and human GDF-15 (R\&D Systems, Cat. No. DY957) in plasma and CM were determined by ELISA according to the manufacturer's protocol. Plasma and CM concentrations of human IL-8 were determined using an in-house developed ELISA (36). Absorbance was measured at a wavelength of $450 \mathrm{~nm}$ using a Spark ${ }^{\mathrm{TM}} 10 \mathrm{M}$ multimode microplate reader (Tecan).

\section{Statistical analysis}

Patient data and outcome parameters were entered in IBM SPSS 24 for Microsoft Windows ${ }^{\circledR}$ and statistical analyses were performed using the non-parametric Mann-Whitney $U$ test to compare differences between the groups. A $p$-value of $p<0.05$ was considered statistically significant. 


\section{Results}

Phenotyping of cachexia severity in patients with pancreatic cancer

To be able to relate characteristics of pancreatic tumor organoids to the cachexia status of the patient, we designed a work flow that allowed us to systematically and routinely assess the cachexia status of the patient and to collect biological materials following a standardized procedure (Fig. 1). The nutritional status of the patient was assessed pre-operatively by a trained physician in the outpatient clinic. Basic patient characteristics and cachexia-related parameters collected from the patient's medical records are presented in table 1. A total of eight patients, three males and five females, were included with a mean age of $68.5( \pm 10.9)$ years and a mean BMI of $23.8( \pm 3.7) \mathrm{kg} / \mathrm{m}^{2}$. All patients were diagnosed with a tumor in the head of the pancreas, including pancreatic ductal adenocarcinoma (PDAC) (PANCO-5, PANCO-9, PANCO-11, PANCO-12, PANCO-17, and PANCO-22), ampullary carcinoma (PANCO-6), and cholangiocarcinoma (PANCO-3); neither received neoadjuvant chemotherapy. According to the international consensus definition, five patients (PANCO3, PANCO-5, PANCO-6, PANCO-9, and PANCO-17) were diagnosed with cancer cachexia. Although this definition is mainly based on the percentage weight loss, we observed considerable heterogeneity among additional cachexia-related parameters that are often reported in the literature. Additional insight into the nutritional status of the patient was obtained by using nutrition-related screening tools and anthropometric measurements. The PG-SGA questionnaire revealed that patients in both the no-cachexia (PANCO-11, PANCO22) and cachexia group (PANCO-3, PANCO-5, PANCO-6, and PANCO-9) scored $\geq 9$, which is, according to the guidelines, indicative of a critical need of improved symptom management and/or nutrient intervention. The PG-SGA score of patients PANCO-6 and PANCO-22 was accompanied by PG-SGA global assessment category $B$, indicating that they were moderately malnourished. The PG-SGA score of patient PANCO-3 was accompanied by category $\mathrm{C}$, indicating the patient was severely malnourished. The MUST screening tool, which is used to screen for patients at risk of malnutrition, identified patients PANCO-3, PANCO-9, and PANCO-17 at high risk of malnutrition. In line, using sex- and age-specific percentiles, patients PANCO-3 $\left(2568 \mathrm{~mm}^{2},<\mathrm{p} 5\right)$, PANCO-9 $\left(4182 \mathrm{~mm}^{2},<\mathrm{p} 5\right)$, and PANCO-17 $\left(3092 \mathrm{~mm}^{2},<\mathrm{p} 5\right)$ were found to have a low upper arm muscle area, which together with the aforementioned nutritional data, indicates that these patients had a poor nutritional status.

Whereas anthropometric measurements cannot distinguish between lean muscle mass and fat mass, CT imaging can discriminate between adipose tissue, bone, organs and muscle including the degree of fatty infiltration, making it the current gold standard for body composition evaluation (37). Remarkably, CT-based body composition analysis revealed that patients who did not lose $>5 \%$ weight over the last 6 months and were not classified as cachectic (PANCO-11, PANCO-12, and PANCO-22) were nevertheless found to have both a low L3-SMI and low L3-VAT. Conversely, in the cachectic group, only patients PANCO-3 and PANCO-9 had both a low L3-SMI and a low L3-VAT.

Furthermore, substantial heterogeneity in cachexia-related biochemical parameters was observed among both cachectic and non-cachectic patients. For example, 
altered levels of inflammatory markers (CRP $>10 \mathrm{mg} / \mathrm{L}$, albumin $<35 \mathrm{~g} / \mathrm{L}$, total protein $<60 \mathrm{~g} / \mathrm{L}$, and neutrophil to lymphocyte $(\mathrm{N} / \mathrm{L})$ ratio $>3.5$ ) associated with an inflammatory state in cancer cachexia were observed in patients PANCO-3, PANCO-6, PANCO-9, and PANCO-11. In addition, patients PANCO-5, PANCO-6, PANCO-9, and PANCO-11 presented with anemia (hemoglobin $<8.2 \mathrm{mmol} / \mathrm{L}$ ).

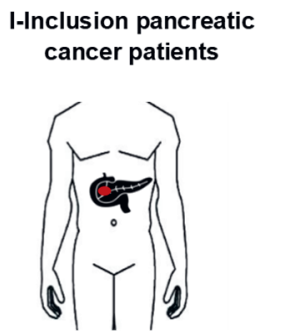

Ill-Screening cachexia-status
\begin{tabular}{ll}
\hline Short Nutritional Assessment & Height, weight, wrist circumference, upper arm \\
Procedure (SNAP) & circumference, triceps skinfold, handgrip strength \\
Malnutrition screening tools & PG-SGA, MUST \\
Blood Parameters & Inflammation, lipids, glucose \\
Computed tomography (CT) & L3-Skeletal muscle index (L3-SMI) \\
& L3-Visceral adipose tissue index (L3-VAT) \\
& L3-Subcutaneous adipose tissue index (L3-SAT) \\
& Muscle radiation attenuation (M-RA) \\
\hline
\end{tabular}

III-Establishment pancreatic tumor organoids $\begin{aligned} \text { Pancreatic tumor } & \begin{array}{l}\text { Mechanical disruption } \\ \text { \& enzymatic digestion }\end{array}\end{aligned}$

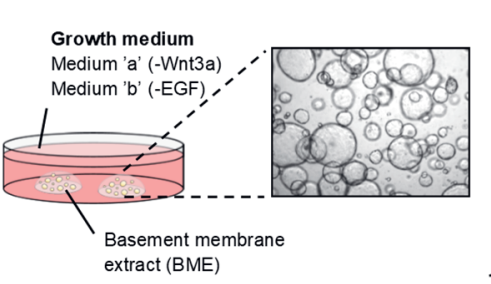

Established organoids

\begin{tabular}{ll}
\hline $\begin{array}{l}\text { Medium 'a' } \\
(\mathrm{n}=7)\end{array}$ & $\begin{array}{l}\text { Medium 'b' } \\
(\mathrm{n}=6)\end{array}$ \\
\hline PANCO-3 & PANCO-3 \\
PANCO-5 & \\
PANCO-6 & \\
PANCO-9 & PANCO-9 \\
PANCO-11 & PANCO-11 \\
PANCO-12 & PANCO-12 \\
PANCO-17 & PANCO-17 \\
& PANCO-22 \\
\hline
\end{tabular}

IV-Characterization pancreatic tumor organoids

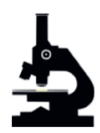

Histology

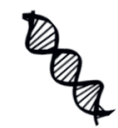

Mutation analysis

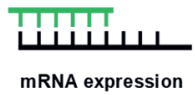

V-Expression of factors involved in cancer-induced cachexia

$\begin{array}{ll}\text { Organoid-released factors } \\ \text { IL-1 } & \text { MCP-1 } \\ \text { IL-6 } & \text { MIC-1/GDF15 } \\ \text { IL-8 } & \text { LIF } \\ \text { TNF- } \alpha & \end{array}$

Figure 1: Establishment of pancreatic tumor organoids from cachectic pancreatic cancer patients. Flowchart used to systematically and routinely assess the cachexia status of the patient and to collect biological materials following a standardized procedure. (I) Upon inclusion, (II) the nutritional status of the patients is thoroughly assessed by a trained physician in the outpatient clinic. Additional cachexia-related parameters are collected from the patient's medical records. (III) Schematic representation of the generation of human pancreatic tumor organoid culture from a tumor section from the surgically resected tumor tissue. Upon establishment, tumor cells are cultured in organoidmedium ' $a$ ' or medium ' $b$ ', resulting in the establishment of 2 organoid cultures from one individual tumor biopsy. (IV-V) Pancreatic tumor organoids are characterized by means of histology, targeted mutation analysis, and analysis of expression of known cachexia-related factors. 
Establishment of 3D tumor organoids from tumor biopsies of pancreatic cancer patients

For the establishment of tumor organoids, a section from the surgically resected tumor tissue was obtained by a pathologist. Through a combination of mechanical disruption and enzymatic digestion, pancreatic tumor cells were isolated and plated in BME droplets and overlaid with optimized pancreatic cancer organoid culture medium (Fig. 1). Considering the tumor heterogeneity and the ligand-independent activation of Ras signaling in pancreatic cancer as a consequence of the KRAS mutation in $>90 \%$ of pancreatic cancer patients (38), the organoids were placed in either a Wnt3a-depleted medium (medium ' $a$ ') or EGF-depleted medium (medium ' $b$ '). This allows selection of specific tumor clones resulting in two individual organoid cultures originating from the same tumor tissue specimen from one individual patient. Whereas we were able to establish organoid cultures from tumor tissue of all eight individual patients, from $3 / 8$ tumor specimens we could only establish pancreatic tumor organoids in either 'medium a' (without Wnt3a) or 'medium b' (without epidermal growth factor (EGF)). The resulting 13 pancreatic tumor organoid cultures were readily expanded and cryopreserved. Within this study, we further characterized these organoids by means of histology, targeted mutation analysis, and by analyzing the expression of cachexia-related factors. 


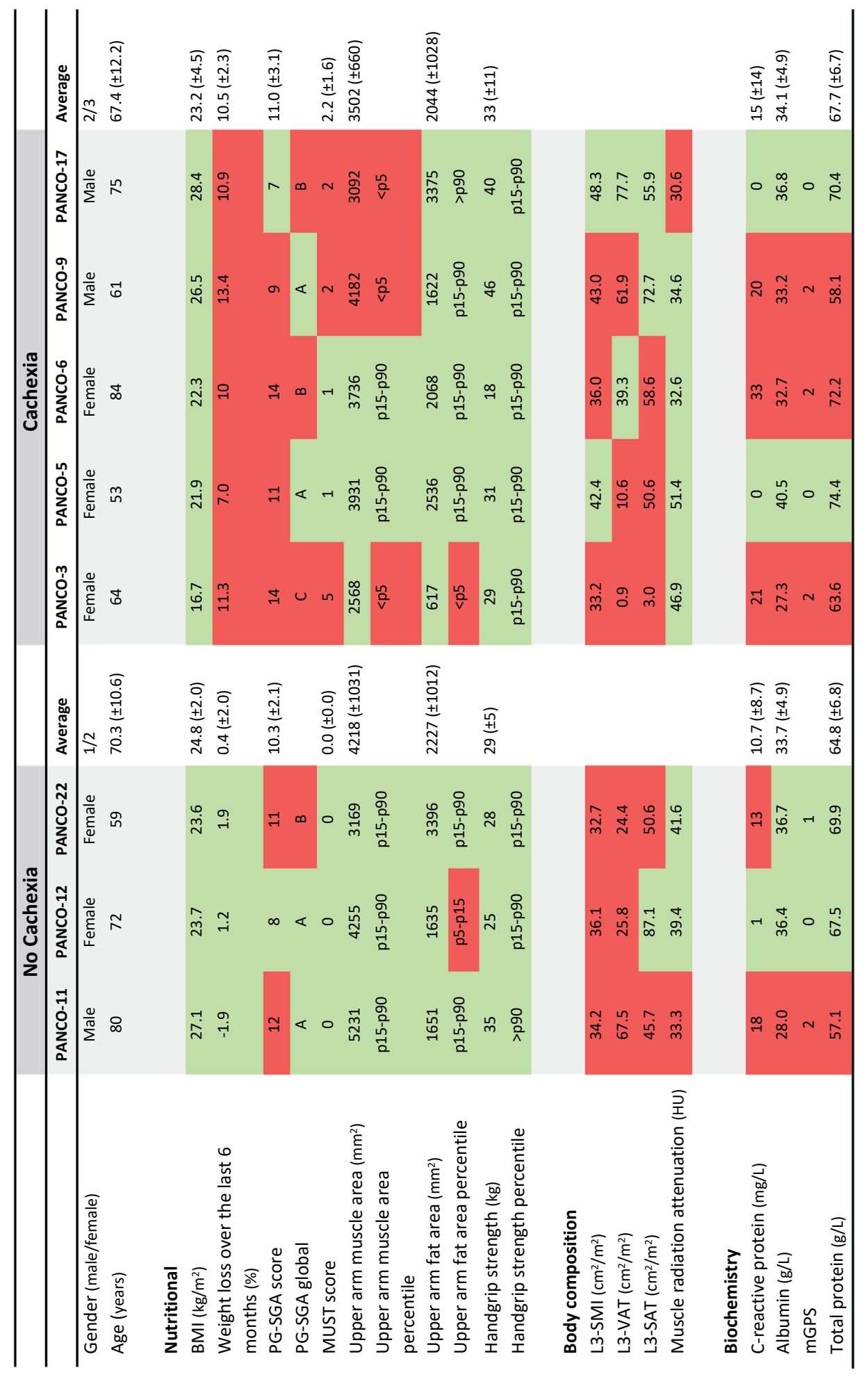




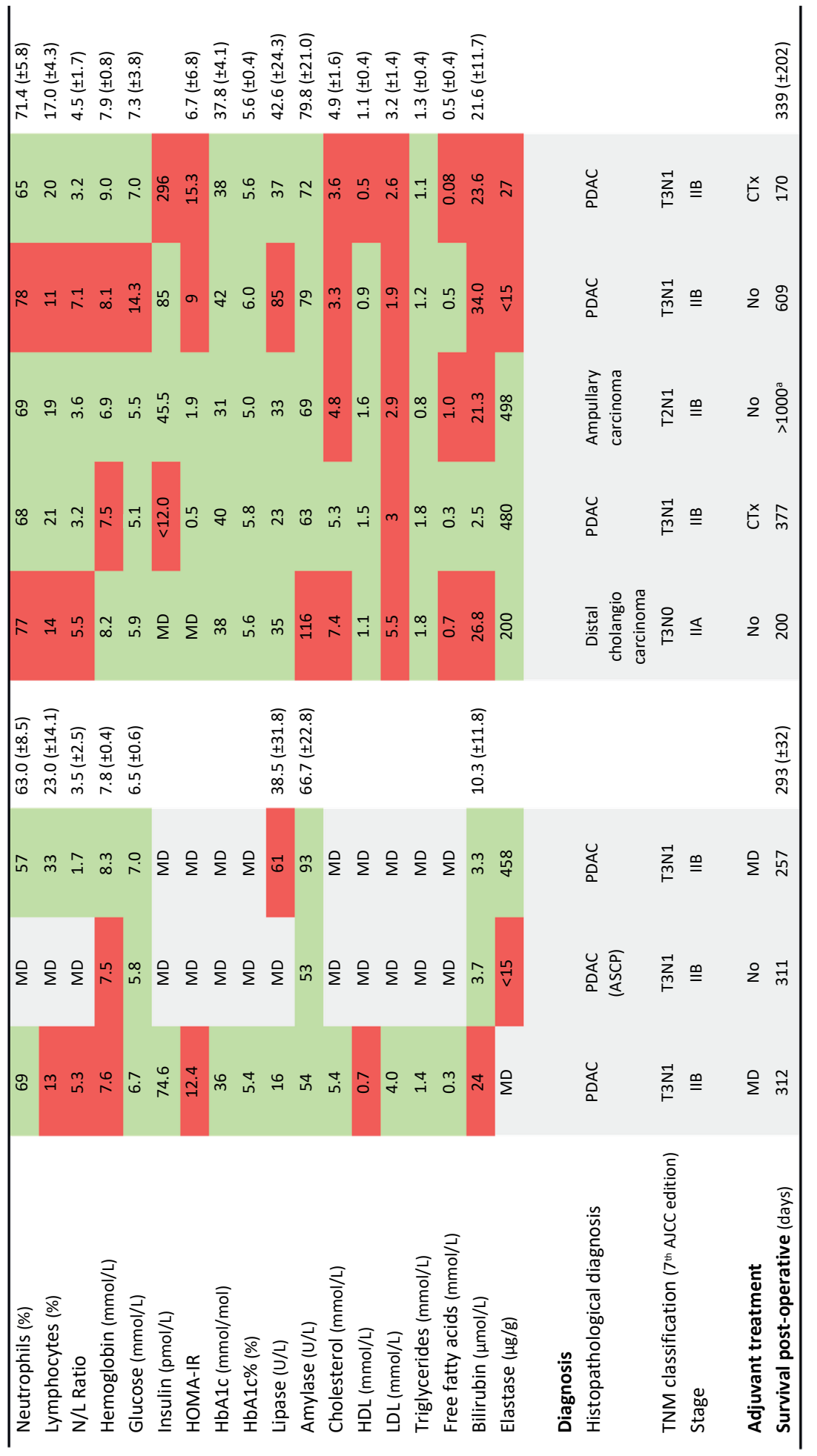




\section{Chapter 2}

$<$ Previous page. The data are presented as mean \pm standard deviation. Values within the reference range are marked in green. Values outside the reference range are marked in red. $M D=$ missing data. ASCP, adenosquamous carcinoma of the pancreas (subtype of pancreatic ductal adenocarcinoma); BMI, body mass index; CTx, chemotherapy, HDL, high density lipoprotein; HOMA-IR, Homeostatic model assessment for insulin resistance; HU, Hounsfield units; L3-SAT, L3-subcutaneous adipose tissue; L3-SMI, L3-skeletal muscle index; L3-VAT, L3-visceral adipose tissue; LDL, low density lipoprotein; mGPS, modified Glasgow prognostic score; MUST, malnutrition universal screening tool; $\mathrm{N} / \mathrm{L}$ ratio, neutrophil to lymphocyte ratio; PDAC, pancreatic ductal adenocarcinoma; PG-SGA, patientgenerated subjective global assessment. ${ }^{a}$ Post-operative survival: 1124 days and still alive at final update (09-08-2019). 


\section{Morphological characterization of pancreatic tumor organoids}

The growth and morphology of pancreatic tumor organoids varied considerably among the organoid cultures (Fig. 2A, Fig S1, and Video S1-S13). After passaging, organoids selforganized into 3D structures within $24 \mathrm{~h}$ and continued to grow to $200-400 \mu \mathrm{m}$ in diameter by day 3. Interestingly, the diameter of organoids of PANCO-12 never exceeded $200 \mu \mathrm{m}$. Furthermore, PANCO-12 organoids tended to grow in solid cell clusters whereas in general, all organoid cultures formed cohesive glandular structures with varying morphologies ranging from thin-walled cystic structures to compact organoids devoid of a lumen. No systematic differences in growth pattern were observed between the organoids cultured in either medium ' $a$ ' or medium ' $b$ '.

Next, we examined the morphology of paraffin-embedded organoids and compared them with the corresponding primary tumor specimen (Fig. 2B and Fig. S1). From a histologic point of view, the organoids showed clear similarities to their parent tumor. Pancreatic tumor organoids represented an architectural design of mostly well-formed ducts with occasionally a cribriform growth pattern (e.g. PANCO-5a) (Fig. 2B and Fig. S1). To date, pancreatic cancer is still diagnosed based on histology and graded as well differentiated, moderately differentiated, or poorly differentiated (39). Assessment of the differentiation grades of the primary tumors and their corresponding organoids revealed a similar differentiation grade, with either well-differentiated or moderately differentiated tumors or organoids (Fig. 2C). Additionally, typical nuclear features of malignancy were observed in all organoids, including nuclear enlargement, multiple nucleoli, mitoses, and apoptosis (Fig. 2D). Interestingly, we sampled an adenosquamous carcinoma of the pancreas, which is a rare variant of PDAC that represents only $1-4 \%$ of the exocrine pancreatic malignancies $(40,41)$. The organoids of this specific patient (PANCO-12) showed remarkable resemblance to the parent tumor and exhibited a mixture of glandular and squamous differentiation (Fig. 2B). Similar to the parental tumor, the organoids formed ducts with a cribriform growth pattern and cytonuclear atypia. Besides these glandular arrangements, fields of closely packed atypical cells with large abundant cytoplasm were observed. Although the squamous component of the primary tumor revealed keratinization, this component was less present in the corresponding organoid cultures (Fig. 2B and Fig. S1). 
A
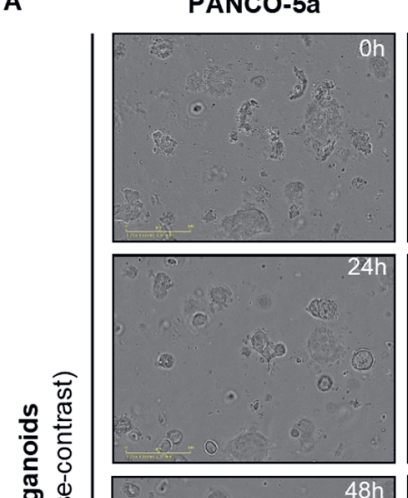

인
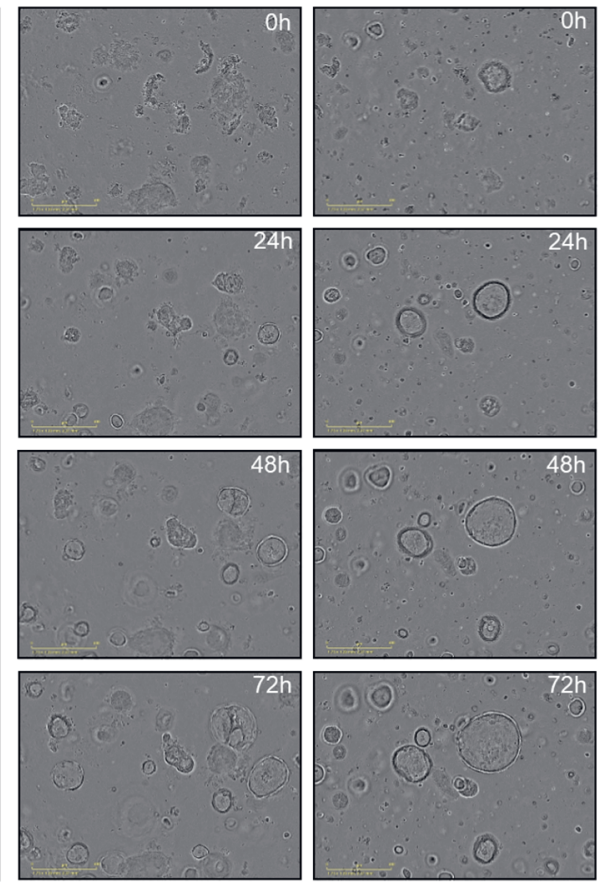

$24 \mathrm{~h}$
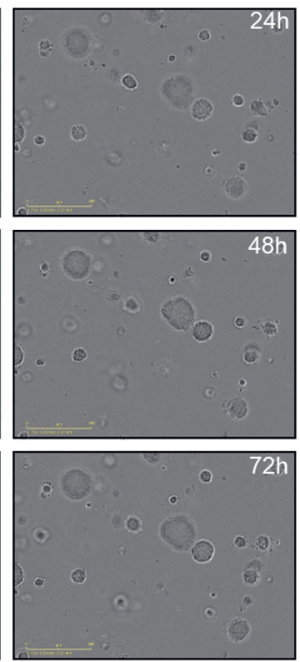

B
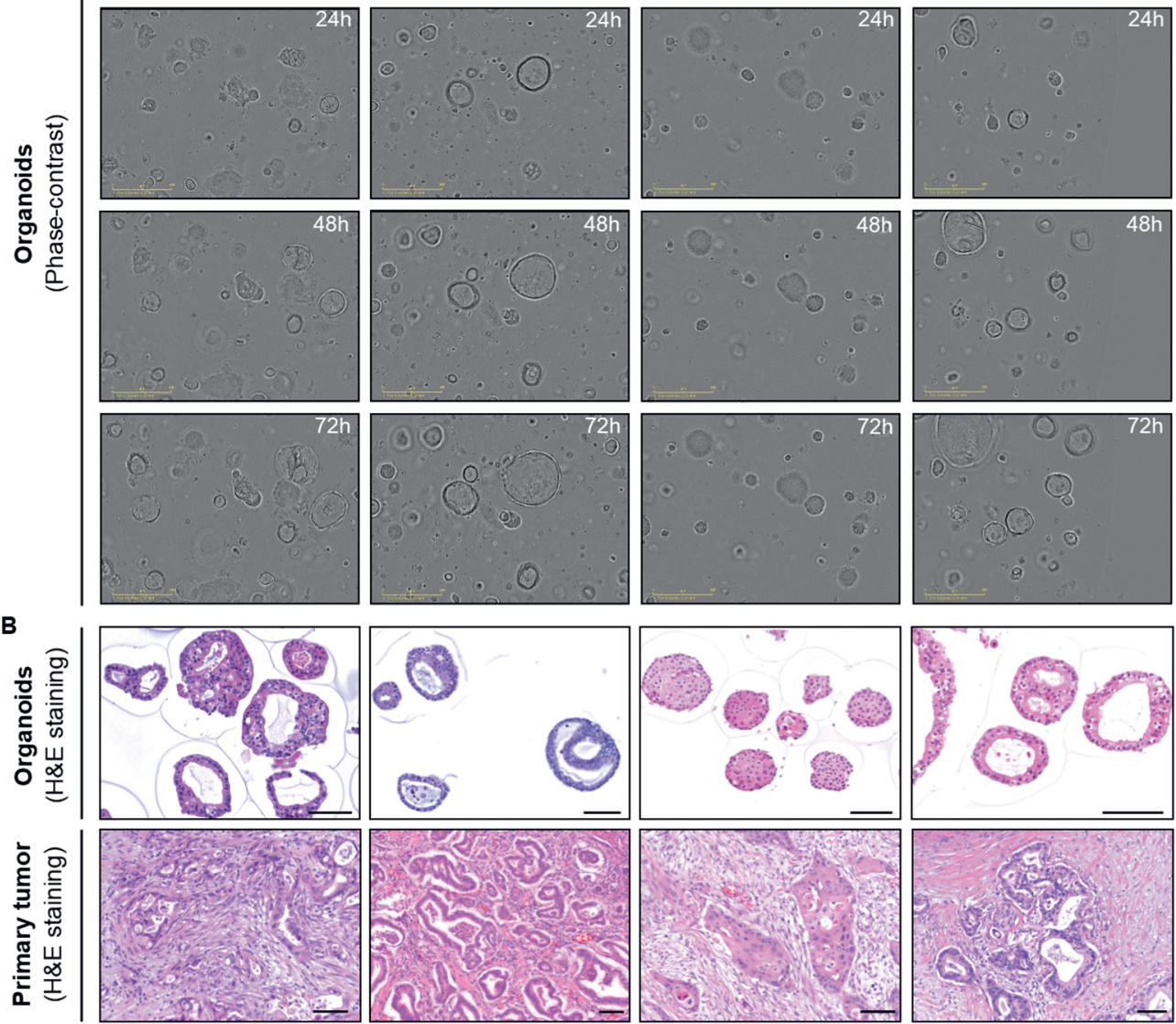

C

\begin{tabular}{lcc}
\hline & $\begin{array}{c}\text { Primary pancreatic } \\
\text { tumor }\end{array}$ & $\begin{array}{c}\text { Organoids } \\
\text { ('a' and/or 'b') }\end{array}$ \\
\hline PANCO-3 & $\mathrm{I}$ & $\mathrm{I}$ \\
PANCO-5 & $\mathrm{I}$ & $\mathrm{I}$ \\
PANCO-6 & $\mathrm{I}$ & $\mathrm{I}$ \\
PANCO-9 & $\mathrm{I}$ & $\mathrm{I}$ \\
PANCO-11 & $\mathrm{I}$ & $\mathrm{I}$ \\
PANCO-12 & $\mathrm{I}$ & $\mathrm{I}$ \\
PANCO-17 & $\mathrm{I}$ & $\mathrm{I}$ \\
PANCO-22 & $\mathrm{I}$ & $\mathrm{I}$ \\
\hline
\end{tabular}


< Previous page. Figure 2: Morphological characterization of pancreatic tumor organoids. (A) Representative phase-contrast images of pancreatic tumor organoids cultured for $72 \mathrm{~h}$ after passaging. (B) Representative hematoxylin and eosin stainings of pancreatic tumor organoids (upper panel) and the primary tumor (lower panel). Scale bar $=100 \mu \mathrm{m}$. (C) Differentiation grade of the primary tumors and their corresponding tumor organoids. Tumors are either well-differentiated (I) or moderately differentiated (II). (D) Representative hematoxylin and eosin staining of a pancreatic tumor organoid representing typical nuclear features of malignancy, including a) nuclear enlargement, b) multiple nucleoli, c) mitoses, and d) apoptosis. Scale bar $=50 \mu \mathrm{m}$.

\section{Genetic characterization of pancreatic tumor organoids}

Pancreatic cancer shows genetic homogeneity on one level with mutations in KRAS, which is detected in $>90 \%$ of pancreatic cancers. Activating KRAS point mutations at codon 12 , the most common in pancreatic cancer, result in constitutively active RAS protein (42). Targeted-sequencing of KRAS in pancreatic tumor organoids identified oncogenic KRAS mutations in all tumor-derived organoid cultures (Fig. 3 and Table S4). Whereas KRAS point mutations at codon 12 were present in all organoid cultures, the allele frequency of oncogenic KRAS variants ranged from $21.0-100 \%$, indicating differences in zygosity among the organoid cultures. Although mutations in the KRAS oncogene are typically heterozygous, organoid cultures PANCO-5a and PANCO-9b were homozygous, which has been suggested to be associated with a more aggressive tumor phenotype (43). Of note, we have established both a heterozygous (PANCO-9a) and a homozygous KRAS mutated (PANCO-9b) organoid culture originating from the same tumor section from patient PANCO-9.

Additional well-characterized gene mutations that predominate in pancreatic cancer include TP53, SMAD4, and CDKN2A of which TP53 is mutated in $>50 \%$ of the patients (44). Homozygous point mutations in the TP53 gene were identified in tumor-derived organoid cultures from 6/8 patients and were detected in the known hotspots, namely in exon 5-8 (Fig. 3 and Table S4). Analysis of KRAS and TP53 mutation status of the corresponding parent tumors confirmed the presence of the expected variants found in

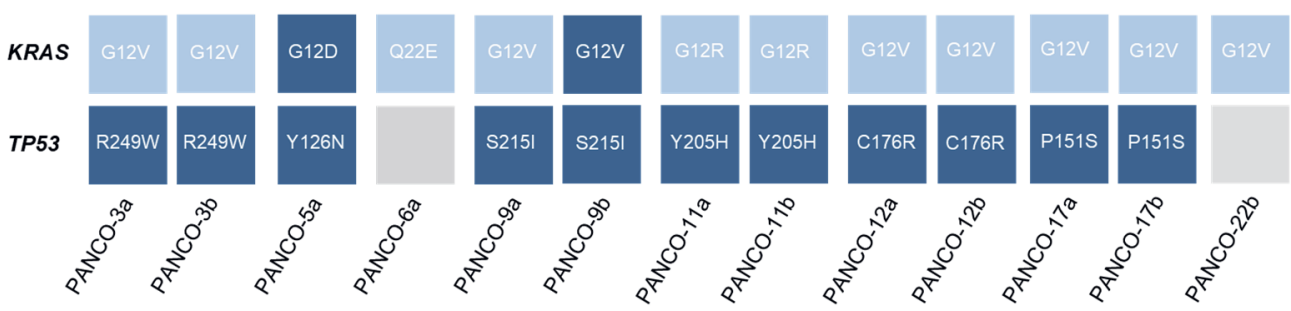

Mutation type

Wild type Heterozygous missense

Homozygous missense

Figure 3: Targeted sequencing analysis of human pancreatic tumor organoids. Targeted sequencing analysis of KRAS and TP53 including the identified substitution mutation is presented for each gene. A color legend for the type of genetic alterations is shown. 
several organoid cultures, although neoplastic cellularity in the tissue sections and/or DNA quality was not always sufficient to pick them up (Table S5, S6).

\section{Cachexia-related factors expressed by pancreatic tumor organoids}

It is thought that cancer-associated cachexia is driven by a combination of tumor-derived factors that can directly elicit catabolism in target tissues and the interplay between these tumor factors and the immune system. Together, this results in inflammation and the generation of catabolic pro-inflammatory factors (45). Tumor-derived factors previously associated with cancer cachexia mainly include pro-inflammatory cytokines such as IL-1, IL6, IL-8, TNF- $\alpha$, LIF, MIC-1/GDF15, and Mcp-1 (45). To investigate whether pancreatic tumor organoids from cachectic pancreatic cancer patients expressed higher levels of these cachexia-related factors as compared to tumor organoids from non-cachectic patients, we first assessed their gene expression levels by qPCR. Organoids were cultured for $24 \mathrm{~h}$ in basal medium to exclude potential effects of the two different medium compositions on gene expression. Pronounced differences were observed between the 'no cachexia' and 'cachexia' groups (Fig. 4A-B). Unexpectedly, significantly reduced mRNA expression levels of IL- $1 \alpha$ and IL-1 $\beta$ were detected in pancreatic tumor organoids from cachectic patients

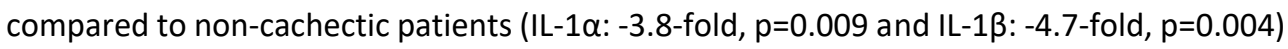
(Fig. 4B). In contrast, significantly increased mRNA expression levels of IL-8 (1.4-fold, $\mathrm{p}=0.01)$, GDF15 (2.3-fold, $\mathrm{p}<0.001)$, and LIF (1.6-fold, $\mathrm{p}=0.003$ ) were detected in tumor organoids from cachectic pancreatic cancer patients. In parallel, concentrations of secreted IL-6, IL-8, and GDF15 were measured in tumor organoid-derived CM (Table 2) and compared to the systemically circulating levels of these cytokines in the corresponding patients. Whereas IL-6 was only secreted by organoid culture PANCO-6a $(2.6 \mathrm{pg} / \mathrm{ml})$ and PANCO-9a $(5.4 \mathrm{pg} / \mathrm{ml})$, circulating levels of IL- 6 could be measured in the plasma of all non-cachectic and cachectic patients $(7.0 \pm 2.3 \mathrm{pg} / \mathrm{ml}$ vs $15.6 \pm 17.8 \mathrm{pg} / \mathrm{ml}, \mathrm{p}=0.7$, respectively). In contrast, all tumor organoids secreted relatively high levels of IL-8 and GDF15. The concentrations of these factors were significantly higher in organoids from cachectic vs non-cachectic patients (IL-8: $1.3 \pm 0.9$ vs $7.4 \pm 14.3 \mathrm{ng} / \mathrm{ml}, \mathrm{p}=0.07$ and GDF15: $1.5 \pm 1.4$ vs $5.4 \pm 3.6 \mathrm{ng} / \mathrm{ml}, \mathrm{p}=0.01$ ). 

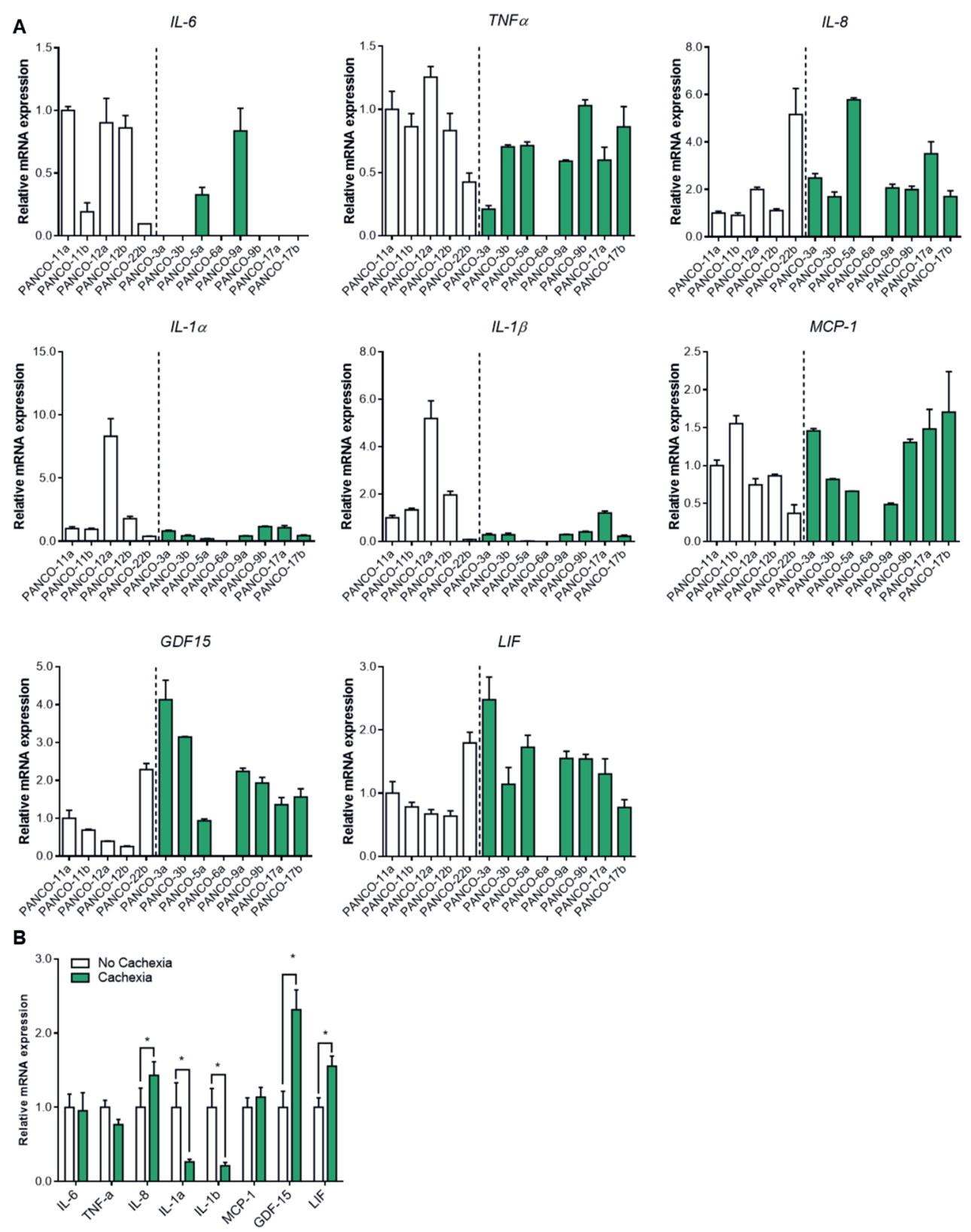

Figure 4: Levels of cachexia-related factors produced by pancreatic tumor organoids. (A) mRNA expression of cachexia-related genes was determined in human pancreatic tumor organoids from each individual patient. When 2 organoid cultures were established from one individual tumor biopsy (organoid-medium ' $a$ ' or medium ' $b$ '), the mRNA expression of both organoid cultures are shown. Data were normalized to CYPA and B2M reference genes. (B) mRNA expression of cachexia-related genes grouped by the cachexia status of the patients. Results are presented as mean \pm SE (MannWhitney $U$ test, $\left.{ }^{*} p<0.05\right)$. 


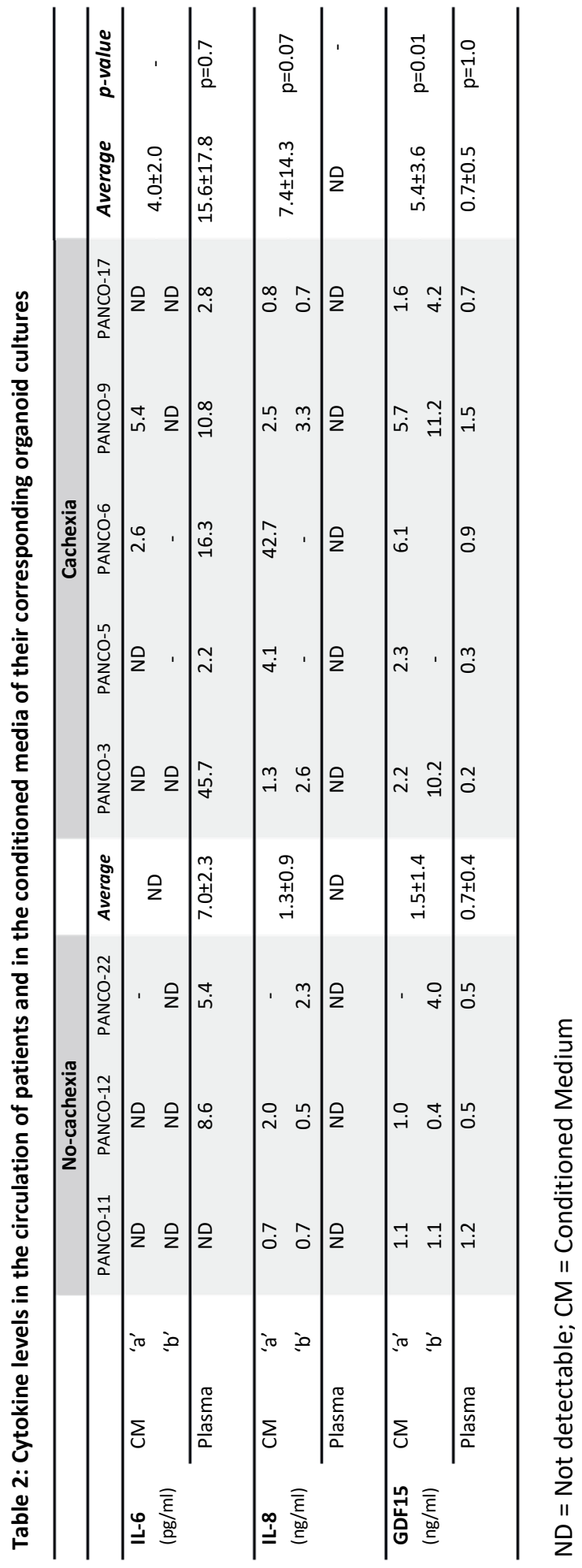




\section{Discussion}

Cancer cachexia remains a challenging problem with an important impact on quality of life and response to therapy. Despite many efforts to unravel its complex biology, the translation of novel findings into effective therapeutic targets has been hampered by a lack of experimental models that closely resemble human cancer-induced cachexia. In this study, we have generated a human pancreatic tumor organoid biobank from patients of whom the cachexia status was thoroughly assessed pre-operatively. We demonstrated that the established organoid cultures retain characteristic malignant features of the original tumor. In addition, we showed that tumor organoids express variable levels of known cachexia-associated factors including IL-6, TNF- $\alpha$, IL-8, IL-1 $\alpha$, IL-1, , Mcp-1, GDF15, and LIF. Interestingly, the interindividual variation of the production of these cachexia-associated factors was not necessarily in line with the cachexia status of the donor patient.

Several pancreatic tumor organoid libraries have been established since the development of the pancreatic tumor organoid model in 2015 (18). To show the validity of using these organoids as a representative model of the in vivo tumor, these studies have extensively characterized their morphology and transcriptional- and genetic profile in comparison with the parental tumor $(38,46,47)$. In line with these studies, the pancreatic tumor organoids established in the current study recapitulate key morphological features of the original tumor and harbor common pancreatic cancer-specific driver mutations, including KRAS and TP53. The use of these patient-derived organoid cultures to study cancer-induced cachexia has several advantages compared to currently used in vitro models. Organoids provide a better representation of the tissue of origin in comparison to monolayer cultured cells. In contrast to the stiff plastic surface that provides supraphysiological mechanical signals to cells cultured in 2D, the low stiffness and biological nature of the basement membrane extract used in organoid culture allows physiological cell-matrix protein interactions that mimic the cellular environment in tissues. This environment supports the cells to selforganize into 3D multicellular structures with apical-basal polarity that is not enforced by the culture surface as it is in traditional 2D monolayer cultures. In combination with the addition of a set of growth factors, organoid cultures are genetically stable in the long term and do not require immortalization for indefinite expansion. Before the methodology of generating organoids was developed, most 3D culture systems were spheroid based. Whereas spheroids better recapitulate in vivo tissue features than $2 \mathrm{D}$ cultures and are a simple and easy to use platform $(48,49)$, there are important differences with organoids. For example, spheroids consist of cell aggregates that develop primarily via cell-to-cell adhesion, while organoid formation is primarily driven by internal developmental processes that support the long term expansion of these structures, retaining the genetic features of the tissue of origin $(18,49,50)$. Patient-derived organoid cultures also offer several benefits over genetically engineered mouse models (GEMMs) $(51,52)$ and patient-derived xenograft models (PDXs) $(53,54)$, which are increasingly used to study the pathogenesis of cancer cachexia. First of all, organoids have been reported to retain a wide spectrum of genetic 
mutations underlying pancreatic cancer (18), in contrast to GEMMs, which usually harbor only few of the driver mutations. Secondly, organoids can be generated and applied in experimental studies in a timeframe of months, in contrast to the development of GEMMs and PDXs, which takes years. Furthermore, organoid models are much cheaper than mouse studies and have the potential to reduce and replace the use of animal models in translational research. Still, the possibility to transplant patient-derived organoids into mice offers additional opportunities to study the direct and indirect effects of tumor-derived factors involved in cancer-induced cachexia. The potential of this approach was recently demonstrated by Boj and colleagues who showed that orthotopically transplanted neoplastic organoids recapitulate the full spectrum of tumor development and progression (18). Importantly, transplanting organoids into mice allows detailed comparisons of the effects of human tumor cells on cachexia development and progression with corresponding comprehensive cachexia-related patient data. Despite these benefits of using organoids, some limitations of the organoid model should be acknowledged, including the labor intensive aspects of maintaining organoid cultures in comparison to traditional cell culture and the higher costs of culture reagents. Current organoid models also lack other cell types present in the tumor microenvironment, although recent developments indicate that tumor associated lymphocytes and fibroblasts can be included in the culture to provide an even better reflection of the original tumor $(55,56)$.

In the past two decades, several pro-inflammatory cytokines have been shown to be increased in the circulation of tumor-bearing hosts, and they are now widely accepted to be major drivers of cancer cachexia (45). Nevertheless, it is still largely unknown which tissues and cell types are responsible for the increase in circulating cytokines in cancer cachexia, despite evidence that tumors are an important source (57). To our surprise, IL-6, which has emerged as a critical cytokine related to the maintenance of body mass (58-60), was only secreted in detectable amounts by the organoids derived from cachectic patients PANCO-6 and PANCO-9. In contrast, variable levels of IL-6 were readily measured in the plasma of both cachectic and non-cachectic patients. This suggests that tumor cells may not be the main source of circulating IL- 6 in patients with pancreatic cancer. Consistent with our data, Öhlund et. al recently showed that pancreatic tumor organoids did not secrete detectable levels of IL-6 whereas increased IL- 6 levels were measured when tumor organoids and cancer-associated fibroblasts were co-cultured in a trans-well system (61). Besides cancer-associated fibroblasts, peripheral blood mononuclear cells (PBMCs) might also be important sources of IL- 6 in cachectic patients. PBMCs from cachectic pancreatic cancer patients stimulated with the secretome of IL- 6 producing pancreatic cancer cell lines have been shown to produce more IL- 6 mRNA compared to PBMCs from non-cachectic patients or healthy controls (62). More recently, Moses et al. showed that PBMCs from cachectic patients are primed to produce significantly higher levels of IL- 6 when compared to PBMCs from healthy controls (63). Thus, tumor-derived factors may promote IL-6 secretion through interactions with cells in the micro-environment or with distant tissues or cells. 
In contrast to IL-6, high levels of IL-8 were secreted by all pancreatic tumor organoids. This is in line with a recent study from Callaway and coworkers who showed that IL-8 was released at high rates from human pancreatic cancer cell lines, including primary human pancreatic cancer cells (64). In addition, there is emerging evidence that IL-8 is strongly associated with worse survival of cancer patients and with muscle wasting in patients with different tumor types $(65,66)$. Increased concentrations of IL-8 have been detected in the serum of cachectic compared to non-cachectic patients, and treatment of C2C12 myotubes with recombinant IL-8 was sufficient to induce myotube atrophy, reinforcing its potential as a new mediator of cancer cachexia (67). In the current study, pancreatic tumor organoids of cachectic patients showed the highest secretion of IL-8, highlighting the potential of the organoid model to study the direct effects of tumor-derived factors on target cells. Next to IL-8, GDF15 has gained considerable attention in the cachexia field ever since Johnen et. al discovered dramatic weight loss in mice bearing tumors engineered to overexpress GDF15 (68). GDF15 is one of the key regulators of lean body mass and body weight, and several studies have shown increased levels of GDF15 in the serum of cachectic vs. non-cachectic patients (69-71), suggesting that GDF15 is a potential novel target for treating cancer cachexia. Interestingly, we observed that pancreatic tumor organoids of cachectic patients produced significantly more GDF15 than those of non-cachectic patients. This suggests that pancreatic tumor cell-derived GDF15 may directly or indirectly contribute to tissue wasting in cachexia. However, the number of organoid cultures studied here is still relatively small and the significance of the observed increases and decreases of cachexia-related factors in organoids from cachectic versus non-cachectic patients should be confirmed by expanding the biobank as well as by functional studies with relevant target cells or animal models.

In this study, we used the international consensus definition to define cancer cachexia (3). However, several slightly different definitions of cancer cachexia have been published (3, $72,73)$, underscoring that diagnosing cancer cachexia remains a challenge both in research and in clinical practice. In line with this notion, we encountered difficulties in categorizing patients into the cachectic vs. the non-cachectic group. Whereas the international consensus definition is predominantly based on body weight loss, other criteria often used to classify cachexia, such as decreased muscle strength and abnormal biochemistry (high CRP, low albumin, low hemoglobin) (73), were heterogeneously presented by the patients in our study. Interestingly, Vanhoutte et. al compared two definitions of cachexia using different diagnostic guidelines applied on the same patient population and showed that putting the focus on weight loss overrates the assignment of the diagnosis of cachexia resulting in survival rates with less prognostic value (74). Thorough screening of cachexiarelated parameters and factors, as was performed in the current study, will be essential to get insight into the cachexia status of the patient and will ultimately aid in the translation of in vitro findings to the actual cachectic status of the patient.

In conclusion, our systematic approach of assessing the cachexia status of a patient before establishing and characterizing tumor organoid cultures has generated an organoid biobank which has the potential to be a valuable tool for increasing our understanding of the 
mechanisms driving human cancer-induced cachexia. The organoid biobank will be made available for academic research upon reasonable request to support the use of human preclinical models in translational research. Our current research focuses on the functional impact of organoid-derived factors on various cell types implicated in the pathogenesis of cachexia using in vitro as well as mouse models. These experiments could be the prelude to proteomics- and genomics-based identification of tumor factors that drive key processes underlying cachexia-related metabolic changes. In addition, our ongoing expansion of the number of organoid cultures established from well-phenotyped patients will enable more robust analyses of the differences in expression of cachexia-related factors by organoids from cachectic versus non-cachectic patients. 


\section{References}

1. Bray F, Ferlay J, Soerjomataram I, Siegel RL, Torre LA, Jemal A. Global cancer statistics 2018: GLOBOCAN estimates of incidence and mortality worldwide for 36 cancers in 185 countries. CA Cancer J Clin. 2018;68(6):394-424.

2. Kleeff J, Korc M, Apte M, La Vecchia C, Johnson CD, Biankin AV, et al. Pancreatic cancer. Nat Rev Dis Primers. 2016;2:16022.

3. Fearon K, Strasser F, Anker SD, Bosaeus I, Bruera E, Fainsinger RL, et al. Definition and classification of cancer cachexia: an international consensus. Lancet Oncol. 2011;12(5):48995.

4. Mueller TC, Bachmann J, Prokopchuk O, Friess H, Martignoni ME. Molecular pathways leading to loss of skeletal muscle mass in cancer cachexia--can findings from animal models be translated to humans? BMC Cancer. 2016;16:75.

5. Fearon K, Arends J, Baracos V. Understanding the mechanisms and treatment options in cancer cachexia. Nat Rev Clin Oncol. 2013;10(2):90-9.

6. Mueller TC, Burmeister MA, Bachmann J, Martignoni ME. Cachexia and pancreatic cancer: are there treatment options? World J Gastroenterol. 2014;20(28):9361-73.

7. Dev R, Wong A, Hui D, Bruera E. The Evolving Approach to Management of Cancer Cachexia. Oncology (Williston Park). 2017;31(1):23-32.

8. Acharyya S, Ladner KJ, Nelsen LL, Damrauer J, Reiser PJ, Swoap S, et al. Cancer cachexia is regulated by selective targeting of skeletal muscle gene products. J Clin Invest. 2004;114(3):370-8.

9. Bonetto A, Rupert JE, Barreto R, Zimmers TA. The Colon-26 Carcinoma Tumor-bearing Mouse as a Model for the Study of Cancer Cachexia. J Vis Exp. 2016(117).

10. Brown JL, Lee DE, Rosa-Caldwell ME, Brown LA, Perry RA, Haynie WS, et al. Protein imbalance in the development of skeletal muscle wasting in tumour-bearing mice. $J$ Cachexia Sarcopenia Muscle. 2018;9(5):987-1002.

11. Judge SM, Wu CL, Beharry AW, Roberts BM, Ferreira LF, Kandarian SC, et al. Genome-wide identification of FoxO-dependent gene networks in skeletal muscle during $\mathrm{C} 26$ cancer cachexia. BMC Cancer. 2014;14:997.

12. Kir S, White JP, Kleiner S, Kazak L, Cohen P, Baracos VE, et al. Tumour-derived PTH-related protein triggers adipose tissue browning and cancer cachexia. Nature. 2014;513(7516):1004.

13. Delitto D, Judge SM, Delitto AE, Nosacka RL, Rocha FG, DiVita BB, et al. Human pancreatic cancer xenografts recapitulate key aspects of cancer cachexia. Oncotarget. 2017;8(1):117789.

14. Parajuli P, Kumar S, Loumaye A, Singh P, Eragamreddy S, Nguyen TL, et al. Twist1 Activation in Muscle Progenitor Cells Causes Muscle Loss Akin to Cancer Cachexia. Dev Cell. 2018;45(6):712-25 e6.

15. Togashi Y, Kogita A, Sakamoto H, Hayashi H, Terashima M, de Velasco MA, et al. Activin signal promotes cancer progression and is involved in cachexia in a subset of pancreatic cancer. Cancer Lett. 2015;356(2 Pt B):819-27.

16. Baker BM, Chen CS. Deconstructing the third dimension: how 3D culture microenvironments alter cellular cues. J Cell Sci. 2012;125(Pt 13):3015-24.

17. Ben-David U, Siranosian B, Ha G, Tang H, Oren Y, Hinohara K, et al. Genetic and transcriptional evolution alters cancer cell line drug response. Nature. 2018;560(7718):32530.

18. Boj SF, Hwang Cl, Baker LA, Chio, II, Engle DD, Corbo V, et al. Organoid models of human and mouse ductal pancreatic cancer. Cell. 2015;160(1-2):324-38.

19. Clevers H. Modeling Development and Disease with Organoids. Cell. 2016;165(7):1586-97. 
20. Lancaster MA, Huch M. Disease modelling in human organoids. Dis Model Mech. 2019;12(7).

21. Sachs N, de Ligt J, Kopper O, Gogola E, Bounova G, Weeber F, et al. A Living Biobank of Breast Cancer Organoids Captures Disease Heterogeneity. Cell. 2018;172(1-2):373-86 e10.

22. van de Wetering $M$, Francies HE, Francis JM, Bounova $G$, lorio F, Pronk $A$, et al. Prospective derivation of a living organoid biobank of colorectal cancer patients. Cell. 2015;161(4):93345.

23. Yan HHN, Siu HC, Law S, Ho SL, Yue SSK, Tsui WY, et al. A Comprehensive Human Gastric Cancer Organoid Biobank Captures Tumor Subtype Heterogeneity and Enables Therapeutic Screening. Cell Stem Cell. 2018;23(6):882-97 e11.

24. Ponz-Sarvise $M$, Corbo V, Tiriac $H$, Engle DD, Frese KK, Oni TE, et al. Identification of Resistance Pathways Specific to Malignancy Using Organoid Models of Pancreatic Cancer. Clin Cancer Res. 2019;25(22):6742-55.

25. Scognamiglio G, De Chiara A, Parafioriti A, Armiraglio E, Fazioli F, Gallo M, et al. Patientderived organoids as a potential model to predict response to PD-1/PD-L1 checkpoint inhibitors. Br J Cancer. 2019.

26. Weeber F, Ooft SN, Dijkstra KK, Voest EE. Tumor Organoids as a Pre-clinical Cancer Model for Drug Discovery. Cell Chem Biol. 2017;24(9):1092-100.

27. Frisancho AR. New norms of upper limb fat and muscle areas for assessment of nutritional status. Am J Clin Nutr. 1981;34(11):2540-5.

28. Dodds RM, Syddall HE, Cooper R, Benzeval M, Deary IJ, Dennison EM, et al. Grip strength across the life course: normative data from twelve British studies. PLoS One. 2014;9(12):e113637.

29. Ottery FD. Definition of standardized nutritional assessment and interventional pathways in oncology. Nutrition. 1996;12:S15-9.

30. Elia M. The 'MUST' report. Nutritional screening for adults: a multidisciplinary responsibility. Development and use of the 'Malnutrition Universal Screening Tool' (MUST) for adults. British Association for Parenteral and Enteral Nutrition (BAPEN). 2003.

31. Mourtzakis M, Prado CM, Lieffers JR, Reiman T, McCargar LJ, Baracos VE. A practical and precise approach to quantification of body composition in cancer patients using computed tomography images acquired during routine care. Appl Physiol Nutr Metab. 2008;33(5):9971006.

32. van Dijk DP, Bakens MJ, Coolsen MM, Rensen SS, van Dam RM, Bours MJ, et al. Low skeletal muscle radiation attenuation and visceral adiposity are associated with overall survival and surgical site infections in patients with pancreatic cancer. J Cachexia Sarcopenia Muscle. 2017;8(2):317-26.

33. Broutier L, Andersson-Rolf A, Hindley CJ, Boj SF, Clevers H, Koo BK, et al. Culture and establishment of self-renewing human and mouse adult liver and pancreas 3D organoids and their genetic manipulation. Nat Protoc. 2016;11(9):1724-43.

34. Hiatt JB, Pritchard CC, Salipante SJ, O'Roak BJ, Shendure J. Single molecule molecular inversion probes for targeted, high-accuracy detection of low-frequency variation. Genome Res. 2013;23(5):843-54.

35. Ruijter JM, Ramakers C, Hoogaars WM, Karlen $\mathrm{Y}$, Bakker $\mathrm{O}$, van den Hoff MJ, et al. Amplification efficiency: linking baseline and bias in the analysis of quantitative PCR data. Nucleic Acids Res. 2009;37(6):e45.

36. Dentener MA, Bazil V, Von Asmuth EJ, Ceska M, Buurman WA. Involvement of CD14 in lipopolysaccharide-induced tumor necrosis factor-alpha, IL-6 and IL-8 release by human monocytes and alveolar macrophages. J Immunol. 1993;150(7):2885-91.

37. Dev R. Measuring cachexia-diagnostic criteria. Ann Palliat Med. 2019;8(1):24-32.

38. Seino T, Kawasaki S, Shimokawa M, Tamagawa H, Toshimitsu K, Fujii M, et al. Human Pancreatic Tumor Organoids Reveal Loss of Stem Cell Niche Factor Dependence during Disease Progression. Cell Stem Cell. 2018;22(3):454-67 e6. 
39. Nagtegaal ID, Odze RD, Klimstra D, Paradis V, Rugge M, Schirmacher P, et al. The 2019 WHO classification of tumours of the digestive system. Histopathology. 2019.

40. Fitzgerald TL, Hickner ZJ, Schmitz M, Kort EJ. Changing incidence of pancreatic neoplasms: a 16-year review of statewide tumor registry. Pancreas. 2008;37(2):134-8.

41. Simone CG, Zuluaga Toro T, Chan E, Feely MM, Trevino JG, George TJ, Jr. Characteristics and outcomes of adenosquamous carcinoma of the pancreas. Gastrointest Cancer Res. 2013;6(3):75-9.

42. Dunne RF, Hezel AF. Genetics and Biology of Pancreatic Ductal Adenocarcinoma. Hematol Oncol Clin North Am. 2015;29(4):595-608.

43. Krasinskas AM, Moser AJ, Saka B, Adsay NV, Chiosea SI. KRAS mutant allele-specific imbalance is associated with worse prognosis in pancreatic cancer and progression to undifferentiated carcinoma of the pancreas. Mod Pathol. 2013;26(10):1346-54.

44. Collisson EA, Bailey P, Chang DK, Biankin AV. Molecular subtypes of pancreatic cancer. Nat Rev Gastroenterol Hepatol. 2019;16(4):207-20.

45. Baracos VE, Martin L, Korc M, Guttridge DC, Fearon KCH. Cancer-associated cachexia. Nat Rev Dis Primers. 2018;4:17105.

46. Romero-Calvo I, Weber CR, Ray M, Brown M, Kirby K, Nandi RK, et al. Human Organoids Share Structural and Genetic Features with Primary Pancreatic Adenocarcinoma Tumors. Mol Cancer Res. 2019;17(1):70-83.

47. Tiriac H, Belleau P, Engle DD, Plenker D, Deschenes A, Somerville TDD, et al. Organoid Profiling Identifies Common Responders to Chemotherapy in Pancreatic Cancer. Cancer Discov. 2018;8(9):1112-29.

48. Duval K, Grover H, Han LH, Mou Y, Pegoraro AF, Fredberg J, et al. Modeling Physiological Events in 2D vs. 3D Cell Culture. Physiology (Bethesda). 2017;32(4):266-77.

49. Nunes AS, Barros AS, Costa EC, Moreira AF, Correia IJ. 3D tumor spheroids as in vitro models to mimic in vivo human solid tumors resistance to therapeutic drugs. Biotechnol Bioeng. 2019;116(1):206-26.

50. Sato T, Vries RG, Snippert HJ, van de Wetering M, Barker N, Stange DE, et al. Single Lgr5 stem cells build crypt-villus structures in vitro without a mesenchymal niche. Nature. 2009;459(7244):262-5.

51. Ballaro R, Costelli P, Penna F. Animal models for cancer cachexia. Curr Opin Support Palliat Care. 2016;10(4):281-7.

52. Talbert EE, Cuitino MC, Ladner KJ, Rajasekerea PV, Siebert M, Shakya R, et al. Modeling Human Cancer-induced Cachexia. Cell Rep. 2019;28(6):1612-22 e4.

53. Gerber MH, Underwood PW, Judge SM, Delitto D, Delitto AE, Nosacka RL, et al. Local and Systemic Cytokine Profiling for Pancreatic Ductal Adenocarcinoma to Study Cancer Cachexia in an Era of Precision Medicine. Int J Mol Sci. 2018;19(12).

54. Go KL, Delitto D, Judge SM, Gerber MH, George TJ, Jr., Behrns KE, et al. Orthotopic PatientDerived Pancreatic Cancer Xenografts Engraft Into the Pancreatic Parenchyma, Metastasize, and Induce Muscle Wasting to Recapitulate the Human Disease. Pancreas. 2017;46(6):8139.

55. Cattaneo CM, Dijkstra KK, Fanchi LF, Kelderman S, Kaing S, van Rooij N, et al. Tumor organoid-T-cell coculture systems. Nat Protoc. 2020;15(1):15-39.

56. Neal JT, Li X, Zhu J, Giangarra V, Grzeskowiak CL, Ju J, et al. Organoid Modeling of the Tumor Immune Microenvironment. Cell. 2018;175(7):1972-88 e16.

57. Porporato PE. Understanding cachexia as a cancer metabolism syndrome. Oncogenesis. 2016;5:e200.

58. Carson JA, Baltgalvis KA. Interleukin 6 as a key regulator of muscle mass during cachexia. Exerc Sport Sci Rev. 2010;38(4):168-76. 
59. Fujimoto-Ouchi K, Tamura S, Mori K, Tanaka Y, Ishitsuka H. Establishment and characterization of cachexia-inducing and -non-inducing clones of murine colon 26 carcinoma. Int J Cancer. 1995;61(4):522-8.

60. Han J, Lu C, Meng $Q$, Halim A, Yean TJ, Wu G. Plasma concentration of interleukin- 6 was upregulated in cancer cachexia patients and was positively correlated with plasma free fatty acid in female patients. Nutr Metab (Lond). 2019;16:80.

61. Ohlund D, Handly-Santana A, Biffi G, Elyada E, Almeida AS, Ponz-Sarvise M, et al. Distinct populations of inflammatory fibroblasts and myofibroblasts in pancreatic cancer. J Exp Med. 2017;214(3):579-96.

62. Martignoni ME, Kunze P, Hildebrandt $W$, Kunzli B, Berberat $P$, Giese $T$, et al. Role of mononuclear cells and inflammatory cytokines in pancreatic cancer-related cachexia. Clin Cancer Res. 2005;11(16):5802-8.

63. Moses AG, Maingay J, Sangster K, Fearon KC, Ross JA. Pro-inflammatory cytokine release by peripheral blood mononuclear cells from patients with advanced pancreatic cancer: relationship to acute phase response and survival. Oncol Rep. 2009;21(4):1091-5.

64. Callaway CS, Delitto AE, Patel R, Nosacka RL, D'Lugos AC, Delitto D, et al. IL-8 Released from Human Pancreatic Cancer and Tumor-Associated Stromal Cells Signals through a CXCR2ERK1/2 Axis to Induce Muscle Atrophy. Cancers (Basel). 2019;11(12).

65. Hou YC, Wang CJ, Chao YJ, Chen HY, Wang HC, Tung HL, et al. Elevated Serum Interleukin-8 Level Correlates with Cancer-Related Cachexia and Sarcopenia: An Indicator for Pancreatic Cancer Outcomes. J Clin Med. 2018;7(12).

66. Sanmamed MF, Carranza-Rua O, Alfaro C, Onate C, Martin-Algarra S, Perez G, et al. Serum interleukin-8 reflects tumor burden and treatment response across malignancies of multiple tissue origins. Clin Cancer Res. 2014;20(22):5697-707.

67. Cury SS, de Moraes D, Freire PP, de Oliveira G, Marques DVP, Fernandez GJ, et al. Tumor Transcriptome Reveals High Expression of IL-8 in Non-Small Cell Lung Cancer Patients with Low Pectoralis Muscle Area and Reduced Survival. Cancers (Basel). 2019;11(9).

68. Johnen $\mathrm{H}$, Lin S, Kuffner T, Brown DA, Tsai VW, Bauskin AR, et al. Tumor-induced anorexia and weight loss are mediated by the TGF-beta superfamily cytokine MIC-1. Nat Med. 2007;13(11):1333-40.

69. Lerner L, Hayes TG, Tao N, Krieger B, Feng B, Wu Z, et al. Plasma growth differentiation factor 15 is associated with weight loss and mortality in cancer patients. J Cachexia Sarcopenia Muscle. 2015;6(4):317-24.

70. Lerner L, Tao J, Liu Q, Nicoletti R, Feng B, Krieger B, et al. MAP3K11/GDF15 axis is a critical driver of cancer cachexia. J Cachexia Sarcopenia Muscle. 2016;7(4):467-82.

71. Tsai VW, Brown DA, Breit SN. Targeting the divergent TGFbeta superfamily cytokine MIC1/GDF15 for therapy of anorexia/cachexia syndromes. Curr Opin Support Palliat Care. 2018;12(4):404-9.

72. Bozzetti F, Mariani L. Defining and classifying cancer cachexia: a proposal by the SCRINIO Working Group. JPEN J Parenter Enteral Nutr. 2009;33(4):361-7.

73. Evans WJ, Morley JE, Argiles J, Bales C, Baracos V, Guttridge D, et al. Cachexia: a new definition. Clin Nutr. 2008;27(6):793-9.

74. Vanhoutte G, van de Wiel M, Wouters K, Sels M, Bartolomeeussen L, De Keersmaecker S, et al. Cachexia in cancer: what is in the definition? BMJ Open Gastroenterol. 2016;3(1):e000097. 


\section{Supplemental Figures}

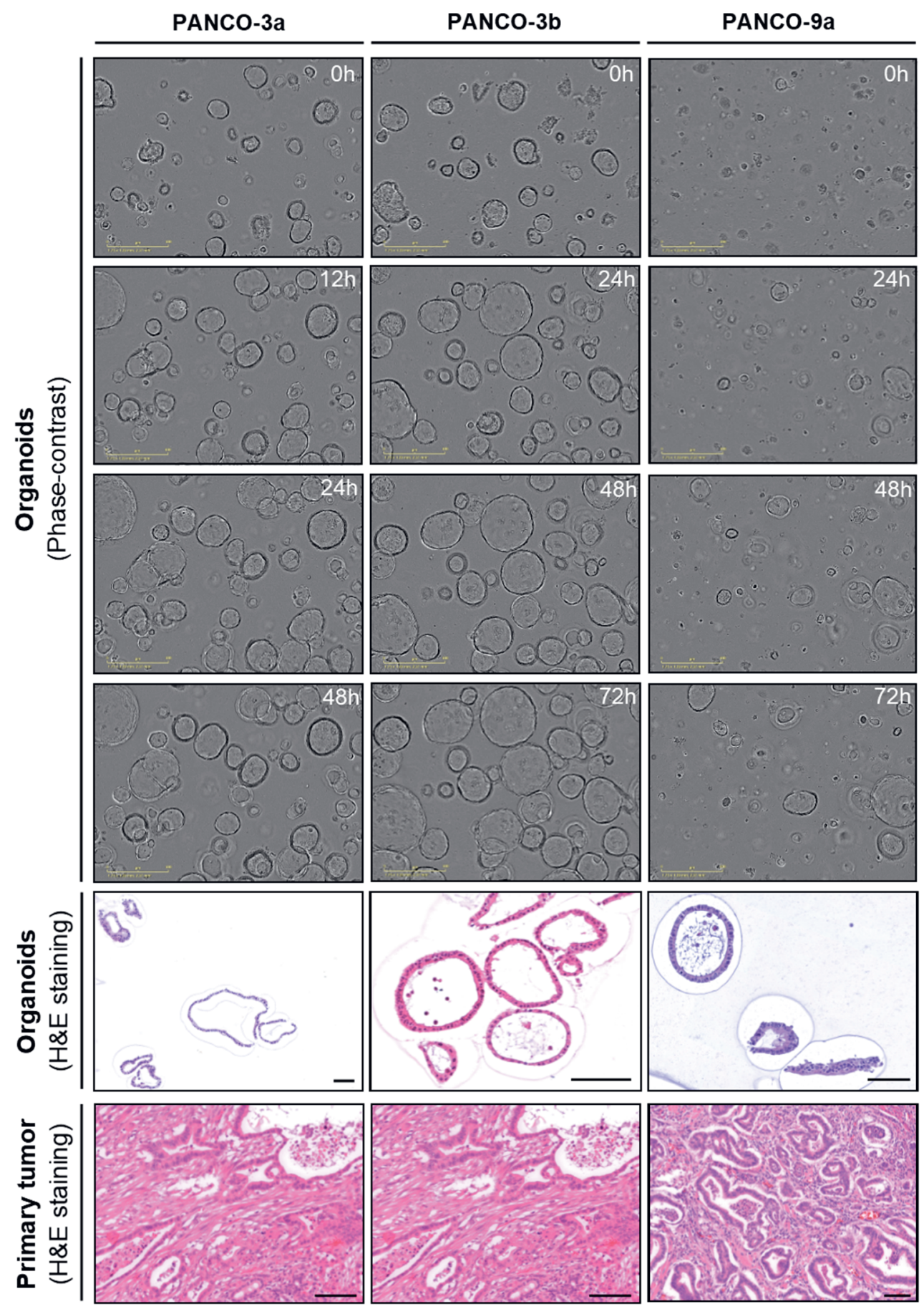




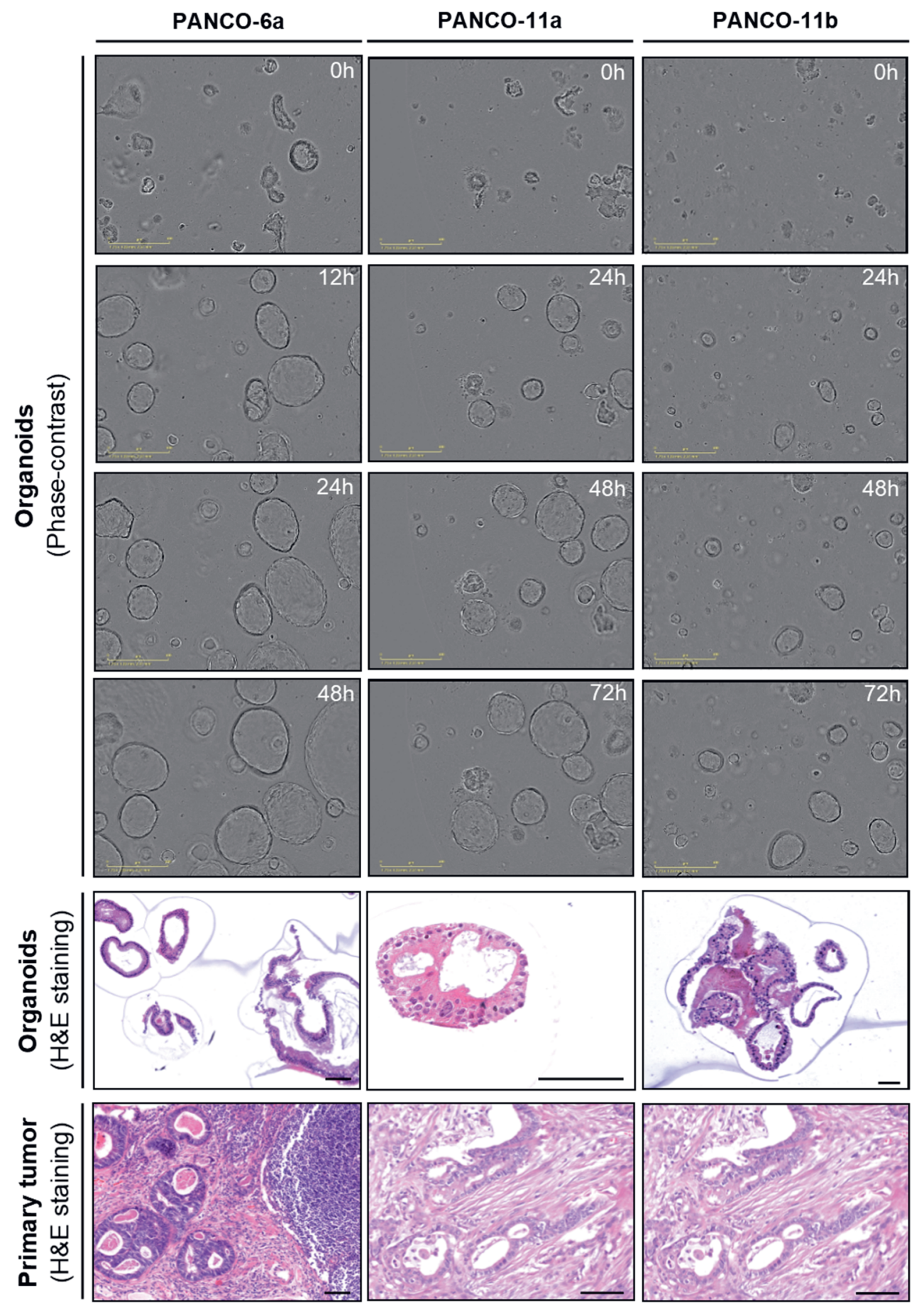


Modeling human pancreatic cancer-induced cachexia with organoids

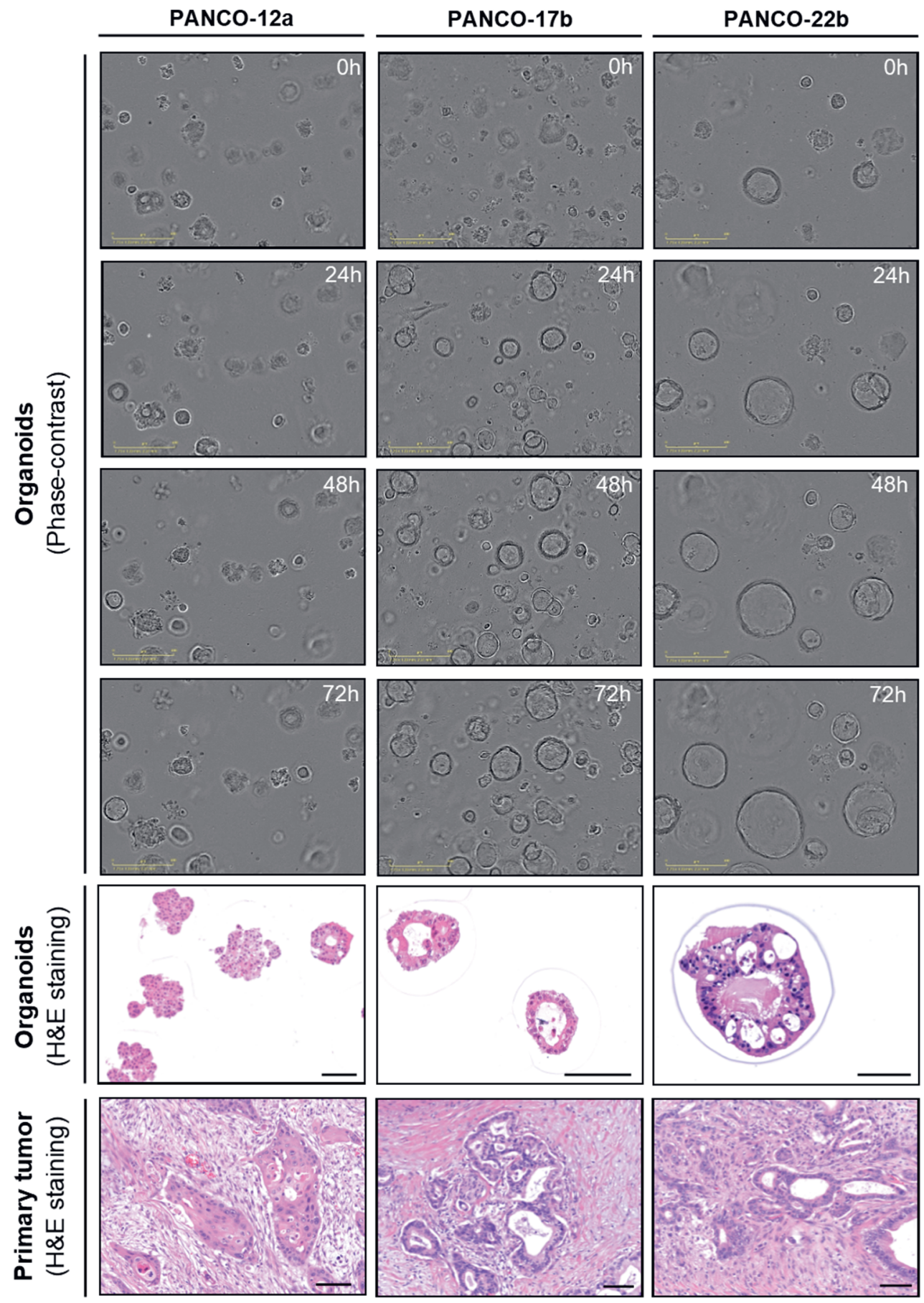




\section{Supplemental Tables}

Table S1: Organoid growth medium

\begin{tabular}{|c|c|c|c|}
\hline Components & Cat. No. & Medium 'a' & Medium 'b' \\
\hline Adv. DMEM/F12 +++ & Gibco $^{\circledR}, \# 12634-010$ & $75 \%(v / v)$ & $25 \%(v / v)$ \\
\hline Noggin CM & Conditioned medium ${ }^{*}$ & $10 \%(v / v)$ & $10 \%(v / v)$ \\
\hline Rspo1 CM & Conditioned medium $^{*}$ & $10 \%(v / v)$ & $10 \%(v / v)$ \\
\hline Wnt3a CM & Conditioned medium $^{*}$ & - & $50 \%(v / v)$ \\
\hline B27 supplement & Gibco $^{\circledR}, \# 17504-044$ & $1: 50$ & $1: 50$ \\
\hline EGF Recombinant Mouse Protein & Gibco $^{\circledR}$, \#PMG8043 & $5 \mathrm{ng} / \mathrm{ml}$ & - \\
\hline $\mathrm{N}$-acetyl cysteine & Sigma-Aldrich, \#A9165 & $1.25 \mathrm{mM}$ & $1.25 \mathrm{mM}$ \\
\hline Primocin & InvivoGen, \#ant-pm-1 & $1: 500$ & $1: 500$ \\
\hline Nicotinamide & Sigma-Aldrich, \#N0636 & $10 \mathrm{mM}$ & $10 \mathrm{mM}$ \\
\hline A83.01 & Sigma-Aldrich, \#SML0788 & - & $500 \mathrm{nM}$ \\
\hline FGF10 & Sino-Biological, \#10573-HNAE & $100 \mathrm{ng} / \mathrm{ml}$ & $100 \mathrm{ng} / \mathrm{ml}$ \\
\hline Gastrin & Sigma-Aldrich, \#G9145 & $10 \mathrm{nM}$ & $10 \mathrm{nM}$ \\
\hline Rhokinase inhibitor Y-27632 & AbMole Bioscience, \#M1817 & $10 \mu \mathrm{M}$ & $10 \mu \mathrm{M}$ \\
\hline
\end{tabular}

* Noggin, Rspondin-1 (Rspo1), and Wnt3a conditioned medium (CM) were produced as described previously (33) 
Table S2: Composition of smMIPs panel*

\begin{tabular}{|c|c|}
\hline Gene & Exon number [positions analyzed for variants] \\
\hline AMELX & $E 6[-61 . .-373]$ \\
\hline ASXL1 & E13[-29..-1929] \\
\hline BRAF & E15[-35..7] \\
\hline CALR & E9[-39..-522] \\
\hline CBL & E8[-79..61]; E9[-20..21] \\
\hline CSF3R & E14[-73..71]; E17[-84..-278] \\
\hline DNMT3A & $\begin{array}{l}\text { E2[143..54]; E3[-70..49]; E4[-59..120]; E5[-32..22]; E6[-47..87]; E7[-64..20]; E8[-41..86]; } \\
\text { E9[-37..36]; E10[-20..82]; E11[-21...],E12[..66]; E13[-19..77]; E14[-80..70]; E15[-27..46]; } \\
\text { E16[-69..45]; E17[-53..86]; E18[-25..56];E19[-20..35];E20[-62..62];E21[-33..37];E22[- } \\
\text { 41..36]; E23[-86..-1262] }\end{array}$ \\
\hline ETNK1 & $\mathrm{E} 3[2 . .-23]$ \\
\hline $\mathrm{EZH} 2$ & $\begin{array}{l}\text { E2[-66..36]; E3[-63..39]; E4[-51..75]; E5[-87..59]; E6[-28..23];E7[-49..37]; E8[-58..35]; E9[- } \\
\text { 62..35]; E10[-78..41]; E11[-52..30]; E12[-23..41];E13[-41..31];E14[-49..21];E15[-57..39]; } \\
\text { E16[-47..22];E17[-37..76];E18[-23..40];E19[-46..47];E20[-82..-203] }\end{array}$ \\
\hline FLT3 & $\mathrm{E} 20[53 . .41]$ \\
\hline IDH1 & E4[243..60] \\
\hline IDH2 & $\mathrm{E} 4[-19 . .53]$ \\
\hline JAK2 & $\mathrm{E} 12[-42 . .33] ; \mathrm{E} 14[6 . .66]$ \\
\hline KIT & E8[-65..-56]; E17[19..4] \\
\hline KRAS & $E 2[-35 . .-3] ; E 3[-17 . .-65]$ \\
\hline MPL & $\mathrm{E} 10[-19 . .72]$ \\
\hline MYD88 & $E 4[-59 . .4] ; E 5[-10 . .-1789]$ \\
\hline NOTCH1 & E34[1287..-1690] \\
\hline NPM1 & $\mathrm{E} 11[-81 . .-501]$ \\
\hline NRAS & $E 2[-32 . .-14] ; E 3[-4 . .-65]$ \\
\hline RUNX1 & $\begin{array}{l}\text { E1[-20..20]; E2[-2..71]; E3[-75..80]; E4[-71..25]; E5[-57..33]; E6[-39..38]; E7[-95..45]; E8[- } \\
\text { 25..20]; E9[-116..-4279] }\end{array}$ \\
\hline SETBP1 & E4[2001..-1205] \\
\hline SF3B1 & E13[-54..],E14[..26] \\
\hline SF3B1 & E15[-90..],E16[..35] \\
\hline SRSF2 & $\mathrm{E} 1[366 . .12]$ \\
\hline TET2 & $\begin{array}{l}\text { E3[5..62]; E4[-62..59]; E5[-21..68]; E6[-40..20]; E7[-40..41]; E8[-64..39]; E9[-38..19]; E10[- } \\
\text { 27..76]; E11[-34..-3195] }\end{array}$ \\
\hline TP53 & $\begin{array}{l}\text { E2[-4..26]; E3[-57..23]; E4[-75..52]; E5[-61..],E6[..64]; E7[-36..28]; E8[-42..],E9[..44]; E10[- } \\
78 . .16] ; E 11[-49 . .-1114]\end{array}$ \\
\hline U2AF1 & $\mathrm{E} 2[-30 . .-2] ; \mathrm{E} 6[42 . .74]$ \\
\hline WT1 & E7[-63..34]; E9[-43..52] \\
\hline ZFY & E8[491..-3089] \\
\hline ZNF185 & E16[1247..1147] \\
\hline
\end{tabular}

* this panel is normally used in the molecular diagnostics of hemato-oncological disorders but was used for this study because it includes KRAS andTP53, the most frequently mutated genes in pancreatic cancer. 
Table S3: qPCR human primers

\begin{tabular}{|c|c|c|c|}
\hline Gene & Full name & Sense primer $\left(5^{\prime}-3^{\prime}\right)$ & Reverse primer $\left(5^{\prime}-3^{\prime}\right)$ \\
\hline IL1A & Interleukin 1 alpha & $\overline{\text { GCTGAAGGAGATGCCTGAGAT }}$ & TTAGTGCCGTGAGTTTCCCAG \\
\hline$I L 1 B$ & Interleukin 1 beta & CTGAGCTCGCCAGTGAAATG & TTTAGGGCCATCAGCTTCAAA \\
\hline IL6 & Interleukin 6 & TACCCCCAGGAGAAGATTCC & TTTCAGCCATCTTTGGAAGG \\
\hline IL8 & Interleukin 8 & CTGGCCGTGGCTCTCTTG & TTAGCACTCCTTGGCAAAACTG \\
\hline TNFA & Tumor necrosis factor alpha & CCTGCTGCACTTTGGAGTGA & GAGGGTTTGCTACAACATGGG \\
\hline LIF & Leukemia inhibitor factor & GTCTTGGCGGCAGTACACAG & ACGACTATGCGGTACAGCTC \\
\hline GDF15 & Growth differentiation factor 15 & TGGGAAGATTCGAACACCGA & CCCGAGAGATACGCAGGTG \\
\hline MCP1 & Monocyte chemotactic protein 1 & TCACCTGCTGTTATAACTTCAC & CAATGGTCTTGAAGATCACAG \\
\hline CYPA & Cyclophilin A & CTCGAATAAGTTTGACTTGTGTTT & CTAGGCATGGGAGGGAACA \\
\hline$B 2 M$ & Beta-2-microglobulin & TCCATCCGACATTGAAGTTG & CGGCAGGCATACTCATCTT \\
\hline
\end{tabular}




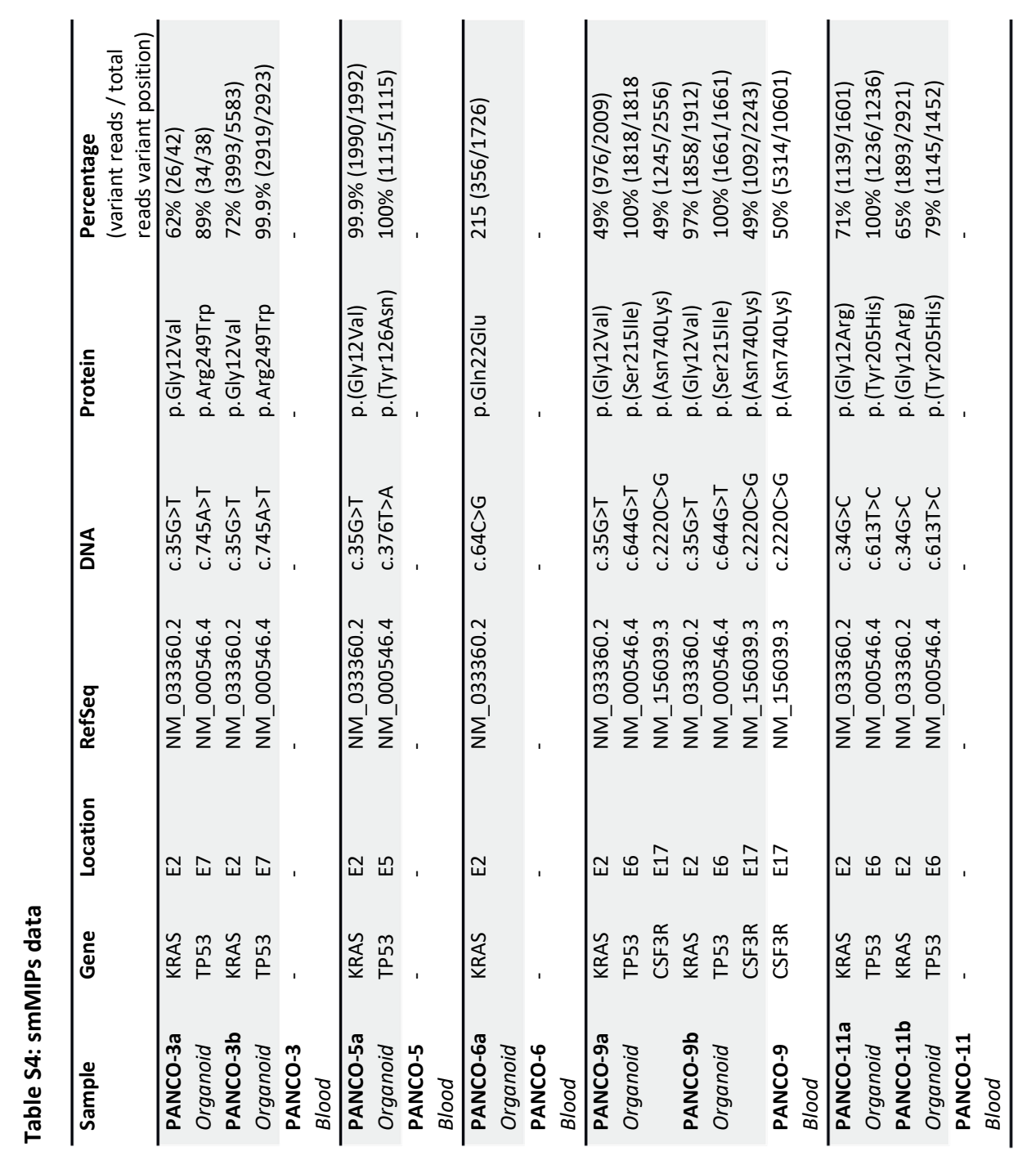




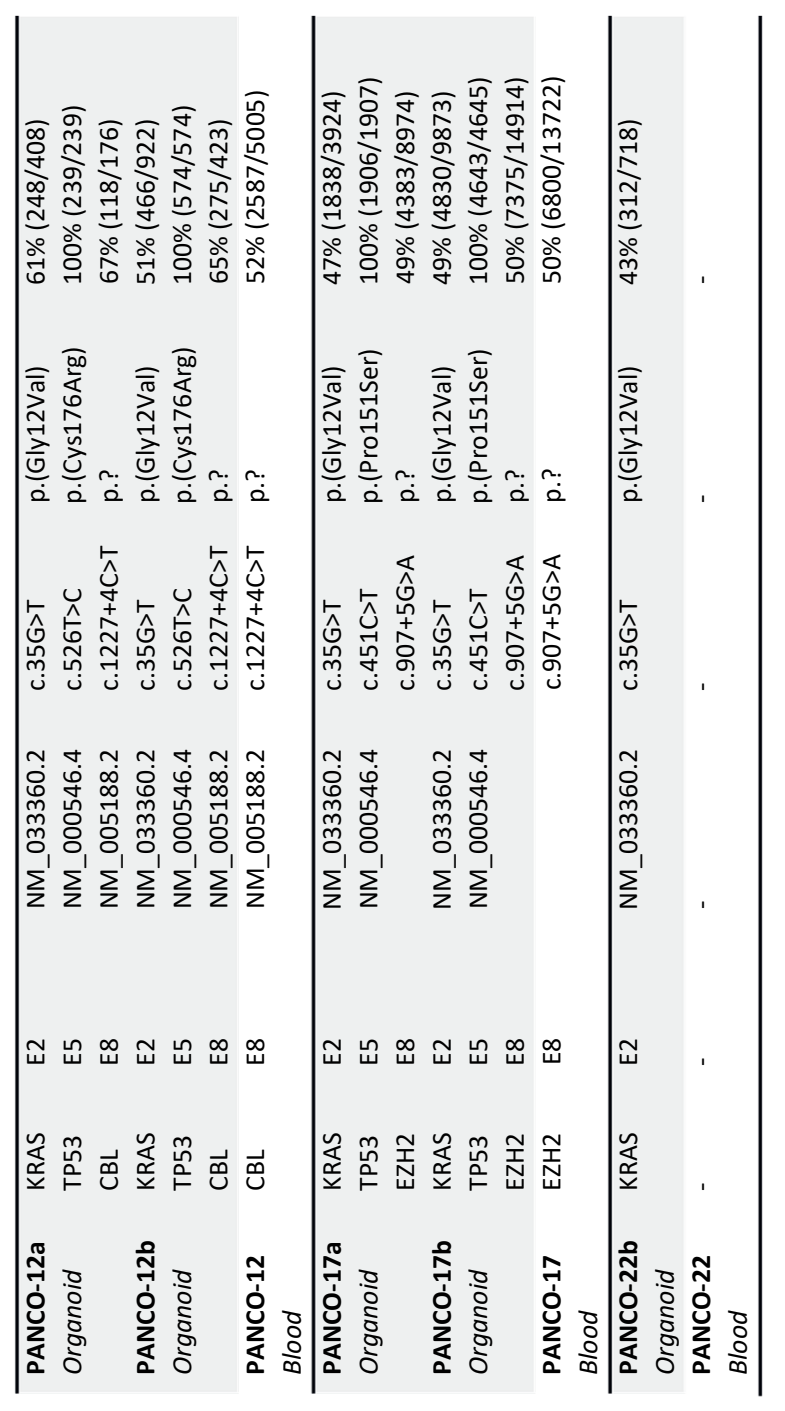


Table S5: KRAS mutation analysis of parent tumors in relation to KRAS mutation status of organoid cultures

\begin{tabular}{lllll}
\hline Sample & $\begin{array}{l}\text { KRAS mutation } \\
\text { Organoid }\end{array}$ & Amino acid change & $\begin{array}{l}\text { KRAS mutation } \\
\text { Primary tumor }\end{array}$ & Amino acid change \\
\hline PANCO-5 & c.35G>A & Gly12Asp (G12D) & $\begin{array}{l}\text { c.35G AND } \\
\text { c.35G }>\text { A }\end{array}$ & Gly12Asp (G12D) \\
\hline PANCO-6 & c.64C $>$ G & Gln22Glu & c.64C & \\
\hline PANCO-9 & c.35G $>\mathrm{T}$ & Gly12Val & c.35G & \\
\hline PANCO-11 & c.34G $>\mathrm{C}$ & Gly12Arg (G12R) & c.34G AND & Gly12Arg (G12R) \\
& & & c.34G $>$ C & \\
\hline PANCO-12 & c.35G $>\mathrm{T}$ & Gly12Val & c.35G & \\
\hline PANCO-17 & c.35G $>\mathrm{T}$ & Gly12Val & Quality too low & \\
\hline PANCO-22 & c.35G $>\mathrm{T}$ & Gly12Val & c.35G & \\
\hline
\end{tabular}

Table S6: TP53 mutation analysis of parent tumors in relation to TP53 mutation status of organoid cultures

\begin{tabular}{lllll}
\hline Sample & $\begin{array}{l}\text { TP53 mutation } \\
\text { Organoid }\end{array}$ & Amino acid change & $\begin{array}{l}\text { TP53 mutation } \\
\text { Primary tumor }\end{array}$ & Amino acid change \\
\hline PANCO-5 & c.376T>A & Try126Asn & Quality too low & \\
\hline PANCO-6 & $\begin{array}{l}\text { No mutation } \\
\text { detected }\end{array}$ & & $\begin{array}{l}\text { No mutation } \\
\text { detected }\end{array}$ & \\
\hline PANCO-9 & c.644G $>$ T & Ser215Ile (S215I) & $\begin{array}{l}\text { c.644G AND } \\
\text { c.644G }>\text { T }\end{array}$ & Ser215Ile (S215I) \\
& c.613T>C & Tyr205His & c.613T & \\
\hline PANCO-11 & c.526T>C & Cys176Arg & c.526T AND & Cys176Arg \\
\hline PANCO-12 & c.526T >C & \\
\hline PANCO-17 & c.451C $>$ T & Pro151Ser & c.451C & \\
\hline PANCO-22 & No mutation & & $\begin{array}{l}\text { No mutation } \\
\text { detected }\end{array}$ & \\
& detected & & & \\
\hline
\end{tabular}




\section{Supplemental videos}

Video S1-S13: Morphological characterization of pancreatic tumor organoids.

The growth of pancreatic tumor organoids was monitored using live cell imaging. Phase-contrast images were acquired every 1-2h. Scale bar $=400 \mu \mathrm{m}$

Video S1 - PANCO-3a

Video S2 - PANCO-3b

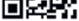

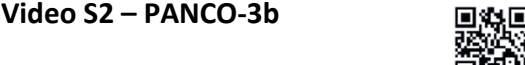

Video S3 - PANCO-5a

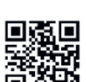

Video S4 - PANCO-6a

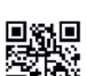

Video S5 - PANCO-9a

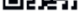

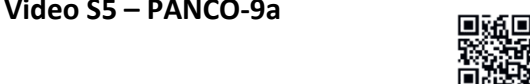

Video S6 - PANCO-9b

Video S7 - PANCO-11a

品此

Video S8 - PANCO-11b

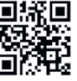

Video S9 - PANCO-12a

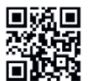

Video S10 - PACNO-12b

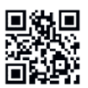

Video S11 - PANCO-17a
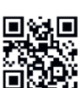

Video S12 - PANCO-17b

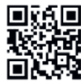

Video S13 - PANCO-22b

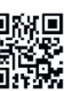




\section{CHAPTER 3}

\section{Human pancreatic tumor organoid-derived factors enhance myogenic differentiation}

Rianne D.W. Vaes, David P.J. van Dijk, Steven W.M. Olde Damink, Sander S. Rensen*, Ramon Langen*

* Shared senior author

Submitted 


\section{Abstract}

Background: Most patients with pancreatic cancer develop cachexia, which is characterized by progressive muscle loss. The mechanisms underlying muscle loss in cancer cachexia remain elusive. Pancreatic tumor organoids are 3D cell culture models that retain key characteristics of the parent tumor. We aimed to investigate the effect of pancreatic tumor organoid-derived factors on processes that determine skeletal muscle mass, including the regulation of muscle protein turnover and myogenesis.

Methods: Conditioned medium (CM) was collected from human pancreatic cancer cell lines (PK-45H, PANC-1, PK-1, and KLM-1) and pancreatic tumor organoid cultures from a severely cachectic (PANCO-9a) and a non-cachectic patient (PANCO-12a). Differentiating C2C12 myoblasts and mature $\mathrm{C} 2 \mathrm{C} 12$ myotubes were exposed to $\mathrm{CM}$ for $24 \mathrm{~h}$ or maintained in control medium. In myotubes, NF-kB activation was monitored using a NF-kB luciferase reporter construct, and mRNA expression of E3-ubiquitin ligases and REDD1 was analyzed by RT-qPCR. C2C12 myoblast proliferation and differentiation was monitored by live cell imaging; 48h after initiation of differentiation, myogenic markers and myosin heavy chain (MyHC) isoforms were assessed by RT-qPCR.

Results: Whereas CM from PK-1 and KLM-1 cells significantly induced NF-KB activation in C2C12 myotubes, Atrogin-1/MAFbx and MuRF1 mRNA were only minimally and inconsistently upregulated by the CM of pancreatic cancer cell lines. Similarly, E3-ubiquitin ligases and REDD1 mRNA expression in myotubes were not altered by exposure to pancreatic tumor organoid-derived factors. CM of both PANCO-9a and PANCO-12a tumor organoids increased proliferation of myoblasts, which was accompanied by significant downregulation of the satellite cell marker paired-box 7 (PAX7) and myogenic factor 5 (MYF5) after $48 \mathrm{~h}$ of differentiation. Live cell imaging revealed accelerated alignment and fusion of myoblasts exposed to CM from PANCO-9a and PANCO-12a, which was in line with increased Myomaker mRNA expression levels. These morphological and transcriptional alterations were accompanied by increased expression of several factors reflecting biochemical muscle differentiation, as well as increased abundance of the glycolytic fasttwitch myofiber MyHC-IIB.

Conclusion: These data show that pancreatic tumor organoid-derived factors alter the kinetics of myogenesis, which may eventually contribute to impaired muscle mass maintenance in cancer cachexia. 


\section{Introduction}

Pancreatic cancer is a highly lethal malignancy that accounts for $4.5 \%$ of all cancer deaths worldwide (1). Despite advances in our understanding of the complex tumor biology of pancreatic cancer and new treatment strategies, the prognosis of pancreatic cancer patients has only marginally improved (2).

A major contributor to the poor survival of pancreatic cancer patients is the cachexia syndrome, which is present in up to $80 \%$ of the patients at the time of diagnosis (3). Cancer cachexia is a severe wasting disorder defined by involuntary loss of muscle mass which cannot be fully reversed by conventional nutritional support and which leads to progressive functional impairment (4). The pathophysiology of cancer cachexia remains to be elucidated but it is well appreciated that it is driven by a complex interplay between tumor- and host-derived factors that contributes to a negative protein and energy balance (3). Since effective targeted therapies to treat cachexia are currently lacking, a better understanding of the cachexia-inducing factors and mechanisms is crucial to prevent its onset and progression and to improve overall survival of pancreatic cancer patients.

Most of our current understanding of the cachexia-inducing factors expressed and released by tumor cells is derived from in vitro and in vivo studies using established cancer cell lines (5-10). In particular, studies with mouse C26-colon carcinoma (C26) and Lewis Lung carcinoma (LLC) cells have shown that tumor-derived factors induce skeletal muscle atrophy by activation of NF-KB and ubiquitin proteasome pathway (UPS)-mediated proteolytic degradation of specific muscle proteins (11-14). Moreover, impairments in myogenesis and its regulation have been implicated in the net loss of skeletal muscle in the context of cachexia (15-17).

Whereas these murine cell lines have contributed tremendously to our current understanding of cancer cachexia mechanisms, their suitability for modeling human cancer cachexia may not be optimal (18). For example, C26 and LLC-tumor bearing mice develop cachexia on a timescale of only a few weeks and their tumor volume typically represents more than $10 \%$ of the entire body mass, which is not the case in humans $(7,14,18)$. As such, human cell lines have been increasingly used to study cancer-induced cachexia $(6,7)$. In particular, human pancreatic cancer cell lines like MiaPaCa-2, Capan-1, and Panc-1 have been frequently applied because of the severity of cachexia in pancreatic cancer $(6,7,19$, 20). Unfortunately, the actual cachexia-related clinical background of these cell lines is unknown. In addition, cell lines are routinely cultured on a stiff two-dimensional (2D) plastic surface in the absence of physiological gradients of oxygen and nutrients and without functional cellular-extracellular matrix interactions (21). These comparatively artificial culture conditions may evoke important non-physiological cellular adaptations that are associated with mutational and chromosomal instability (22). As the majority of the cell lines used to study cancer cachexia mechanisms have been cultured in 2D for decades, currently available strains are likely to be genetically different compared to the originally isolated tumor cells, potentially affecting gene expression patterns. As a result, the established pancreatic cancer cell lines will likely have gained or lost cachexia-inducing 
properties, contributing to inconsistent findings among studies. Taken together, these limitations of existing 2D cell lines highlight the need for better experimental models that contribute to our understanding of human cancer-induced cachexia.

Recently, several aspects of cancer biology have been shown to be accurately modeled by three-dimensional (3D) organoid cultures (23-25). Pancreatic tumor organoids can be efficiently established by culturing primary epithelial tumor cells in basement membrane extract (BME) and a defined, tissue-specific growth medium. These tumor cells self-organize into 3D structures mimicking the architecture of the organ of origin, and have been shown to closely recapitulate pathophysiologically relevant aspects of pancreatic cancer both in vitro and in vivo (23). Hence, we applied pancreatic tumor organoid cultures that were recently established in our lab (26) to investigate cachexia-inducing properties of pancreatic cancer cells. We used tumor organoids from a severely cachectic and from a noncachectic patient to assess the direct effect of tumor-derived factors on C2C12 skeletal muscle cells, focusing on muscle atrophy signaling and myogenesis. We hypothesized that tumor organoid-derived $\mathrm{CM}$ induces myotube atrophy and impairs myogenesis. 


\section{Materials and Methods}

\section{Human pancreatic tumor organoid culture}

Pancreatic tumor organoid cultures PANCO-9a and PANCO-12a were previously generated and characterized in our laboratory (26). In short, ethical approval (METC 13-4-107) was obtained to pre-operatively assess the cachexia status of pancreatic cancer patients undergoing surgical resection. Surgically removed pancreatic tumor tissue was used to generate pancreatic tumor organoids. This study has therefore been performed in accordance with the ethical standards laid down in the 1964 Declaration of Helsinki and its later amendments.

For maintaining cultures, organoids were resuspended in ice-cold basement membrane extract (BME; Geltrex LDEV-Free Reduced Growth Factor Basement Membrane Matrix, Gibco, Cat. No. 1413202) and three 15 $\mu \mathrm{L}$ droplets of Geltrex-cell suspension were allowed to solidify per well of a 24-wells culture plate (Eppendorf,) at $37^{\circ} \mathrm{C}$ for $30 \mathrm{~min}$. When the droplets were solidified, $500 \mu \mathrm{l}$ of medium was added to each well (26). The plate was transferred to a humidified $37^{\circ} \mathrm{C} / 5 \% \mathrm{CO}_{2}$ incubator and medium was changed every $2-3$ days. The organoids were passaged every 7-10 days. Organoids were mechanically sheared, resuspended in ice-cold $\mathrm{BME}$, and re-plated as described above.

\section{Collection of pancreatic tumor organoid conditioned medium}

One day before passaging, organoid growth medium was replaced by basal culture medium consisting of DMEM/F12 supplemented with 1\% (v/v) HEPES and 1\% (v/v) antibiotics (100 units $/ \mathrm{mL}$ penicillin and $100 \mu \mathrm{g} / \mathrm{mL}$ streptomycin, GIBCO). Additional wells containing empty Geltrex-droplets overlaid with basal culture medium were included for the collection of control medium. After $24 \mathrm{~h}$, conditioned medium (CM) was collected and centrifuged at 350 $\mathrm{xg}$ for $10 \mathrm{~min}$ at $4^{\circ} \mathrm{C}$. The supernatant was centrifuged for another $20 \mathrm{~min}$ at $2,000 \mathrm{xg}$ at $4^{\circ} \mathrm{C}$ and the resulting $\mathrm{CM}$ cleared from cellular debris was aliquoted and stored at $-80^{\circ} \mathrm{C}$.

\section{Culturing of 2D pancreatic cancer cell lines and collection of conditioned medium}

Human pancreatic cancer cell lines PK-45H, PANC-1 (27), PK-1 (28), and KLM-1 (29) (obtained from RIKEN BioResource Center) were cultured in high glucose $(4.5 \mathrm{~g} / \mathrm{L})$ Dulbecco's Modified Eagle's Medium (DMEM) supplemented with $10 \%$ (v/v) fetal bovine serum (FBS) (Greiner Bio-one, cat. no. 758093) and 1\% (v/v) antibiotics (100 units/mL penicillin and $100 \mu \mathrm{g} / \mathrm{mL}$ streptomycin, GIBCO). All cell lines were maintained at $37^{\circ} \mathrm{C}, 5 \%$ $\mathrm{CO}_{2}$ in a humidified incubator.

Medium used for the production of CM consisted of DMEM $(10 \% \mathrm{v} / \mathrm{v})$ diluted in Hank's Balanced Salt solution (HBSS) (GIBCO) supplemented with $\mathrm{NaHCO}_{3}$ (Sigma-Aldrich, Saint Louis, MO), 10\% (v/v) FBS, and $1 \%(\mathrm{v} / \mathrm{v})$ antibiotics. The medium was conditioned for $48 \mathrm{~h}$ using cells at $\sim 60-80 \%$ confluency. $\mathrm{CM}$ was collected and stored at $-80^{\circ} \mathrm{C}$. 


\section{C2C12 skeletal muscle cell culture}

Murine $\mathrm{C} 2 \mathrm{C} 12$ skeletal myoblasts (obtained from the American Type Culture Collection, \#CRL1772) were cultured in growth medium (GM), composed of low glucose (1g/L) DMEM supplemented with $10 \%(\mathrm{v} / \mathrm{v})$ FBS and $1 \%(\mathrm{v} / \mathrm{v})$ antibiotics (100 units $/ \mathrm{mL}$ penicillin and 100 $\mu \mathrm{g} / \mathrm{mL}$ streptomycin, $\mathrm{GIBCO}$ ). The cells were maintained at $37^{\circ} \mathrm{C}, 5 \% \mathrm{CO}_{2}$ in a humidified incubator.

Myotubes were generated by plating $\mathrm{C} 2 \mathrm{C} 12$ myoblasts at a density of $1.0 \times 10^{4}$ cells $/ \mathrm{cm}^{2}$ on BD Matrigel-coated (Matrige ${ }^{\circledR}$ Matrix Basement Membrane - Growth factor reduced, Corning) (1:50 in low glucose DMEM) cell culture plates (Eppendorf); myoblasts were cultured in $\mathrm{GM}$ for $48 \mathrm{~h}$ before induction of differentiation. To induce differentiation, GM was replaced by differentiation medium (DM) consisting of DMEM supplemented with $1 \%$ heat-inactivated $\mathrm{FBS}\left(30 \mathrm{~min}\right.$ at $\left.56^{\circ} \mathrm{C}\right)$ and $0.5 \%(\mathrm{v} / \mathrm{v})$ antibiotics $(50 \mathrm{units} / \mathrm{mL}$ penicillin and $50 \mathrm{\mu g} / \mathrm{ml}$ streptomycin, GIBCO). The medium was refreshed every $48 \mathrm{~h}$. At indicated time points, C2C12 skeletal myoblasts or myotubes were exposed to $50 \% \mathrm{v} / \mathrm{v} \mathrm{CM}$ diluted in differentiation medium.

\section{NF-KB-luciferase activity}

At day 5 of differentiation, C2C12 myotubes with a luciferase construct containing 3 tandem $\mathrm{NF}-\mathrm{KB}$ luciferase responsive elements (30) were incubated for $4 \mathrm{~h}$ with $\mathrm{CM}$. For the assessment of NF-KB activation, myotubes were harvested in $1 \mathrm{x}$ luciferase buffer (5x Reporter Lysis Buffer, Promega) on ice and cell lysates were stored at $-80^{\circ} \mathrm{C}$. Luciferase activity was measured according to the manufacturer's protocol (Promega) using a luminometer (Berthold Technologies). Luciferase activity was corrected for total protein by using the BCA Protein assay according to the manufacturer's protocol (Pierce ${ }^{\circledast}$ BCA Protein Assay Kit, Thermo Scientific, Rockford, IL).

\section{Quantitative real-time $P C R$}

Total RNA was extracted from cell cultures using TRI Reagent (Sigma, St. Louis, MO) according to the manufacturer's instructions. RNA was reconstituted in RNase free water and stored at $-80^{\circ} \mathrm{C}$. The RNA concentration was measured using a DeNovix DS-11 spectrophotometer and 375 ng RNA were reversed transcribed using the SensiFast cDNA Synthesis Kit according to the manufacturer's instructions (Bioline $\mathrm{GmbH}$, Germany).

To quantify mRNA expression levels, quantitative real-time PCR (qRT-PCR) analysis was performed on the LightCycler480 (Roche) using a three-step PCR program followed by melt curve analysis. cDNA was amplified with the SensiMix SYBR Hi-Rox Kit (Bioline, cat. No. QT605-05). Specific primer pairs for each gene were ordered from Sigma as listed in table S1. Relative gene expression levels were derived from the LinRegPCR (Version 2016.1) method (31) and normalized to the geometric average of three reference genes, cyclophilin A (CYPA), $\beta$-2-microglobulin (62M), and 60S acidic ribosomal protein PO (RPLPO). 


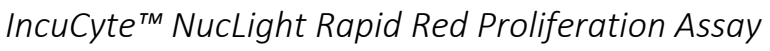

C2C12 myoblasts were plated on Matrigel-coated 96-wells cell culture plates (Eppendorf) at a density of $1 \times 10^{4} \mathrm{cell} / \mathrm{s} / \mathrm{cm}^{2}$. The cells were cultured for $48 \mathrm{~h}$ in GM before the induction of differentiation. The IncuCyte NucLight Rapid Red Reagent (Essen Bioscience, Cat no. 4717) was diluted $1: 250$ in DM containing $2 \%(v / v)$ HI-FBS. Subsequently, DM containing NucLight Rapid Red was diluted with either control (DMEM/F12) (50\% v/v) or CM samples $(50 \% \mathrm{v} / \mathrm{v})$ resulting in a final NucLight Rapid Red dilution of 1:500 and $1 \% \mathrm{HI}-\mathrm{FBS}$ in all conditions. Insulin growth factor 1 (IGF-1) (Sigma), a well-known anabolic stimulus, was included as a positive control and was added at the induction of differentiation. The differentiation of $\mathrm{C} 2 \mathrm{C} 12$ myoblasts into myotubes was monitored through an IncuCyte ${ }^{\circledR} \mathrm{S} 3$ Live-Cell analysis system (Sartorius). Phase-contrast images and red-fluorescence images were captured every two hours using the 10x objective. The integrated red object count metric tool was used to quantify the number of nuclei.

\section{Statistics}

Data are representative of three independent experiments performed in triplicate and are expressed as mean \pm SEM. Raw data were analyzed using IBM SPSS 25 for Microsoft Windows ${ }^{\circledast}$. Statistical analyses were performed using the independent sample T-test to compare differences between two groups. In case of more than two groups, the One-way ANOVA test was used followed by Tukey's post-hoc testing. A p-value of $p<0.05$ was considered statistically significant. 


\section{Results}

Tumor-derived factors from established 2D pancreatic cancer cell lines do not consistently induce NF-KB or atrophy signaling in mature C2C12 myotubes

Previous studies using conventional 2D colon and lung carcinoma lines have demonstrated that tumor-derived factors may directly induce $\mathrm{C} 2 \mathrm{C} 12$ skeletal muscle atrophy by activation of the ubiquitin proteasome pathway (UPS). Furthermore, proteolytic degradation of specific muscle proteins has been shown to be regulated via tumor factor-promoted NF-KB activation (8). Given that pancreatic cancers generally have strong cachexia-inducing abilities, we first investigated whether tumor-derived factors from various established human 2D pancreatic cancer cell lines could initiate skeletal muscle atrophy signaling in vitro. Differentiated $\mathrm{C} 2 \mathrm{C} 12$ myotubes were incubated with $\mathrm{CM}$ from four pancreatic cancer cell lines (PK-45H, PANC-1, PK-1, and KLM-1). First, we assessed the effect of CM on NF-KB activation (Fig. 1A). TNF- $\alpha$, a known inducer of NF-KB signaling and here included as a positive control, significantly induced NF-KB activity (5.0-fold, $\mathrm{p}<0.001)$ in $\mathrm{C} 2 \mathrm{C} 12$ myotubes within $4 \mathrm{~h}$. Whereas CM from PK-1 (3.1-fold, $\mathrm{p}<0.001)$ and KLM-1 (2.1-fold, $\mathrm{p}=0.01)$ also induced $\mathrm{C} 2 \mathrm{C} 12 \mathrm{NF}-\mathrm{KB}$ activation, this was not observed after exposure to $\mathrm{CM}$ from $\mathrm{PK}-45 \mathrm{H}$ and PANC-1. Next, we assessed whether expression of Atrogin-1/MAFbx and MuRF1, muscle-specific E3-ubiquitin ligases involved in muscle proteolysis which have been reported to be upregulated under atrophy-inducing conditions (32), was affected by pancreatic cancer cell line-derived factors (Fig. 1B). Treatment with HBSS to mimic muscle atrophy as a consequence of nutrient deprivation caused significantly increased expression of Atrogin-1/MAFbx (4.2-fold, $\mathrm{p}<0.001)$ and MuRF1 (3.6-fold, $\mathrm{p}<0.001)$, showing the responsiveness of the system. However, increased Atrogin-1/MAFbx mRNA levels were only found after incubation with CM from PK-1 (1.7-fold, $\mathrm{p}=0.015)$, and MURF1 mRNA expression levels were only increased by CM from PK-45H (2.0-fold, $p=0.017)$. Collectively, these data

A

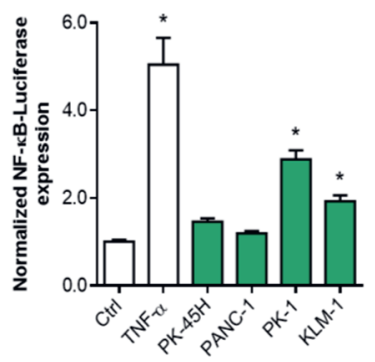

B

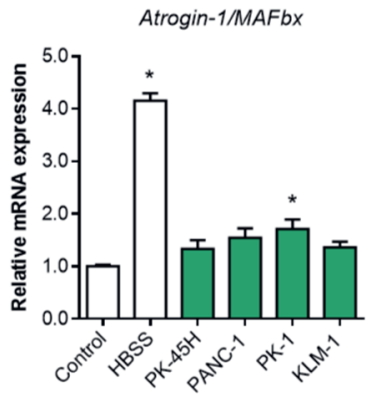

MuRF1

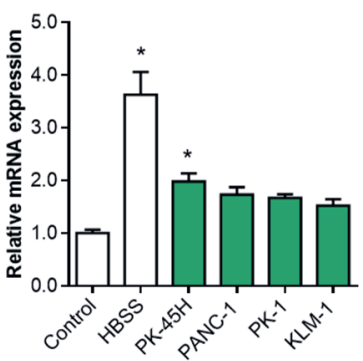

Figure 1: Effect of tumor-derived factors from established 2D pancreatic cancer cell lines on C2C12 myotubes. Mature $\mathrm{C} 2 \mathrm{C} 12$ myotubes were treated with $\mathrm{CM}$ from PK-45H, PANC-1, PK-1, and KLM-1. (A) After 4h, the NF-kB luciferase activity luciferase was assessed. (B) mRNA expression of Atrogin$1 /$ MAFbx and MURF1 were determined after 24h. Data were normalized to CYPA, B2M, and RPLPO reference genes. Relative expression levels are obtained from three independent experiments. Data are presented as mean \pm SEM. 
show that whereas some human pancreatic cancer cell lines display certain skeletal muscle atrophy-inducing properties, their effects are generally small and inconsistent. Moreover, cachexia-related clinical data of the donor patients of these cell lines are not available (2729), questioning their application for modeling pancreatic cancer-induced cachexia.

Human pancreatic tumor organoid-derived factors do not induce skeletal muscle atrophy signaling in mature C2C12 myotubes

Given the limitations of human 2D pancreatic cancer cell lines and promising developments in organoid technology, we recently established an organoid biobank consisting of pancreatic tumor organoid cultures originating from patients whose cachexia phenotype was thoroughly assessed (26). According to the international consensus definition of cancer cachexia, we selected an organoid culture from a cachectic (PANCO-9a) and a non-cachectic (PANCO-12a) patient for the current study. These patients showed substantial differences concerning the percentage of weight loss over the past 6 months (PANCO-9a: $13.4 \%$ vs PANCO-12a: $1.2 \%)$ and body composition parameters, including the L3-skeletal muscle index (PANCO-9a: $43.0 \mathrm{~cm}^{2} / \mathrm{m}^{2}$ vs PANCO-12a: $36.1 \mathrm{~cm}^{2} / \mathrm{m}^{2}$ ) and the L3-visceral adipose tissue index (PANCO-9a: $61.9 \mathrm{~cm}^{2} / \mathrm{m}^{2}$ vs PANCO-12a: $25.8 \mathrm{~cm}^{2} / \mathrm{m}^{2}$ ), which are representative for whole body muscle mass and fat mass, respectively (26).

We first investigated the potential of tumor organoid-derived CM to induce NF-kB activation in $\mathrm{C} 2 \mathrm{C} 12$ myotubes. Mature $\mathrm{C} 2 \mathrm{C} 12$ myotubes were exposed to pancreatic tumor organoid CM for $4 \mathrm{~h}$ (Fig. 2A). Whereas TNF- $\alpha$ administration caused a 6.9-fold $(p<0.001)$ increase of NF-KB activity, CM from PANCO-12a resulted in a mere 1.8 -fold $(p=0.001)$ increase of NF-KB activity, and PANCO-9a-derived CM did not induce any appreciable NF-KB activation.

We next assessed whether tumor organoid-derived $C M$ induced upregulation of E3 ligases in mature $\mathrm{C} 2 \mathrm{C} 12$ myotubes. Whereas HBSS resulted in significantly increased mRNA

A

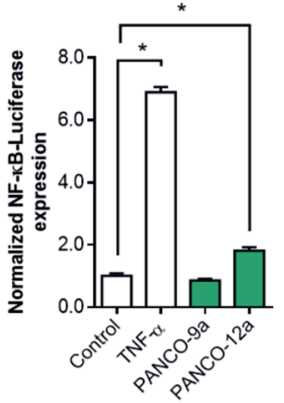

B

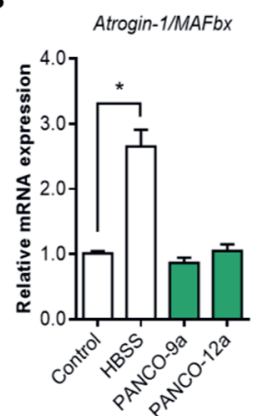

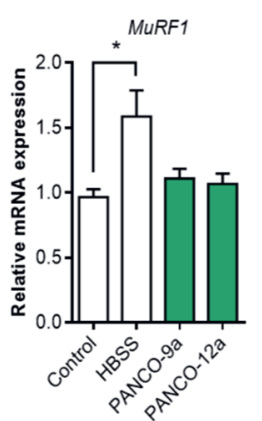

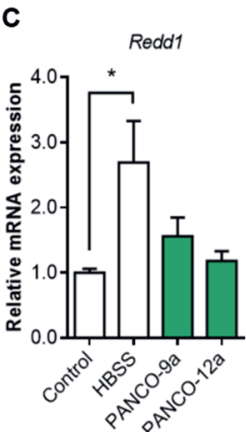

Figure 2: Pancreatic tumor organoid-derived factors do not induce $\mathrm{C2C12}$ muscle atrophy signaling. Mature $\mathrm{C} 2 \mathrm{C} 12$ myotubes were treated with CM from PANCO-9a and PANCO-12a organoid cultures. (A) After 4h, NF-KB luciferase activity luciferase was assessed. (B) mRNA expression of Atrogin$1 / M A F b x$, MURF1 and (C) Redd1 was determined after 24h. Data were normalized to CYPA, B2M, and $R P L P O$ reference genes. Relative expression levels are obtained from three independent experiments. Data are presented as mean \pm SEM. 
expression of Atrogin-1/MAFbx (2.7-fold, $\mathrm{p}=0.004$ ) and MuRF1 (1.6-fold, $\mathrm{p}=0.002$ ), no increase of either E3-ubiquitin ligase was observed in $\mathrm{C} 2 \mathrm{C} 12$ myotubes incubated with $\mathrm{CM}$ from either tumor organoid culture (Fig. 2B).

Because maintenance of skeletal muscle mass is coordinated by a balance between rates of protein synthesis and protein degradation, we also assessed the expression levels of REDD1, a repressor of protein synthesis signaling which is elevated in skeletal muscle during various atrophic conditions $(33,34)$. As expected, REDD1 levels were significantly elevated after culturing $\mathrm{C} 2 \mathrm{C} 12$ myotubes in HBSS (2.7-fold, $\mathrm{p}=0.011$ ) (Fig. $2 \mathrm{C}$ ). In contrast, incubation with tumor organoid-derived CM did not affect REDD1 expression (Fig. 2C).

Human pancreatic tumor organoid-derived factors promote myoblast fusion during differentiation

As myogenesis is an important process in the maintenance of muscle mass, we next explored the impact of tumor organoid factors on myogenesis. Impaired myogenesis and muscle regeneration have previously been observed in multiple muscle pathologies, including cancer-associated cachexia $(15,16,35-37)$. When C2C12 myoblasts were exposed to tumor organoid-derived $\mathrm{CM}$ during differentiation, pronounced disturbances in the morphology of the myotubes were observed after completion of the differentiation process (Fig. 3A). Whereas myoblasts incubated with unconditioned control medium differentiated normally into myotubes within five days, the presence of either tumor organoid CM caused a highly heterogeneous appearance of both very large myotubes and detached myotubes. To examine this impact of organoid CM on myotube morphology in more detail, we monitored myotube formation using live cell imaging (Video S1-S3). Interestingly, compared to the control, CM from both organoid cultures markedly accelerated the alignment of C2C12 myoblasts followed by strongly enhanced fusion into myotubes already after 48 hours. Strikingly, detachment of large myotubes was frequently observed in the presence of $\mathrm{CM}$, and occurred even prior to completion of myotube formation in control conditions (Fig. S1). These observations prompted us to further investigate at which level myogenesis was affected by the pancreatic tumor organoid-derived factors.

Transient increase of $\mathrm{C} 2 \mathrm{C} 12$ myoblast proliferation by pancreatic tumor organoidderived factors

We first investigated myoblast proliferation during differentiation in the presence or absence of tumor organoid CM by live cell imaging of $\mathrm{C} 2 \mathrm{C} 12$ cultures, using NucLight Red nuclear staining (Fig. 3B). In all conditions, the number of cells increased throughout the first $48 \mathrm{~h}$ of differentiation, corresponding to the cell cycle exit which is required for terminal differentiation (Fig. 3C-D). However, CM from both organoid cultures significantly enhanced proliferation within the first $48 \mathrm{~h}$ in comparison to the unconditioned control medium (Fig. $3 C)$. The enhancement was intermediate to that observed after stimulation by IGF-1, a highly potent inducer of myoblast proliferation and myogenesis. Remarkably, transcript levels of cyclin D1 (CCND1), a gene with a cell cycle-dependent expression pattern, was 
significantly decreased in $\mathrm{C} 2 \mathrm{C} 12$ myoblasts differentiated with CM from PANCO-9a (-1.8fold, $\mathrm{p}<0.001)$ and PANCO-12a $(-1.7$-fold, $\mathrm{p}<0.001)$ compared to control DM after $48 \mathrm{~h}$ (Fig. $3 E)$. This suggests that fewer cycling myoblasts remained, and that the proliferative effect of PANCO-CM is transient in nature.

Increased expression of muscle-specific genes and myogenic fusion induced by pancreatic tumor organoid-derived factors

To investigate whether myogenic differentiation was affected by tumor organoid-derived factors, we first validated the expression of key-myogenic regulators, muscle specific genes, and genes involved in excitation-contraction (EC)-coupling in muscle (Fig. 4A and Fig. S2), confirming the suitability of this system to monitor myogenesis in vitro. Next, $\mathrm{C} 2 \mathrm{C} 12$ myoblasts were exposed to tumor-organoid CM from PANCO-9a and PANCO-12a during differentiation, and expression of transcription factor paired-box 7 (Pax-7), a satellite cell marker which has been postulated to be a critical mediator of asymmetric cell division of these muscle progenitor cells (38), was monitored. After $48 \mathrm{~h}$ of differentiation, PAX7 was significantly downregulated (PANCO-9a: -2.1-fold, $\mathrm{p}<0.001$ and PANCO-12a -2.0-fold, $p<0.001$ ) compared to the control (Fig. 4B). In addition, we observed significantly reduced mRNA levels of myogenic factor 5 (MYF5) (PANCO-9a: -2.1-fold, $p<0.001$ and PANCO-12a: 1.8 -fold, $\mathrm{p}<0.001$ ). Expression of myogenic differentiation 1 (MYOD) remained unaltered at this time point (Fig.4C). Although the increase in $\mathrm{C} 2 \mathrm{C} 12$ myogenin (MYOG) expression after exposure to tumor organoid CM was not significant, the markedly elevated transcript levels of muscle creatine kinase (MCK) after 48 hours (PANCO-9a: 3.3-fold, $p=0.005$ and PANCO12a: 2.7-fold, $p=0.032$ ) indicated that myogenic differentiation was enhanced (Fig. $4 \mathrm{C}$ ). In line, mRNA levels of myomaker ( $M Y M K$ ) were significantly increased (PANCO-9a: 2.4-fold, $\mathrm{p}=0.001$ and PANCO-12a: 2.2 -fold, $p=0.004$ ) and corresponded to the strong increase in the number of nuclei residing inside myotubes (Fig. 4C). Collectively, these data indicate that pancreatic tumor organoid-derived factors accelerate the myogenic differentiation process. 
A

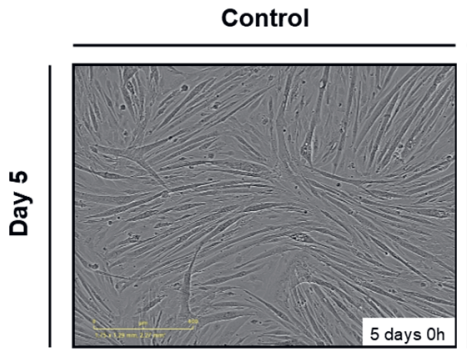

CM PANCO-9a
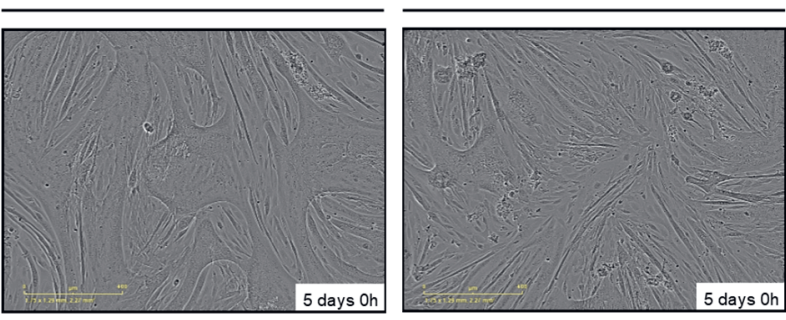

B

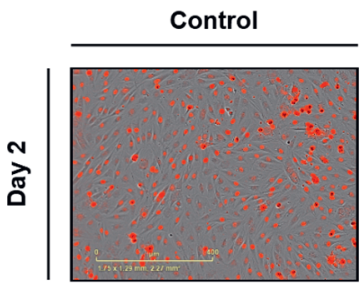

10 nM IGF-1

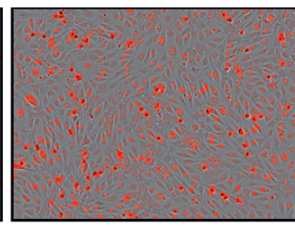

CM PANCO-9a

CM PANCO-12a
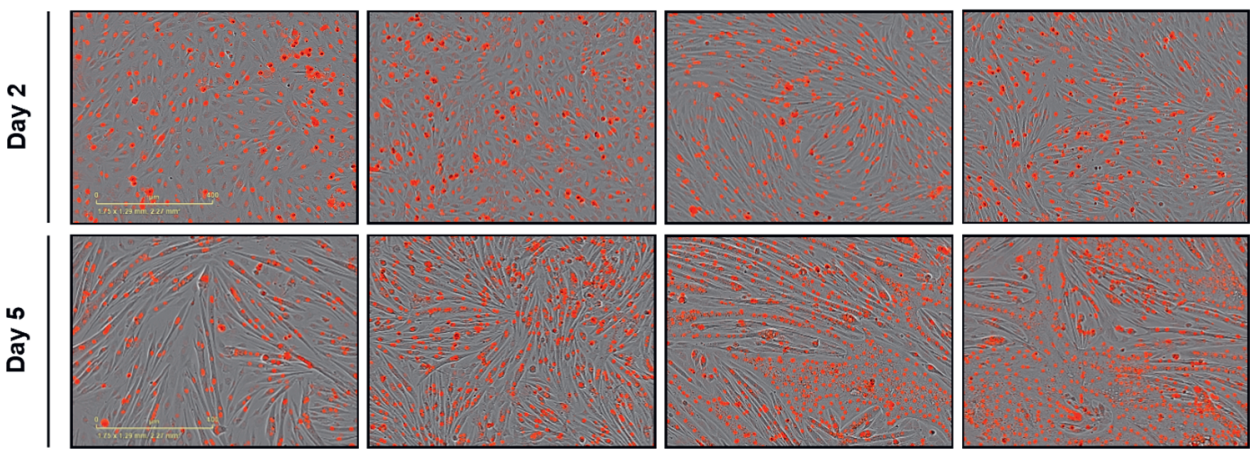

C

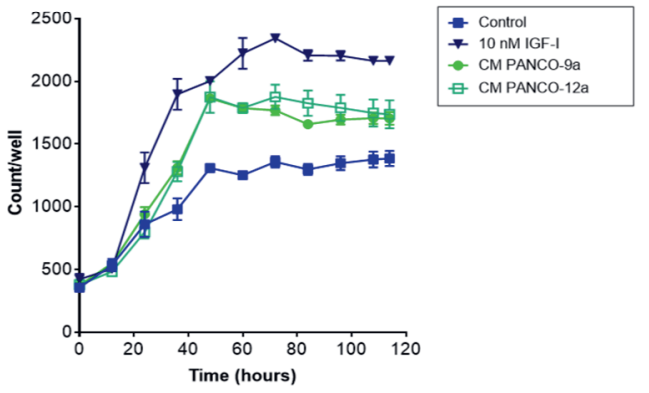

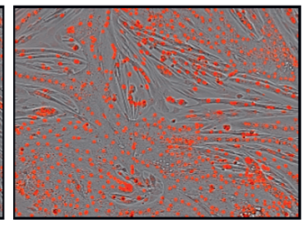

E

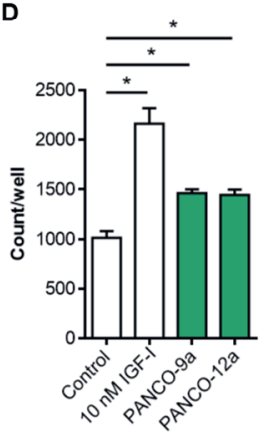

CCND1

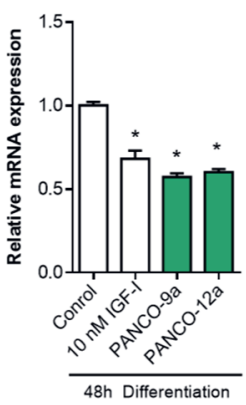

Figure 3: Tumor organoid-derived $\mathrm{CM}$ transiently stimulates proliferation and increases fusion of myoblasts during differentiation. (A) Representative phase-contrast images of $\mathrm{C} 2 \mathrm{C} 12$ myoblasts differentiated for 5 days in DM control medium (DM, 50\% (v/v) DMEM/F12) or DM containing 50\% (v/v) organoid CM. Scale bar $=400 \mu \mathrm{m}$. (B) C2C12 myoblasts were differentiated in DM control medium (DM, 50\% (v/v) DMEM/F12), DM containing $10 \mathrm{nM} \mathrm{IGF-1,} \mathrm{or} \mathrm{DM} \mathrm{containing} \mathrm{50 \%} \mathrm{(v/v)}$ organoid CM. NucLight Rapid Red was used to stain nuclei. Representative phase-contrast images overlaid with red-fluorescence images are presented. Scale bar $=400 \mu \mathrm{m}$. (C) The number of red stained nuclei on each individual image ( $12 \mathrm{~h}$ time interval) was quantified and plotted against time. (D) Bar graph showing the number of nuclei after $48 \mathrm{~h}$ of differentiation. Nuclei counts were obtained from three independent experiments. Data are presented as mean \pm SEM. (E) mRNA expression of CCND1 was determined after $48 \mathrm{~h}$. Data was normalized to CYPA, B2M, and RPLPO reference genes. Relative expression levels are obtained from three independent experiments. Data are presented as mean \pm SEM. 
Tumor organoid-derived factors increase the expression of glycolytic fast-twitch fiber MyHC-IIB

Recent evidence indicates that disturbances of EC-coupling may represent a common underlying phenomenon in the pathophysiology of many skeletal muscular disorders including cachexia and sarcopenia $(39,40)$. In particular, downregulation of $\mathrm{Ca}^{2+}$ fluxregulating signaling channels impairs muscle function. However, in line with the enhanced myogenesis, we also observed significantly increased expression of $\mathrm{Ca}^{2+}$ flux regulating signaling channels (RYR1, SERCA2, DHPR, and CACNB1) in differentiating C2C12 myoblasts that were stimulated with CM from either tumor organoid (Fig. 4D).

Finally, we investigated whether organoid-derived $\mathrm{CM}$ affected the expression of genes encoding contractile proteins, focusing on myosin heavy chain (MyHC) isoforms. Compared to the control, mRNA expression levels of the glycolytic fast-twitch myofiber MyHC-IIB (MYH4) was significantly increased by CM from PANCO-9a (2.5-fold, $\mathrm{p}=0.04$ ) and PANCO-12a (3.1-fold, $\mathrm{p}=0.006$ ) after $72 \mathrm{~h}$ of differentiation (Fig. 4E). Altogether, these data imply that pancreatic tumor organoid-derived factors accelerate myogenesis, which is accompanied by a shift towards a myosin expression pattern that is frequently observed in cachexia. 
A
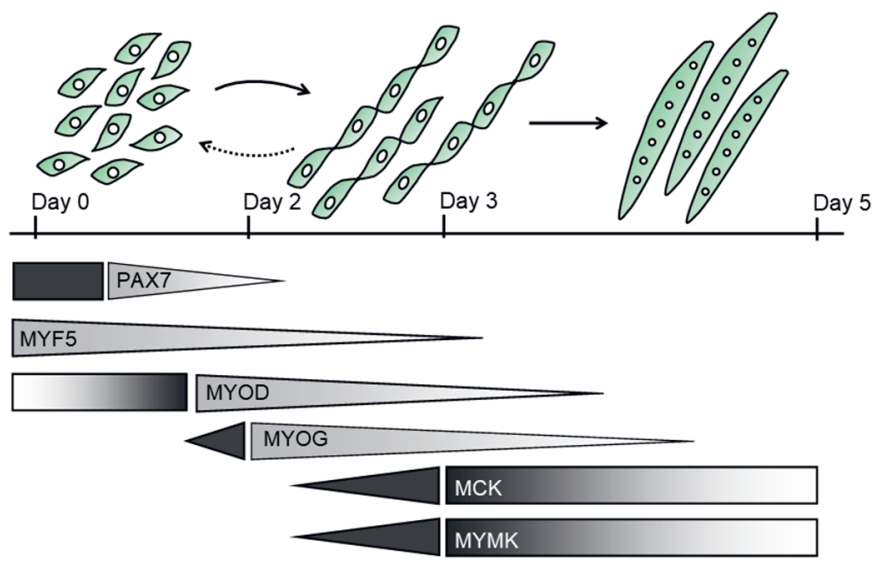

B

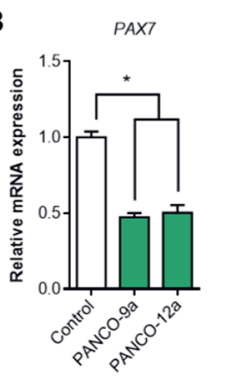

C MYF5
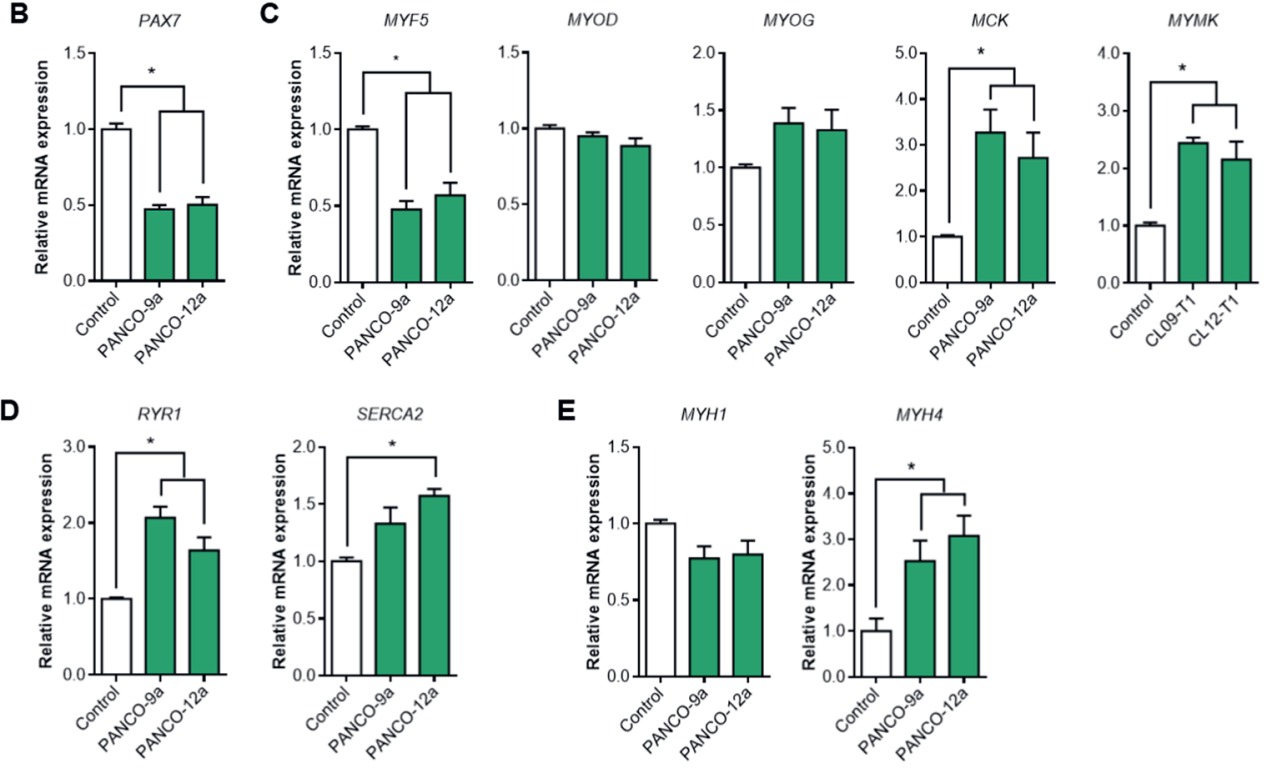

E
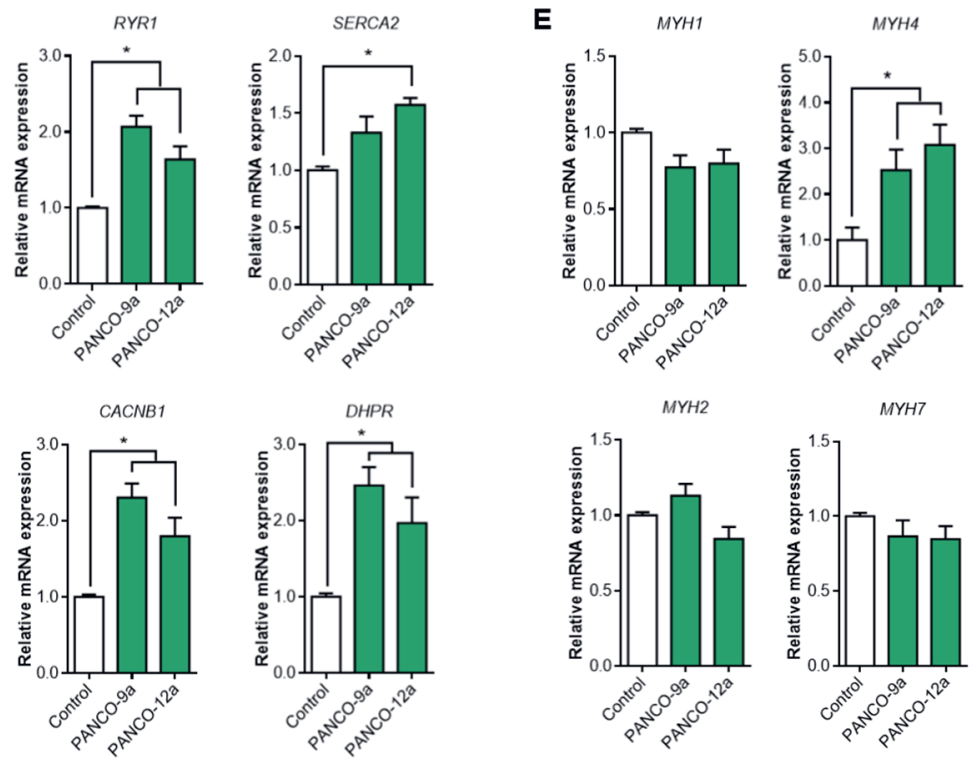
< Previous page. Figure 4: Enhanced myogenic differentiation induced by tumor organoid-derived $\mathrm{CM}$ is accompanied by increased MyHC-Ilb expression and suppression of self-renewal markers. (A) Graphical representation of the myogenic process. Myoblasts have the ability to proliferate, selfrenew, differentiate, and fuse into myotubes. During proliferation, myoblasts express the paired-box 7 (PAX7) transcription factor. Upon myogenic commitment, a decrease in Pax7 expression results in cell cycle arrest, which is accompanied by increased expression of myogenic factor 5 (MYF5) and myoblast determination (MYOD) transcription factors. Myogenin (MYOG) is a transcription factor that is highly expressed during the fusion of myoblasts into myotubes. This protein results in the transcription of genes required for the fusion of myoblasts into myotubes, including myomaker (MYMK). mRNA expression of (B) satellite cell marker PAX7, (C) key-myogenic regulators and muscle specific genes (MYF5, MYOD, MYOG, MCK, MYMK), and (D) $\mathrm{Ca}^{2+}$ flux regulating signaling channels (RYR1, SERCA2, CACNB1, DHPR) were determined after 48h. (E) Myosin heavy chain isoforms (MYH1, $\mathrm{MYH} 2, \mathrm{MYH} 4, \mathrm{MYH7}$ ) were determined after $72 \mathrm{~h}$. Data were normalized to CYPA, B2M, and RPLPO reference genes. Relative expression levels are obtained from three independent experiments. Data are presented as mean \pm SEM. CACNB1, calcium voltage-gated channel auxiliary subunit beta1; DHPR, dihydropteridine reductase; $M C K$, muscle creatine kinase; $M Y H 1$, myosin heavy chain 1, MyHC-IIx; MYH2, myosin heavy chain 2, MyHC-Ila; MYH4, myosin heavy chain 4, MyHC-Illb; MYH7, myosin heavy chain 7, MyHC-I; RYR1, ryanodine receptor 1; SERCA2, Sarcoplasmic/endoplasmic reticulum calcium ATPase 2. 


\section{Discussion}

The loss of skeletal muscle is a clinically significant feature of cancer cachexia and is assumed to result from direct and/or indirect actions of tumor-derived factors. At present, there is a lack of adequate pre-clinical models that can be used to assess the direct effect of tumor-derived factors of cachectic cancer patients on muscle wasting. In the current study, we first showed that the factors released by four different conventional human pancreatic cancer cell lines cultured in 2D had minimal and inconsistent effects on atrophy signaling pathways in skeletal muscle cells. Similarly, CM from pancreatic tumor organoids obtained from either a cachectic or non-cachectic patient did not induce muscle atrophy signaling in mature $\mathrm{C} 2 \mathrm{C} 12$ myotubes. Unexpectedly, however, pancreatic tumor organoidderived factors transiently increased proliferation of $\mathrm{C} 2 \mathrm{C} 12$ myoblasts and accelerated their alignment and fusion. This enhanced myogenesis was accompanied by altered expression of factors governing muscle cell differentiation and a selective increase in MyHC-IIB, which is preferentially expressed in atrophy-sensitive glycolytic myofibers. Interestingly, these tumor organoid CM effects were not associated with the cachexia phenotype of the donor patients.

The loss of skeletal muscle tissue in cachexia has been suggested to result from tumorderived factors that activate the ubiquitin-proteasome pathway to target major contractile skeletal muscle proteins for degradation. However, we found that the secretome of most conventionally cultured human pancreatic cancer cell lines only minimally affected the expression of the E3-ubiquitin ligases Atrogin-1/MAFbx and MuRF1 in mature C2C12 myotubes. Similar results were obtained in our pancreatic tumor organoid experiments. These data are not in line with previous in vitro studies that showed tumor-induced muscle atrophy via activation of the ubiquitin proteasome pathway. For example, treatment of C2C12 mouse skeletal muscle myotubes with C26- and LLC-derived factors has been reported to induce significant upregulation of the E3-ubiquitin ligase Atrogin1/MAFbx, loss of myosin heavy chain proteins, and reduction of myofiber size $(41,42)$. However, in line with our results, Talbert et al. did not detect differential expression of these genes in muscle biopsies from cachectic versus non-cachectic pancreatic cancer patients (18). Similar observations were made in muscles from cachectic lung cancer patients: compared to healthy subjects, cachectic lung cancer patients showed no differences in E3 ubiquitin ligase expression despite reduced muscle mass, muscle fiber atrophy, and decreased quadriceps strength (43). Furthermore, we did not observe noticeable morphological muscle cell atrophy after exposure to tumor cell-derived or tumor organoid-derived factors. Although this contrasts with findings of other studies $(8,41,42)$, our data are in line with a recent study of Guigni et al. in which the effect of murine and human lung tumor secreted factors on skeletal muscle was investigated (44). Their report revealed that in fully matured C2C12 myotubes, myotube size and myosin heavy chain content were not affected by tumorderived factors. Altogether, these data do not support activation of the proteasome by E3 Ub-ligases and subsequent myofiber atrophy as a direct effect of pancreas tumor derived 
factors. Instead, other processes in muscle cells may be affected by tumor-derived factors and contribute to the loss of muscle mass in human cancer-induced cachexia.

One of these processes is myogenesis, which involves activation and asymmetric cell division of satellite cells, followed by either repletion of this pool of quiescent muscle precursor cells, or by proliferation of daughter myoblasts and subsequent myogenic differentiation and fusion into myotubes (45). Counterintuitively, pancreatic tumor-derived factors provoked the development of both enlarged and disrupted myotubes after five days of differentiation. Live cell imaging-based monitoring of morphological changes of $\mathrm{C} 2 \mathrm{C} 12$ myoblasts during differentiation revealed that the disrupted myotube morphology resulted from early detachment of large myotubes which was followed by formation of new myotubes. This was preceded by accelerated alignment of $\mathrm{C} 2 \mathrm{C} 12$ myoblasts and early muscle specific gene expression. Importantly, the transient increase in myoblast proliferation in the presence of tumor-derived factors that we observed is in line with previous reports on increases in the number of proliferating muscle precursor cells in atrophying muscle $(15,16)$. Similar observations have previously been made in cancer cachexia studies. For example, increased numbers of Pax-7 expressing cells have been found in the muscle of tumor-bearing animals and cachectic pancreatic cancer patients, which was interpreted as an aberrant proliferative response of muscle precursor cells $(14,36)$. While we found that myoblast proliferation initially increased in response to tumor derived factors, cyclin D1 expression was markedly reduced thereafter, in line with aberrant myoblast proliferative kinetics. In vivo, myoblast proliferation is subject to asymmetric cell division by activated muscle progenitor cells, governed by Pax-7 expression (45). Upon entering the myogenic program, myoblasts committing to terminal differentiation lose Pax7 expression, whereas Pax-7 expression is maintained by myoblasts that become quiescent to replete the satellite cell population. The reduced Pax-7 expression in myoblast cultures in response to the tumor organoid-derived factors may therefore reflect a depletion of the population of replication competent cells, referred to as 'reserve cells', which represent the in vitro equivalents of quiescent satellite cells (46). In vivo, such a depletion of muscle precursor cells will interfere with long term myogenic potential, ultimately contributing to muscle mass loss. In support of this notion, cultured muscle precursor cells isolated from cachectic tumor-bearing mice have been reported to differentiate more readily compared to those of control mice $(36,47)$. Taken together, these data imply that in response to tumor-derived factors, muscle precursor cells are directed towards myogenic commitment at the expense of the quiescent satellite cell repopulation, which may contribute to the impaired muscle mass maintenance that is observed in cachexia.

Next to the altered myogenesis, we observed increased levels of the glycolytic fast-twitch myofiber MyHC-IIB in response to tumor organoid-derived factors. This is consistent with the shift in muscle fiber type composition that has been reported in mice bearing C26 carcinomas: the type Illb myosin isoform comprised $19 \%$ of their total myosin fiber content, whereas it was not detected in control mice (48). Since it has been suggested that type 2 muscle fibers are more sensitive to atrophy-inducing stimuli, a tumor factor-driven shift 
towards more fast twitch MyHC-II expression may sensitize muscle fibers to additional catabolic triggers in cancer cachexia $(49,50)$. In line with this, wasting of muscle fibers in cancer cachexia is more pronounced in fast-twitch type II-containing muscles, such as tibialis anterior and gastrocnemius, compared to slow-twitch type I muscles, such as the soleus $(51,52)$. This implies that pancreatic tumor organoid-derived factors could promote the development of muscle fibers that are more prone to atrophy-inducing stimuli.

Interestingly, the pronounced effects of tumor organoid-derived factors on myogenesis and myosin heavy chain expression were not related to the cachexia status of the patients contributing the organoid cultures. In addition, only CM from PANCO-12a, originating from a non-cachectic patient, caused mild NF-kB activation in mature C2C12 myotubes. This may result from the relatively higher expression of known inducers of NF-KB signaling such as interleukin 1 (IL-1) and TNF- $\alpha$ by PANCO-12a organoids (26). Given these data as well as the marked impact of tumor organoid-derived factors on myogenesis, more detailed analyses of the organoid secretome to identify the factors responsible are warranted. These studies will also help to distinguish direct and indirect effects of tumor-derived factors on skeletal muscle (patho)physiology in relation to the cachexia status of the donor patient, thereby contributing to a better understanding of cachexia-inducing mechanisms.

In conclusion, we showed that pancreatic tumor organoid-derived factors alter the kinetics of myogenesis consistent with depletion of skeletal muscle precursor cells and diminished myogenic potential. Organoids therefore represent a promising new model to investigate mechanisms of muscle loss in human cancer cachexia. 


\section{References}

1. Bray F, Ferlay J, Soerjomataram I, Siegel RL, Torre LA, Jemal A. Global cancer statistics 2018: GLOBOCAN estimates of incidence and mortality worldwide for 36 cancers in 185 countries. CA Cancer J Clin. 2018;68(6):394-424.

2. Kleeff J, Korc M, Apte M, La Vecchia C, Johnson CD, Biankin AV, et al. Pancreatic cancer. Nat Rev Dis Primers. 2016;2:16022.

3. Baracos VE, Martin L, Korc M, Guttridge DC, Fearon KCH. Cancer-associated cachexia. Nat Rev Dis Primers. 2018;4:17105.

4. Fearon K, Strasser F, Anker SD, Bosaeus I, Bruera E, Fainsinger RL, et al. Definition and classification of cancer cachexia: an international consensus. Lancet Oncol. 2011;12(5):48995.

5. Callaway CS, Delitto AE, Patel R, Nosacka RL, D'Lugos AC, Delitto D, et al. IL-8 Released from Human Pancreatic Cancer and Tumor-Associated Stromal Cells Signals through a CXCR2ERK1/2 Axis to Induce Muscle Atrophy. Cancers (Basel). 2019;11(12).

6. Delitto D, Judge SM, Delitto AE, Nosacka RL, Rocha FG, DiVita BB, et al. Human pancreatic cancer xenografts recapitulate key aspects of cancer cachexia. Oncotarget. 2017;8(1):117789.

7. Henderson SE, Makhijani N, Mace TA. Pancreatic Cancer-Induced Cachexia and Relevant Mouse Models. Pancreas. 2018;47(8):937-45.

8. Jackman RW, Floro J, Yoshimine R, Zitin B, Eiampikul M, El-Jack K, et al. Continuous Release of Tumor-Derived Factors Improves the Modeling of Cachexia in Muscle Cell Culture. Front Physiol. 2017;8:738.

9. Kandarian SC, Nosacka RL, Delitto AE, Judge AR, Judge SM, Ganey JD, et al. Tumour-derived leukaemia inhibitory factor is a major driver of cancer cachexia and morbidity in C26 tumourbearing mice. J Cachexia Sarcopenia Muscle. 2018;9(6):1109-20.

10. Zhang G, Liu Z, Ding H, Zhou Y, Doan HA, Sin KWT, et al. Tumor induces muscle wasting in mice through releasing extracellular Hsp70 and Hsp90. Nat Commun. 2017;8(1):589.

11. Wyke SM, Russell ST, Tisdale MJ. Induction of proteasome expression in skeletal muscle is attenuated by inhibitors of NF-kappaB activation. Br J Cancer. 2004;91(9):1742-50.

12. Acharyya S, Butchbach ME, Sahenk Z, Wang H, Saji M, Carathers M, et al. Dystrophin glycoprotein complex dysfunction: a regulatory link between muscular dystrophy and cancer cachexia. Cancer Cell. 2005;8(5):421-32.

13. Cai D, Frantz JD, Tawa NE, Jr., Melendez PA, Oh BC, Lidov HG, et al. IKKbeta/NF-kappaB activation causes severe muscle wasting in mice. Cell. 2004;119(2):285-98.

14. Talbert EE, Metzger GA, He WA, Guttridge DC. Modeling human cancer cachexia in colon 26 tumor-bearing adult mice. J Cachexia Sarcopenia Muscle. 2014;5(4):321-8.

15. Inaba S, Hinohara A, Tachibana M, Tsujikawa K, Fukada SI. Muscle regeneration is disrupted by cancer cachexia without loss of muscle stem cell potential. PLoS One. 2018;13(10):e0205467.

16. Penna F, Costamagna D, Fanzani A, Bonelli G, Baccino FM, Costelli P. Muscle wasting and impaired myogenesis in tumor bearing mice are prevented by ERK inhibition. PLoS One. 2010;5(10):e13604.

17. Wang G, Biswas AK, Ma W, Kandpal M, Coker C, Grandgenett PM, et al. Metastatic cancers promote cachexia through ZIP14 upregulation in skeletal muscle. Nat Med. 2018;24(6):77081.

18. Talbert EE, Cuitino MC, Ladner KJ, Rajasekerea PV, Siebert M, Shakya R, et al. Modeling Human Cancer-induced Cachexia. Cell Rep. 2019;28(6):1612-22 e4. 
19. Shukla SK, Gebregiworgis T, Purohit V, Chaika NV, Gunda V, Radhakrishnan P, et al. Metabolic reprogramming induced by ketone bodies diminishes pancreatic cancer cachexia. Cancer Metab. 2014;2:18.

20. Togashi $Y$, Kogita A, Sakamoto H, Hayashi H, Terashima M, de Velasco MA, et al. Activin signal promotes cancer progression and is involved in cachexia in a subset of pancreatic cancer. Cancer Lett. 2015;356(2 Pt B):819-27.

21. Baker BM, Chen CS. Deconstructing the third dimension: how 3D culture microenvironments alter cellular cues. J Cell Sci. 2012;125(Pt 13):3015-24.

22. Ben-David U, Siranosian B, Ha G, Tang H, Oren Y, Hinohara K, et al. Genetic and transcriptional evolution alters cancer cell line drug response. Nature. 2018;560(7718):32530.

23. Boj SF, Hwang Cl, Baker LA, Chio, II, Engle DD, Corbo V, et al. Organoid models of human and mouse ductal pancreatic cancer. Cell. 2015;160(1-2):324-38.

24. Clevers H. Modeling Development and Disease with Organoids. Cell. 2016;165(7):1586-97.

25. Lancaster MA, Huch M. Disease modelling in human organoids. Dis Model Mech. 2019;12(7).

26. Vaes RDW, van Dijk DPJ, Welbers TTJ, Blok MJ, Aberle MR, Heij L, et al. Generation and initial characterization of novel tumour organoid models to study human pancreatic cancerinduced cachexia. J Cachexia Sarcopenia Muscle. 2020.

27. Lieber M, Mazzetta J, Nelson-Rees W, Kaplan M, Todaro G. Establishment of a continuous tumor-cell line (panc-1) from a human carcinoma of the exocrine pancreas. Int $J$ Cancer. 1975;15(5):741-7.

28. Kobari M, Matsuno S, Sato T, Kan M, Tachibana T. Establishment of a human pancreatic cancer cell line and detection of pancreatic cancer associated antigen. Tohoku J Exp Med. 1984;143(1):33-46.

29. Kimura $Y$, Kobari M, Yusa T, Sunamura M, Kimura M, Shimamura H, et al. Establishment of an experimental liver metastasis model by intraportal injection of a newly derived human pancreatic cancer cell line (KLM-1). Int J Pancreatol. 1996;20(1):43-50.

30. Langen RC, Schols AM, Kelders MC, Wouters EF, Janssen-Heininger YM. Inflammatory cytokines inhibit myogenic differentiation through activation of nuclear factor-kappaB. FASEB J. 2001;15(7):1169-80.

31. Ruijter JM, Ramakers C, Hoogaars WM, Karlen Y, Bakker O, van den Hoff MJ, et al. Amplification efficiency: linking baseline and bias in the analysis of quantitative PCR data. Nucleic Acids Res. 2009;37(6):e45.

32. Bodine SC, Baehr LM. Skeletal muscle atrophy and the E3 ubiquitin ligases MuRF1 and MAFbx/atrogin-1. Am J Physiol Endocrinol Metab. 2014;307(6):E469-84.

33. Britto FA, Cortade F, Belloum Y, Blaquiere M, Gallot YS, Docquier A, et al. Glucocorticoiddependent REDD1 expression reduces muscle metabolism to enable adaptation under energetic stress. BMC Biol. 2018;16(1):65.

34. Gordon BS, Williamson DL, Lang CH, Jefferson LS, Kimball SR. Nutrient-induced stimulation of protein synthesis in mouse skeletal muscle is limited by the mTORC1 repressor REDD1. $J$ Nutr. 2015;145(4):708-13.

35. Arneson PC, Doles JD. Impaired Muscle Regeneration in Cancer-Associated Cachexia. Trends Cancer. 2019;5(10):579-82.

36. He WA, Berardi E, Cardillo VM, Acharyya S, Aulino P, Thomas-Ahner J, et al. NF-kappaBmediated Pax7 dysregulation in the muscle microenvironment promotes cancer cachexia. $J$ Clin Invest. 2013;123(11):4821-35.

37. Hogan KA, Cho DS, Arneson PC, Samani A, Palines P, Yang Y, et al. Tumor-derived cytokines impair myogenesis and alter the skeletal muscle immune microenvironment. Cytokine. 2018;107:9-17.

38. Kuang S, Kuroda K, Le Grand F, Rudnicki MA. Asymmetric self-renewal and commitment of satellite stem cells in muscle. Cell. 2007;129(5):999-1010. 
39. Agrawal A, Suryakumar $\mathrm{G}$, Rathor R. Role of defective $\mathrm{Ca}(2+)$ signaling in skeletal muscle weakness: Pharmacological implications. J Cell Commun Signal. 2018;12(4):645-59.

40. Fontes-Oliveira CC, Busquets S, Fuster G, Ametller E, Figueras M, Olivan M, et al. A differential pattern of gene expression in skeletal muscle of tumor-bearing rats reveals dysregulation of excitation-contraction coupling together with additional muscle alterations. Muscle Nerve. 2014;49(2):233-48.

41. Zhang G, Jin B, Li YP. C/EBPbeta mediates tumour-induced ubiquitin ligase atrogin1/MAFbx upregulation and muscle wasting. EMBO J. 2011;30(20):4323-35.

42. Zhang G, Liu Z, Ding H, Miao H, Garcia JM, Li YP. Toll-like receptor 4 mediates Lewis lung carcinoma-induced muscle wasting via coordinate activation of protein degradation pathways. Sci Rep. 2017;7(1):2273.

43. Op den Kamp CM, Langen RC, Snepvangers FJ, de Theije CC, Schellekens JM, Laugs F, et al. Nuclear transcription factor kappa B activation and protein turnover adaptations in skeletal muscle of patients with progressive stages of lung cancer cachexia. Am J Clin Nutr. 2013;98(3):738-48.

44. Guigni BA, van der Velden J, Kinsey CM, Carson JA, Toth MJ. Effects of conditioned media from murine lung cancer cells and human tumor cells on cultured myotubes. Am J Physiol Endocrinol Metab. 2020;318(1):E22-E32.

45. Motohashi N, Asakura A. Muscle satellite cell heterogeneity and self-renewal. Front Cell Dev Biol. 2014;2:1.

46. Yoshida N, Yoshida S, Koishi K, Masuda K, Nabeshima Y. Cell heterogeneity upon myogenic differentiation: down-regulation of MyoD and Myf-5 generates 'reserve cells'. J Cell Sci. 1998;111 ( Pt 6):769-79.

47. Talbert EE, Guttridge DC. Impaired regeneration: A role for the muscle microenvironment in cancer cachexia. Semin Cell Dev Biol. 2016;54:82-91.

48. Diffee GM, Kalfas K, Al-Majid S, McCarthy DO. Altered expression of skeletal muscle myosin isoforms in cancer cachexia. Am J Physiol Cell Physiol. 2002;283(5):C1376-82.

49. Matsakas A, Patel K. Skeletal muscle fibre plasticity in response to selected environmental and physiological stimuli. Histol Histopathol. 2009;24(5):611-29.

50. Schiaffino S, Reggiani C. Fiber types in mammalian skeletal muscles. Physiol Rev. 2011;91(4):1447-531.

51. Acharyya S, Ladner KJ, Nelsen LL, Damrauer J, Reiser PJ, Swoap S, et al. Cancer cachexia is regulated by selective targeting of skeletal muscle gene products. J Clin Invest. 2004;114(3):370-8.

52. Mendell JR, Engel WK. The fine structure of type II muscle fiber atrophy. Neurology. 1971;21(4):358-65. 


\section{Supplemental table}

Table S1: qPCR mouse primer sequences

\begin{tabular}{|c|c|c|}
\hline Gene & Full name & Primers \\
\hline ATROGIN & $\begin{array}{l}\text { Atrogin-1 } \\
\text { /Muscle atrophy F-box protein }\end{array}$ & $\begin{array}{l}\text { FW: 5' - CAGCAGCTGAATAGCATCCAGAT - 3' } \\
\text { RV: } 5^{\prime}-\text { TCTGCATGATGTTCAGTTGTAAGC - 3' }\end{array}$ \\
\hline MURF & $\begin{array}{l}\text { Muscle-specific RING finger } \\
\text { protein } 1\end{array}$ & $\begin{array}{l}\text { FW: 5' - CTTCCTCTCAAGTGCCAAGCA - 3' } \\
\text { RV: 5' - GTGTTCTAAGTCCAGAGTAAAGTAGTCCAT - 3' }\end{array}$ \\
\hline CCND1 & Cyclin D1 & $\begin{array}{l}\text { FW: 5' - CATTCCCTTGACTGCCGAGAAGTT - 3' } \\
\text { RV: 5' - TTGTTCACCAGAAGCAGTTCCATTT - 3' }\end{array}$ \\
\hline PCNA & Proliferating cell nuclear antigen & $\begin{array}{l}\text { FW: 5' - CCAAATCAAGAGAAAGTTTCAGACTATGA - 3' } \\
\text { RV: 5' - TCACCCGACGGCATCTTTATT - 3' }\end{array}$ \\
\hline PAX7 & Paired box 7 & $\begin{array}{l}\text { FW: 5' - ACCAGTACAGCCAGTATGGCCA - 3' } \\
\text { RV: 5' - GTGTTCCCCAAGCTTCATACGG - 3' }\end{array}$ \\
\hline MYF5 & Myogenic factor 5 & $\begin{array}{l}\text { FW: 5' - GAACAGCAGCTTTGACAGCAT - 3' } \\
\text { RV: 5' - AATGCTGGACAAGCATCCAA - 3' }\end{array}$ \\
\hline MYOD & Myogenic differentiation 1 & $\begin{array}{l}\text { FW: 5' - GGCAGAATGGCTACGACACCG - 3' } \\
\text { RV: 5' - CTTCCCTGGCCTGGACTCGC - 3' }\end{array}$ \\
\hline MYOG & Myogenin & $\begin{array}{l}\text { FW: 5' - CCCATGGTGCCCAGTGAA - 3' } \\
\text { RV: } 5^{\prime}-\text { GCAGATTGTGGGCGTCTGTA - } 3^{\prime}\end{array}$ \\
\hline MYMK & $\begin{array}{l}\text { Myomaker, myoblast fusion } \\
\text { factor }\end{array}$ & $\begin{array}{l}\text { FW: 5' - CTGGCCGACTTTGATGAACC - 3' } \\
\text { RV: 5' - TGCTCTTGTCGGGGTACAGG - 3' }\end{array}$ \\
\hline MCK & Muscle creatine kinase & $\begin{array}{l}\text { FW: 5' - AGGTTTTCCGCCGCTTCT - 3' } \\
\text { RV: 5' - CGGTGCCCAGGTTGGA - 3' }\end{array}$ \\
\hline RYR1 & Ryanodine receptor 1 & $\begin{array}{l}\text { FW: 5' - CGCATCGAAATCATGGGCGC - 3' } \\
\text { RV: } 5^{\prime}-\text { TGAACTGGCGCTTGGACTCC - 3' }\end{array}$ \\
\hline SERCA2 & $\begin{array}{l}\text { Sarcoplasmic/endoplasmic } \\
\text { reticulum calcium ATPase } 2\end{array}$ & $\begin{array}{l}\text { FW: 5' - TCTCACGTGCCTGGTGGAGA - 3' } \\
\text { RV: 5' - TGAACTCCTTCTTCATCAGCTGCT - 3' }\end{array}$ \\
\hline CACNB1 & $\begin{array}{l}\text { Calcium voltage-gated channel } \\
\text { auxiliary subunit beta } 1\end{array}$ & $\begin{array}{l}\text { FW: 5' - CGCTGGCCCCCATCATTGTT - 3' } \\
\text { RV: 5' - CTCCGAGGCTGCTATTTGGACA - 3' }\end{array}$ \\
\hline$D H P R$ & Dihydropteridine reductase & $\begin{array}{l}\text { FW: 5' - ACGCCAATGCCAATGTTGCC - 3' } \\
\text { RV: 5' - GCATGTCCGCCTCTCCCAAG - 3' }\end{array}$ \\
\hline MYH1 & Myosin heavy chain 1, MyHC-IIx & $\begin{array}{l}\text { FW: 5' - CCTAGCCAAAGCCGTCTATGAG - 3' } \\
\text { RV: 5' - GGTGGTTGAAGAACTGTTGCAGTT - 3' }\end{array}$ \\
\hline $\mathrm{MYH2}$ & Myosin heavy chain 2, MyHC-Ila & $\begin{array}{l}\text { FW: 5' - GCCCCGCCCCACATC - 3' } \\
\text { RV: } 5^{\prime}-\text { GGATTGACTGATTCTCCCTGTCTGT - 3' }\end{array}$ \\
\hline MYH4 & Myosin heavy chain 4, MyHC-IIb & $\begin{array}{l}\text { FW: 5' - AGGGCGGCGGTGGAA - 3' } \\
\text { RV: 5' - TGGGAATGAGGCATCTGACAA - 3' }\end{array}$ \\
\hline
\end{tabular}




\begin{tabular}{|c|c|c|}
\hline MYH7 & Myosin heavy chain 7, MyHC-I & $\begin{array}{l}\text { FW: } 5^{\prime}-\text { CAGATCGGGAGAATCAGTCCAT - 3' } \\
\text { RV: } 5^{\prime} \text { - AGCAAAATATTGGATGACCCTCTTA - 3' }\end{array}$ \\
\hline CYPA & Cyclophilin A & $\begin{array}{l}\text { FW: 5' - TTCCTCCTTTCACAGAATTATTCCA - 3' } \\
\text { RV: } 5^{\prime} \text { - CCGCCAGTGCCATTATGG - 3' }\end{array}$ \\
\hline$B 2 M$ & Beta-2-microglobulin & $\begin{array}{l}\text { FW: 5' - CTTTCTGGTGCTTGTCTCACTGA - 3' } \\
\text { RV: 5' - GTATGTTCGGCTTCCCATTCTC - 3' }\end{array}$ \\
\hline$R P L P O$ & 605 Acidic ribosomal protein $\mathrm{PO}$ & $\begin{array}{l}\text { FW: 5' - GGACCCGAGAAGACCTCCTT - 3' } \\
\text { RV: } 5^{\prime}-\text { GCACATCACTCAGAATTTCAATGG - 3' }\end{array}$ \\
\hline
\end{tabular}




\section{Supplemental figures}
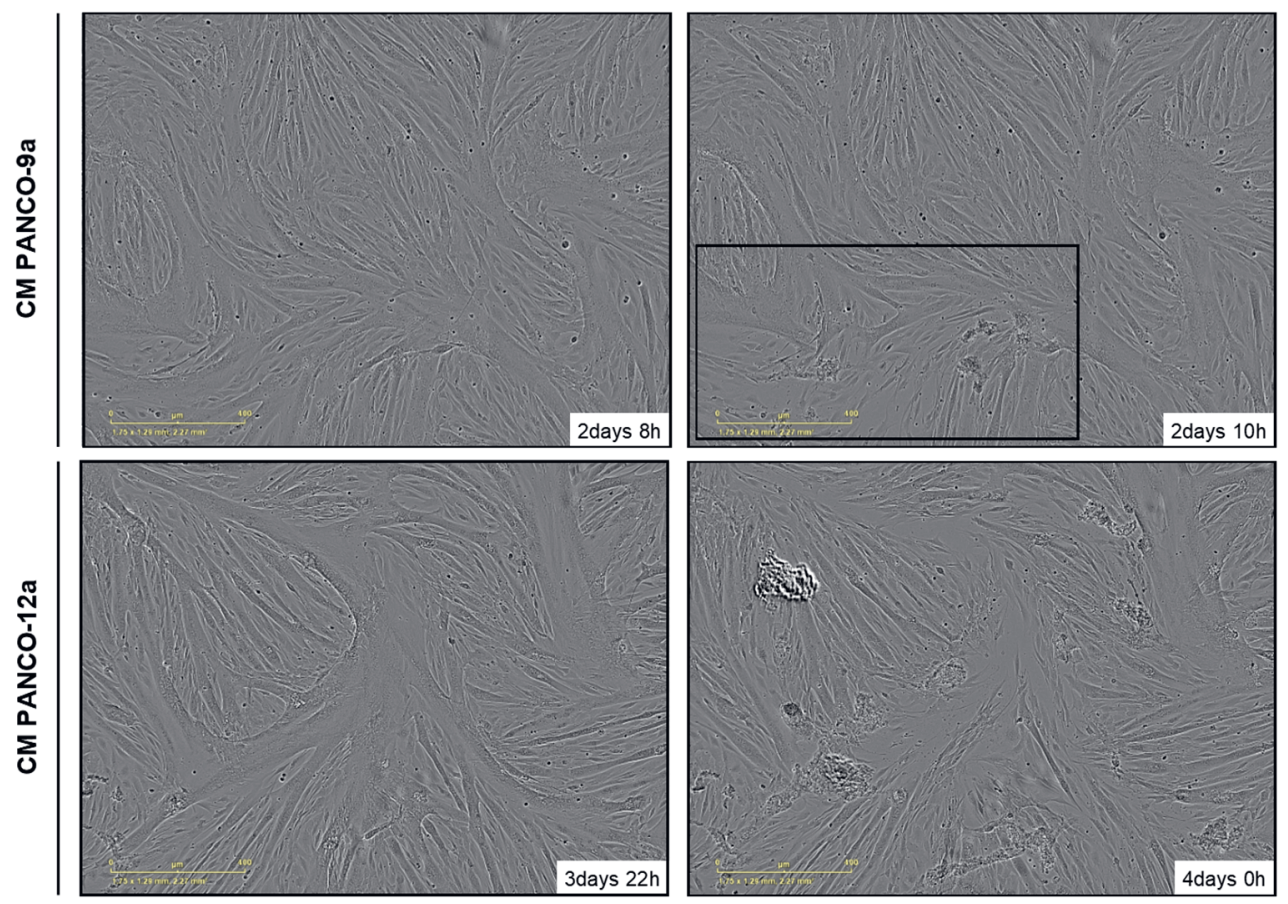

Figure S1: Tumor organoid-derived $\mathrm{CM}$ induces morphological disturbances during the differentiation of myoblasts into myotubes. $\mathrm{C} 2 \mathrm{C} 12$ myoblasts were differentiated for 5 days in DM containing 50\% (v/v) organoid CM (PANCO-9a and PANCO-12a). Sequential images ( $2 \mathrm{~h}$ time interval) show the detachment of large myotubes during differentiation. Scale bar $=400 \mu \mathrm{m}$. 

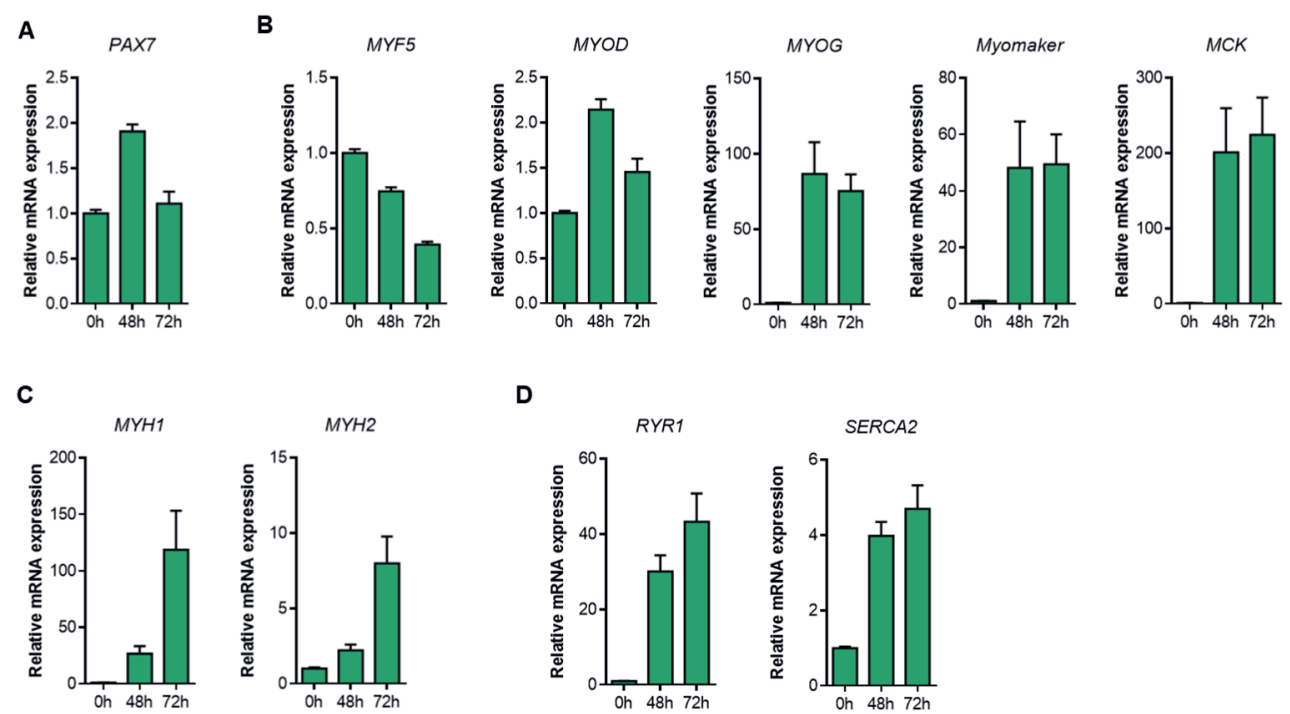

D
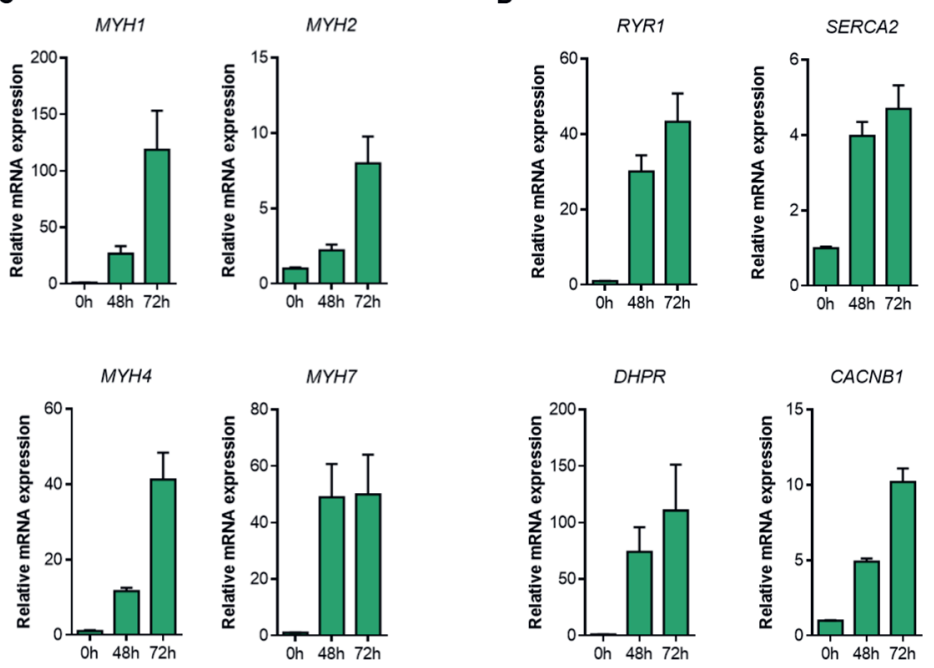

Figure S2: Gene expression of myogenic markers during myogenesis. C2C12 myoblasts were differentiated for 3 days in DM and cell lysates were collected at $0 \mathrm{~h}, 48 \mathrm{~h}$, and $72 \mathrm{~h}$ after differentiation was initiated. mRNA expression of (A) PAX7, (B) key-myogenic regulators and muscle-specific genes (MYF5, MYOD, MYOG, MYMK, MCK), (C) genes involved in excitation-contraction (EC)-coupling in muscle (RYR1, SERCA2, DHPR, CACNB1), and (D) myosin heavy chain isoforms (MYH1, MYH2, MYH4, MYH7) were determined. Data were normalized to CYPA, B2M, and RPLPO reference genes. Relative expression levels are obtained from three independent experiments. Data are presented as mean \pm SEM. 


\section{Supplemental videos}

Video S1: Myogenic differentiation - Control. C2C12 myoblasts were differentiated for 5 days in DM control medium (DM, 50\% (v/v) DMEM/F12). Scale bar $=400 \mu \mathrm{m}$.

Video S2: Myogenic differentiation - PANCO-9a. C2C12 myoblasts were differentiated for 5 days in DM containing 50\% (v/v) PANCO-9a-derived CM. Scale bar $=400 \mu \mathrm{m}$.

Video S3: Myogenic differentiation - PANCO-12a. C2C12 myoblasts were differentiated for 5 days in DM containing 50\% (v/v) PANCO-12a-derived CM. Scale bar $=400 \mu \mathrm{m}$. 


\section{CHAPTER 4}

\section{A novel human cell culture model to study visceral smooth muscle phenotypic modulation in health and disease}

Rianne D.W. Vaes, Linda van den Berk, Bas Boonen, David P.J. van Dijk, Steven W.M. Olde Damink, Sander S. Rensen Am J Physiol Cell Physiol 315: C598-C607, 2018 


\section{Abstract}

Adaptation of the smooth muscle cell (SMC) phenotype is essential for homeostasis, and is often involved in pathologies of visceral organs (e.g. uterus, bladder, gastrointestinal tract). In vitro studies of the behavior of visceral SMCs under (patho)-physiological conditions are hampered by spontaneous, uncontrolled phenotypic modulation of visceral SMCs under regular tissue culture conditions. We aimed to develop a new visceral SMC culture model that allows controlled phenotypic modulation.

Human uterine SMCs (ULTR and hTERT-HM) were grown to confluency and kept for up to 6 days on regular tissue culture surfaces or basement membrane (BM) matrix coated surfaces, in the presence of $0-10 \%$ serum. mRNA and protein expression and localization of SMC-specific phenotype markers and their transcriptional regulators were investigated by qPCR, Western blot, and immunofluorescence.

Maintaining visceral SMCs confluent for 6 days increased $\alpha$-smooth muscle actin (1.9-fold) and SM22 $\alpha$ (3.1-fold), whereas smooth muscle myosin heavy chain was only slightly upregulated (1.3-fold). Culturing on a BM matrix coated surface further increased these proteins, and also markedly promoted mRNA expression of $\gamma$-smooth muscle actin (15.0fold), smoothelin (3.5-fold), h-caldesmon (5.2-fold), serum response factor (7.6-fold), and myocardin (8.1-fold). Whereas additional serum deprivation only minimally affected contractile markers, platelet-derived growth factor-BB and transforming growth factor beta-1 consistently reduced vs. increased their expression.

In conclusion, we present a simple and reproducible visceral SMC culture system that allows controlled phenotypic modulation towards both the synthetic and the contractile phenotype. This may greatly facilitate the identification of factors that drive visceral SMC phenotypic changes in health and disease. 


\section{Introduction}

The walls of many visceral organs are surrounded by well-developed smooth muscle layers which enable endured constriction or dilation, resulting in transport or storage of fluids. The smooth muscle cells (SMCs) within these layers possess the unique ability to change their phenotype from a so-called contractile state towards a synthetic state. Whereas contractile SMCs are characterized by an elongated spindle-shaped morphology and high expression of specific contractile marker proteins (1), synthetic SMCs show decreased expression of these contractile markers and an increased proliferation rate, high migration rate, and pronounced synthesis of extracellular matrix (ECM) proteins (1).

SMC phenotypic plasticity is essential for adaptation of visceral organs to changes in local environmental conditions in health. For example, during pregnancy, uterine SMC hypertrophy and collagen synthesis is induced by ovarian hormones to cope with the stretch induced by the growing fetus and to maintain a quiescent contractile state until delivery (24). However, SMC phenotypic plasticity can also contribute to disease. An example is inflammatory bowel disease, where increased TNF- $\alpha$ and IL-1 $\beta$ levels reduce intestinal SMC contractility, contributing to intestinal motility problems (5-7). SMC hypertrophy and reduced smooth muscle contractility also underlie the significant increase in bladder mass during partial bladder outlet obstruction secondary to prostatic hyperplasia (8-10).

Given the importance of visceral SMC phenotypic plasticity for the pathogenesis of various disorders as well as normal physiological phenomena, it is pivotal to gain more mechanistic insight into the molecular processes involved and the relevant environmental cues. To this end, a visceral SMC culture system in which the SMC phenotype can be modulated in a controlled way is a prerequisite. Unfortunately, SMCs modulate uncontrollably towards a synthetic phenotype under standard cell culture conditions which lack the physiological micro-environment that normally keeps the cells in a contractile phenotype (11). In particular, a highly structured basal membrane (BM) matrix has been shown to be crucial for maintaining the contractile SMC phenotype (12). Additional elements of the cellular environment, including cell-cell interactions (13), humoral factors (e.g. growth factors, cytokines) (13), and mechanical forces further regulate the SMC phenotype.

We here present the first visceral SMC culture system in which SMCs can be kept in either a highly contractile state or modulated towards the synthetic phenotype in a controlled way, developed by careful optimization of the growth surface, serum concentration, and cell-cell interaction. For the optimization of this culture model, we used well-characterized immortalized human uterine SMC lines which retain in vivo SMC characteristics, such as the expression of contractile and myometrial SMC markers $(14,15)$. This novel culture model can be used to further elucidate the molecular mechanisms driving SMC phenotypic switching and greatly facilitates the study of visceral SMC phenotypic modulation in health and disease. 


\section{Materials and Methods}

\section{Cell culture conditions}

Human uterine SMCs (ULTR cells) (15) were maintained in Dulbecco's Modified Eagle's Medium (DMEM) (Gibco, Cat. No. 42430) supplemented with $4 \mathrm{mM} \mathrm{L-Glutamine,} 10 \%$ (v/v) fetal bovine serum (FBS) (Greiner Bio-one, Cat. No. 758093), and antibiotics (100 units/mL penicillin and $100 \mu \mathrm{g} / \mathrm{ml}$ streptomycin, Gibco). Human myometrial SMCs immortalized with human telomerase reverse transcriptase (hTERT-HM) were a gift from Dr. Jennifer Condon (14). These cells were maintained in DMEM/F12 (Gibco, Cat. No. 31331) supplemented with 10\% FBS, 10 mM HEPES (Gibco, Cat. No. 15630), and antibiotics (100 units/mL penicillin and $100 \mu \mathrm{g} / \mathrm{ml}$ streptomycin). All experiments were performed within 8 passages and both cell lines were maintained at $37^{\circ} \mathrm{C}, 5 \% \mathrm{CO}_{2}$ in a humidified incubator.

For experiments, SMCs were plated at a density of $1 \times 10^{4}$ cells $/ \mathrm{cm}^{2}$ on either standard tissue culture (TC) treated (Eppendorf) or Basement Membrane Matrix (BM) coated (Geltrex Reduced Growth Factor Basement Membrane Matrix, Gibco, Cat No. A14132) surfaces. To obtain BM-coated plates, tissue culture treated multiwell polystyrene plates (Eppendorf) were coated with $100 \mu \mathrm{l} / \mathrm{cm}^{2}$ ice-cold Geltrex (1:50 in DMEM without supplements), and incubated for $2 \mathrm{~h}$ at $37^{\circ} \mathrm{C}$ before plating cells. Subsequently, when cells reached $>90 \%$ confluency (after 48 hours), regular culture medium used for maintaining the cells was replaced by advanced DMEM/F-12 (Gibco, Cat. No. 12634) that allows culturing cells with reduced serum supplementation due to the addition of specific components, including ethanolamine, glutathione, ascorbic acid, insulin, transferrin, and AlbuMAX ${ }^{\circledR}$ lipidrich bovine serum albumin. Advanced DMEM/F-12 medium was supplemented with different serum concentrations (10\%, $2 \%$, or $0 \%$ FBS), $1 \%$ (v/v) GlutaMAX (Gibco, Cat. No. 35050 ), and antibiotics (50 units $/ \mathrm{mL}$ penicillin and $50 \mu \mathrm{g} / \mathrm{ml}$ streptomycin, Gibco). In indicated experiments, SMCs were treated with either PDGF-BB (30 ng/ml, PeproTech, Cat. No. $100-14 B$ ) or TGF- $\beta 1$ (5 ng/ml, PeproTech, Cat. No. 100-21) to induce phenotypic modulation. Medium was refreshed every three days.

\section{Quantitative real-time PCR}

Total RNA was isolated using TRI Reagent (Sigma, St. Louis, MO) according to the manufacturer's protocol. RNA yield was measured with a DeNovix DS-11 spectrophotometer. A total of 750 ng RNA was reversed transcribed to cDNA using the SensiFast cDNA Synthesis Kit according to the manufacturer's instructions (Bioline $\mathrm{GmbH}$, Germany).

To quantify mRNA expression levels of SMC phenotype markers, quantitative realtime PCR (qRT-PCR) analysis was performed on the LightCycler480 (Roche) using a threestep PCR program followed by melting curve analysis. cDNA was amplified with the SensiMix SYBR Hi-Rox kit (Bioline GmbH, Cat. No. QT605-05). Specific primer pairs for each gene were ordered from Sigma and are listed in table 1. Relative gene expression levels were derived from the LinRegPCR (version 2016.1) method (16) and normalized to the geometric average of two reference genes, cyclophylin A (CYPA) and beta-2-microglobulin (B2M). 
Table 1: Quantitative RT-PCR primers

\begin{tabular}{lll}
\hline Gene & $\begin{array}{l}\text { Forward primer } \\
\left(5^{\prime} \rightarrow 3^{\prime}\right)\end{array}$ & $\begin{array}{l}\text { Reverse primer } \\
\left(5^{\prime} \rightarrow 3^{\prime}\right)\end{array}$ \\
\hline CYPA & CTCGAATAAGTTGACTTGTGTTT & CTAGGCATGGGAGGGAACA \\
B2M & TCCATCCGACATTGAAGTTG & CGGCAGGCATACTCATCTT \\
ACTA2 & CCGGGACTAAGACGGGAATC & TTGTCACACACCAAGGCAGT \\
ACTG2 & CGCCCTCGCCACCAG & CCTTGGGATTTAGGGGAGCC \\
TAGLN & AATTGATGGAAACCACCGGG & GGGGAAAGCTCCTTGGAAGT \\
SMTN & ACCATCGAGATCAAGGACGG & CCAGTGTCAGTTCTGCCCTC \\
CALD1 & GCTGTCCTAAAGAAACAGGGAG & GGCTTGTCTTCTTGGAGCTT \\
MYH11 & CATCTACGCCATCGCAGACA & CAGACTCGCCTGTGCATAGAA \\
MYOCD & GGGTCTGAGCATTCCTTGCT & CTGGACGTTTCAGTGGTGGT \\
SRF & TTGCTGAGTGAAGGGGCCTA & AGTTTTCGGGTGGCAAAGGT \\
\hline
\end{tabular}

ACTA2, $\alpha$-smooth muscle actin; ACTG2, $\gamma$-smooth muscle actin; $B 2 M, \beta-2$ microglobulin; $C A L D 1$, caldesmon 1 (primers specific for heavy caldesmon transcript); CYPA, cyclophilin A; MYH11, myosin heavy chain 11; MYOCD, myocardin; SMTN, smoothelin; SRF, serum response factor; TAGLN, smooth muscle protein 22-alpha

\section{Western blotting}

Cells were harvested with lysis buffer containing $10 \mathrm{mM}$ Tris, $100 \mathrm{mM} \mathrm{NaCl}, 1 \mathrm{mM}$ EDTA, $1 \mathrm{mM}$ EGTA, $1 \%$ Triton X-100, $10 \%$ glycerol, $0.1 \%(\mathrm{w} / \mathrm{v})$ SDS, and $0.5 \%(\mathrm{v} / \mathrm{v})$ sodium deoxycholate supplemented with protease inhibitor cocktail tablets (Roche, Mannheim, Germany). Whole cell lysates were incubated on ice for $30 \mathrm{~min}$ and subsequently centrifuged at $16,000 \times \mathrm{g}$ for $30 \mathrm{~min}$ at $4^{\circ} \mathrm{C}$. Supernatant was transferred to a new tube and total soluble protein concentrations were determined using the Pierce BCA Protein Assay Kit (Thermo Scientific ${ }^{\mathrm{TM}}$ ). 4x Laemmli sample buffer ( $250 \mathrm{mM}$ Tris- $\mathrm{HCl}, \mathrm{pH} 6.8,8 \%$ (w/v) SDS, $40 \%$ (v/v) glycerol, $355 \mathrm{mM}$ 2-Mercaptoethanol, and $0.02 \%(\mathrm{w} / \mathrm{v})$ bromophenol blue) was added to the lysate, followed by heating the samples for $5 \mathrm{~min}$ at $95^{\circ} \mathrm{C}$. Equal amounts of protein $(10 \mu \mathrm{g})$ were loaded per lane on a $4-15 \%$ Criterion $^{\mathrm{TM}}$ TGX Stain-Free ${ }^{\mathrm{TM}}$ precast Gel (BioRad). Following electrophoretic separation, the proteins were transferred onto a Transblot Turbo polyvinylidene difluoride (PVDF) transfer membrane (BioRad) by electroblotting on the Trans-Blot ${ }^{\circledR}$ Turbo $^{\mathrm{TM}}$ Blotting System (Bio-Rad). After blocking with $5 \%(\mathrm{w} / \mathrm{v}$ ) BSA (Lampire Biological Laboratories, Cat. No. 7500804), membranes were incubated overnight at $4^{\circ} \mathrm{C}$ with specific monoclonal primary antibodies directed against anti-smooth muscle myosin heavy chain (SM-MHC) (1:1000, Clone BT-562, Alfa Aesar, Cat. No. J64817AMJ), anti- $\alpha$-smooth muscle actin ( $\alpha$-SMA) (1:1000, Clone 1A4, Dako, Cat. No. M0851), and anti-smooth muscle protein 22-alpha (SM22 $\alpha)(1: 1000)$ (the mouse antiSM22 $\alpha$ hybridoma cell line, clone 3E11, was a kind gift from Dr. A. Chiavegato, University of Padua, Italy). After washing with TBS-Tween20 (0.01\%), the blots were probed for 1 hour with an appropriate peroxidase-conjugated secondary antibody (Vector Laboratories, CA, USA) and signals were visualized using SuperSignal West Pico chemiluminescent substrate 
(Thermo Scientific, Rockford, IL) according to the manufacturer's instructions. Images were obtained with a molecular imager (Amersham Imager 600, GE Healthcare Life Sciences) and total band intensity was quantified with ImageQuant TL software (v8.1.0.0, GE Healthcare Life Sciences).

\section{Immunofluorescence}

For double immunofluorescence staining, uterine SMCs were cultured on 18-mm glass coverslips (Thermo Scientific). Cells were fixed for $5 \mathrm{~min}$ in $50 \%(\mathrm{v} / \mathrm{v})$ methanol / $50 \%(\mathrm{v} / \mathrm{v})$ acetone at $-20^{\circ} \mathrm{C}$. Subsequently, cells were rinsed in $1 x$ DPBS (Gibco, Life Technologies), followed by 60 minutes incubation with mouse monoclonal IgG2a antibody recognizing anti$\alpha$-SMA (1:200, Clone 1A4, Dako) and mouse monoclonal IgG1 antibody recognizing SM22 $\alpha$ (1:150, Clone 3E11). Alexa Fluor ${ }^{\circledR} 488$-conjugated goat anti-mouse IgG1 (Invitrogen, Cat. No. A-21121) and Alexa Fluor ${ }^{\circledR}$ 594-conjugated goat anti-mouse IgG2a (Invitrogen, Cat. No. A21135) were used as secondary antibodies. Nuclei were counterstained by 4',6-diamino-2phenylindole (DAPI). Coverslips were mounted in fluorescent mounting medium (DAKO). Digital images were taken using a Leica TCS SP8 $X$ automated inverted confocal microscope equipped with a HC PL APO CS2 63x/1.40 oil objective and processed using ImageJ (version 1.51h).

\section{Statistical analysis}

Data are obtained from two or three independent experiments and results are expressed as mean \pm SEM. Raw data was entered in IBM SPSS 24 for Microsoft Windows ${ }^{\circledR}$ and statistical analyses were performed using the non-parametric Mann-Whitney $U$ test to compare differences between two groups. In case of more than two groups, the non-parametric Kruskal-Wallis test was applied followed by Dunn's post-hoc testing with Bonferroni correction. A p-value of $p<0.05$ was considered statistically significant. 


\section{Results}

\section{Effect of prolonged cell-cell contact on SMC-specific contractile markers}

Reduced cell-cell contact as a consequence of culturing SMCs at low density has previously been shown to result in decreased expression of contractile marker proteins (13). At confluence, vascular SMCs lose their proliferative capacity and concurrently increase their expression of a panel of SMC specific contractile proteins like $\alpha$-SMA, SM22 $\alpha$, and SM-MHC. To investigate whether visceral SMCs exhibit a similar cellular behavior, we cultured ULTR cells towards a confluent state and maintained them for 3 or 6 days in the presence of $10 \%$ FBS, before analyzing contractile marker expression. These immortalized cells have previously been shown to acquire characteristics of the synthetic SMC phenotype when cultured towards a subconfluent state, including a high proliferation rate, decreased cell size, and irregular distribution of $\alpha$-actin filaments (15). Culturing ULTR SMCs for 3 days in a
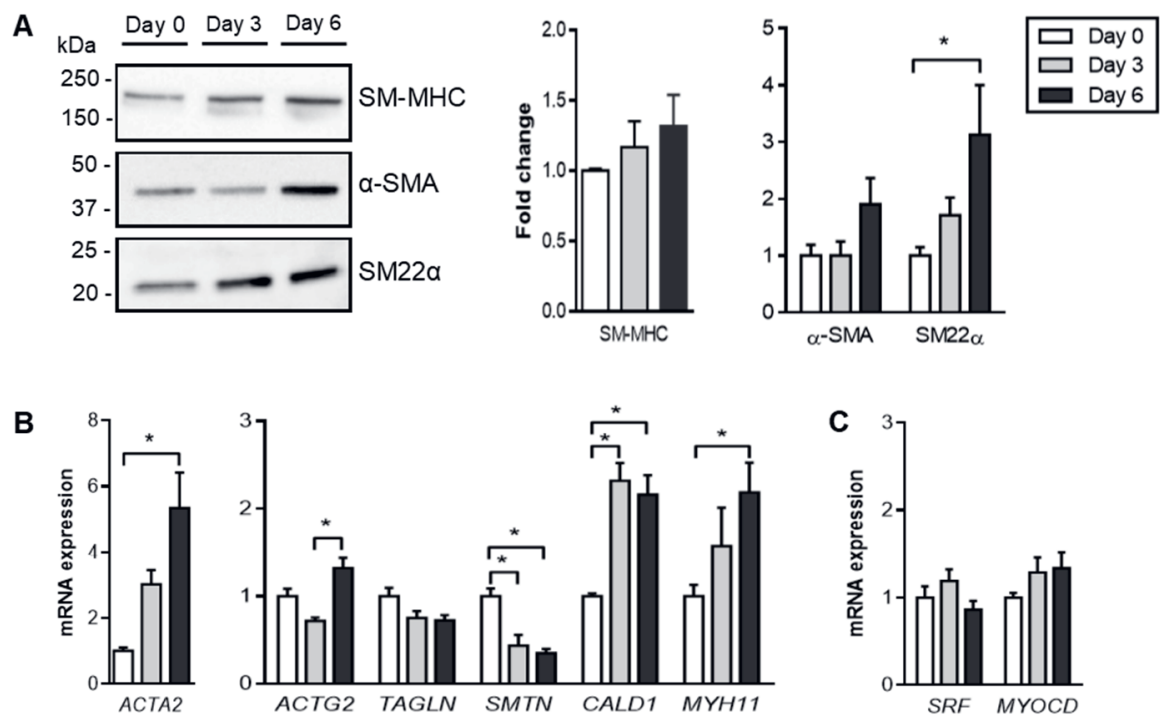

Figure 1: Expression of SMC-specific contractile markers in confluent visceral SMCs. Visceral SMCS (ULTR cells) were cultured on a TC-coated surface in medium containing $10 \%$ FBS. (A) Protein expression of SM-MHC, $\alpha$-SMA, and SM22 $\alpha$ were determined by Western Blot analysis. All samples from each independent experiment (SM-MHC, $\mathrm{n}=6 ; \alpha-\mathrm{SMA}, \mathrm{SM} 22 \alpha, \mathrm{n}=9$ ) were derived the same time and processed in parallel. mRNA expression levels of (B) contractile (ACTA2, ACTG2, TAGLN, $S M T N, C A L D 1$, and MYH11) and (C) transcriptional regulatory (SRF, MYOCD) genes were determined and expressed relative to expression levels at day 0 . Data were normalized to CYPA and B2M reference genes. Results from each time-point were obtained from two (CALD1, MYH11; $\mathrm{n}=6$ ) or three (ACTA2, ACTG2, TAGLN, SMTN, SRF, MYOCD; $\mathrm{n}=9$ ) independent experiments and are presented as mean \pm SEM. ( ${ }^{*} p<0.05$ compared to D0, Kruskal-Wallis test followed by Dunn's post-hoc testing with Bonferroni correction). ACTA2, alpha-smooth muscle actin; ACTG2, gamma-smooth muscle actin; TAGLN, smooth muscle protein 22-alpha; SMTN, smoothelin; CALD1, caldesmon; MYH11, smooth muscle myosin heavy chain; SRF, serum response factor; $M Y O C D$, myocardin. 
confluent state resulted in slightly increased SM-MHC and SM $22 \alpha$ protein levels, but these differences were not significant (Fig. 1A). However, contractile protein levels continued to increase and after 6 days, SM22 $\alpha$ protein levels were significantly increased by 3.1-fold $(p=0.01)$, and $\alpha$-SMA protein levels tended to be significantly increased (1.9-fold, $p=0.09$ ). These protein data indicate that increasing cell-cell contact only minimally promotes the contractile phenotype in visceral SMCs. However, mRNA expression of many contractile marker genes was significantly increased after 6 days (Fig. 1B): ACTA2 (5.3-fold, $\mathrm{p}<0.001$ ), ACTG2 (1.3-fold, $\mathrm{p}<0.001$ ), CALD1 (2.2-fold, $\mathrm{p}=0.03$ ), and MYH11 (2.2-fold, $\mathrm{p}=0.04)$. This suggests that increased cell-cell contact initiates transcriptional upregulation of contractile genes, although this was not sufficient to induce differences at the protein level. Nevertheless, transcription of serum response factor (SRF) and myocardin (MYOCD), genes important for transcriptional regulation of the contractile phenotype markers, was not induced by prolonged confluency (Fig. 1C).

Basement membrane components promote the visceral SMC contractile phenotype Since the interaction of cells with the extracellular microenvironment is known to influence vascular SMC differentiation, migration, proliferation, and survival (17), we next investigated whether addition of basal membrane matrix (BM) components could further stimulate the expression of contractile proteins in visceral SMCs. In comparison with cells grown on TC coated surfaces (Fig. 1), SMCs cultured on a BM coated surface showed a higher increase of ACTA2 gene expression after 6 days of culturing at confluency (TC: 2.9fold, $p<0.001$ vs. BM: 8.2 -fold, $p=0.002$ ) (Fig. 2A). More importantly, cells grown on BM markedly upregulated their expression of the more advanced contractile phenotype markers (ACTG2, 15.0-fold, $p=0.002$; TAGLN (3.2-fold, $p=0.7$ ); SMTN (3.5-fold, $p=0.002$; CALD1, 5.2-fold, $\mathrm{p}=0.004$ ) (Fig. 2A) compared to the SMCs grown on TC coated surfaces (Fig. $1 B)$. These inductions were also observed at the protein level, where expression of $\alpha$-SMA was increased by 3.9 -fold ( $p<0.001$ ), and expression of SM $22 \alpha$ by 4.8 -fold $(p<0.001$ ) (Fig. $2 \mathrm{~B})$. In addition, SM-MHC protein levels were significantly enhanced in cells grown on BM matrix (2.2-fold, $p=0.002$ ). Moreover, mRNA expression of transcriptional regulators of the contractile phenotype was highly upregulated in SMCs cultured on BM matrix (SRF: 7.6-fold, $p=0.002$; MYOCD: 8.1-fold, $p=0.002$ ) (Fig. 2C). 

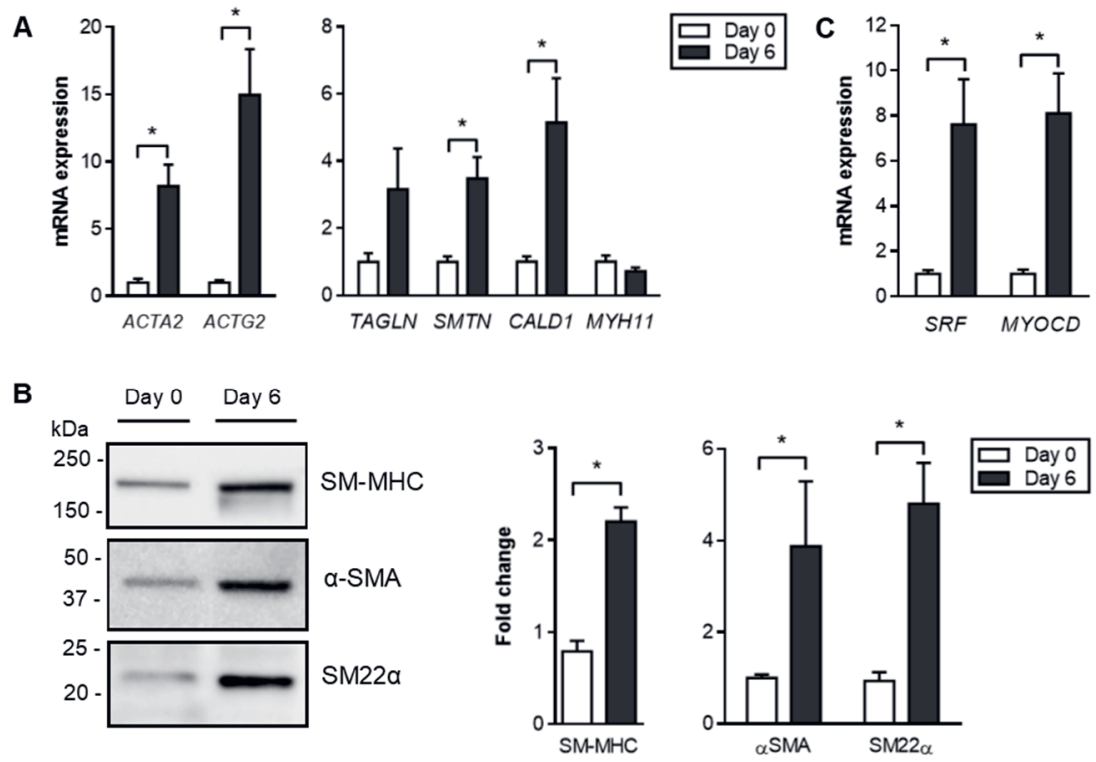

Figure 2: Culturing visceral SMCs on a basement membrane matrix coated surface promotes a contractile phenotype. Visceral SMCS (ULTR) were seeded on a BM-coated growth surface in medium containing 10\% FBS. mRNA expression levels of (A) contractile (ACTA2, ACTG2, TAGLN, SMTN, CALD1, and $M Y H 11)$ and (B) transcriptional regulatory $(S R F, M Y O C D)$ genes were determined and expressed as relative expression levels with day 0 samples set at 1 . Data were normalized to CYPA and B2M reference genes. (C) Protein expression levels of SM-MHC, $\alpha$-SMA, and SM22 $\alpha$ were determined by Western Blot analysis. All samples from each independent experiment were derived the same time and processed in parallel (SM-MHC, $n=6 ; \alpha-S M A, S M 22 \alpha, n=9$ ). Results are presented as mean \pm SEM (Mann-Whitney U test, $* p<0.05$ ).

\section{Effect of serum concentrations on the phenotype of SMCs grown on BM matrix}

Growth factors present in FBS are known to stimulate cell proliferation, migration, and matrix protein synthesis, which are all characteristics of the synthetic SMC phenotype. We therefore investigated whether reducing serum concentrations could further promote the contractile phenotype of visceral SMCs grown on BM matrix for 6 days. In comparison to the $10 \%$ FBS condition, only $\alpha$-SMA protein levels were slightly increased (1.3-fold, Fig. $3 \mathrm{~A}$ ) when cells were cultured in $2 \% \mathrm{FBS}$ or $0 \% \mathrm{FBS}$, although these increases were not significant. However, SM-MHC and SM22 $\alpha$ protein concentrations were similar when cells were grown in $10 \%$ vs. $2 \%$ FBS (Fig. 3A), and complete serum deprivation even caused significantly decreased SM-MHC (0.7-fold, $p=0.03$ ) and SM $22 \alpha$ protein expression (0.4-fold, $p=0.005$ ) (Fig. 3A). Of note, complete serum deprivation seemed to reduce cell viability as indicated by cell morphology and the appearance of floating cells (data not shown). At the mRNA level, reducing serum concentrations did not increase expression of contractile phenotype markers in comparison with the $10 \%$ FBS condition (Fig. 3B), except for CALD1, which was slightly upregulated. This suggests that the known inhibitory effect of the growth factors on the SMC contractile phenotype is blunted when cells are grown on a BM matrix. 
A
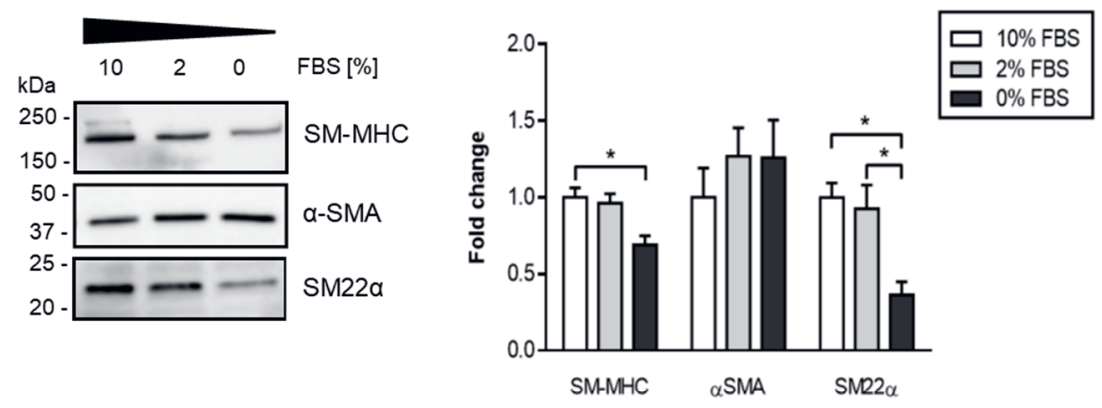

B

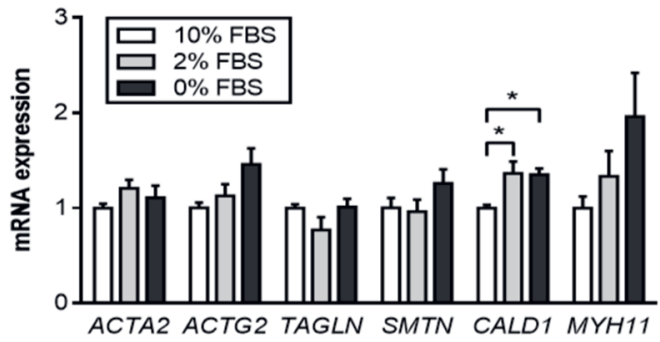

Figure 3: Effect of serum on contractile marker expression in visceral SMCs grown on BM. Visceral SMCs (ULTR cells) were cultured on a BM-coated growth surface. When cells reached $>90 \%$ confluency, growth medium was replaced by differentiation medium containing reduced serum concentrations $(10 \%, 2 \%$, or $0 \%$ FBS) for 6 days. (A) Protein expression levels of SM-MHC, $\alpha$-SMA, and $\mathrm{SM} 22 \alpha$ were determined by Western Blot analysis. All samples from each independent experiment were derived the same time and processed in parallel (SM-MHC, $n=6 ; \alpha-S M A, S M 22 \alpha, n=9$ ). (B) mRNA expression levels of ACTA2, ACTG2, TAGLN SMTN, CALD1, and MYH11 were determined and are expressed as relative expression with day 0 samples set at 1 . Data were normalized to CYPA and $\mathrm{B} 2 \mathrm{M}$ reference genes. Results from each time-point were obtained from two (CALD1, MYH11, $\mathrm{n}=6$ ) or three (ACTA2, ACTG2, TAGLN, SMTN; $\mathrm{n}=9$ ) independent experiments and are presented as mean \pm SEM. (Kruskal-Wallis test followed by Dunn's post-hoc testing with Bonferroni correction, ${ }^{*} p<0.05$ ).

\section{Visceral SMCs undergo morphological and structural changes within an optimized} SMC culture environment

The results presented above indicate that by optimizing culture conditions, visceral SMCs maintain a relatively contractile phenotype characterized by high contractile marker protein expression. Consistent with this, the SMCs cultured at these optimized conditions underwent the typical morphological changes that SMCs undergo during acquisition of a contractile phenotype. Whereas a heterogeneous population of rhomboid- and elongated spindle-shaped cells was present at day 0 , cells cultured on basement membrane matrix proteins acquired the typical contractile phenotype-associated elongated spindle-shaped morphology upon 6 days of culturing (Fig. 4A). These morphological changes were accompanied by pronounced rearrangements of the contractile apparatus: $\alpha-S M A$ filaments in synthetic rhomboid SMCs ran crisscross through the cytoplasm (Fig. 4B, a-c) whereas they reorganized to run parallel to the long axis in elongated spindle-shaped SMCs 
A
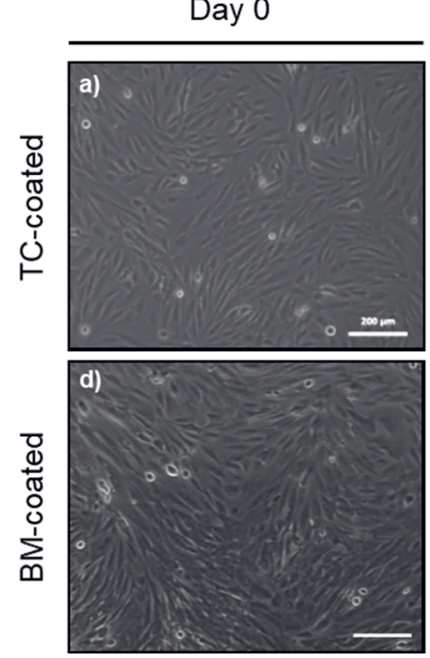

B
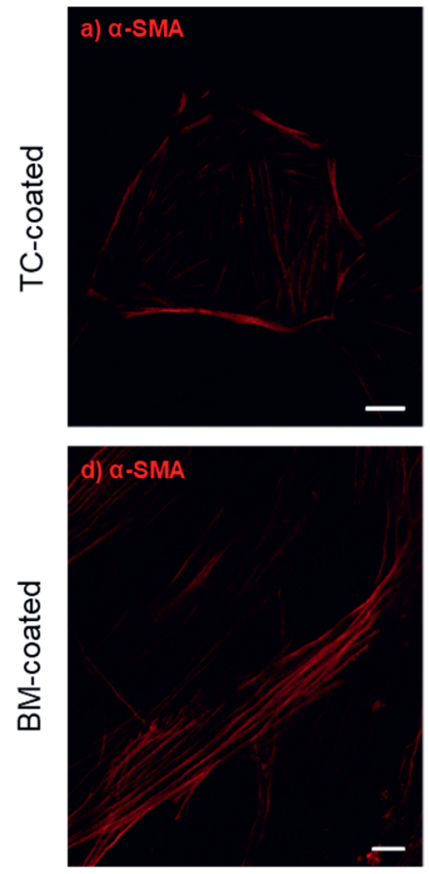

Day 6
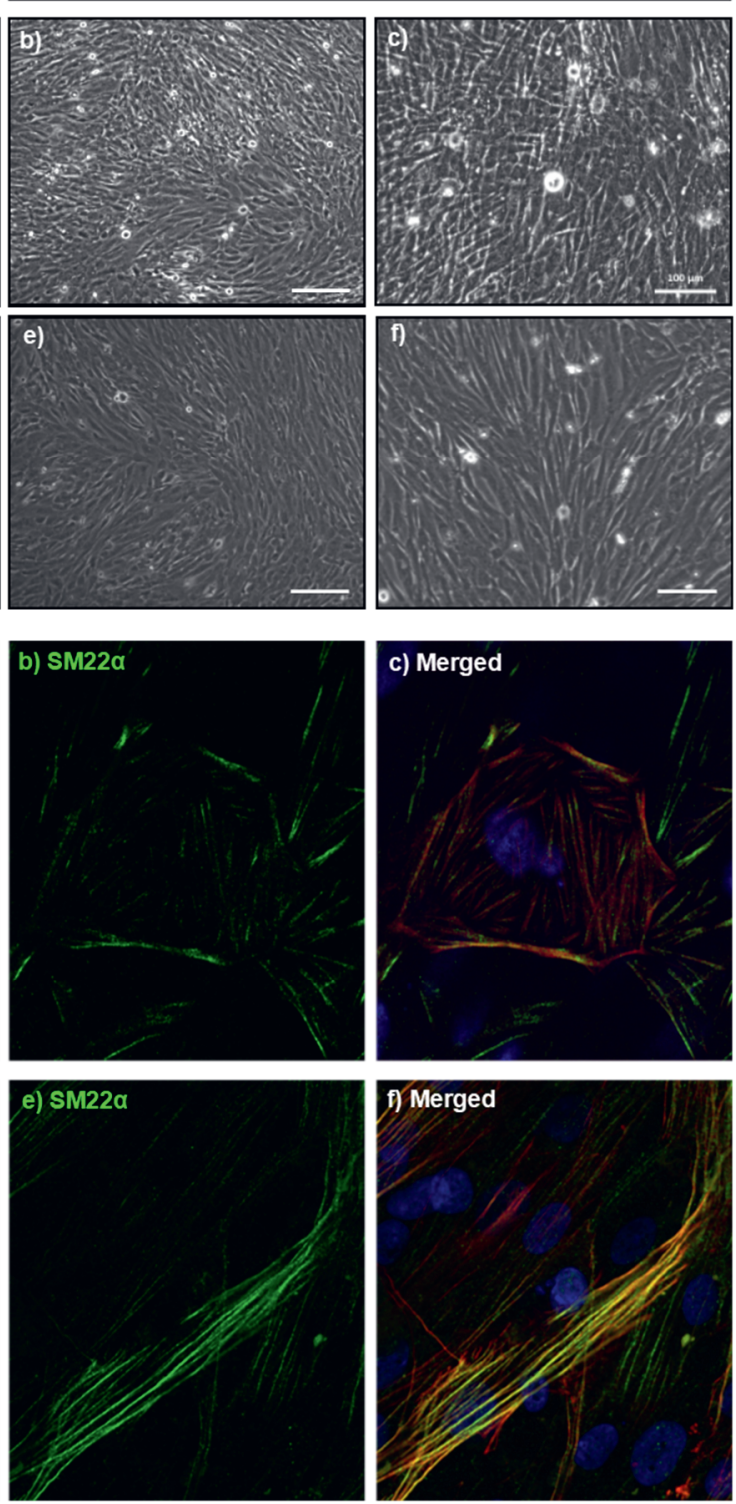

Figure 4: Controlled modulation of morphological and structural characteristics of visceral SMCs. Visceral SMCs (ULTR cells) were grown on either TC-coated or BM-coated surfaces. When cells reached $>90 \%$ confluency, confluent cells were kept for 6 days in culture medium containing either $10 \%$ or $2 \%$ FBS, respectively. (A) Phase-contrast microphotographs ( $a-b, d-e$, bar $=200 \mu \mathrm{m}$ and $c, f$, bar $=100 \mu \mathrm{m})$ and $(\mathbf{B})$ double immunofluorescence staining (bar $=10 \mu \mathrm{m})$ showing $\alpha$-SMA and SM22 $\alpha$ expression in visceral SMCs cultured on TC-coated $(a-c)$ and BM-coated $(d-f)$ surfaces. Nuclei are stained in blue by DAPI. 
(Fig. 4B, d-f). Moreover, SM22 $\alpha$ was abundantly present along the actin filaments in contractile SMCs (Fig. 4B, d-f) compared to synthetic SMCs, in which SM22 $\alpha$ was only marginally expressed and appeared to show less colocalization with $\alpha$-SMA filaments (Fig. $4 B, a-c)$.

The contractile phenotype of visceral SMCs grown on BM can be modulated in a controlled way

To further show the potential of this culture system for modelling disease states characterized by SMC phenotypic changes, we stimulated the cells with factors known to influence the vascular SMC phenotype (PDGF-BB and TGF- $\beta 1$ ). In vascular SMCs, PDGF-BB has been shown to suppress the contractile phenotype while TGF- $\beta 1$ stimulates it $(18,19)$. PDGF-BB and TGF- $\beta 1$ stimulation greatly influenced both protein and gene expression levels of contractile SMC markers of visceral SMCs cultured for 6 days on a BM-coated growth surface with $2 \%$ FBS. SMCs incubated for $48 \mathrm{~h}$ with PDGF-BB showed reduced protein levels of $\alpha$-SMA (0.5-fold, $p=0.048)$, and SM $22 \alpha(0.6$-fold, $p=0.4)$ tended to be decreased as well

A
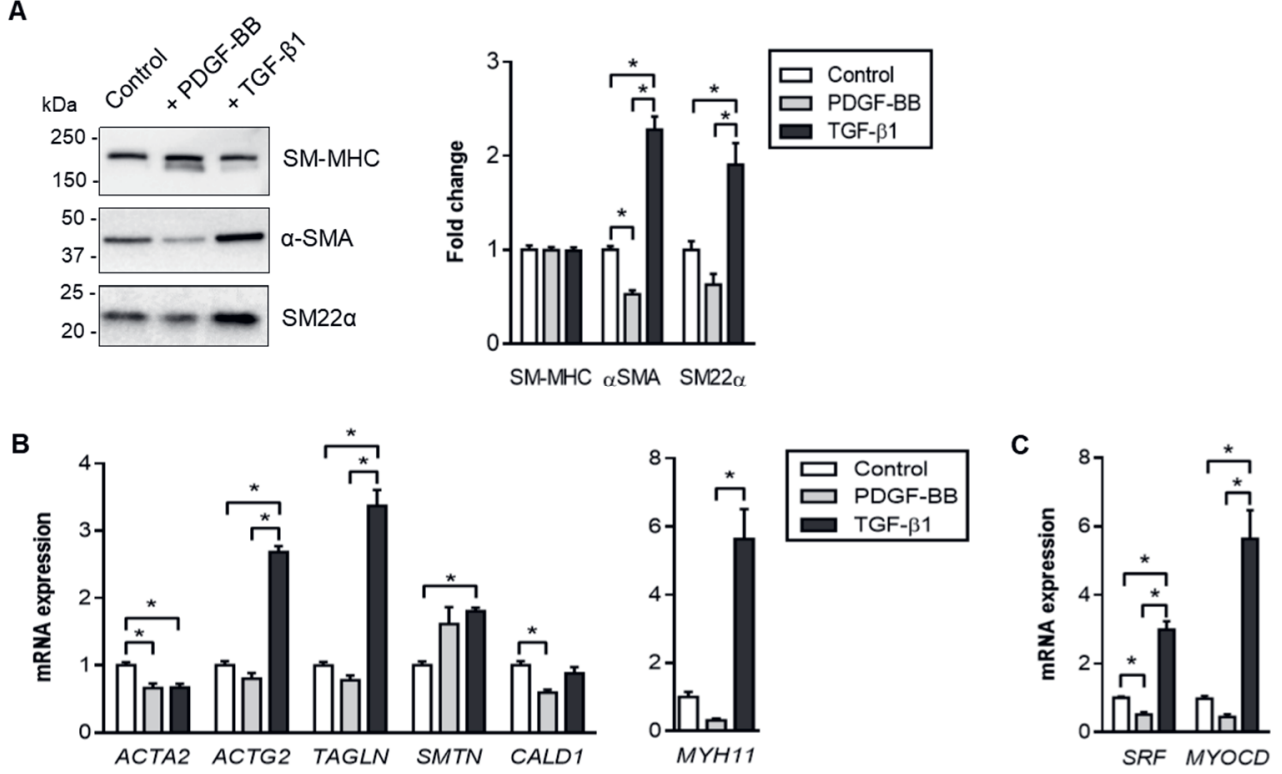

Figure 5: Controlled modulation of the visceral SMC phenotype by PDGF-BB and TGF- $\beta 1$. Visceral SMCs (ULTR cells) were cultured for 6 days with $2 \%$ FBS to induce the expression of contractile SMC markers. After 6 days, cells were incubated with either PDGF-BB (30 ng/ml) or TGF- $\beta 1$ ( $5 \mathrm{ng} / \mathrm{ml})$. (A) Protein expression levels of SM-MHC, $\alpha$-SMA, and SM22 $\alpha$ were determined by Western Blot analysis after 48h. mRNA expression levels of (B) contractile (ACTA2, ACTG2, TAGLN, SMTN, CALD1, and $M Y H 11$ ) and (C) transcriptional regulatory (SRF, MYOCD) genes were determined after 24h. mRNA expression levels are expressed as relative expression levels with untreated samples set at 1 . Data were normalized to CYPA and B2M reference genes. Results are obtained from two (CALD1, MYH11; $\mathrm{n}=6$ ) or three (ACTA2, ACTG2, TAGLN, SMTN, SRF, MYOCD; $\mathrm{n}=9$ ) independent experiments (mean \pm $\mathrm{SEM}$, Kruskal-Wallis test followed by Dunn's post-hoc testing with Bonferroni correction, $* \mathrm{p}<0.05$ ). 
(Fig. 5A). Conversely, stimulation with TGF- $\beta 1$ further increased protein expression of both $\alpha$-SMA (2.3-fold, $p=0.048$ ) and SM22 $\alpha$ (1.9-fold, $p=0.04$, Fig. 5A) in cells that already had a contractile phenotype as a result of culturing on BM matrix. Although protein levels of $\alpha-$ SMA and SM22 $\alpha$ were altered after PDGF-BB and TGF- $\beta 1$ stimulation, no differences in SMMHC protein levels were observed. On the other hand, MYH11 mRNA levels were markedly induced by TGF- $\beta 1$ treatment, along with induction of ACTG2, TAGLN, SMTN, SRF, and MYOCD (Fig. 5B-C). Treatment with PDGF-BB provoked significant reductions in ACTA2, CALD1, and SRF levels, as well as smaller, non-significant reductions of ACTG2, TAGLN, MYH11, and MYOCD. Overall, these data not only indicate that visceral SMCs can be kept in a contractile phenotype by culturing them on BM matrix for a prolonged time, but also show that their phenotype can still be modulated in a controlled way towards both a more contractile and a more synthetic state.

Validation of culture conditions for controlled phenotypic modulation of visceral smooth muscle cells

To demonstrate that the culture conditions defined above can also be applied to study phenotypic modulation in other visceral SMCs, we validated our culture system using an independent uterine smooth muscle cell line (hTERT-HM). These highly proliferative immortalized SMCs have been shown to retain smooth muscle-specific markers (e.g. $\alpha$ SMA, $h$-caldesmon, and calponin) and display an elongated phenotype, potentially indicating a more contractile phenotype compared to ULTR cells (14). We first compared the morphology of confluent hTERT-HM cells grown on either TC or BM-coated surfaces (Fig. 6A). SMCs cultured on TC-coated surfaces adopted the typical rhomboid-shaped morphology after 6 days, indicative of the synthetic phenotype. In accordance with previous results obtained using ULTR cells, we observed a homogeneous population of elongatedspindle shaped cells when hTERT-HM SMCs were cultured on BM-coated surfaces, consistent with modulation towards the contractile phenotype. Next, we validated the changes in protein expression levels of SMC-specific contractile markers upon culturing on TC-coated versus BM-coated surfaces (Fig. 6B). Similar to what was observed for ULTR SMCs, hTERT-HM cells cultured on BM-coated surfaces showed marked upregulation of SMMHC (2.1-fold, $p<0.001$ ), $\alpha$-SMA (4.2-fold, $p<0.001$ ), and SM22 $\alpha$ (2.3-fold, $p<0.001$ ) protein compared to hTERT-HM cells cultured on TC-coated surfaces. Finally, we investigated whether contractile hTERT-HM SMCs grown on BM were responsive to PDGF-BB and TGF$\beta 1$ to stimulate phenotypic modulation. Although these cells only slightly decreased gene and protein expression levels of SMC-specific contractile markers after PDGF-BB treatment, significantly increased levels of these markers were observed after stimulation with TGF- $\beta 1$ (Fig. 6 C-E). Altogether, these results corroborate the data obtained with the ULTR cells, and demonstrate that the culture conditions defined above can be applied to study phenotypic modulation in other visceral SMC lines. 
A

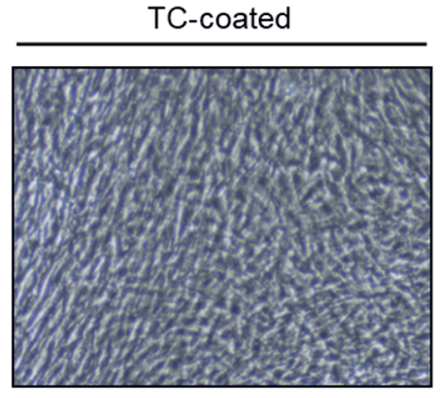

BM-coated

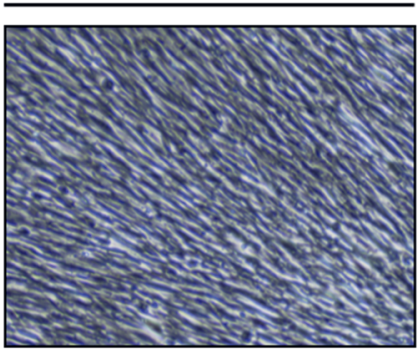

B
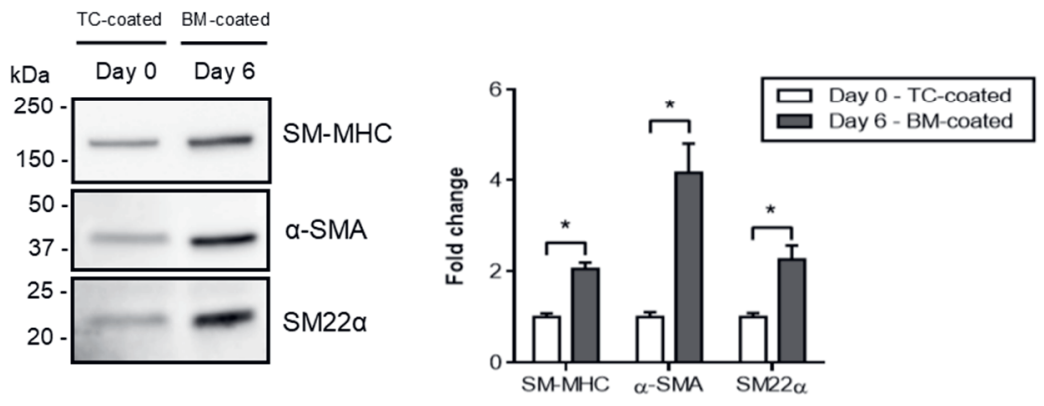

C
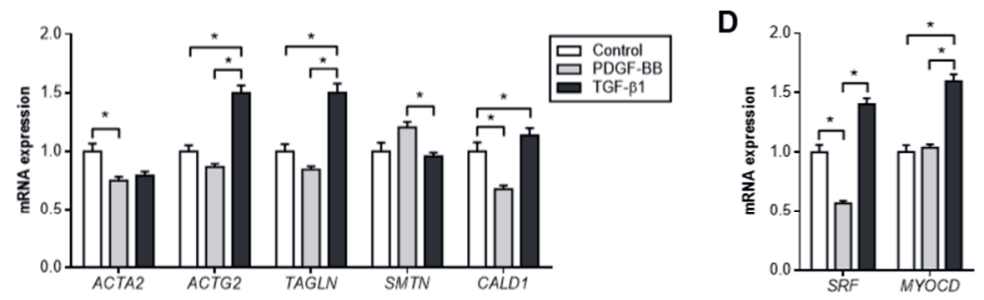

E
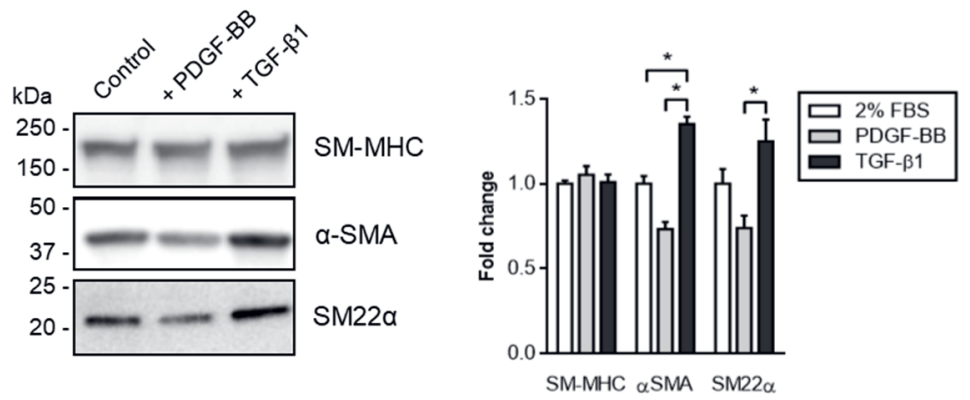

Figure 6: Validation of culture conditions for controlled phenotypic modulation of visceral smooth muscle cells using human hTERT-HM cells. Human hTERT-HM SMCs were grown on either TC-coated or BM-coated surfaces. When cells reached $>90 \%$ confluency (day 0 ), confluent cells were kept for 6 days in culture medium containing either $10 \%$ or $2 \%$ FBS, respectively. (A) Phase-contrast microphotographs. Confluent SMCs grown on TC-coated surfaces (left) and confluent SMCs grown on BM-coated surfaces (right) at day 6. (B) Protein expression levels of SM-MHC, $\alpha$-SMA, and SM22 $\alpha$ expressed by hTERT-HM. Human hTERT-HM SMCs were grown on either TC-coated or BM-coated surfaces. When cells reached $>90 \%$ confluency (day 0 ), confluent cells were kept for 6 days in 
$<$ Continue Figure 6. culture medium containing either $10 \%$ or $2 \%$ FBS, respectively. Protein expression levels were determined by Western Blot analysis. All samples from each independent experiment ( $n=3$, each with biological triplicates) were derived at the same time and processed in parallel. Results are presented as mean \pm SEM (Mann-Whitney $U$ test, ${ }^{*} p<0.05$ ). mRNA expression levels of (C) contractile (ACTA2, ACTG2, TAGLN, SMTN, and CALD1) and (D) transcriptional regulatory (SRF, MYOCD) markers were determined in hTERT-HM SMCs treated with PDGF-BB and TGF- $\beta 1$ (24h). Results are expressed as relative expression with untreated samples set at 1 . Data were normalized to CYPA and B2M reference genes and are presented as mean \pm SEM. (E) Protein expression levels of SM-MHC, $\alpha$-SMA, and SM22 $\alpha$ were determined by Western Blot analysis after hTERT-HM were treated for $48 \mathrm{~h}$ with either PDGF-BB or TGF- $\beta 1$. (Kruskal-Wallis test followed by Dunn's post-hoc testing with Bonferroni correction, $* \mathrm{p}<0.05)$ 


\section{Discussion}

The present study describes culture conditions that can be applied to keep visceral SMCs in a highly contractile state, while still allowing phenotypic modulation towards either a more contractile or synthetic phenotype in a controlled way. This is the first study in which the phenotypic response of visceral SMCs to combinations of micro-environmental factors, including cell-cell interactions, cell-BM interactions, and serum concentrations was systematically investigated. Whereas serum deprivation did not significantly affect the SMC phenotype, increasing cell-cell interactions and cell-BM interactions greatly promoted the acquisition and maintenance of a contractile phenotype.

The vast majority of previous studies on visceral SMC phenotypic modulation used primary cells, which have important drawbacks since they have a limited life span and display uncontrolled phenotypic modulation towards the synthetic phenotype during the initial stages of culturing $(11,20)$. Together with differences in the (epi)genetic background of the donor, this greatly hampers reproducibility of studies. Our use of established immortalized SMC lines ensures a consistent supply of cells with similar properties while also reducing practical logistic issues associated with isolating cells from tissue.

Vascular and visceral SMCs have been shown to display a substantially different response to changes in microenvironmental stimuli $(21),(22,23)$. In addition, they express different actin isoforms (24). Whereas $\alpha$-SMA is the classical marker of early vascular SMC differentiation (25), $\gamma$-SMA is the dominant actin isoform in visceral SMCs (esophagus, stomach, intestine, bladder, and uterus) (26). Although we observed increased $\alpha$-SMA expression in confluent visceral SMCs grown on a regular tissue culture-treated growth surface, $\gamma$-SMA responded particularly strong to the addition of basement membrane matrix and TGF- $\beta 1$. This may indicate that the optimized culture conditions described here more closely resemble the in vivo visceral SMC microenvironment which is associated with relatively higher $\gamma$-SMA expression. $\gamma$-SMA and other SMC contractile genes like SM $22 \alpha$, smoothelin, and caldesmon are transcriptionally regulated by SRF and its muscle-restricted cofactor myocardin $(27,28)$. Disruption of the SRF-myocardin complex in vascular SMCS results in an overall decrease of contractile marker proteins (29). In line with this, loss of myocardin causes profound defects in the vasculature. Since the gastrointestinal and genitourinary tracts are also affected in myocardin knockouts, myocardin is thought to be required in both vascular and visceral SMCs (30). Our results confirm expression of SRF and myocardin in uterine SMCs in vitro and show that their levels increase in parallel with contractile SMC markers when confluent visceral SMCs are cultured on BM matrix. Importantly, we observed only low expression of SRF and myocardin in SMCs cultured on tissue culture-treated plastic.

The basement membrane that surrounds SMCs in vivo consists of laminin, collagen IV, heparin sulfate proteoglycan, entactin, and various growth factors (i.e. fibroblast growth 
factor, TGF- $\beta 1$, epidermal growth factor, etc.). When SMCs are isolated from tissues, this extracellular environment is lost, and contractile and cytoskeletal filaments are reorganized in order to adapt to a 2D culture environment, resulting in the acquisition of a synthetic phenotype (31). However, re-introduction of basement membrane components like laminin and collagen IV has been shown to promote the contractile phenotype in vascular SMCS (31-34). To mimic the in vivo microenvironment associated with the contractile phenotype in culture, we used a growth factor-reduced basement membrane matrix (Geltrex ${ }^{\mathrm{TM}}$ ) that is purified from the Engelbreth-Holm-Swarm tumor. The composition of this matrix is similar to the basal lamina that surrounds SMCs in vivo (35). Since we observed marked induction of all contractile markers and their transcriptional regulators upon culturing human uterine SMCs on a BM coated growth surface, components of the basement membrane matrix appear to induce a contractile phenotype in visceral SMCs as well. Furthermore, the stiffness of the basement membrane coated surface is likely to be different from that of regular uncoated surfaces used to culture cells, which also affects the SMC phenotype. For example, SMCs are known to proliferate more quickly on stiff compared to soft substrates, indicating a synthetic phenotype (36). In line with this, we found that the relatively soft surface provided by the basement membrane coating was associated with a more contractile phenotype.

Withdrawal of FBS from the culture medium is a commonly used method to initiate differentiation of many different cell types, including vascular SMCs $(13,37,38)$. For example, Solodushko and colleagues showed that confluent serum starved vascular SMCs respond to increased serum concentrations with increased cell cycle progression and increased DNA synthesis, indicating that serum factors overrule contact inhibition in confluent vascular SMCs (39). Similar results were reported by Kato and colleagues, who showed that high FBS concentrations promoted cell cycle progression and increased total protein and collagen synthesis rates in comparison to low serum conditions in postconfluent rat aortic SMCs (13). Along this line, we expected that serum deprivation would promote a shift towards the contractile phenotype. However, reducing serum concentrations had only minimal effects on the expression of contractile markers in visceral SMCs cultured on basement membrane matrix. This may be explained by the fact that basement membrane matrix proteins inhibit growth factor-induced proliferation and therefore inhibit the shift towards the synthetic phenotype $(40,41)$.

Previous studies have shown that cell-cell contact induces growth arrest of vascular SMCs which subsequently results in the induction of smooth muscle specific contractile proteins $(13,42,43)$. Even in the presence of $10 \%$ FBS, density-induced growth arrest has been shown to be associated with elevated $\alpha$-SMA expression in vascular SMCs $(13,43)$. In contrast to this, we only observed minimally increased expression of contractile protein markers and their transcriptional regulators in visceral SMCs kept confluent for 6 days. This indicates that visceral SMCs are less responsive to the increases in cell-cell interactions in the post-confluent state compared to vascular SMCs. 
A limitation of our culture model is the absence of non-muscle cells that regulate the contractile functionality of SMCs, such as neuronal cells and the interstitial cells of Cajal. Furthermore, it should be noted that we cannot distinguish between cell hypertrophy and increased cell proliferation as a cause of smooth muscle marker protein differences between the different culture conditions. In addition, immortalized cell lines can acquire different characteristics compared to normal cells due to activation of different gene expression programs. Nevertheless, SMC plasticity remained intact in our model since contractile marker expression could be both induced and repressed.

In conclusion, we developed a novel visceral SMC culture system in which confluent visceral SMCs can be kept in a contractile phenotype by culturing on a BM coating under low serum conditions. This contractile phenotype can be further modulated towards a more contractile or a more synthetic state by TGF- $\beta 1$ or PDGF-BB stimulation. As such, this SMC culture system represents a highly suitable model to study phenotypic modulation of visceral SMCs in health and disease. 


\section{References}

1. Rensen SS, Doevendans PA, van Eys GJ. Regulation and characteristics of vascular smooth muscle cell phenotypic diversity. Neth Heart J. 2007;15(3):100-8.

2. Bdolah Y, Segal A, Tanksale P, Karumanchi SA, Lecker SH. Atrophy-related ubiquitin ligases atrogin-1 and MuRF-1 are associated with uterine smooth muscle involution in the postpartum period. Am J Physiol Regul Integr Comp Physiol. 2007;292(2):R971-6.

3. Riemer RK, Heymann MA. Regulation of uterine smooth muscle function during gestation. Pediatr Res. 1998;44(5):615-27.

4. Henell F, Ericsson JL, Glaumann H. An electron microscopic study of the post-partum involution of the rat uterus. With a note on apparent crinophagy of collagen. Virchows Arch B Cell Pathol Incl Mol Pathol. 1983;42(3):271-87.

5. Ohama T, Hori M, Fujisawa M, Kiyosue M, Hashimoto $M$, Ikenoue $Y$, et al. Downregulation of $\mathrm{CPI}-17$ contributes to dysfunctional motility in chronic intestinal inflammation model mice and ulcerative colitis patients. J Gastroenterol. 2008;43(11):858-65.

6. Ohama T, Hori M, Momotani E, Iwakura $\mathrm{Y}$, Guo F, Kishi H, et al. Intestinal inflammation downregulates smooth muscle CPI-17 through induction of TNF-alpha and causes motility disorders. Am J Physiol Gastrointest Liver Physiol. 2007;292(5):G1429-38.

7. Ohama T, Hori M, Sato K, Ozaki H, Karaki H. Chronic treatment with interleukin-1beta attenuates contractions by decreasing the activities of CPI-17 and MYPT-1 in intestinal smooth muscle. J Biol Chem. 2003;278(49):48794-804.

8. Zhang EY, Stein R, Chang S, Zheng Y, Zderic SA, Wein AJ, et al. Smooth muscle hypertrophy following partial bladder outlet obstruction is associated with overexpression of non-muscle caldesmon. Am J Pathol. 2004;164(2):601-12.

9. Burkhard FC, Lemack GE, Zimmern PE, Lin VK, McConnell JD. Contractile protein expression in bladder smooth muscle is a marker of phenotypic modulation after outlet obstruction in the rabbit model. J Urol. 2001;165(3):963-7.

10. Bing W, Chang S, Hypolite JA, DiSanto ME, Zderic SA, Rolf L, et al. Obstruction-induced changes in urinary bladder smooth muscle contractility: a role for Rho kinase. Am J Physiol Renal Physiol. 2003;285(5):F990-7.

11. Chamley-Campbell J, Campbell GR, Ross R. The smooth muscle cell in culture. Physiol Rev. 1979;59(1):1-61.

12. Stegemann JP, Hong H, Nerem RM. Mechanical, biochemical, and extracellular matrix effects on vascular smooth muscle cell phenotype. J Appl Physiol (1985). 2005;98(6):2321-7.

13. Kato S, Shanley JR, Fox JC. Serum stimulation, cell-cell interactions, and extracellular matrix independently influence smooth muscle cell phenotype in vitro. Am J Pathol. 1996;149(2):687-97.

14. Condon J, Yin S, Mayhew B, Word RA, Wright WE, Shay JW, et al. Telomerase immortalization of human myometrial cells. Biol Reprod. 2002;67(2):506-14.

15. Perez-Reyes N, Halbert CL, Smith PP, Benditt EP, McDougall JK. Immortalization of primary human smooth muscle cells. Proc Natl Acad Sci U S A. 1992;89(4):1224-8.

16. Ruijter JM, Ramakers C, Hoogaars WM, Karlen Y, Bakker O, van den Hoff MJ, et al. Amplification efficiency: linking baseline and bias in the analysis of quantitative PCR data. Nucleic Acids Res. 2009;37(6):e45.

17. Raines EW. The extracellular matrix can regulate vascular cell migration, proliferation, and survival: relationships to vascular disease. Int J Exp Pathol. 2000;81(3):173-82.

18. Hautmann MB, Madsen CS, Owens GK. A transforming growth factor beta (TGFbeta) control element drives TGFbeta-induced stimulation of smooth muscle alpha-actin gene expression in concert with two CArG elements. J Biol Chem. 1997;272(16):10948-56. 
19. Dandre F, Owens GK. Platelet-derived growth factor-BB and Ets-1 transcription factor negatively regulate transcription of multiple smooth muscle cell differentiation marker genes. Am J Physiol Heart Circ Physiol. 2004;286(6):H2042-51.

20. Thyberg J, Nilsson J, Palmberg L, Sjolund M. Adult human arterial smooth muscle cells in primary culture. Modulation from contractile to synthetic phenotype. Cell Tissue Res. 1985;239(1):69-74.

21. Palmberg L, Thyberg J. Uterine smooth muscle cells in primary culture. Alterations in fine structure, cytoskeletal organization and growth characteristics. Cell Tissue Res. 1986;246(2):253-62.

22. Frid MG, Shekhonin BV, Koteliansky VE, Glukhova MA. Phenotypic changes of human smooth muscle cells during development: late expression of heavy caldesmon and calponin. Dev Biol. 1992;153(2):185-93.

23. Glukhova MA, Frid MG, Shekhonin BV, Balabanov YV, Koteliansky VE. Expression of fibronectin variants in vascular and visceral smooth muscle cells in development. Dev Biol. 1990;141(1):193-202.

24. Vandekerckhove J, Weber K. At least six different actins are expressed in a higher mammal: an analysis based on the amino acid sequence of the amino-terminal tryptic peptide. $J \mathrm{Mol}$ Biol. 1978;126(4):783-802.

25. Owens GK. Regulation of differentiation of vascular smooth muscle cells. Physiol Rev. 1995;75(3):487-517.

26. Arnoldi R, Hiltbrunner A, Dugina V, Tille JC, Chaponnier C. Smooth muscle actin isoforms: a tug of war between contraction and compliance. Eur J Cell Biol. 2013;92(6-7):187-200.

27. Wang Z, Wang DZ, Pipes GC, Olson EN. Myocardin is a master regulator of smooth muscle gene expression. Proc Natl Acad Sci U S A. 2003;100(12):7129-34.

28. Du KL, Ip HS, Li J, Chen M, Dandre F, Yu W, et al. Myocardin is a critical serum response factor cofactor in the transcriptional program regulating smooth muscle cell differentiation. $\mathrm{Mol}$ Cell Biol. 2003;23(7):2425-37.

29. Wang Z, Wang DZ, Hockemeyer D, McAnally J, Nordheim A, Olson EN. Myocardin and ternary complex factors compete for SRF to control smooth muscle gene expression. Nature. 2004;428(6979):185-9.

30. Huang J, Wang T, Wright AC, Yang J, Zhou S, Li L, et al. Myocardin is required for maintenance of vascular and visceral smooth muscle homeostasis during postnatal development. Proc Natl Acad Sci U S A. 2015;112(14):4447-52.

31. Song J, Rolfe BE, Hayward IP, Campbell GR, Campbell JH. Reorganization of structural proteins in vascular smooth muscle cells grown in collagen gel and basement membrane matrices (Matrigel): a comparison with their in situ counterparts. J Struct Biol. 2001;133(1):43-54.

32. Thyberg J, Hultgardh-Nilsson A. Fibronectin and the basement membrane components laminin and collagen type IV influence the phenotypic properties of subcultured rat aortic smooth muscle cells differently. Cell Tissue Res. 1994;276(2):263-71.

33. Li X, Tsai P, Wieder ED, Kribben A, Van Putten V, Schrier RW, et al. Vascular smooth muscle cells grown on Matrigel. A model of the contractile phenotype with decreased activation of mitogen-activated protein kinase. J Biol Chem. 1994;269(30):19653-8.

34. Hedin U, Bottger BA, Forsberg E, Johansson S, Thyberg J. Diverse effects of fibronectin and laminin on phenotypic properties of cultured arterial smooth muscle cells. J Cell Biol. 1988;107(1):307-19.

35. Kleinman HK, Martin GR. Matrigel: basement membrane matrix with biological activity. Semin Cancer Biol. 2005;15(5):378-86.

36. Peyton SR, Raub CB, Keschrumrus VP, Putnam AJ. The use of poly(ethylene glycol) hydrogels to investigate the impact of ECM chemistry and mechanics on smooth muscle cells. Biomaterials. 2006;27(28):4881-93. 
37. Ma X, Wang $Y$, Stephens NL. Serum deprivation induces a unique hypercontractile phenotype of cultured smooth muscle cells. Am J Physiol. 1998;274(5 Pt 1):C1206-14.

38. Zimmermann O, Zwaka TP, Marx N, Torzewski M, Bucher A, Guilliard P, et al. Serum starvation and growth factor receptor expression in vascular smooth muscle cells. J Vasc Res. 2006;43(2):157-65.

39. Solodushko V, Khader HA, Fouty BW. Serum can overcome contact inhibition in confluent human pulmonary artery smooth muscle cells. PLoS One. 2013;8(8):e71490.

40. Hirst SJ, Twort CH, Lee TH. Differential effects of extracellular matrix proteins on human airway smooth muscle cell proliferation and phenotype. Am J Respir Cell Mol Biol. 2000;23(3):335-44.

41. Dekkers BG, Schaafsma D, Nelemans SA, Zaagsma J, Meurs H. Extracellular matrix proteins differentially regulate airway smooth muscle phenotype and function. Am J Physiol Lung Cell Mol Physiol. 2007;292(6):L1405-13.

42. Blank RS, Thompson MM, Owens GK. Cell cycle versus density dependence of smooth muscle alpha actin expression in cultured rat aortic smooth muscle cells. J Cell Biol. 1988;107(1):299-306.

43. Owens GK, Loeb A, Gordon D, Thompson MM. Expression of smooth muscle-specific alphaisoactin in cultured vascular smooth muscle cells: relationship between growth and cytodifferentiation. J Cell Biol. 1986;102(2):343-52. 



\section{CHAPTER 5}

Pancreatic tumor organoid-derived factors of patients with cachexia promote phenotypic modulation and proliferation of visceral smooth muscle cells 


\section{Abstract}

Introduction: Patients with pancreatic cancer often suffer from gastrointestinal symptoms which may be the consequence of underlying gastrointestinal motility problems. Although muscle loss in cachectic pancreatic cancer patients is most obvious in skeletal muscle, these clinical symptoms as well as our recent analysis of intestinal smooth muscle characteristics in cachectic patients suggest that cachexia manifests itself also in smooth muscle, a tissue responsible for contraction of the gastrointestinal tract. Pancreatic tumor organoids are in vitro 3D organ-like structures that retain key pathophysiological characteristics of the original tumor. We hypothesized that pancreatic tumor organoids from cachectic pancreatic cancer patients release factors that directly affect smooth muscle cell (SMC) contractile characteristics.

Methods: Human visceral SMCs were exposed to conditioned medium (CM, 50\% v/v) obtained from pancreatic tumor organoid cultures of cachectic $(n=2)$ and non-cachectic $(n=2)$ patients. Contractile proteins and markers of inflammation, muscle atrophy, and proliferation were evaluated by $\mathrm{QPCR}$ and Western blot. SMC proliferation and migration were monitored by live cell imaging. The Ki-67 positive cell fraction was determined in the intestinal smooth musculature of twenty-two pancreatic cancer patients with either a low or high L3 skeletal muscle mass index, as assessed by CT-scan analysis.

Results: CM from pancreatic tumor organoids of cachectic patients did not affect the expression of pro-inflammatory cytokines IL-1 $\beta$, IL- 6, IL- 8 , and MCP-1 or of the skeletal muscle E3-ubiquitin ligase Atrogin-1. However, exposure to organoid CM reduced protein levels of $\alpha$-smooth muscle actin ( $\alpha$-SMA) and smooth muscle protein 22- $\alpha$ (SM22 $\alpha$ ), two key proteins involved in SMC contraction. $\gamma$-smooth muscle actin expression was also significantly reduced. Loss of contractile SMC proteins was accompanied by a significantly reduced SMC doubling time (control: $36.2 \mathrm{~h}$ vs. organoid CM: $29.9 \mathrm{~h}, \mathrm{p}<0.001$ ) and increased mRNA expression of S100A4, a key protein involved in SMC proliferation. In line, a significantly higher percentage of Ki-67 positive nuclei was observed in the intestinal smooth musculature of pancreatic cancer patients with a low L3-SMI compared to those with a high L3-SMI (8.7 $\pm 2.1 \%$ vs $6.4 \pm 3.2 \%$ ( $p=0.047)$, respectively).

Conclusion: Pancreatic tumor organoids from cachectic patients secrete factors that diminish the contractile SMC phenotype and increase SMC proliferation. This may contribute to the frequently observed gastrointestinal motility problems in these patients. 


\section{Introduction}

Cachexia is a multifactorial wasting syndrome that is present in up to $80 \%$ of pancreatic cancer patients (1). It is defined as a complex metabolic syndrome that is predominantly characterized by the progressive loss of muscle mass, which is also referred to as sarcopenia. Cachexia is associated with reduced physical function, reduced tolerance to chemo- and radiotherapy, and a reduced quality of life (2). Moreover, it highly contributes to the poor prognosis of pancreatic cancer $(1,3)$.

The involuntary loss of body mass in cancer cachexia mainly concerns skeletal muscle, which accounts for $40 \%$ of total body weight. In response to systemic perturbations induced by the tumor (e.g. catabolic stimuli, elevated pro-inflammatory cytokines), muscle atrophy occurs as the result of a disturbed balance between anabolic and catabolic processes. Catabolic stimuli induce muscle-specific E3 ubiquitin ligases MuRF1 and Atrogin$1 / \mathrm{MAFbx}$ to bind to selective substrates to target them for ubiquitination and subsequent degradation by the $26 \mathrm{~S}$ proteasome (4). Similar alterations have recently been observed in the cardiac muscle of cachectic patients, suggesting that these pathways are also activated in cardiac muscle to induce muscle wasting $(5,6)$.

So far, it is not known whether the changes found in atrophying skeletal and cardiac muscle in response to tumor-derived factors also occur in smooth muscle, the third muscle compartment that fulfils a crucial role in human physiology. However, it is known that smooth muscle cells (SMCs) may also respond to atrophy-inducing stimuli by increasing ubiquitin ligase expression $(7,8)$. For example, Bdolah et al. showed that both MuRF1 and Atrogin-1/MAFbx are upregulated in the involuting uterine smooth muscle following delivery of the fetus, raising the possibility that activation of MuRF1 and Atrogin-1/MAFbx may be a common phenomenon in cells undergoing atrophy (7). Also, it has previously been shown that in response to inflammatory stimuli, SMCs can produce and release proinflammatory cytokines $(9,10)$. As systemic inflammation is suggested to play a key role in cancer cachexia-induced muscle wasting, smooth muscle cells may be targeted by these circulating inflammatory cytokines and play a role in sustaining it as well.

In contrast to skeletal and cardiac muscle cells that differentiate terminally, mature contractile SMCs retain remarkable plasticity as expressed by their ability to undergo socalled phenotypic modulation in response to injury and disease. Typical contractile SMCs are characterized by an elongated spindle-shaped morphology and high expression of a unique repertoire of contractile proteins. Loss of this contractile phenotype is accompanied by morphological alterations resulting in round-shaped SMCs, referred to as epithelioid or rhomboid, as well as increased proliferation rates, high migration rates, and increased synthesis of extracellular matrix (ECM) proteins (11).

Contractile SMCs are the force-producing cells essential for gut motor activity required to move nutrients through the gastrointestinal (GI)-tract (11). Diminished contractile SMC function results in GI-motility problems (12) that may contribute to nutritional-related complications in cancer cachexia. Indeed, Zhou and colleagues recently identified symptoms of early satiety, diarrhea, and constipation in cachectic cancer patients 
that could be secondary to gastrointestinal (GI) smooth muscle dysfunction (13). In further support of a role for smooth muscle in the pathophysiology of cachexia, we recently showed reduced expression of smoothelin, a key smooth muscle contractile protein, in the intestinal wall of pancreatic cancer patients with sarcopenia (Chapter 6). Also, pronounced collagen deposition around the myenteric plexus was observed in these patients, in line with increased ECM protein expression by synthetic SMCs. This may further disturb the generation of contractile forces that are regulated by the enteric nervous system.

In view of these recent data, we hypothesized that factors released by pancreatic tumor cells of cachectic patients induce phenotypic modulation of healthy contractile SMCs to their synthetic counterparts. Recent advances in modeling pancreatic cancer using threedimensional (3D) organoid cultures enable a novel approach to study this hypothesis (14, 15). Pancreatic tumor organoids self-organize into structures closely mimicking the tumor and accurately recapitulate many relevant aspects of disease progression in vitro and in vivo $(14,16)$. Importantly, cachexia severity of patients can be thoroughly assessed before establishing their organoid cultures, which is crucial to identify and better understand cachexia-inducing mechanisms in man. In the current study, we used our recently established pancreatic tumor organoid biobank (17) to obtain tumor-derived factors from two cachectic versus two non-cachectic patients. A validated human visceral SMC culture system was subsequently used to assess the effect of these tumor organoid-derived factors on the SMC phenotype (18). Lastly, we retrospectively assessed cellular proliferation in the intestinal smooth musculature of pancreatic cancer patients with either a low or high L3skelelal muscle index. 


\section{Materials and Methods}

\section{Human pancreatic tumor organoid culture}

Pancreatic tumor organoid cultures PANCO-9a, PANCO-11a, PANCO-12a, and PANCO-17a were previously generated and characterized in our laboratory (17). In short, for maintaining cultures, organoids were resuspended in ice-cold basement membrane extract (BME; Geltrex LDEV-Free Reduced Growth Factor Basement Membrane Matrix, Gibco, Cat. No. 1413202) and three $15 \mu \mathrm{L}$ droplets of Geltrex-cell suspension were allowed to solidify per well of a 24-wells culture plate (Eppendorf,) at $37^{\circ} \mathrm{C}$ for $30 \mathrm{~min}$. When the droplets were solidified, $500 \mu \mathrm{l}$ of organoid medium was added to each well (17). The plate was transferred to a humidified $37^{\circ} \mathrm{C} / 5 \% \mathrm{CO}_{2}$ incubator and medium was changed every 2-3 days. The organoids were passaged every 7-10 days.

\section{Collection of the pancreatic tumor organoid conditioned medium}

One day before passaging, organoid growth medium was replaced by basic culture medium consisting of DMEM/F12 supplemented with $1 \%(\mathrm{v} / \mathrm{v})$ HEPES and 1\% (v/v) Pen/Strep. Additional wells containing empty GX-droplets overlaid with DMEM/F12 were included for the collection of control medium. The medium was conditioned for 24h. After 24h, conditioned medium (CM) was collected and centrifuged at $350 \mathrm{xg}$ for $10 \mathrm{~min}$ at $4^{\circ} \mathrm{C}$. The supernatant was centrifuged for another $20 \mathrm{~min}$ at $2,000 \mathrm{xg}$ at $4^{\circ} \mathrm{C}$ and the resulting conditioned medium cleared from cellular debris was aliquoted and stored at $-80^{\circ} \mathrm{C}$. CM used for experiments has been collected between passage number 6 and 15 .

\section{Smooth muscle cell culture}

Human SMCs (ULTR cells) (19) were cultured in Dulbecco's Modified Eagle's Medium (DMEM) (Gibco, Cat. No. 42430) supplemented with $10 \%$ (v/v) fetal bovine serum (FBS) (Greiner Bio-one, Cat. No. 758093), 4 mM L-Glutamine, and antibiotics (100 units $/ \mathrm{mL}$ penicillin and $100 \mathrm{\mu g} / \mathrm{ml}$ streptomycin, Gibco). The cells were maintained at $37^{\circ} \mathrm{C}, 5 \% \mathrm{CO}_{2}$ in a humidified incubator.

For experiments, SMCs were cultured according to our previously published method that generates SMCs with a highly contractile phenotype (18). In short, SMCs were plated at a density of $1 \times 10^{4} \mathrm{cells} / \mathrm{cm}^{2}$ on Basement Membrane Matrix (BM) coated (Geltrex Reduced Growth Factor Basement Membrane Matrix, Gibco, Cat No. A14132) surfaces. When cells reached $>90 \%$ confluency after $48 \mathrm{~h}$, regular culture medium was replaced by advanced DMEM/F-12 supplemented with $2 \%(\mathrm{v} / \mathrm{v})$ FBS and antibiotics $(50$ units $/ \mathrm{mL}$ penicillin and $50 \mu \mathrm{g} / \mathrm{ml}$ streptomycin, Gibco). Medium was refreshed every three days. At day 6, SMCs were treated with $50 \%(\mathrm{v} / \mathrm{v})$ pancreatic tumor organoid-derived CM.

\section{Quantitative real-time $P C R$}

Total RNA was isolated using TRI Reagent (Sigma, St. Louis, MO) according to the manufacturer's instructions. RNA yield was measured with a DeNovix DS-11 
spectrophotometer and 375 ng RNA were reversed transcribed to cDNA using the SensiFast cDNA Synthesis Kit according to the manufacturer's protocol (Bioline $\mathrm{GmbH}$, Germany). Gene expression levels of SMC phenotype markers were quantified using a three-step PCR program followed by melting curve analysis using the LightCycler480 (Roche). cDNA was amplified with the SensiMix SYBR Hi-Rox kit (Bioline GmbH, Cat. No. QT605-05). Specific primer pairs for each gene were ordered from Sigma and are presented in table S1. Relative gene expression levels were derived from the LinRegPCR (version 2016.1) method (20) and normalized to the geometric average of two reference genes, cyclophylin A (CYPA) and beta2-microglobulin (B2M).

\section{Western blotting}

After contractile SMCs (day 6) were exposed to $50 \%(\mathrm{v} / \mathrm{v})$ pancreatic tumor organoidderived $\mathrm{CM}$ for $72 \mathrm{~h}$, cells were harvested in lysis buffer containing $10 \mathrm{mM}$ Tris, $100 \mathrm{mM}$ $\mathrm{NaCl}, 1 \mathrm{mM}$ EDTA, $1 \mathrm{mM}$ EGTA, $1 \%$ Triton X-100, 10\% glycerol, $0.1 \%(\mathrm{w} / \mathrm{v}) \mathrm{SDS}$, and $0.5 \%(\mathrm{v} / \mathrm{v})$ sodium deoxycholate supplemented with protease inhibitor cocktail tablets (Roche, Mannheim, Germany). The Western blot analysis was performed as described previously (18). In short, PVDF membranes were incubated overnight at $4^{\circ} \mathrm{C}$ with specific monoclonal primary antibodies directed against anti-smooth muscle myosin heavy chain (SM-MHC) (1:1000, Clone BT-562, Alfa Aesar, Cat. No. J64817AMJ), anti- $\alpha$-smooth muscle actin ( $\alpha-$ SMA) (1:1000, Clone 1A4, Dako, Cat. No. M0851), and anti-smooth muscle protein 22-alpha $(\mathrm{SM} 22 \alpha)(1: 1000)$ (the mouse anti-SM22 $\alpha$ hybridoma cell line, clone 3E11, was a kind gift from Dr. A. Chiavegato, University of Padua, Italy). After washing with TBS-Tween20 $(0.01 \%)$, the blots were probed for 1 hour with an appropriate peroxidase-conjugated secondary antibody (Vector Laboratories, CA, USA) and signals were visualized using SuperSignal West Pico chemiluminescent substrate (Thermo Scientific, Rockford, IL) according to the manufacturer's instructions. Images were obtained with a molecular imager (Amersham Imager 600, GE Healthcare Life Sciences) and total band intensity was quantified with ImageQuant TL software (v8.1.0.0, GE Healthcare Life Sciences).

\section{IncuCyte ${ }^{\mathrm{TM}}$ Cell Confluence Proliferation Assay}

SMCs were plated in 96-well cell culture plates (Eppendorf) at a density of $1 \times 10^{4} \mathrm{cells} / \mathrm{cm}^{2}$ on BM-coated surfaces. After 3 hours, the cells were attached to the surface and growth medium was replaced by $50 \%(\mathrm{v} / \mathrm{v})$ pancreatic tumor organoid derived-CM. Cell confluency was monitored by the IncuCyte ${ }^{\circledR}$ S3 Live-Cell analysis system (Sartorius). Phase-contrast images were captured every 2 hours using the 10x objective. Quantification of the occupied area (\% confluency) of all individual images over time was performed by using the integrated cell confluence analysis tool. The doubling time was calculated based on the linear part of the proliferation curve.

\section{IncuCyte ${ }^{\text {TM }}$ Scratch Wound Cell Migration Assay}

For the migration assay, SMCs were plated at a density of $1 \times 10^{4}$ cells $/ \mathrm{cm}^{2}$ on BM-coated IncuCyte $^{\circledR}$ ImageLock 96-well microplates. Subsequently, the SMCs were cultured as 
previously described (18). At day 6, homogeneous scratch wounds (700-800 $\mu \mathrm{m})$ were introduced by using the IncuCyte ${ }^{\circledR}$ WoundMaker tool (Essen Bioscience) according to the manufacturer's instructions. A cell-free zone was created within the confluent monolayer of SMCs in each well. Cell confluency within the cell-free zone was monitored by the IncuCyte ${ }^{\circledR}$ FLR system (2011A Rev2, Essen Bioscience). Phase-contrast images were captured every 2 hours using the 10x objective. The Relative Wound density v1.0 algorithm that is integrated in the software (2011A Rev2, EssenBioscience) was used to quantify the spatial cell density in the wound area relative to the spatial cell density outside of the wound area at every time point.

\section{Patient cohort}

Archived formalin-fixed paraffin-embedded (FFPE) jejunum tissue sections from twentytwo pancreatic cancer patients who underwent a Whipple procedure between 2009 and 2013 were obtained from the Pathology Department at the Maastricht University Medical Center (MUMC+), the Netherlands. The L3-skeletal muscle index (L3-SMI), which is a good estimate of total body skeletal muscle mass, was determined using pre-operative computed tomography (CT) scans at the level of the L3 vertebra. The scans were analysed as previously described (21). Sex-specific median cut-off values for L3-SMI were used to assign patients to either a high L3-SMI or low L3-SMI group. This study was approved by the local Medical Ethics Committee and was performed in accordance with the ethical standards laid down in the 1964 Declaration of Helsinki (22).

\section{Immunohistochemical staining and evaluation}

Paraffin-embedded jejunum tissue sections $(4 \mu \mathrm{m})$ were deparaffinized through xylene, treated with $0.6 \%(\mathrm{v} / \mathrm{v}) \mathrm{H}_{2} \mathrm{O}_{2}$ for 15 min to block endogenous peroxidase activity, and rehydrated through graded ethanol to water. Subsequently, the tissue sections were incubated for $20 \mathrm{~min}\left(90-95^{\circ} \mathrm{C}\right)$ with target retrieval solution according to the manufacturer's instruction (DAKO, \#S1699). The sections were rinsed with 1x Phosphatebuffered saline (PBS) and blocked for 30 min with 5\% BSA in 1xPBS to reduce background staining. Tissue sections were immunohistochemically stained with a monoclonal mouse anti-human Ki-67 antibody (1:200 dilution) (Clone MIB-1, Dako \#M7240). Subsequently, the tissue sections were washed and incubated with a rabbit anti-mouse IgG biotin labelled secondary antibody. The Vectastain Reagent was used to detect any biotinylated molecule. Peroxidase substrate solution (3,3'-Diaminobenzidine, DAB) (Dako) was used to visualize the presence of peroxidase enzyme. Sections were counterstained for 1 min with Hematoxylin (Merck, Darmstadt), washed, and then mounted with Entellan (Merck, Darmstadt).

Stained jejunal tissue slides were digitalized using the Ventana iScan HT (Version 1.1, Roche, Ventana Medical Systems, Inc.) using a 200x magnification. The scans were opened in Pannoramic Viewer software (version 1.15.4, 3DHISTECH, Ltd) for the selection of regions of interest (ROI). At least 5 representative ROls were selected in the transversal section of the circular smooth muscle layer (CL). 
Selected ROIs were converted to TIFF files and imported in QuPath (v0.1.2) (23). Positive and negative stained nuclei were counted and the percentage of positive Ki-67 stained nuclei was calculated for each individual ROI.

\section{Statistical analysis}

All data were obtained from three independent experiments performed in triplicate and were analyzed using IBM SPSS 25 for Microsoft Windows ${ }^{\circledR}$. Results are expressed as mean \pm SEM and statistical analyses were performed using the independent sample T-test to compare differences between two groups. In case of more than two groups, the One-way ANOVA test was used followed by Tukey's post-hoc testing. The Fisher's exact test was used for between-group comparisons of categorical variables. A $p$-value of $p<0.05$ was considered statistically significant. 


\section{Results}

Tumor organoid factors from non-cachectic pancreatic cancer patients induces upregulation of pro-inflammatory cytokines in human visceral SMCS

To investigate the effect of tumor-derived factors from pancreatic cancer patients on SMCs, we selected four recently generated pancreatic tumor organoid cultures from cachectic (PANCO-9a, PANCO-17a) and non-cachectic patients (PANCO-11a, PANCO-12a) (17). We first assessed whether tumor organoid factors from cachectic patients induced the expression of pro-inflammatory cytokines in SMCs (Fig. 1A). Unexpectedly, whereas tumor $\mathrm{CM}$ of non-cachectic patients induced the expression of interleukin-8 (IL-8) (2.5-fold, $\mathrm{p}=0.010$ ), this was not observed with CM of the cachectic patients. Similarly, IL-6 levels tended to be increased by the tumor secretome of non-cachectic patients (1.6-fold, $p=0.14$ ) but not by that of the cachectic patients. Mcp-1 and IL-1 $1 \beta$ expression were not affected by tumor factors from either cachectic or non-cachectic patients.

A

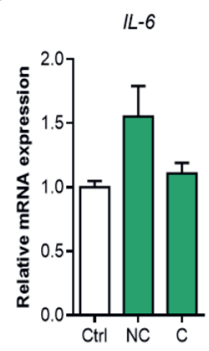

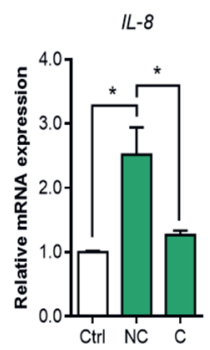

MCP-1

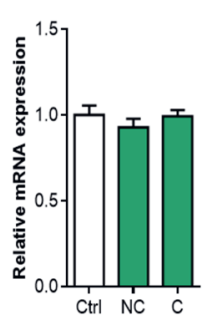

$I L-1 \beta$

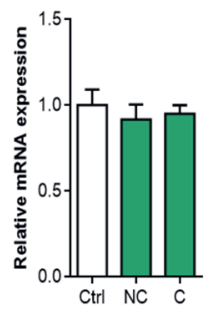

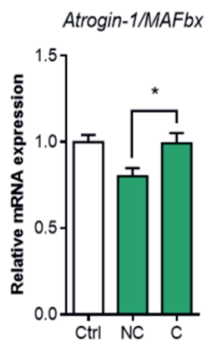

Figure 1: Tumor organoid factors from non-cachectic pancreatic cancer patients induce upregulation of pro-inflammatory cytokines by human SMCs. SMCs were cultured for 6 days on BM-coated surfaces and with $2 \%$ FBS to induce a contractile phenotype. At day 6 , SMCs were treated with $50 \%$ $(\mathrm{v} / \mathrm{v})$ pancreatic tumor organoid-derived CM. (A) mRNA expression levels of pro-inflammatory cytokines (IL-6, IL-8, MCP-1, and IL-16) and (B) E3-ubiquitin ligase Atrogin-1/MAFbx were determined after $48 \mathrm{~h}$. Data were normalized to CYPA and B2M reference genes. Relative expression levels are obtained from three independent experiments. Data are presented as mean $\pm S E M$. IL- 6 , interleukin6; IL-8, interleukin-8; MCP-1, monocyte chemoattractant protein- $1 ; I L-16$, interleukin-1B.

Atrogin-1/MAFbx is not upregulated in human visceral SMCs exposed to pancreatic tumor organoid derived $C M$

To investigate whether tumor organoid-derived factors induce activation of the ubiquitin proteasome system in SMCs like they do in skeletal muscle, we exposed contractile SMCs to pancreatic tumor organoid CM for 48h. Whereas MURF1 expression could not be detected, Atrogin-1/MAFbx was detectable but not increased compared to the control condition (Fig. 1B). This suggests that the ubiquitin proteasome pathway is not activated in response to pancreatic tumor organoid-derived factors from either cachectic or noncachectic patients. 
Tumor organoid factors from cachectic pancreatic cancer patients diminish SMC contractile protein levels

To investigate whether SMCs undergo phenotypic modulation in response to factors derived from pancreatic tumor cells of cachectic pancreatic cancer patients, contractile SMCs were exposed to tumor organoid CM for $72 \mathrm{~h}$ and levels of key contractile proteins (smooth muscle myosin heavy chain (SM-MHC), $\alpha$-smooth muscle actin ( $\alpha$-SMA), and smooth muscle protein 22-alpha (SM-22 $\alpha)$ ) were assessed by Western Blot. Although no differences in SM-MHC protein levels were observed, both $\alpha$-SMA (no-cachexia (NC): -1.7fold, $p<0.001$; cachexia (C): -1.6-fold, $p<0.001$ ) and SM22- $\alpha$ (NC: -2.3-fold, $p<0.001$; C: -3.2fold, $p<0.001$ ) protein levels were markedly decreased by the tumor organoid secretome of both cachectic and non-cachectic patients compared to the control condition (Fig. 2A-B). In line with this, mRNA expression of $\psi$-smooth muscle actin (ACTG2), the dominant actin isoform in visceral SMCs (24), was also significantly reduced compared to the control (NC: 1.6-fold, $\mathrm{p}<0.001 ; \mathrm{C}:-1.4$-fold, $\mathrm{p}<0.001$ ) (Fig. 2C). These results indicate that tumor-derived

A

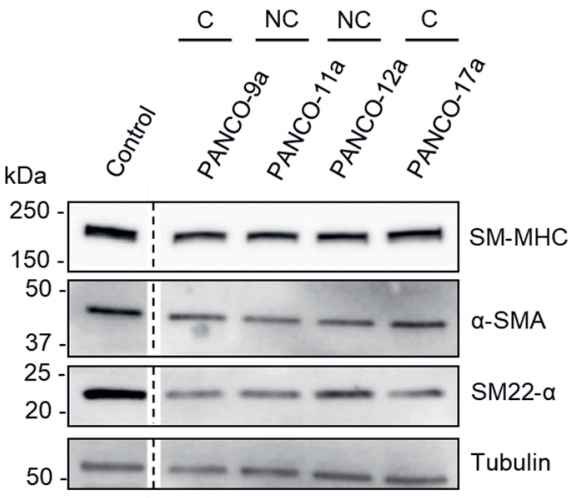

C

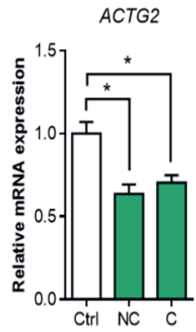

B
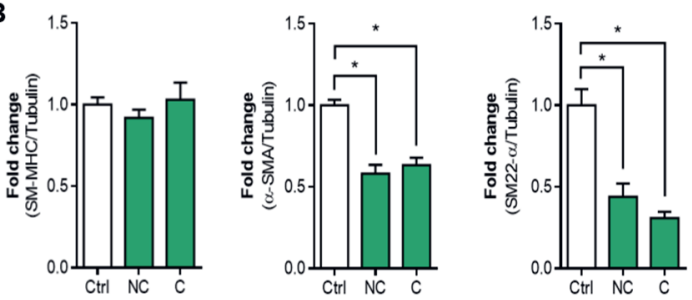

Figure 2: Tumor organoid factors from cachectic pancreatic cancer patients decrease SMC contractile proteins. Human SMCs were cultured for 6 days on BM-coated surfaces and with $2 \%$ FBS to induce high expression of contractile SMC markers. At day 6, SMCs were treated with $50 \%(\mathrm{v} / \mathrm{v})$ pancreatic tumor organoid-derived CM from cachectic (C: PANCO-9a and PANCO-17a) and noncachectic (NC: PANCO-11a and PANCO-12a) patients. (A) Protein levels of smooth muscle myosin heavy chain (SM-MHC), $\alpha$-smooth muscle actin ( $\alpha$-SMA), and smooth muscle protein 22- $\alpha$ (SM22 $\alpha$ ) were determined by Western Blot after $72 \mathrm{~h}$. (B) Protein levels were normalized to $\alpha$-Tubulin and control was set at 1. (C) mRNA expression of $\gamma$-smooth muscle actin (ACTG2) was determined after 48h. Data was normalized to CYPA and B2M references genes. Protein and relative gene expression levels were obtained from three independent experiments. Data are presented as mean \pm SEM. 
factors negatively affect the contractile phenotype of SMCs, consistent with phenotypic modulation to the synthetic phenotype.

Tumor organoid-derived factors induce proliferation but not migration of contractile human visceral SMCs

To confirm that tumor organoid-derived factors caused a shift towards the synthetic SMC phenotype, we next assessed key characteristics of synthetic SMCs, including their migration and proliferation rate, and the synthesis of ECM proteins. First, we performed a scratch wound assay on a monolayer of contractile SMCs in the presence or absence of tumor organoid CM. PDGF-BB (a well-known inducer of SMC proliferation and migration) and TGF- $\beta 1$ (a known inducer of the contractile SMC phenotype) were included as controls. Real-time monitoring of SMC migration revealed that the cell-free zone was covered with cells within $24 \mathrm{~h}$ after wounding in all conditions (Fig. 3A). As expected, the time needed for wound closure tended to be reduced in SMCs treated with PDGF-BB compared to the control condition (Fig. 3A-B). Opposite effects were observed with TGF- $\beta 1$. Similar to the effects observed with PDGF-BB, tumor organoid CM of cachectic patients seemed to accelerate the wound closure time as indicated by the wound width at the 12-hour timepoint (control: $405.6 \pm 37.5 \mu \mathrm{m}$ vs cachexia: $309.2 \pm 49.11, p=0.63$ ) (Fig. $3 A-B$ ). Nevertheless, the observed effects were not statistically significant.

To investigate whether SMCs synthesized increased levels of ECM proteins in response to pancreatic tumor organoid factors, the expression of genes encoding three major ECM proteins (collagen I (COL1A1), collagen III (COL3A1), and elastin (ELN)) was measured. Though COLBA1 expression tended to be increased after exposure to tumor organoid $\mathrm{CM}$ of cachectic patients compared to the control condition (1.4-fold, $p=0.16$ ), no significant differences in SMC COL1A1, COL3A1, or ELN mRNA expression were observed in SMCs exposed to tumor factors from cachectic or non-cachectic patients. (Fig. 3C).

Next, we assessed the proliferation rate of SMCs in response to tumor organoidderived factors. Cells were seeded at $\sim 20 \%$ confluency and exposed to tumor organoid CM or control medium, and confluency as a measure of proliferation was assessed over the next $72 \mathrm{~h}$. Interestingly, while monitoring the SMCs by live cell imaging, pronounced differences in their morphology were observed when cultured in tumor organoid CM (Fig. 4A). Whereas SMCs in control medium predominantly adopted elongated spindle shapes reminiscent of a contractile phenotype, SMCs exposed to pancreatic tumor organoid-derived factors of cachectic and non-cachectic patients were predominantly rhomboid-shaped. Moreover, we observed that the tumor organoid CM markedly accelerated coverage of the culture area by SMCs (Fig. 4B and Video S1-5). This observation was confirmed by the significantly reduced time contractile SMCs required to double in number in the exponential growth phase (40\%-80\% confluency) in the presence of tumor organoid CM from both cachectic and non-cachectic patients compared to the control (control: $36.2 \mathrm{~h}$ vs. no-cachexia $30.4 \mathrm{~h}$, $p=0.007$ and vs. cachexia: $30.0 \mathrm{~h}, \mathrm{p}=0.003$ ) (Fig. $4 \mathrm{C}$ ). As expected, TGF- $\beta 1$ significantly increased the doubling time (control: $36.2 \mathrm{~h}$ vs. TGF- $\beta 1$ : $47.4 \mathrm{~h}, \mathrm{p}<0.001$ ). To provide further support for the proliferative effect of tumor organoid factors on SMCs, we also assessed 
A

Control

on

Control

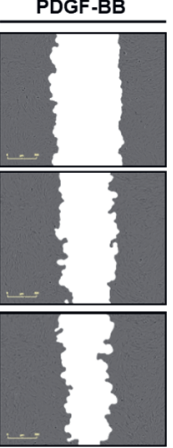

TGF-B1

PANCO-11a

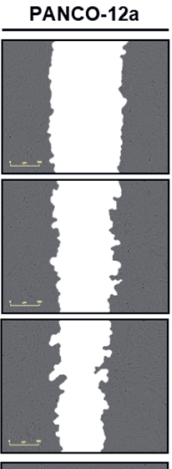

PANCO-9a

PANCO-17a

$6 \mathrm{~h}$

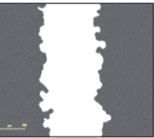

$12 \mathrm{~h}$
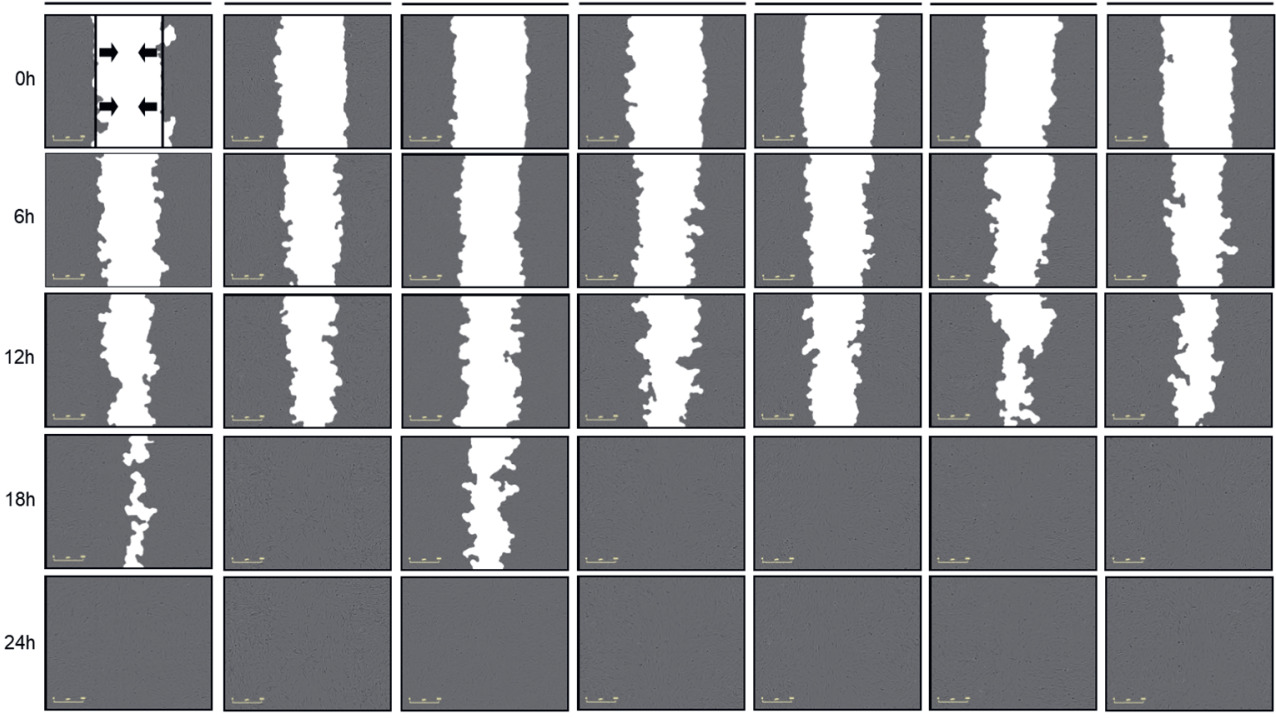

B

C
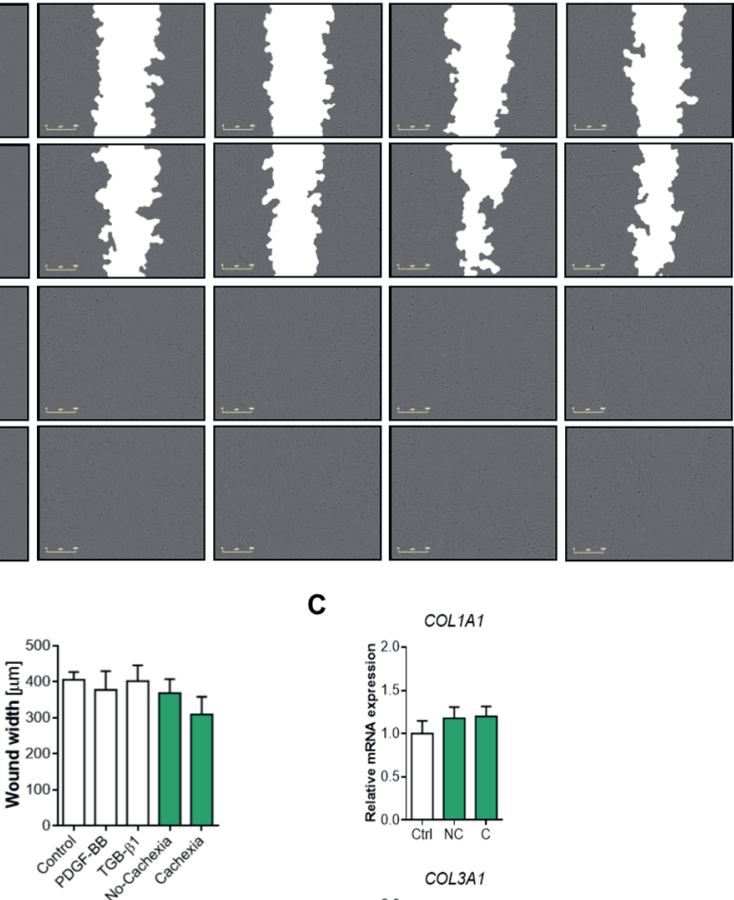

COLIA1
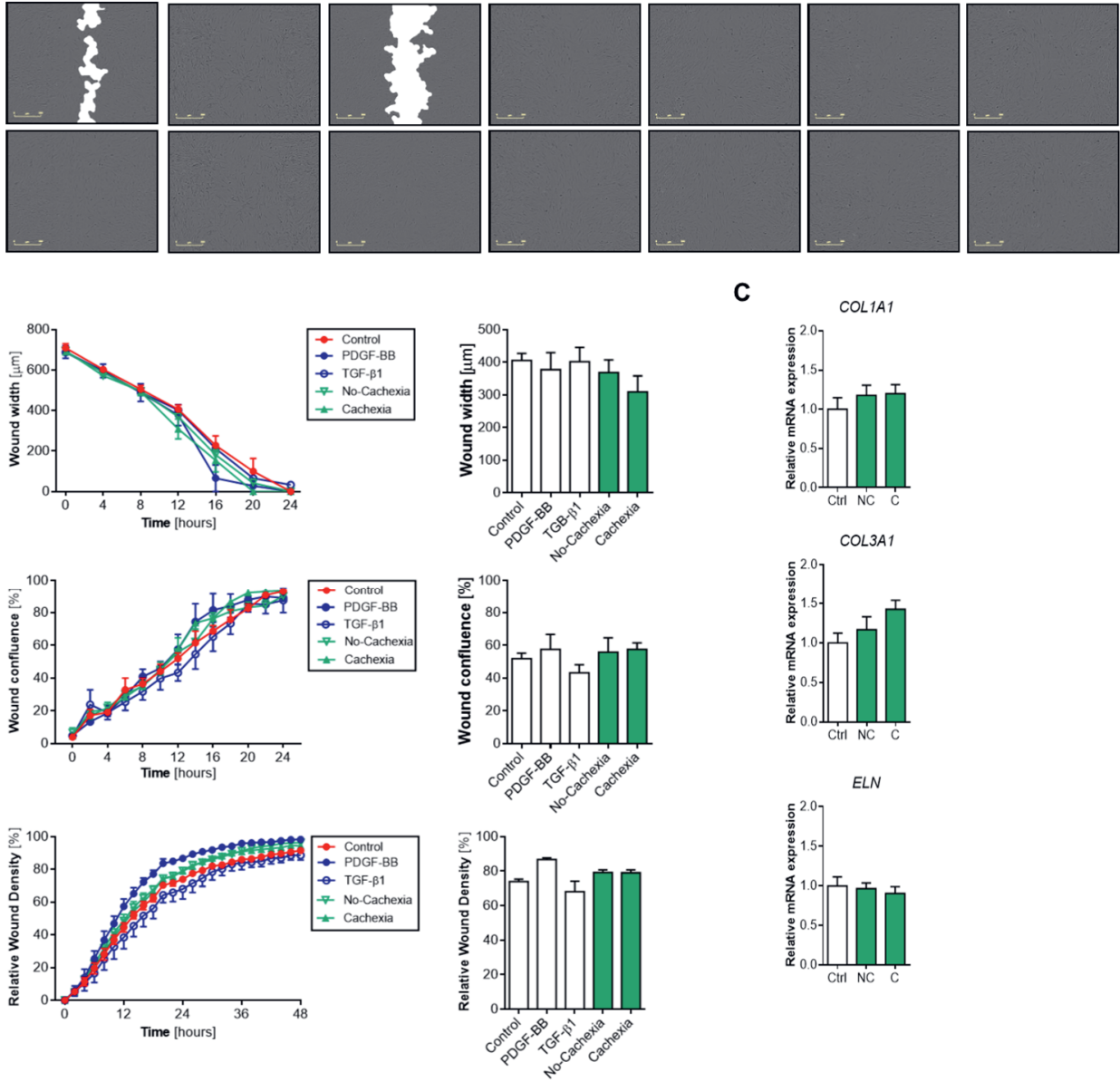

COL3A1

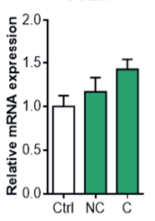

ELN

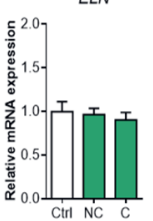

Figure 3: Tumor organoid-derived factors do not promote migration of contractile human SMCs. SMCs were cultured for 6 days on BM-coated surfaces and with $2 \%$ FBS to induce a contractile phenotype. At day 6, homogeneous scratch wounds were introduced. Immediately thereafter, contractile SMCs were treated with $50 \%(\mathrm{v} / \mathrm{v})$ pancreatic tumor organoid-derived CM from cachectic (C: PANCO-9a and PANCO-17a) and non-cachectic (NC: PANCO-11a and PANCO-12a) patients for 48h. Wound closure was measured in real-time over a period of $48 \mathrm{~h}$. (A) Representative phase-contrast images. Scale bar $=300 \mu \mathrm{m}$. Black arrows indicate the cell-free zone. (B) Migration of human visceral 
$<$ Previous page. SMCs is plotted as wound width $(\mu \mathrm{m})$, wound confluence $(\%)$, and relative wound density (\%) vs time (hours). The wound width represents the distance between wound boundaries over time, the wound confluence represents the cell confluence within the wound region over time, and the relative wound density represents the cell density in the wound area expressed relative to the cell density outside of the wound area over time (left panel). The wound width and the wound confluence at the $12 \mathrm{~h}$ time-point and the relative wound density at the $24 \mathrm{~h}$ time-point (right panel). Data are presented as mean \pm SEM; $n=3$ for each time point. Data were obtained from three independent experiments. (C) mRNA expression of genes encoding the ECM proteins collagen I (COL1A1), collagen III (COL3A1), and elastin (ELN) were determined after 48h. Data was normalized to CYPA and B2M references genes. Relative gene expression levels were obtained from three independent experiments. Data are presented as mean \pm SEM.

expression of $S 100 A 4$, a protein involved in $\operatorname{SMC}$ proliferation $(25,26)$. In line with the live cell imaging data, S100A4 mRNA expression was significantly increased by exposure of contractile SMCs to pancreatic tumor organoid factors from cachectic patients (1.4-fold, $\mathrm{p}=0.02$ ) (Fig. 4D). Together, these data show that pancreatic tumor organoid factors promote SMC proliferation, whereas migration and synthesis of ECM proteins are not affected.

Increased intestinal smooth muscle proliferation in pancreatic cancer patients is associated with low L3-SMI

To examine whether the induction of the proliferative SMC phenotype by tumor-derived factors observed in vitro could be validated in vivo, we retrospectively analyzed Ki-67 positive cell numbers in the intestinal smooth musculature of twenty-two pancreatic cancer patients. Patient characteristics are presented in table S2. Based on median sex-specific L3SMI cut-off values, patients were assigned to a high L3-SMI $(n=11)$ or a low L3-SMI $(n=11)$ group. A significantly higher percentage of Ki-67 positive nuclei was found in the intestinal smooth musculature of pancreatic cancer patients with a low L3-SMI compared to those patients with a high L3-SMI $(8.7 \pm 2.1 \%$ vs $6.4 \pm 3.2 \%$ ( $p=0.047)$, respectively) (Fig. 5A-B). These data imply a shift towards a proliferative, synthetic SMC phenotype in the intestinal musculature of pancreatic cancer patients with a low L3-SMI, which is a key characteristic of cancer cachexia. 
A
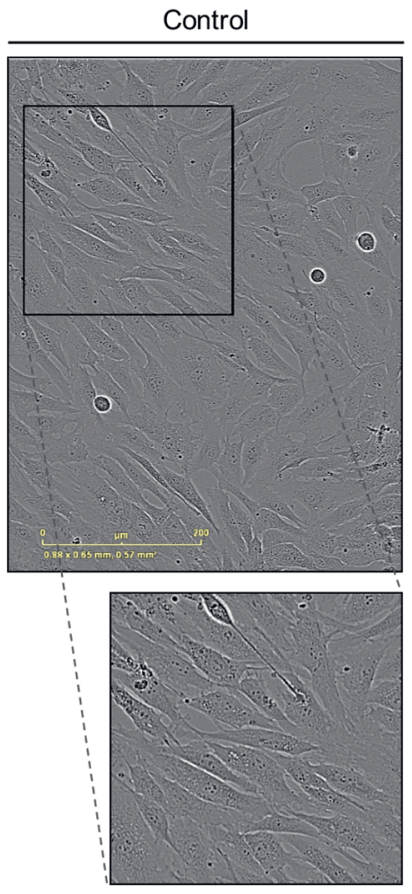

B

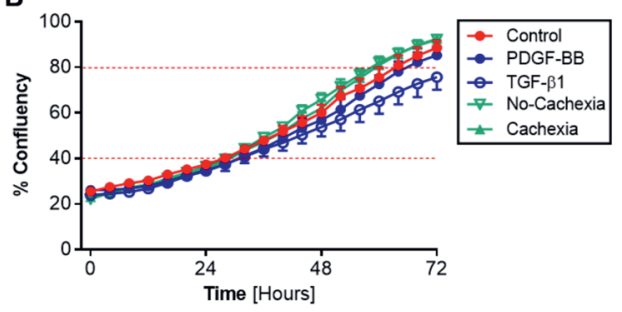

No-Cachexia
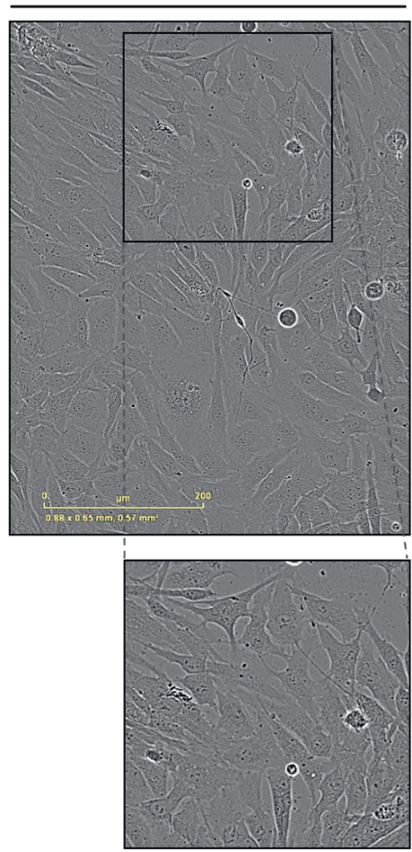

C

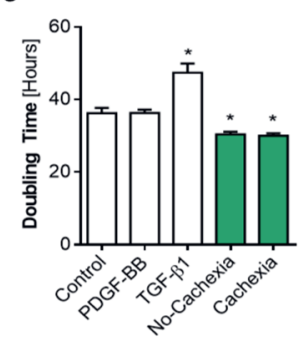

Cachexia
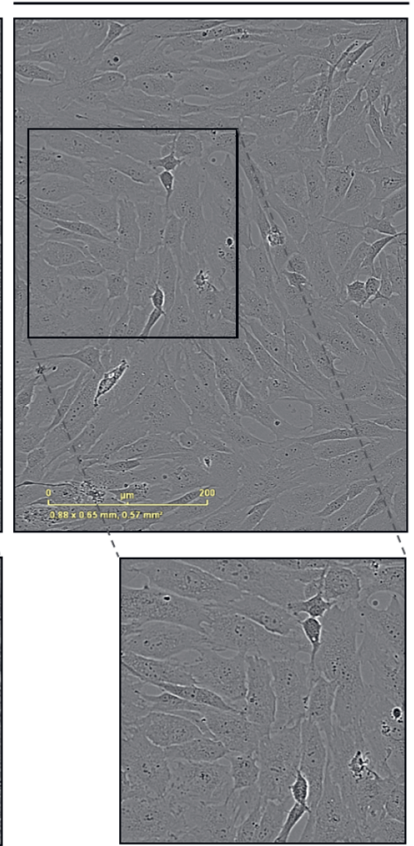

D

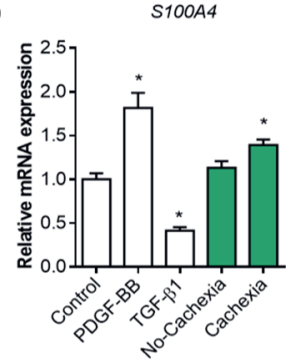

Figure 4: Tumor organoid-derived factors induce proliferation of contractile human SMCs. SMCS were treated with either control medium, PDGF-BB, TGF- $\beta 1$, or $50 \%(\mathrm{v} / \mathrm{v})$ pancreatic tumor organoid derived-CM from cachectic (PANCO-9a, PANCO-17a) and non-cachectic (PANCO-11a, PANCO-12a) patients. Cell confluency was monitored and phase-contrast images were captured every 2 hours. (A) Representative phase-contrast images after $72 \mathrm{~h}$. Scale bar $=200 \mu \mathrm{m}$. (B) Proliferation of human visceral SMCs is plotted as percentage (\%) confluency vs time (hours). (C) The doubling time was calculated for each individual group. The doubling time indicates the time SMCs require to double in number in the exponential growth phase (40-80\% confluency). (D) mRNA expression of S100A4 was determined in SMCs cultured for 6 days on BM-coated surfaces and with $2 \%$ FBS to induce contractile marker expression. These SMCs were treated for $48 \mathrm{~h}$ with control medium or $50 \%(\mathrm{v} / \mathrm{v})$ pancreatic tumor organoid-derived CM. Data was normalized to CYPA and B2M references genes. Data were obtained from three independent experiments. Data are presented as mean \pm SEM. 
A
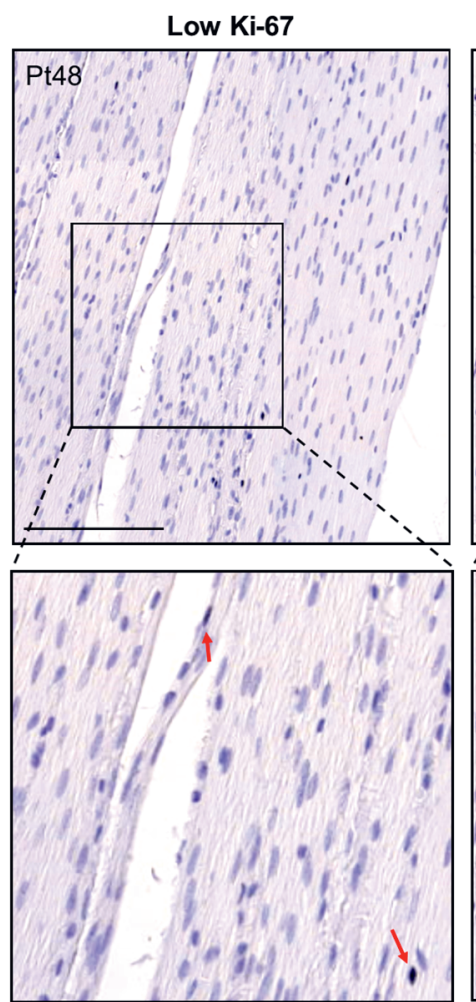

High Ki-67

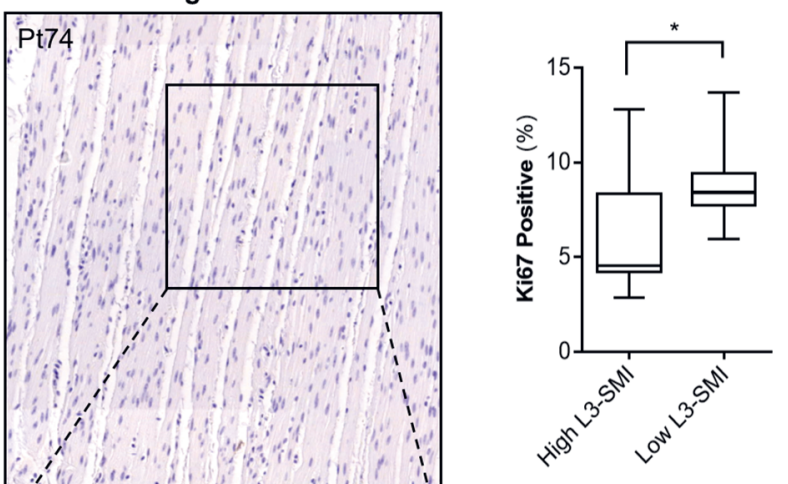

Figure 5: Increased intestinal smooth muscle proliferation in pancreatic cancer patients is associated with sarcopenia. Retrospective analysis of SMC proliferation in the intestinal smooth musculature of twenty-two pancreatic cancer patients. (A) Representative images of Ki-67 stained nuclei in the smooth musculature of patients with a high L3-SMI (left panel) or a low L3-SMI (right panel). Arrows indicate Ki-67 positive nuclei. Haematoxylin (blue/purple) was used as a nuclear stain. Scale bar $=200$ $\mu \mathrm{m}$. (B) The percentage of $\mathrm{Ki}-67$ positive nuclei is represented in a box plot graph. The boxes represent the interquartile range (IQR) in which the medians are indicated as a bar. Differences between patients with a high L3-SMI $(n=11)$ and a low L3-SMI $(n=11)$ were analyzed using the non-parametric MannWhitney U test, ${ }^{*} p<0.05$. 


\section{Discussion}

Cancer-induced muscle wasting is known to involve both striated muscle compartments, i.e. skeletal muscle and cardiac muscle $(5,6)$. So far, it is not known whether the aberrations found in atrophying skeletal and cardiac muscle in response to tumor-derived stimuli also occur in smooth muscle. Here, we showed that tumor organoid-derived factors from both cachectic and non-cachectic pancreatic cancer patients strongly reduce the expression of proteins involved in SMC contraction, reflecting loss of the SMC contractile phenotype. In parallel, proliferation of human visceral SMCs was accelerated, accompanied by increased expression of the proliferative marker S100A4 only after stimulation with tumor factors from cachectic patients. In line with these in vitro data, we detected an increased number of proliferating SMCs in the intestinal smooth musculature of pancreatic cancer patients with a low muscle mass. Overall, these data show that human contractile SMCs undergo phenotypic modulation in response to tumor-derived factors from pancreatic cancer patients, translating into a diminished contractile potential.

Muscle wasting in cancer cachexia is considered to be induced by tumor-derived factors that directly or indirectly activate intracellular signaling pathways disturbing cell homeostasis. In contrast to skeletal muscle, which is targeted for degradation by the muscle-specific E3 ubiquitin ligases MuRF1 and Atrogin1/MAFbx (4), no upregulation of either of these E3 ligases was detected in human visceral SMCs treated with tumor-organoid factors from cachectic pancreatic cancer patients. This is in line with our previous study, in which we revealed that tumor organoid factors from pancreatic cancer patients did not activate skeletal muscle atrophy signaling pathways in mature C2C12 myotubes (Chapter 3). A distinguishing feature of mature contractile SMCs is that they retain the capacity to reversibly modulate their phenotype in response to a variety of stimuli (27) and therefore, SMCs may respond differently to tumor-derived factors compared to skeletal muscle cells. In line with this concept, we showed that mature visceral SMCs expressed lower levels of contractile SMC-specific proteins after exposure to tumor organoid-derived factors, indicating that they lose their contractile phenotype. Loss of such SMC-specific proteins that are part of the contractile machinery of visceral SMCs can result in severe contractile impairment and consequent GI dysmotility $(11,28)$. In cancer patients, this may have potentially serious consequences including malnutrition, intolerance of oral anticancer agents, dehydration, and hospitalization (29).

The negative impact of tumor organoid factors on the contractile SMC phenotype was further supported by an increase in the SMC proliferation rate and upregulation of the proliferative marker S100A4. Our current understanding of non-vascular SMC proliferation under pathological conditions predominantly originates from studies investigating the role of inflammation on airway and intestinal SMCs. Both chronic airway diseases (e.g. asthma and chronic obstructive pulmonary disease) and inflammatory bowel diseases (e.g. Crohn's disease) are characterized by the presence of inflammation, which promotes SMC 
proliferation (30-35). In particular, the inflammatory cytokines tumor necrosis factor alpha (TNF- $\alpha$ ) and IL-1 $\beta$ have been shown to induce airway and intestinal SMC proliferation both in vitro and in vivo $(32-34,36)$. This may indicate that visceral SMCs, regardless of the location (i.e. wall of the urinary bladder, uterus, stomach, intestine, or airway), respond similarly to these pro-inflammatory cytokines that affect SMC phenotype and function. In line, we previously showed that pancreatic tumor organoids from cachectic and noncachectic pancreatic cancer patients express variable levels of known cachexia-associated cytokines, including IL-6, TNF- $\alpha$, IL-8, IL-1 $\alpha$, IL-1 $\beta$, Mcp-1, and LIF (17). Furthermore, increasing evidence also indicates that the enhanced catabolism experienced by cachectic cancer patients is mediated primarily by increases in pro-inflammatory cytokines, including TNF- $\alpha$, IL-1, and IL- 6 (37). This further supports the notion that pro-inflammatory cytokines may be responsible for the observed loss of the contractile phenotype and induction of SMC proliferation. Future studies should focus on the identification of these tumor-secreted cytokines and the potential causal effect of these identified factors on visceral SMCs.

Beyond the contractile and proliferative phenotype, SMCs can also participate in and coordinate the inflammatory response by the synthesis and secretion of several signaling molecules, such as pro-inflammatory cytokines, chemotaxis-associated molecules, and growth factors. Here, we showed that tumor organoid factors from non-cachectic patients significantly induced IL-8 expression and tended to increase IL-6 expression. Although unexpected, these data are in line with previous studies that revealed IL-1 $\beta$-induced upregulation and release of several pro-inflammatory cytokines, including IL-6, IL-8, and Mcp-1 in visceral SMCs (38-40). We previously showed that tumor organoids from noncachectic pancreatic cancer patients expressed higher IL-1 $\beta$ mRNA levels compared to tumor organoids from cachectic patients (17). These data suggest that tumor-secreted cytokines may directly induce intracellular changes in SMCs, resulting in the synthesis and secretion of pro-inflammatory cytokines. These alterations can contribute to a persistent systemic inflammatory condition.

Whereas the walls of many visceral organs are surrounded by well-developed smooth muscle layers that enable endured constriction or dilation, the role and behavior of visceral smooth muscle under pathological conditions remains poorly investigated. This is particularly due to difficulties in obtaining human visceral smooth muscle tissue of the organ of interest and the establishment of primary visceral SMC lines that retain a contractile phenotype in culture. In vitro, SMCs are known to modulate uncontrollably toward a synthetic phenotype under standard culture conditions that lack the physiological microenvironment that normally keeps the cells in a contractile phenotype (41). Moreover, the phenotype and function of SMCs is highly sensitive to environmental stimuli. To surpass these limitations, we used our recently developed human visceral SMC culture model in which SMCs can be kept in either a highly contractile state or modulated toward the synthetic phenotype in a controlled way (18). This model greatly contributed to our current observations and the relevance of our findings with respect to the human in vivo situation. Nevertheless, contractile functionality cannot be assessed in this in vitro model and also 
data regarding the functionality of the Gl-tract are lacking in our validation study. This should be integrated in future studies to be able to further explore a possible link between cachexia and smooth muscle dysfunction and the possible consequences, including malnutrition.

In conclusion, our data reveal that tumor-derived factors from cachectic patients not only affect skeletal muscle but also induce SMC phenotypic modulation. Loss of the contractile phenotype and increased SMC proliferation is known to impair the contractile functionality of the intestinal smooth musculature, and it may therefore underlie the frequently reported Gl-symptoms in cachectic cancer patients. 


\section{References}

1. Baracos VE, Martin L, Korc M, Guttridge DC, Fearon KCH. Cancer-associated cachexia. Nat Rev Dis Primers. 2018;4:17105.

2. Fearon K, Strasser F, Anker SD, Bosaeus I, Bruera E, Fainsinger RL, et al. Definition and classification of cancer cachexia: an international consensus. Lancet Oncol. 2011;12(5):48995.

3. Kleeff J, Korc M, Apte M, La Vecchia C, Johnson CD, Biankin AV, et al. Pancreatic cancer. Nat Rev Dis Primers. 2016;2:16022.

4. Bodine SC, Baehr LM. Skeletal muscle atrophy and the E3 ubiquitin ligases MuRF1 and MAFbx/atrogin-1. Am J Physiol Endocrinol Metab. 2014;307(6):E469-84.

5. Barkhudaryan A, Scherbakov N, Springer J, Doehner W. Cardiac muscle wasting in individuals with cancer cachexia. ESC Heart Fail. 2017;4(4):458-67.

6. Springer J, Tschirner A, Haghikia A, von Haehling S, Lal H, Grzesiak A, et al. Prevention of liver cancer cachexia-induced cardiac wasting and heart failure. Eur Heart J. 2014;35(14):932-41.

7. Bdolah Y, Segal A, Tanksale P, Karumanchi SA, Lecker SH. Atrophy-related ubiquitin ligases atrogin-1 and MuRF-1 are associated with uterine smooth muscle involution in the postpartum period. Am J Physiol Regul Integr Comp Physiol. 2007;292(2):R971-6.

8. Nakashima K, Ishida A, Katsumata M. Atrogin-1/MAFbx, a muscle-specific ubiquitin ligase, is highly expressed in the smooth muscle of the chicken gizzard. Biosci Biotechnol Biochem. 2013;77(5):1092-5.

9. Bouchelouche K, Alvarez S, Horn T, Nordling J, Bouchelouche P. Human detrusor smooth muscle cells release interleukin-6, interleukin-8, and RANTES in response to proinflammatory cytokines interleukin-1beta and tumor necrosis factor-alpha. Urology. 2006;67(1):214-9.

10. Ng EK, Panesar N, Longo WE, Shapiro MJ, Kaminski DL, Tolman KC, et al. Human intestinal epithelial and smooth muscle cells are potent producers of IL-6. Mediators Inflamm. 2003;12(1):3-8.

11. Scirocco A, Matarrese P, Carabotti M, Ascione B, Malorni W, Severi C. Cellular and Molecular Mechanisms of Phenotypic Switch in Gastrointestinal Smooth Muscle. J Cell Physiol. 2016;231(2):295-302.

12. Sanders KM, Koh SD, Ro S, Ward SM. Regulation of gastrointestinal motility--insights from smooth muscle biology. Nat Rev Gastroenterol Hepatol. 2012;9(11):633-45.

13. Zhou T, Yang K, Thapa S, Liu H, Wang B, Yu S. Differences in Symptom Burden Among Cancer Patients With Different Stages of Cachexia. J Pain Symptom Manage. 2017;53(5):919-26.

14. Boj SF, Hwang Cl, Baker LA, Chio, II, Engle DD, Corbo V, et al. Organoid models of human and mouse ductal pancreatic cancer. Cell. 2015;160(1-2):324-38.

15. Broutier L, Andersson-Rolf A, Hindley CJ, Boj SF, Clevers H, Koo BK, et al. Culture and establishment of self-renewing human and mouse adult liver and pancreas 3D organoids and their genetic manipulation. Nat Protoc. 2016;11(9):1724-43.

16. Clevers H. Modeling Development and Disease with Organoids. Cell. 2016;165(7):1586-97.

17. Vaes RDW, van Dijk DPJ, Welbers TTJ, Blok MJ, Aberle MR, Heij L, et al. Generation and initial characterization of novel tumour organoid models to study human pancreatic cancerinduced cachexia. J Cachexia Sarcopenia Muscle. 2020.

18. Vaes RDW, van den Berk L, Boonen B, van Dijk DPJ, Olde Damink SWM, Rensen SS. A novel human cell culture model to study visceral smooth muscle phenotypic modulation in health and disease. Am J Physiol Cell Physiol. 2018;315(4):C598-C607.

19. Perez-Reyes N, Halbert CL, Smith PP, Benditt EP, McDougall JK. Immortalization of primary human smooth muscle cells. Proc Natl Acad Sci U S A. 1992;89(4):1224-8. 
20. Ruijter JM, Ramakers C, Hoogaars WM, Karlen Y, Bakker O, van den Hoff MJ, et al. Amplification efficiency: linking baseline and bias in the analysis of quantitative PCR data. Nucleic Acids Res. 2009;37(6):e45.

21. van Dijk DP, Bakens MJ, Coolsen MM, Rensen SS, van Dam RM, Bours MJ, et al. Low skeletal muscle radiation attenuation and visceral adiposity are associated with overall survival and surgical site infections in patients with pancreatic cancer. J Cachexia Sarcopenia Muscle. 2017;8(2):317-26.

22. Rickham PP. Human Experimentation. Code of Ethics of the World Medical Association. Declaration of Helsinki. Br Med J. 1964;2(5402):177.

23. Bankhead P, Loughrey MB, Fernandez JA, Dombrowski Y, McArt DG, Dunne PD, et al. QuPath: Open source software for digital pathology image analysis. Sci Rep. 2017;7(1):16878.

24. Arnoldi R, Hiltbrunner A, Dugina V, Tille JC, Chaponnier C. Smooth muscle actin isoforms: a tug of war between contraction and compliance. Eur J Cell Biol. 2013;92(6-7):187-200.

25. Brisset AC, Hao H, Camenzind E, Bacchetta M, Geinoz A, Sanchez JC, et al. Intimal smooth muscle cells of porcine and human coronary artery express S100A4, a marker of the rhomboid phenotype in vitro. Circ Res. 2007;100(7):1055-62.

26. Chaabane C, Heizmann CW, Bochaton-Piallat ML. Extracellular S100A4 induces smooth muscle cell phenotypic transition mediated by RAGE. Biochim Biophys Acta. 2015;1853(9):2144-57.

27. Beamish JA, He P, Kottke-Marchant K, Marchant RE. Molecular regulation of contractile smooth muscle cell phenotype: implications for vascular tissue engineering. Tissue Eng Part B Rev. 2010;16(5):467-91.

28. Niessen P, Rensen S, van Deursen J, De Man J, De Laet A, Vanderwinden JM, et al. Smoothelin-a is essential for functional intestinal smooth muscle contractility in mice. Gastroenterology. 2005;129(5):1592-601.

29. Donthireddy KR, Ailawadhi S, Nasser E, Schiff MD, Nwogu CE, Nava HR, et al. Malignant gastroparesis: pathogenesis and management of an underrecognized disorder. J Support Oncol. 2007;5(8):355-63.

30. Khan MA. Inflammation signals airway smooth muscle cell proliferation in asthma pathogenesis. Multidiscip Respir Med. 2013;8(1):11.

31. Nair DG, Han TY, Lourenssen S, Blennerhassett MG. Proliferation modulates intestinal smooth muscle phenotype in vitro and in colitis in vivo. Am J Physiol Gastrointest Liver Physiol. 2011;300(5):G903-13.

32. Nair DG, Miller KG, Lourenssen SR, Blennerhassett MG. Inflammatory cytokines promote growth of intestinal smooth muscle cells by induced expression of PDGF-Rbeta. J Cell Mol Med. 2014;18(3):444-54.

33. Owens MW, Grisham MB. Cytokines increase proliferation of human intestinal smooth muscle cells: possible role in inflammation-induced stricture formation. Inflammation. 1993;17(4):481-7.

34. Stamatiou R, Paraskeva E, Gourgoulianis K, Molyvdas PA, Hatziefthimiou A. Cytokines and growth factors promote airway smooth muscle cell proliferation. ISRN Inflamm. 2012;2012:731472.

35. Yan F, Gao H, Zhao H, Bhatia M, Zeng Y. Roles of airway smooth muscle dysfunction in chronic obstructive pulmonary disease. J Transl Med. 2018;16(1):262.

36. Stanzel RD, Lourenssen S, Nair DG, Blennerhassett MG. Mitogenic factors promoting intestinal smooth muscle cell proliferation. Am J Physiol Cell Physiol. 2010;299(4):C805-17.

37. Cole CL, Kleckner IR, Jatoi A, Schwarz EM, Dunne RF. The Role of Systemic Inflammation in Cancer-Associated Muscle Wasting and Rationale for Exercise as a Therapeutic Intervention. JCSM Clin Rep. 2018;3(2). 
38. Lappas M. The IL-1beta signalling pathway and its role in regulating pro-inflammatory and pro-labour mediators in human primary myometrial cells. Reprod Biol. 2017;17(4):333-40.

39. Liao Z, Xiao HT, Zhang Y, Tong RS, Zhang $\mathrm{J}$, Bian Y, et al. IL-1beta: a key modulator in asthmatic airway smooth muscle hyper-reactivity. Expert Rev Respir Med. 2015;9(4):429-36.

40. Parpaleix A, Amsellem V, Houssaini A, Abid S, Breau M, Marcos E, et al. Role of interleukin1 receptor $1 /$ MyD88 signalling in the development and progression of pulmonary hypertension. Eur Respir J. 2016;48(2):470-83.

41. Chamley-Campbell J, Campbell GR, Ross R. The smooth muscle cell in culture. Physiol Rev. 1979;59(1):1-61. 


\section{Supplemental tables}

Table S1: qRT-PCR primers

\begin{tabular}{|c|c|c|}
\hline Gene & Full name & Primer Sequence \\
\hline \multirow[t]{2}{*}{ ACTG2 } & $\gamma$-smooth muscle actin & FW: 5' - CGCCCTCGCCACCAG - 3' \\
\hline & & RV: 5' - CCTTGGGATTTAGGGGAGCC - 3' \\
\hline \multirow[t]{2}{*}{ ATROGIN } & Atrogin-1/Muscle atrophy F-box & FW: 5' - AGTTTCGTGAGCGACCTCAG - 3' \\
\hline & protein & RV: 5' - TGGTGGAAATACTGAGTTTTTGGT - 3' \\
\hline \multirow[t]{2}{*}{ COL1A1 } & Collagen Type I Alpha 1 Chain & FW: 5’ - GGTCAGATGGGCCCCCG - 3' \\
\hline & & RV: 5' - GCACCATCATTTCCACGAGC - 3' \\
\hline \multirow[t]{2}{*}{ COL3A1 } & Collagen Type III Alpha 1 Chain & FW: 5' - GAAAGATGGCCCAAGGGGTC - 3' \\
\hline & & RV: 5' - TATACCTGGAAGTCCGGGGG - 3' \\
\hline \multirow[t]{2}{*}{ ELN } & Elastin & FW: 5' - TGTCTGCAGCCCCTTCTGTG - 3' \\
\hline & & RV: 5' - GGCACTTTTCCCAGGCTTCA - 3' \\
\hline \multirow[t]{2}{*}{ IL1B } & Interleukin 1 beta & FW: 5' - CTGAGCTCGCCAGTGAAATG - 3' \\
\hline & & RV: 5' - TTTAAGGGCCATCAGCTTCAAA - 3' \\
\hline \multirow[t]{2}{*}{ IL6 } & Interleukin 6 & FW: 5' - TACCCCCAGGAGAAGATTCC - 3' \\
\hline & & RV: 5' - TTTCAGCCATCTTTGGAAGG - 3' \\
\hline \multirow[t]{2}{*}{ IL8 } & Interleukin 8 & FW: 5' - CTGGCCGTGGCTCTCTTG - 3' \\
\hline & & RV: 5' - TTAGCACTCCTTGGCAAAACTG - 3' \\
\hline \multirow[t]{2}{*}{ MCP1 } & Monocyte chemotactic protein 1 & FW: 5' - TCACCTGCTGTTATAACTTCAC - 3' \\
\hline & & RV: 5' - CAATGGTCTTGAAGATCACAG - 3' \\
\hline \multirow[t]{2}{*}{ S100A4 } & S100 Calcium Binding Protein A4 & FW: 5' - TCTTGGTTTGATCCTGACTGCT - 3' \\
\hline & & RV: 5' - GCCCGAGTACTTGTGGAAGG - 3' \\
\hline \multirow[t]{2}{*}{$B 2 M$} & Beta-2-microglobulin & FW: 5' - TCCATCCGACATTGAAGTTG - 3' \\
\hline & & RV: 5' - CGGCAGGCATACTCATCTTT - 3' \\
\hline \multirow[t]{2}{*}{ CYPA } & Cyclophylin A & FW: 5' - CTCGAATAAGTTTGACTTGTGTTT - 3' \\
\hline & & RV: 5' - CTAGGCATGGGAGGGAACA - 3' \\
\hline
\end{tabular}


Table S2: Patient characteristics

\begin{tabular}{lllll}
\hline & $\begin{array}{l}\text { Total } \\
(N=22)\end{array}$ & $\begin{array}{l}\text { High L3-SMI } \\
(N=11)\end{array}$ & $\begin{array}{l}\text { Low L3-SMI } \\
(N=11)\end{array}$ & $p$-value \\
\hline Male $(\mathrm{n}, \%)$ & $10(45.5 \%)$ & $5(45.5 \%)$ & $5(45.5 \%)$ & $\mathrm{p}=1.00$ \\
Age (years) & $67.8( \pm 8.5)$ & $67.8( \pm 7.2)$ & $67.8( \pm 10.0)$ & $\mathrm{p}=1.00$ \\
BMI $\left(\mathrm{kg} / \mathrm{m}^{2}\right)$ & $23.9( \pm 2.9)$ & $25.3( \pm 3.0)$ & $22.5( \pm 1.9)$ & $\mathrm{p}=0.015$ \\
Weight loss ${ }^{*}(\%)$ & $9.4( \pm 7.4)$ & $8.2( \pm 7.0)$ & $10.7( \pm 7.9)$ & $\mathrm{p}=0.44$ \\
MUST score $\geq 2(\mathrm{n}, \%)$ & $8(36.4 \%)^{\#}$ & $3(30.0 \%)^{\#}$ & $5(45.5 \%)$ & $\mathrm{p}=0.66$ \\
L3-Skeletal muscle index & & & & \\
$\left(\mathrm{cm}^{2} / \mathrm{m}^{2}\right)$ & & & & \\
$\quad$ Male & & & & \\
$\quad$ Female & $49.3( \pm 4.3)$ & $52.4( \pm 3.6)$ & $46.1( \pm 1.9)$ & $\mathrm{p}=0.0084$ \\
\hline
\end{tabular}

" Weight loss over the past 6 months; ${ }^{*}$ missing data, $n=1$ 


\section{Supplemental videos}

Video S1: Human visceral SMC proliferation - Control. Human visceral SMCs were cultured for 3 days on BM-coated surfaces in control medium containing $2 \%(\mathrm{v} / \mathrm{v})$ FBS and $50 \%(\mathrm{v} / \mathrm{v}) \mathrm{DMEM} / \mathrm{F} 12$. Scale bar $=400 \mu \mathrm{m}$.

Video S2: Human visceral SMC proliferation - PANCO-9a CM. Human visceral SMCs were cultured for 3 days on BM-coated surfaces in medium containing 2\% (v/v) FBS and 50\% (v/v) PANCO-9a-derived CM. Scale bar $=400 \mu \mathrm{m}$.

S3: Human visceral SMC proliferation - PANCO-11a CM. Human visceral SMCs were cultured for 3 days on BM-coated surfaces in medium containing 2\% (v/v) FBS and 50\% (v/v) PANCO-11aderived $\mathrm{CM}$. Scale bar $=400 \mu \mathrm{m}$.

Video S4: Human visceral SMC proliferation - PANCO-12a CM. Human visceral SMCs were cultured for 3 days on BM-coated surfaces in medium containing 2\% (v/v) FBS and 50\% (v/v) PANCO-12aderived $\mathrm{CM}$. Scale bar $=400 \mu \mathrm{m}$.

Video S5: Human visceral SMC proliferation - PANCO-17a CM. Human visceral SMCs were cultured for 3 days on BM-coated surfaces in medium containing 2\% (v/v) FBS and 50\% (v/v) PANCO-17aderived $\mathrm{CM}$. Scale bar $=400 \mu \mathrm{m}$. 


\section{CHAPTER 6}

\section{Intestinal smooth muscle aberrations in pancreatic cancer patients with sarcopenia}

Rianne D.W. Vaes, Tessa T.J. Welbers, David P.J. van Dijk, Dorit Rennspiess, Axel zur Hausen, Steven W.M. Olde Damink, Sander S. Rensen

JCSM Rapid Communications (2021) 


\section{Abstract}

Background: Cancer cachexia is characterized by impaired function of skeletal and cardiac muscle. Smooth muscle is abundantly present in the body and critical for the function of the gastrointestinal tract. Given the frequently reported gastrointestinal symptoms in cancer patients, we hypothesized that the smooth musculature could be compromised in cancer patients with sarcopenia.

Methods: Full-thickness jejunal tissue sections from 57 pancreatic cancer patients were analyzed by picrosirius red stains and immunohistochemistry for $\alpha$-smooth muscle actin ( $\alpha$ SMA), smoothelin, and CD117 (c-kit). Muscle wall thickness, contractile marker expression, and collagen deposition were quantified. Patients were assigned to a sarcopenia or nonsarcopenia group based on their skeletal muscle index (SMI).

Results: Intestinal smooth muscle wall thickness did not differ between the sarcopenia and non-sarcopenia group $(1661 \pm 125.0$ vs. $1439 \pm 93.5 \mu \mathrm{m}, \mathrm{p}=0.41)$. Whereas $\alpha$-SMA staining intensity was similar in both groups, staining intensity of smoothelin, a key marker of the contractile smooth muscle cell phenotype, was reduced $(143.0 \pm 22.6$ vs. $125.4 \pm 29.3$ arbitrary units, $p=0.02$ ) in sarcopenic patients. The distribution of $C D 117^{+}$interstitial cells of Cajal was similar in both groups, but pronounced collagen deposition around the myenteric plexus was more often observed in patients with sarcopenia $(p=0.04)$.

Conclusions: These data suggest that cancer cachexia is not only associated with skeletal and cardiac muscle wasting, but also affects the intestinal smooth musculature. Reduced contractile smooth muscle marker expression and fibrosis around the myenteric plexus suggest that both contractile function of smooth muscle cells and regulation of their contractile functionality could be compromised. 


\section{Introduction}

Cancer cachexia is a multifactorial metabolic syndrome of severe weight loss and muscle wasting, which is estimated to affect more than half of all cancer patients (1). Although cachexia is particularly highly prevalent in end-stage disease, around $70-80 \%$ of newly diagnosed pancreatic cancer patients are already in a cachectic state (2). Importantly, cancer cachexia is associated with chemotherapy-related toxicity, decreased physical performance status, and a reduced quality of life, and drastically worsens the 5-year survival rate of patients with pancreatic cancer $(1,3)$.

Skeletal muscle mass depletion or 'sarcopenia' is a key feature of cancer cachexia, and contributes to progressive weight loss and a decline in functional abilities. As a result, skeletal muscle is the focus of research in the cachexia field (4-7). However, cardiac muscle has recently also been implicated in the cachexia disease process, with pronounced atrophy and dysfunction in cachectic individuals with pancreatic, lung, or colorectal cancer $(8,9)$. Smooth muscle aberrations in cachexia have not been reported so far, despite the abundance of smooth muscle and its importance for normal function of all hollow organs including the blood vessels, the lungs, the urogenital system, and the gastro-intestinal tract. In support of a role for smooth muscle in the pathophysiology of cachexia, a recent study by Zhou et al. identified symptoms of early satiety, diarrhoea, and constipation in cachectic cancer patients that could be secondary to disturbed gastrointestinal (GI) motility, a process intricately linked to proper intestinal smooth muscle function (10). Moreover, several studies have described Gl-motility disorders such as gastroparesis in patients with malignancies that are frequently accompanied by cachexia (11-15).

The musculature of the gastrointestinal wall consists of an inner circular and an outer longitudinal smooth muscle layer, which are both important for the contractile functionality of the Gl-tract (16). Whereas both skeletal and smooth muscle generate contractile forces by actin-myosin crossbridge interactions between sliding filaments, the contractile apparatus of smooth muscle cells (SMCs) consists of SMC-specific actin and myosin isoforms complemented with SMC-specific contractile proteins such as smoothelin $(17,18)$. Smoothelin is specifically expressed in fully differentiated (contractile) smooth muscle cells. In vitro studies have revealed that smoothelin can bind physically to $\alpha$-smooth muscle actin, pointing toward a direct role of smoothelin in contraction (19, 20). Two adult isoforms of smoothelin have been reported that are expressed in a tissue specific manner: a $59 \mathrm{kDa}$ isoform, smoothelin-A, in visceral and urogenital tissues, and a $110 \mathrm{kDa}$ isoform, smoothelin-B, in vascular tissues $(21,22)$. In support of a direct role of smoothelin in the contractile functionality of the GI-tract, Niessen et al showed that a deficiency of smoothelin-A resulted in impaired contraction of intestinal SMCs and increased intestinal muscle mass (23). These defects caused obstruction, starvation, and ultimately, premature death in smoothelin-A/B knockout mice. Loss of smoothelin expression in the intestinal smooth musculature could therefore have important implications for cachectic cancer patients. 
In response to injury and disease, SMCs display remarkable plasticity and are capable to undergo profound and reversible changes in phenotype, a process referred to as 'phenotypic switching' (24). This phenomenon has been most thoroughly studied in vascular smooth muscle. In response to injury to the vessel wall, the expression of SMC-specific contractile proteins like smooth muscle myosin heavy chain, smoothelin, SM22 $\alpha$, and smcalponin is suppressed, whereas proteins required for synthetic, migratory, and proliferative functions are upregulated (18). To date, little is known about the molecular mechanisms that result in the phenotypic remodelling of intestinal smooth muscle in $\mathrm{GI}$ diseases. Studies on the intestine of patients with Crohn's disease, a chronic inflammatory bowel disease, have revealed profound thickening of the intestinal smooth musculature. In line, in vitro exposure of intestinal SMCs to inflammatory cytokines triggers phenotypic modulation, as evident from an impaired contractile activity, cytoskeletal alterations, decreased expression of smooth muscle differentiation markers, and secretion of proinflammatory cytokines (25-29).

Next to smooth muscle contractile activity, the generation of the contractile forces by the gastrointestinal musculature is further regulated by the enteric nervous system (ENS), consisting of the myenteric plexuses and the interstitial cells of Cajal (ICC). Myenteric plexuses are located between the longitudinal and circular smooth muscle layers and directly regulate relaxation and contraction of the intestinal wall. Structural alterations in the myenteric plexus have previously been observed in tumour-bearing rats, and has been shown to result in a decreased upper GI transit (30). ICC are in close association with both myenteric plexuses and SMCs and generate spontaneous pacemaker currents that drive SMC contractile activities (31).

Given the frequent $\mathrm{Gl}$ symptoms reported in patients with cancer cachexia and the important role of smooth muscle, the myenteric plexuses, and ICC in coordinating intestinal contractile activity, we hypothesized that cachectic cancer patients could display alterations in intestinal SMC phenotype, the myenteric plexus, and/or ICC abundance. We investigated these intestinal musculature characteristics in a cohort of pancreatic cancer patients with and without sarcopenia. 


\section{Materials and Methods}

\section{Patient cohort}

Archived formalin-fixed paraffin-embedded (FFPE) jejunum tissue sections from patients who underwent a Whipple procedure between 2008 and 2013 were obtained from the Pathology Department at the Maastricht University Medical Center (MUMC), the Netherlands. Jejunum tissue sections were included for morphometric and immunohistochemical analysis if the patients had pathology- or radiology-proven pancreatic ductal adenocarcinoma. This study has been approved by the local Medical Ethics Committee and has been performed in accordance with the ethical standards laid down in the 1964 Declaration of Helsinki. The Medical Ethical Committee waived the requirement to obtain informed consent.

\section{Computed tomography scan analysis}

Pre-operative computed tomography (CT) scans were analysed as previously described (32). In short, a single slice at the level of the third lumbar vertebra (L3) of each patient was selected. CT scans were analysed using sliceOmatic 5.0 (TomoVision, Magog, Canada) software for Microsoft Windows ${ }^{\circledR}$. The total cross-sectional area $\left(\mathrm{cm}^{2}\right)$ of skeletal muscle was determined by using predefined $\mathrm{HU}$ ranges $(-29$ to $150 \mathrm{HU})$. The total area of skeletal muscle was normalized for stature to compute the L3-skeletal muscle index (L3-SMI) in $\mathrm{cm}^{2} / \mathrm{m}^{2}$.

The L3-SMI provides a good estimate of total body skeletal muscle mass. Previously published sex-specific cut-off values for L3-SMI (L3-SMI $<41 \mathrm{~cm}^{2} / \mathrm{m}^{2}$ for women, L3-SMI $<43$ $\mathrm{cm}^{2} / \mathrm{m}^{2}$ for men with BMI $<25 \mathrm{~kg} / \mathrm{m}^{2}$ and L3-SMI $<53 \mathrm{~cm}^{2} / \mathrm{m}^{2}$ for men with BMI $\geq 25 \mathrm{~kg} / \mathrm{m}^{2}$ ) were used to assign patients of the current cohort to either a sarcopenia or non-sarcopenia group (33).

\section{Immunohistochemical stainings}

Paraffin-embedded jejunum tissue sections $(4 \mu \mathrm{m})$ were deparaffinized through xylene and rehydrated through graded ethanol to water. Endogenous peroxidase activity was blocked by incubation with $0.6 \%(\mathrm{v} / \mathrm{v}) \mathrm{H}_{2} \mathrm{O}_{2}$ for $15 \mathrm{~min}$. The slides were rinsed three times with $1 \mathrm{x}$ Tris-buffered saline (TBS) and incubated for $30 \mathrm{~min}$ in 5\% BSA in 1xTBS/0.1\% (v/v) Triton X100 to reduce background staining. Tissue sections were immunohistochemically stained with primary antibodies against human $\alpha$-smooth muscle actin ( $\alpha$-SMA, 1:200 dilution) (Clone 1A4, Dako \#M0851), smoothelin (Smtn, 1:100 dilution) (Clone R4A) (34), and CD117/c-kit (1:400 dilution) (Dako \#A4502). Subsequently, the slides were washed and incubated with horseradish peroxidase (HRP)-conjugated secondary antibodies (Vector Laboratories, CA, USA). Peroxidase substrate solution (3,3'-Diaminobenzidine, DAB) (Dako) was used to visualize the presence of peroxidase enzyme. Sections were counterstained for $30 \mathrm{sec}$ with Hematoxylin (Merck, Darmstadt), washed, and then mounted with Entellan (Merck, Darmstadt). 


\section{Picro Sirius Red staining}

Paraffin-embedded jejunal tissue sections were deparaffinized through xylene and rehydrated through a graded alcohol series. Tissue sections were stained for $1 \mathrm{~h}$ with $0.1 \%$ (w/v) Picro Sirius Red (Direct Red 80, Sigma) in a saturated aqueous solution of picric acid followed by washing with acidified water $(0.01 \mathrm{~N} \mathrm{HCl})$. The stained tissue sections were dehydrated and mounted by Entellan.

\section{Digitalization images}

Stained jejunal tissue slides were digitalized using the Ventana iScan HT (Version 1.1, Roche, Ventana Medical Systems, Inc.) using a 200x magnification. The scans were opened in Pannoramic Viewer software (Version 1.15.4, 3DHISTECH, Ltd) for additional analysis. Areas of interest (AOI) in the circular and longitudinal smooth muscle layer were manually selected in Pannoramic viewer. AOI were only selected in the cross-sectional orientation of the longitudinal layer (LL) and the transversal section of the circular layer (CL). Stained tissue sections were excluded from analysis when the smooth muscle layers did not meet the orientation criteria or when the tissue morphology was drastically disrupted. Depending on the size of the tissue section, at least 5 representative AOIs were selected for both the inner and outer smooth muscle layer.

\section{Morphometric analyses}

Thickness of the smooth muscle layers was measured by using the distance measurement annotation option in the Pannoramic Viewer software. The thickness was measured between the intermuscular plane displaying the myenteric plexus and the respective borders of the circular and the longitudinal muscle layer. The thickness was measured over the full length of the smooth muscle layer when the longitudinal layer was cross-sectionally orientated and the circular layer transversally orientated.

\section{Histological analysis jejunal FFPE tissue}

DAB staining intensities of $\alpha$-SMA and smoothelin were quantified using ImageJ (Version 1.51h). Each selected $A O I$ of a jejunal tissue section was separated into a DAB and hematoxylin stained image by using the colour deconvolution plugin (H_DAB) in the ImageJ software (35). The pixel intensity was measured throughout both smooth muscle layers. The reciprocal intensity was calculated as described previously and is expressed as arbitrary units (a.u.) (36).

The CD117 (c-Kit) immunohistochemical staining was assessed semi quantitatively based on a previously reported grading system (37). The density of c-Kit immunoreactivity was graded as absent (loss of ICC network), moderate (disturbed ICC network), or abundant (homogeneous ICC network). A similar validated grading system was used to evaluate the degree of collagen deposition (38). In short, the degree of collagen deposition was graded as none when the myenteric plexus was not surrounded by a network of collagen fibres; mild when the myenteric plexus was surrounded by a thin network of collagen fibres, and severe when the myenteric plexus was surrounded by a dense network of collagen fibres. 
Statistical analysis

Data are expressed as the mean \pm standard deviation. Patient data and outcome parameters were entered in IBM SPSS 24 for Microsoft Windows ${ }^{\circledR}$ and statistical analyses were performed using the Mann-Whitney $\mathrm{U}$ test (continuous variables) to compare differences between two groups. The Fisher's exact test and Pearson's chi-square $\left(x^{2}\right)$ test were used for between-group comparisons of categorical variables. A $p$-value of $p<0.05$ was considered statistically significant. 


\section{Results}

\section{Patient characteristics}

Relevant characteristics of the patients with and without preoperative sarcopenia are shown in table 1. A total of 57 patients were assigned to the sarcopenia $(n=26)$ or nonsarcopenia ( $n=31$ ) group based on established sex-specific cut-off values (33). Mean age and BMI were not significantly different between the two groups. The L3-SMI of the sarcopenia group was $44.7 \pm 5.1 \mathrm{~cm}^{2} / \mathrm{m}^{2}$ for males and $35.4 \pm 3.4 \mathrm{~cm}^{2} / \mathrm{m}^{2}$ for females versus $50.9 \pm 6.5$ $\mathrm{cm}^{2} / \mathrm{m}^{2}$ for males and $46.1 \pm 4.8 \mathrm{~cm}^{2} / \mathrm{m}^{2}$ for females in the non-sarcopenia group $(p<0.001)$.

Table 1: General patient characteristics

\begin{tabular}{lcccc}
\hline & $\begin{array}{c}\text { Total } \\
(\mathrm{n}=57)\end{array}$ & $\begin{array}{c}\text { Non-Sarcopenia } \\
(\mathrm{n}=26)\end{array}$ & $\begin{array}{c}\text { Sarcopenia } \\
(\mathrm{n}=31)\end{array}$ & $p$-value \\
\hline Gender & $25(44 \%)$ & $13(50 \%)$ & $12(39 \%)$ & $\mathrm{p}=0.43$ \\
$\quad$ Male & $32(56 \%)$ & $13(50 \%)$ & $19(61 \%)$ & \\
$\quad$ Female & $66.02( \pm 9.2)$ & $65.0( \pm 8.4)$ & $66.8( \pm 10.0)$ & $\mathrm{p}=0.34$ \\
Age (years) & $24.1( \pm 3.7)$ & $24.1( \pm 3.9)$ & $24.1( \pm 3.7)$ & $\mathrm{p}=0.96$ \\
Body mass index $\left(\mathrm{kg} / \mathrm{m}^{2}\right)$ & & & & \\
& & & & \\
L3-Skeletal muscle index & & & & \\
(cm $\left./ \mathrm{m}^{2}\right)$ & & & & \\
$\quad$ Male & $39.9( \pm 6.5)$ & $50.9( \pm 6.5)$ & & \\
Female & & $46.1( \pm 4.8)$ & $35.4( \pm 3.4)$ & $\mathrm{p}<0.001$ \\
& & & & \\
TNM Classification $(N=45)$ & $14(31.1 \%)$ & $8(38.1 \%)$ & $6(25.0 \%)$ & $\mathrm{p}=0.52$ \\
$\quad$ Stage IIA & $31(68.9 \%)$ & $13(61.9 \%)$ & $18(75.0 \%)$ & \\
$\quad$ Stage IIB & & & & \\
\hline
\end{tabular}

${ }^{*}$ Patients were assigned to a sarcopenia or non-sarcopenia group based on sex-specific cut-off values for L3-SMI (L3-SMI $<41 \mathrm{~cm}^{2} / \mathrm{m}^{2}$ for women, L3-SMI $<43 \mathrm{~cm}^{2} / \mathrm{m}^{2}$ for men with BMI $<25 \mathrm{~kg} / \mathrm{m}^{2}$ and L3$\mathrm{SMI}<53 \mathrm{~cm}^{2} / \mathrm{m}^{2}$ for men with $\mathrm{BMI} \geq 25 \mathrm{~kg} / \mathrm{m}^{2}$ )

Similar intestinal smooth muscle wall thickness in sarcopenic and non-sarcopenic patients

To investigate whether intestinal smooth muscle wall thickness differed between pancreatic cancer patients with and without sarcopenia, we first performed morphometric analyses. Representative images of the intestinal smooth muscle wall in non-sarcopenic and sarcopenic patients are presented in Fig. 1A. Total intestinal smooth muscle wall thickness in patients with sarcopenia was not significantly different from that of the non-sarcopenia group (1661 $\pm 661 \mu \mathrm{m}$ vs. $1439 \pm 449 \mu \mathrm{m}$, respectively $\mathrm{p}=0.41$ ) (Fig. 1B). Analysis of the individual smooth muscle layers also did not reveal differences between the sarcopenia and non-sarcopenia group (circular muscle layers (CL): $1090 \pm 455 \mu \mathrm{m}$ vs. $917 \pm 303 \mu \mathrm{m}, \mathrm{p}=0.23$ and longitudinal layers (LL): (571 $\pm 273 \mu \mathrm{m}$ vs $523 \pm 198 \mu \mathrm{m}, \mathrm{p}=0.69)$ (Fig. 1C). 

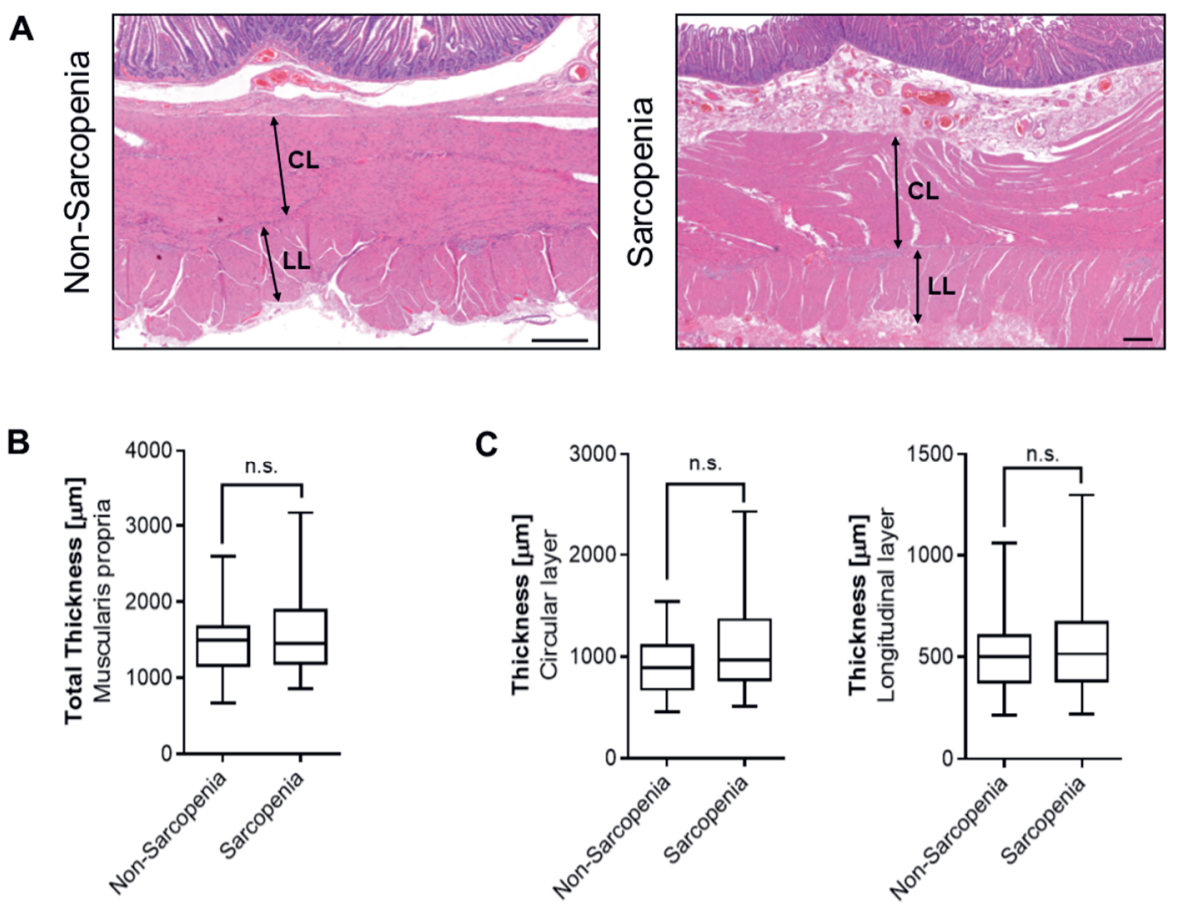

Figure 1: Thickness of the intestinal smooth musculature of pancreatic cancer patients. (A) Representative hematoxylin and eosin stainings of the intestinal smooth muscle wall of nonsarcopenic (left) and sarcopenic (right) pancreatic cancer patients. Scale bar $=500 \mu \mathrm{m}$. (B) Total thickness of the muscularis propria and (C) the thicknesses of the longitudinal (LL) and circular (CL) muscle layer were measured in $\mu \mathrm{m}$. Results are presented in box plot graphs. The boxes represent the interquartile range (IQR) in which the medians are indicated as a bar. Differences between the nonsarcopenia $(n=26)$ and sarcopenia $(n=31)$ group were analyzed using the non-parametric MannWhitney $U$ test.

Reduced smoothelin expression in the intestinal muscle wall of sarcopenic patients To assess a potential association between skeletal muscle sarcopenia and the intestinal smooth muscle contractile phenotype, we next investigated functionally relevant proteins of the smooth muscle contractile apparatus in the intestinal musculature in the two patient groups. Abundant expression of $\alpha$-SMA, the major actin isoform in smooth muscle, was observed in both groups, with consistently higher staining intensities in the longitudinal layer compared to the circular layer. No significant differences in the staining intensity of $\alpha$ SMA were detected between the sarcopenia group and the non-sarcopenia group (CL: $91.5 \pm 22.3$ a.u. vs. $95.8 \pm 18.8$ a.u., $p=0.46$; LL: $130.2 \pm 19.0$ a.u. vs. $129.7 \pm 17.8$ a.u., $p=0.89$ ) (Fig. 2A, C).

Whereas $\alpha$-SMA is expressed in both contractile and synthetic SMCs and has been shown to be relatively unresponsive to microenvironmental stimuli that affect the SMC phenotype (e.g. tumour-derived mediators, inflammatory cytokines), smoothelin is a more sensitive marker specifically expressed in contractile SMCs. Whereas we did not observe 
differences in the staining intensity of smoothelin in the circular muscle layer of both groups (118.5 \pm 24.7 a.u. vs. $116.6 \pm 21.0$ a.u., $p=0.67$ ), staining intensities in the longitudinal muscle layer of pancreatic cancer patients with sarcopenia were significantly reduced $(143.0 \pm 22.6$ a.u. vs. $125.4 \pm 29.3$ a.u., $p=0.02$ ) (Fig 2B, D).

A
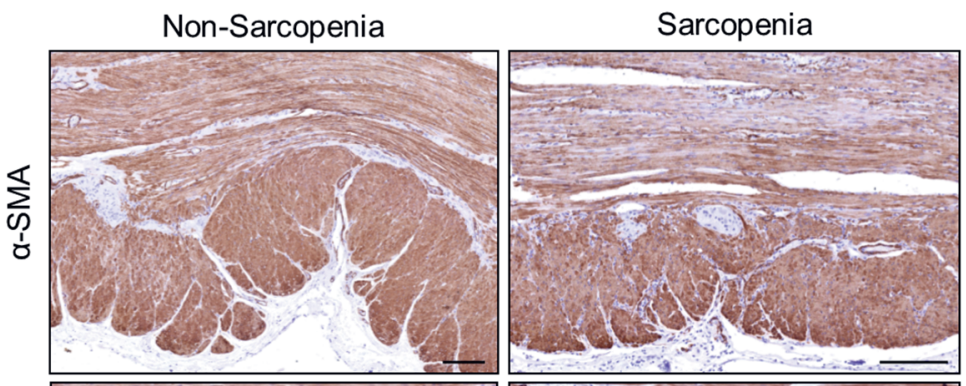

B
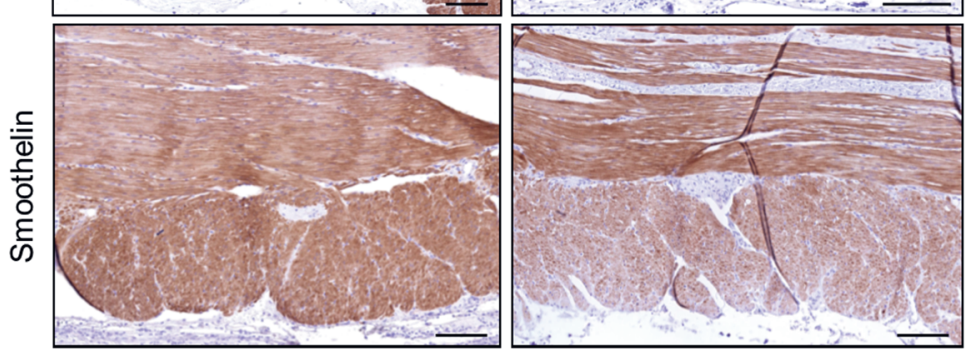

C

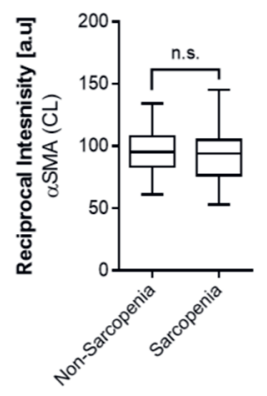

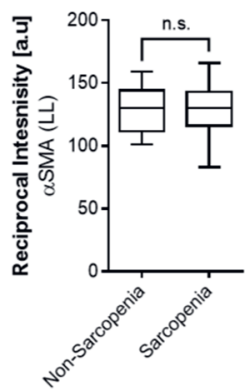

D
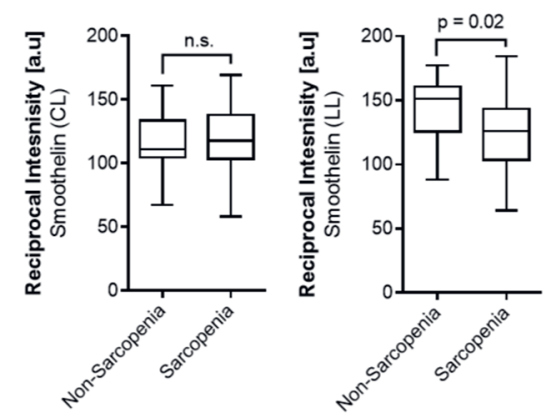

Figure 2: Expression of contractile smooth muscle-specific markers in the intestinal smooth muscle layer of pancreatic cancer patients. (A) Representative immuno-histochemical staining of $\alpha$-smooth muscle actin ( $\alpha$-SMA) and (B) Smoothelin in the intestinal smooth muscle wall of non-sarcopenic (left) and sarcopenic (right) pancreatic cancer patients. Scale bar $=200 \mu \mathrm{m}$. (C) The reciprocal staining intensities of $\alpha$-SMA and (D) Smoothelin in the circular (CL) and longitudinal (LL) smooth muscle layers are presented in box plot graphs. The boxes represent the interquartile range (IQR) in which the medians are indicated as a bar. Differences between the non-sarcopenia $(n=26)$ and sarcopenia $(n=31)$ group were analyzed using the non-parametric Mann-Whitney $U$ test, ${ }^{*} p<0.05$. 
Excessive collagen deposition around the myenteric plexuses in patients with sarcopenia

Since the reduced smoothelin expression in patients with sarcopenia suggested that their intestinal SMCs had adopted a synthetic phenotype, we next investigated whether increased ECM deposition was present in the intestinal musculature of pancreatic cancer patients with sarcopenia. In line with a more synthetic SMC phenotype, visualization of collagen fibres by Picro Sirius Red staining revealed pronounced fibrosis in $40 \%$ of the sarcopenic patients, specifically around the myenteric plexuses and between the inner and outer muscle layers (Fig. 3A). Semi-quantitative scoring showed that the frequency of severe collagen deposition around the myenteric plexus was more than twice as high in the sarcopenia group (40\%) than in the non-sarcopenia group (19\%, p=0.04) (Fig. 3B).

A
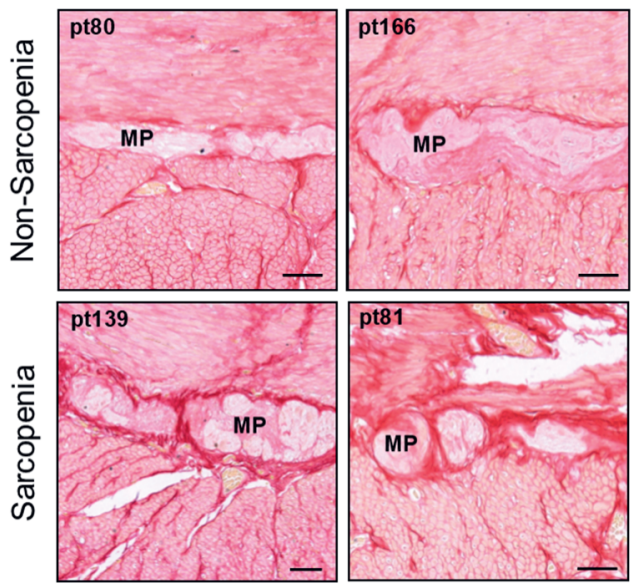
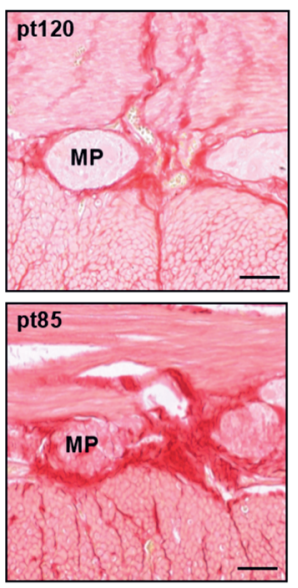

B

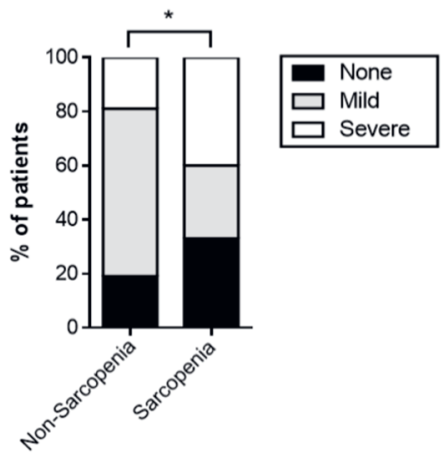

Figure 3: Increased collagen deposition around the myenteric plexuses in the intestinal wall of sarcopenic pancreatic cancer patients. (A) Collagen deposition in the intestinal smooth muscle wall was visualized by Picro Sirius Red staining. Representative images of the myenteric plexus (indicated as MP) in the smooth musculature of the non-sarcopenia (upper panel) and sarcopenia (lower panel) group are shown. Scale bar $=200 \mu \mathrm{m}$. (B) Severe collagen deposition around the myenteric plexus was more often observed in patients with sarcopenia as compared with non-sarcopenia patients $(* \mathrm{p}=0.04)$. 
Distribution of the ICC network within the intestinal smooth musculature

The interstitial cells of Cajal that act as pacemakers for intestinal smooth muscle contraction are characterized by expression of the tyrosine kinase receptor CD117/c-kit. Immunohistochemical staining of the intestinal musculature with an anti-CD117 antibody revealed a normal, homogeneous distribution of the ICC network in $42 \%$ of the patients in the non-sarcopenia group compared to $26 \%$ of the patients in the sarcopenia group (Fig. $4 \mathrm{~A}, \mathrm{~B})$. Complete loss of ICC was more often observed in pancreatic cancer patients with sarcopenia ( $16 \%$ vs. $4 \%)$, but the observed differences between the groups were not statistically significant (Fig. 4B) $(\mathrm{p}=0.20)$.

\section{A}
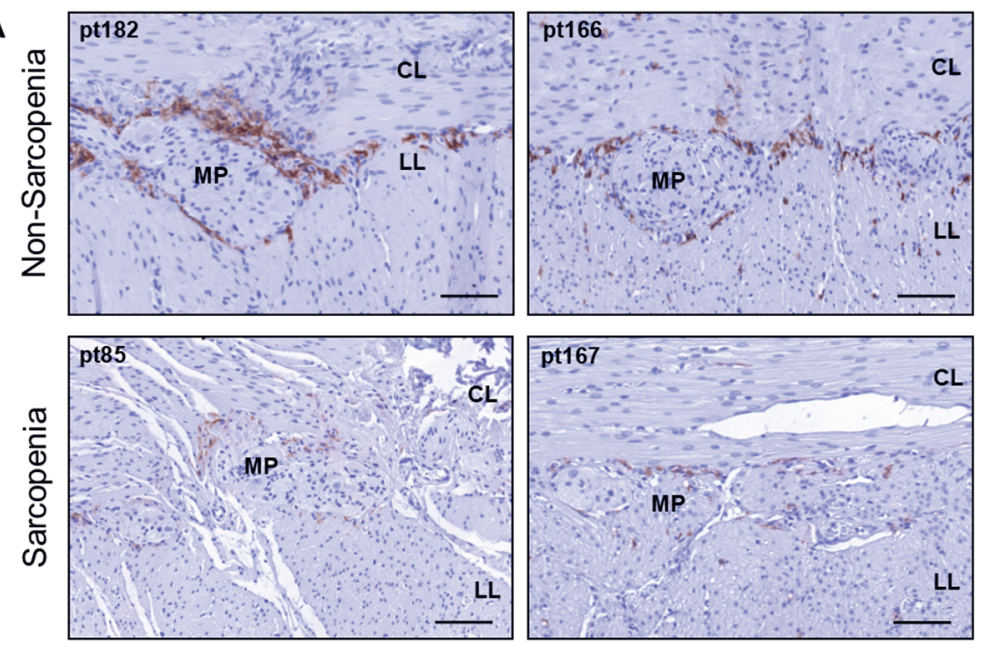

B

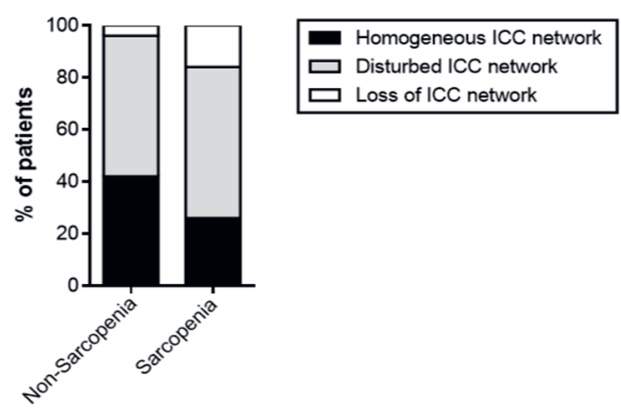

Figure 4: Distribution of the Interstitial Cells of Cajal in the intestinal musculature. (A) Representative images of CD117/c-kit stained Interstitial Cells of Cajal (ICC) in the smooth musculature of nonsarcopenic (upper panel) and sarcopenic (lower panel) pancreatic cancer patients. CD117/c-kit positive ICC cells stained brown and are located between the circular (CL) and longitudinal (LL) smooth muscle layers. $\mathrm{MP}=$ myenteric plexus. Scale bar $=100 \mu \mathrm{m}$. (B) No differences were observed between the distribution of the ICC network in the smooth musculature of non-sarcopenic and sarcopenic patients $(p=0.20)$. 


\section{Discussion}

In the current study, we investigated whether sarcopenia in patients with pancreatic cancer was associated with aberrations in their intestinal smooth musculature. We observed reduced expression of the contractile SMC-specific protein smoothelin, which reflects deficiencies of smooth muscle contractile function and is indicative of a synthetic SMC phenotype. The pronounced collagen deposition around the myenteric plexus in sarcopenic patients corroborates this notion and suggests that regulation of smooth muscle contractility may also be compromised in sarcopenic patients.

Muscle wasting in cancer cachexia is considered to be induced by tumour-derived factors that directly or indirectly activate intracellular signaling pathways disturbing cell homeostasis. It is likely that these systemic circulating factors also affect the intestinal smooth musculature of cachectic patients. In contrast to skeletal muscle, which becomes atrophic under stress conditions, SMCs show remarkable resilience and phenotypic plasticity in response to stress stimuli such as inflammation $(28,39)$, hormonal changes $(40)$, chronic stretch or pressure (41), and obstruction (42). Under these conditions, contractile SMCs revert to a synthetic phenotype characterized by increased extracellular matrix production and/or higher proliferation rates, frequently resulting in thickening of the smooth muscle tissue, decreased compliance, and reduction in relaxation as well as contraction potential (43-45). Whereas overall thickness of the intestinal smooth muscle layers was similar between sarcopenic and non-sarcopenic patients, collagen deposition was significantly increased in patients with sarcopenia. In line with this observation, tissue fibrosis as a consequence of increased ECM deposition has previously been shown in cancer cachexia. For example, enhanced ECM deposition surrounding cardiomyocytes has been observed in a mouse model of cancer-induced cachexia $(9,46)$, and profound collagen accumulation was demonstrated in subcutaneous adipose tissue of cachectic cancer patients $(47,48)$. These studies may indicate that tumour-derived factors induce generalized tissue fibrosis which, in smooth muscle, could be attributable to phenotypic switching of SMCs from a contractile to a synthetic state.

In line with this, expression of the contractile phenotype-restricted smooth muscle marker smoothelin was reduced in sarcopenic patients. Although smoothelin is a widely used smooth muscle phenotype marker, only few studies have evaluated its function and distribution in $\mathrm{GI}$ motility disorders $(23,49,50)$. Niessen and colleagues reported that loss of smoothelin in a knock-out mouse model resulted in irregular slow wave patterns, impaired intestinal contraction, and hampered intestinal transit (23). These data emphasize the important functional role of smoothelin in GI-motility and support the concept that reduced smoothelin levels in sarcopenic patients are indicative of deficiencies of the SMC contractile apparatus potentially contributing to Gl-dysmotility. Moreover, hampered intestinal transit in smoothelin-A/B knockout mice causes obstruction, starvation, and ultimately premature death, indicating that minimal differences in smoothelin expression 
may have direct functional implications for GI motility in humans. In line with our findings of reduced smoothelin immunoreactivity specifically in the longitudinal muscle layer, Chan and colleagues showed that smoothelin was selectively reduced in the longitudinal layer in patients with colonic inertia (49). This may have particularly important implications for GI motility given the special functions of the longitudinal muscle layer in peristalsis of the intestine $(51,52)$.

Although primary defects in the smooth musculature can greatly impact GI motility, generation of functional contractile forces by SMCs also relies on the interplay between SMCs and ICC. In the present study, we observed notable differences in the distribution of ICC between patients ranging from well-organized ICC networks to the complete loss of ICC. ICC are responsible for the generation of electrical slow wave activity that is propagated through networks of ICC to electrically coupled SMCs, resulting in SMC contractile activities (53). Previous research has highlighted the importance of ICC in the regulation of GI motility by showing that loss of ICC or disruption of the ICC network is associated with GI motility disorders $(16,54-56)$. Additionally, genetic loss-of-function studies by Klein et al. showed that ICC are essential for the transmission of signals from enteric neurons to gastrointestinal SMCs and are therefore required to orchestrate peristaltic motor activity of the gut (57). Although we did not observe significant differences in the distribution of ICC between sarcopenic and non-sarcopenic patients, we cannot exclude that the transmission of electrical signals between ICC and SMCs may be inhibited by the presence of excessive collagen deposition.

Given the importance of smooth muscle contractility for GI function and motility, the intestinal SMC disturbances that we observed in sarcopenic patients could have significant implications for their nutrient intake, transport, absorption, and faecal output and thus contribute to the development and progression of cachexia. In line with this, previous studies have reported that digestive and motility disorders (e.g. anorexia, dysphagia, constipation, diarrhoea, and abdominal pain) are associated with cancer $(10,58)$. However, so far, smooth muscle dysfunction has not been studied as a possible cause underlying these effects. Interestingly, intestinal inflammation and immune activation are often accompanied by GI-dysmotility (59), and pro-inflammatory cytokines induce collagen and metalloproteinases production, which contribute to intestinal fibrosis $(28,39)$. Thus, the systemic inflammation that is considered to be the major driver of cancer cachexia could be involved in the smooth muscle aberrations reported here. Altogether, this highlights the potential importance of smooth muscle in the pathogenesis of cancer cachexia and warrants further investigations into the role of this tissue.

There are some limitations to this study. We analysed archived-jejunal tissue sections that were not sampled and processed for analysis of intestinal smooth muscle tissue. To minimize the impact of this, we only selected tissue sections in which the circular and longitudinal muscle layers were similarly orientated. Furthermore, the retrospective design of this study resulted in missing data of some patients (e.g. weight loss, C-reactive protein). 
These data would have been helpful to better define the cachectic status of patients (60). In addition, data regarding the functionality of the GI-tract are lacking.

In conclusion, our data show that muscle aberrations in cancer cachexia are not limited to skeletal and cardiac tissue, but also apparent in intestinal smooth muscle. The reduced contractile smooth muscle marker expression and collagen accumulation around the myenteric plexus of sarcopenic patients indicate that both smooth muscle contractile function and its regulation could be compromised in cancer patients with sarcopenia, potentially explaining the frequent GI problems reported in this patient population. 


\section{References}

1. Fearon K, Strasser F, Anker SD, Bosaeus I, Bruera E, Fainsinger RL, et al. Definition and classification of cancer cachexia: an international consensus. Lancet Oncol. 2011;12(5):48995.

2. Talbert EE, Lewis HL, Farren MR, Ramsey ML, Chakedis JM, Rajasekera P, et al. Circulating monocyte chemoattractant protein-1 (MCP-1) is associated with cachexia in treatmentnaive pancreatic cancer patients. J Cachexia Sarcopenia Muscle. 2018;9(2):358-68.

3. Bachmann J, Heiligensetzer M, Krakowski-Roosen H, Buchler MW, Friess H, Martignoni ME. Cachexia worsens prognosis in patients with resectable pancreatic cancer. J Gastrointest Surg. 2008;12(7):1193-201.

4. Argiles JM, Busquets S, Stemmler B, Lopez-Soriano FJ. Cancer cachexia: understanding the molecular basis. Nat Rev Cancer. 2014;14(11):754-62.

5. Fearon KC, Glass DJ, Guttridge DC. Cancer cachexia: mediators, signaling, and metabolic pathways. Cell Metab. 2012;16(2):153-66.

6. Penna F, Ballaro R, Beltra M, De Lucia S, Costelli P. Modulating Metabolism to Improve Cancer-Induced Muscle Wasting. Oxid Med Cell Longev. 2018;2018:7153610.

7. $\quad$ Sandri M. Protein breakdown in cancer cachexia. Semin Cell Dev Biol. 2016;54:11-9.

8. Barkhudaryan A, Scherbakov N, Springer J, Doehner W. Cardiac muscle wasting in individuals with cancer cachexia. ESC Heart Fail. 2017;4(4):458-67.

9. Springer J, Tschirner A, Haghikia A, von Haehling S, Lal H, Grzesiak A, et al. Prevention of liver cancer cachexia-induced cardiac wasting and heart failure. Eur Heart J. 2014;35(14):932-41.

10. Zhou T, Yang K, Thapa S, Liu H, Wang B, Yu S. Differences in Symptom Burden Among Cancer Patients With Different Stages of Cachexia. J Pain Symptom Manage. 2017;53(5):919-26.

11. Barkin JS, Goldberg RI, Sfakianakis GN, Levi J. Pancreatic carcinoma is associated with delayed gastric emptying. Dig Dis Sci. 1986;31(3):265-7.

12. Burger JA, Liberov B, Yurd F, Loffeld RJ. A Case of Extreme Gastroparesis due to an Occult Small Cell Cancer of the Lung. Case Rep Gastrointest Med. 2013;2013:182962.

13. Caras S, Laurie S, Cronk W, Tompkins W, Brashear R, McCallum RW. Case report: pancreatic cancer presenting with paraneoplastic gastroparesis. Am J Med Sci. 1996;312(1):34-6.

14. Hejazi RA, Zhang D, McCallum RW. Gastroparesis, pseudoachalasia and impaired intestinal motility as paraneoplastic manifestations of small cell lung cancer. Am J Med Sci. 2009;338(1):69-71.

15. Kelly D, Moran C, Maher M, O'Mahony S. Malignancy-associated gastroparesis: an important and overlooked cause of chronic nausea and vomiting. BMJ Case Rep. 2014;2014.

16. Sanders KM, Koh SD, Ro S, Ward SM. Regulation of gastrointestinal motility--insights from smooth muscle biology. Nat Rev Gastroenterol Hepatol. 2012;9(11):633-45.

17. Gordon AM, Homsher E, Regnier M. Regulation of contraction in striated muscle. Physiol Rev. 2000;80(2):853-924.

18. Owens GK, Kumar MS, Wamhoff BR. Molecular regulation of vascular smooth muscle cell differentiation in development and disease. Physiol Rev. 2004;84(3):767-801.

19. Niessen P, Clement S, Fontao L, Chaponnier C, Teunissen B, Rensen S, et al. Biochemical evidence for interaction between smoothelin and filamentous actin. Exp Cell Res. 2004;292(1):170-8.

20. van der Loop FT, Schaart G, Timmer ED, Ramaekers FC, van Eys GJ. Smoothelin, a novel cytoskeletal protein specific for smooth muscle cells. J Cell Biol. 1996;134(2):401-11.

21. van Eys GJ, Voller MC, Timmer ED, Wehrens XH, Small JV, Schalken JA, et al. Smoothelin expression characteristics: development of a smooth muscle cell in vitro system and identification of a vascular variant. Cell Struct Funct. 1997;22(1):65-72. 
22. Wehrens XH, Mies B, Gimona M, Ramaekers FC, Van Eys GJ, Small JV. Localization of smoothelin in avian smooth muscle and identification of a vascular-specific isoform. FEBS Lett. 1997;405(3):315-20.

23. Niessen P, Rensen S, van Deursen J, De Man J, De Laet A, Vanderwinden JM, et al. Smoothelin-a is essential for functional intestinal smooth muscle contractility in mice. Gastroenterology. 2005;129(5):1592-601.

24. Scirocco A, Matarrese P, Carabotti M, Ascione B, Malorni W, Severi C. Cellular and Molecular Mechanisms of Phenotypic Switch in Gastrointestinal Smooth Muscle. J Cell Physiol. 2016;231(2):295-302.

25. Blennerhassett MG, Vignjevic $P$, Vermillion DL, Collins SM. Inflammation causes hyperplasia and hypertrophy in smooth muscle of rat small intestine. Am J Physiol. 1992;262(6 Pt 1):G1041-6.

26. Li C, Kuemmerle JF. Mechanisms that mediate the development of fibrosis in patients with Crohn's disease. Inflamm Bowel Dis. 2014;20(7):1250-8.

27. Matarrese P, Petitta C, Scirocco A, Ascione B, Ammoscato F, Di Natale G, et al. Antioxidants counteract lipopolysaccharide-triggered alterations of human colonic smooth muscle cells. Free Radic Biol Med. 2012;53(11):2102-11.

28. Nair DG, Miller KG, Lourenssen SR, Blennerhassett MG. Inflammatory cytokines promote growth of intestinal smooth muscle cells by induced expression of PDGF-Rbeta. J Cell Mol Med. 2014;18(3):444-54.

29. Owens MW, Grisham MB. Cytokines increase proliferation of human intestinal smooth muscle cells: possible role in inflammation-induced stricture formation. Inflammation. 1993;17(4):481-7.

30. Marega P, Liberti EA, Freitas JJS, Kietzer KS. Walker-256 tumor alters morphology of intestinal myenteric plexus in rats. Neurogastroenterol Motil. 2018.

31. Takaki M. Gut pacemaker cells: the interstitial cells of Cajal (ICC). J Smooth Muscle Res. 2003;39(5):137-61.

32. van Dijk DP, Bakens MJ, Coolsen MM, Rensen SS, van Dam RM, Bours MJ, et al. Low skeletal muscle radiation attenuation and visceral adiposity are associated with overall survival and surgical site infections in patients with pancreatic cancer. J Cachexia Sarcopenia Muscle. 2017;8(2):317-26.

33. Martin L, Birdsell L, Macdonald N, Reiman T, Clandinin MT, McCargar L, et al. Cancer cachexia in the age of obesity: skeletal muscle depletion is a powerful prognostic factor, independent of body mass index. J Clin Oncol. 2013;31(12):1539-47.

34. van der Loop FT, Gabbiani G, Kohnen G, Ramaekers FC, van Eys GJ. Differentiation of smooth muscle cells in human blood vessels as defined by smoothelin, a novel marker for the contractile phenotype. Arterioscler Thromb Vasc Biol. 1997;17(4):665-71.

35. Ruifrok AC, Johnston DA. Quantification of histochemical staining by color deconvolution. Anal Quant Cytol Histol. 2001;23(4):291-9.

36. Rizzardi AE, Johnson AT, Vogel RI, Pambuccian SE, Henriksen J, Skubitz AP, et al. Quantitative comparison of immunohistochemical staining measured by digital image analysis versus pathologist visual scoring. Diagn Pathol. 2012;7:42.

37. Forster J, Damjanov I, Lin Z, Sarosiek I, Wetzel P, McCallum RW. Absence of the interstitial cells of Cajal in patients with gastroparesis and correlation with clinical findings. J Gastrointest Surg. 2005;9(1):102-8.

38. Park KS, Cho KB, Hwang IS, Park JH, Jang BI, Kim KO, et al. Characterization of smooth muscle, enteric nerve, interstitial cells of Cajal, and fibroblast-like cells in the gastric musculature of patients with diabetes mellitus. World J Gastroenterol. 2016;22(46):10131-9.

39. Shea-Donohue T, Notari L, Sun R, Zhao A. Mechanisms of smooth muscle responses to inflammation. Neurogastroenterol Motil. 2012;24(9):802-11. 
40. Ohguchi H, Mitsui R, Imaeda K, Joh T, Hashitani H. Mechanisms of PTHrP-induced inhibition of smooth muscle contractility in the guinea pig gastric antrum. Neurogastroenterol Motil. 2017;29(12).

41. Shi XZ. Mechanical Regulation of Gene Expression in Gut Smooth Muscle Cells. Front Physiol. 2017;8:1000.

42. MacDonald JA. Smooth muscle phenotypic plasticity in mechanical obstruction of the small intestine. Neurogastroenterol Motil. 2008;20(7):737-40.

43. James AL, Elliot JG, Jones RL, Carroll ML, Mauad T, Bai TR, et al. Airway smooth muscle hypertrophy and hyperplasia in asthma. Am J Respir Crit Care Med. 2012;185(10):1058-64.

44. Tham YK, Bernardo BC, Ooi JY, Weeks KL, McMullen JR. Pathophysiology of cardiac hypertrophy and heart failure: signaling pathways and novel therapeutic targets. Arch Toxicol. 2015;89(9):1401-38.

45. Thorpe A, Neal D. Benign prostatic hyperplasia. Lancet. 2003;361(9366):1359-67.

46. Tian M, Nishijima Y, Asp ML, Stout MB, Reiser PJ, Belury MA. Cardiac alterations in cancerinduced cachexia in mice. Int J Oncol. 2010;37(2):347-53.

47. Alves MJ, Figueredo RG, Azevedo FF, Cavallaro DA, Neto NI, Lima JD, et al. Adipose tissue fibrosis in human cancer cachexia: the role of TGFbeta pathway. BMC Cancer. 2017;17(1):190.

48. Batista ML, Jr., Henriques FS, Neves RX, Olivan MR, Matos-Neto EM, Alcantara PS, et al. Cachexia-associated adipose tissue morphological rearrangement in gastrointestinal cancer patients. J Cachexia Sarcopenia Muscle. 2016;7(1):37-47.

49. Chan OT, Chiles L, Levy M, Zhai J, Yerian LM, Xu H, et al. Smoothelin expression in the gastrointestinal tract: implication in colonic inertia. Appl Immunohistochem Mol Morphol. 2013;21(5):452-9.

50. Wedel T, Van Eys GJ, Waltregny D, Glenisson W, Castronovo V, Vanderwinden JM. Novel smooth muscle markers reveal abnormalities of the intestinal musculature in severe colorectal motility disorders. Neurogastroenterol Motil. 2006;18(7):526-38.

51. Grider JR. Reciprocal activity of longitudinal and circular muscle during intestinal peristaltic reflex. Am J Physiol Gastrointest Liver Physiol. 2003;284(5):G768-75.

52. Smith TK, Robertson WJ. Synchronous movements of the longitudinal and circular muscle during peristalsis in the isolated guinea-pig distal colon. J Physiol. 1998;506 ( Pt 2):563-77.

53. Sanders KM, Kito Y, Hwang SJ, Ward SM. Regulation of Gastrointestinal Smooth Muscle Function by Interstitial Cells. Physiology (Bethesda). 2016;31(5):316-26.

54. Farrugia G. Interstitial cells of Cajal in health and disease. Neurogastroenterol Motil. $2008 ; 20$ Suppl 1:54-63.

55. Sanders KM, Ordog T, Koh SD, Torihashi S, Ward SM. Development and plasticity of interstitial cells of Cajal. Neurogastroenterol Motil. 1999;11(5):311-38.

56. Vanderwinden JM, Rumessen JJ. Interstitial cells of Cajal in human gut and gastrointestinal disease. Microsc Res Tech. 1999;47(5):344-60.

57. Klein S, Seidler B, Kettenberger A, Sibaev A, Rohn M, Feil R, et al. Interstitial cells of Cajal integrate excitatory and inhibitory neurotransmission with intestinal slow-wave activity. Nat Commun. 2013;4:1630.

58. DiBaise JK, Quigley EM. Tumor-related dysmotility: gastrointestinal dysmotility syndromes associated with tumors. Dig Dis Sci. 1998;43(7):1369-401.

59. Akiho H, Ihara E, Motomura Y, Nakamura K. Cytokine-induced alterations of gastrointestinal motility in gastrointestinal disorders. World J Gastrointest Pathophysiol. 2011;2(5):72-81.

60. Argiles JM, Lopez-Soriano FJ, Toledo M, Betancourt A, Serpe R, Busquets S. The cachexia score (CASCO): a new tool for staging cachectic cancer patients. J Cachexia Sarcopenia Muscle. 2011;2(2):87-93. 


\section{CHAPTER 7}

General discussion 


\section{Introduction}

Cancer cachexia is a multifactorial wasting syndrome that is characterized by involuntary loss of body weight and an ongoing loss of skeletal muscle mass (1). Among all cancer types, patients with pancreatic cancer have the highest prevalence of cancer cachexia and often develop the most severe degrees of this syndrome (2). To date, the diagnosis and treatment of cachexia remains a significant clinical challenge. To be able to identify predictive and prognostic biomarkers and to develop effective treatment regimens, there is an urgent need to gain novel insights into the cachexia-inducing mediators and their mechanism of action in human cancer cachexia. Although experimental pre-clinical systems that model human cancer-associated cachexia have tremendously contributed to our current understanding of its pathophysiology, the translation of these findings into clinical benefits remains a major challenge. This thesis described the generation and characterization of pancreatic tumor organoids as a novel model to study cancer-induced cachexia and provided novel insights into human cancer cachexia-induced muscle alterations.

In the following integrative discussion, I will discuss 1 ) the use of tumor organoids as a novel model to study cancer-induced cachexia, 2 ) the potential of using organoids to study tumorhost interactions, 3 ) the identification of cachexia-inducing factors in human cancers, and 4) the assessment of the cachexia status in cancer patients.

\section{Organoids as a novel model to study human cancer-induced cachexia}

In cancer cachexia research, traditional human cancer cell lines are widely used to assess the direct effect of the tumor secretome on target tissues (3-6). However, a major limitation of this approach is that the cachexia-related clinical data of the patients of whom these cancer cells were derived are completely lacking. Also, traditional cell culture models are comparatively artificial since cells are maintained on a stiff two-dimensional (2D) plastic surface in the absence of physiological conditions (i.e. oxygen and nutrient gradients, lack of cellular extracellular matrix interactions). Furthermore, since most of the cell lines used in cachexia research have been cultured in 2D for decades, currently available strains are genetically different compared to the originally isolated tumor cells (7), potentially leading to differential activation and/or inhibition of gene expression programs. This implies that many established pancreatic cell lines will likely have gained or lost cachexia-inducing properties, resulting in inconsistent findings among studies. In addition, given that human cancer cachexia is a heterogeneous clinical syndrome, it is unlikely that the limited number of available established human pancreatic cancer cell lines recapitulate all clinically relevant cachectic properties. 
To surpass these limitations and to be able to link novel mechanistic findings to clinically relevant cachexia-parameters, including the ongoing loss of skeletal muscle mass, animal models have been widely used to model cachexia. Upon implantation of cancer cells or tumor tissue, animal models enable the undertaking of rigorous experimentation to understand the underlying mechanisms of cancer-induced cachexia. However, to date, it remains a major challenge to translate these results to humans, which is reflected by disappointing outcomes of numerous clinical trials in cachectic cancer patients. For example, several antibodies targeting the pro-cachectic cytokines tumor necrosis factoralpha (TNF- $\alpha$ ) and interleukin- 6 (IL-6) have been investigated in phase I and phase II clinical trials for the treatment of cancer cachexia (8-11). Both preclinical and clinical data have provided the conceptual basis for the development of these anti-cytokine strategies to treat cancer cachexia, however, so far, the majority of clinical trials evaluating these anti-cytokine therapies were not able to achieve positive outcomes (12).

In chapter 2, we described the generation and initial characterization of pancreatic tumor organoids that provide a novel platform for studying the crosstalk between the tumor and the host in human cancer-induced cachexia. We demonstrated that the established organoid cultures retain characteristic malignant features of the parental tumor and that the tumor organoids express variable levels of known cachexia-associated factors including IL-6, TNF- $\alpha$, IL-8, IL-1 $\alpha$, IL-1 $\beta$, Mcp-1, GDF15, and LIF. A major advantage of these primary tumor organoid cultures is that they can be efficiently generated from tumor resection material. This unique platform allows to perform a comprehensive pre-operative patient assessment of cachexia-related parameters (i.e. nutritional status, physical performance, body composition, and inflammation), which opens new opportunities to link in vitro findings to the cachexia status of the patient. To be able to implement primary tumor organoids in translational cancer cachexia research, there are two essential key elements that should be addressed:

1) Assessment of cachexia-related parameters. In chapter 2 , we have systematically assessed the cachexia status of each individual patient. According to the international consensus definition (1), we have classified patients as either cachectic or non-cachectic. Although this definition is mainly based on the percentage of weight loss, we have observed considerable heterogeneity among additional cachexia-related parameters, including the nutritional status, body composition, and biological markers. These interindividual differences underscore the heterogeneous presentation of cachexia. Therefore, thorough assessment of many cachexia-related parameters, as was performed in the current study, will be essential to get insight into the cachexia status of the patient and will ultimately aid in the translation of in vitro findings to specific aspects of the actual cachectic status of the patient.

2) Characterization of organoids vs parental tumor. In recent years, primary tumor organoids have been shown to closely recapitulate key pathophysiological aspects of pancreatic, 
colon, breast, and gastric cancer both in vitro and in vivo, supporting their applicability as a pre-clinical model to study diseases-specific mechanisms (13-16). In parallel, organoids can also be established from healthy epithelial tissues that also recapitulate the epithelial architecture and physiology of the tissue they were isolated from (17). In future studies, epithelial organoids from healthy tissue could be included as a non-tumor control group. For the implementation of healthy and tumor organoids in cancer cachexia research, it is essential to validate that organoids can be efficiently generated and they retain the physiological vs. pathophysiological aspects of the tissue of origin, as shown by histologic, genomic, and transcriptomic analyses.

Whereas the focus of this thesis is on pancreatic cancer-induced cachexia, the efficient generation of tumor organoids from primary tumor epithelial cells also allow the generation and characterization of tumor organoids from other cancer types that are often associated with cachexia. Although patients with pancreatic cancer have the highest prevalence of cancer cachexia ( 70\%), it is also present in a significant proportion of patients with colorectal ( $\sim 5 \%)$, lung ( 50\%), head and neck ( $\sim 5 \% \%)$, and gastro-esophageal cancer ( 60\%) (2). Since the discovery of the organoid technology in 2009 (18), numerous protocols have been developed for the successful generation of organoids from these cancer types $(15,19-21)$. The establishment of living organoid biobanks in combination with comprehensive assessment of cachexia-related parameters of each individual donor will open up new opportunities to further explore the cachexia-inducing properties of cancer cells.

A major limitation of the tumor organoid model is that it does not reflect the complex systemic interactions that contribute to the development of cachexia in cancer patients. Given the complex biology behind human cancer cachexia and the numerous variables that are playing a role (i.e. tumor biology and location, host-tumor interactions, co-morbidities, prior anti-cancer therapies, and psychosocial issues), organoids will also not be able to fully model the heterogeneous presentation of the cancer cachexia syndrome (22). Therefore, murine cancer models are still widely used to study cachexia-associated tumor-host interactions. Historically, murine cachexia research has relied on four models: Lewis lung carcinoma (LLC), C26 colorectal adenocarcinoma (C26), patient-derived tumor xenografts (PDX), and genetically engineered mouse models (GEMMs). In recent years, PDX have gained popularity for modeling human pancreatic cancer-induced cachexia (23-27). A major advantage of these models is the use of human pancreatic cancer cell lines or patientderived tissues that are transplanted into mice, which may better represent the tumor-host interactions that underlie the tissue wasting processes in cachectic cancer patients.

Primary pancreatic tumor organoids have also been successfully transplanted into mice and have been shown to recapitulate disease-related aspects, including the stages of PDA progression (13). Whereas the reproducibility of experiments using patient-derived tumor xenograft models is hampered by limited availability of tumor material, tumor 
organoids can be expanded long-term to increase the number of tumor cells and can therefore contribute to the establishment of more robust patient-derived tumor xenograft models. In addition, pre-operative assessment of cachexia-related parameters may enable to link these in vivo findings to the cachexia status of the patient. To test this hypothesis, we recently performed a pilot study in which we investigated whether pancreatic tumor organoid cultures from cachectic patients can induce systemic symptoms of cachexia. We transplanted two independently established organoid cultures into the flank of immunocompromised mice. Tumor organoids successfully engrafted in the flank of nude mice and induced local disease, as shown by a marked stromal reaction and similarities in the morphology between the parental tumor and the corresponding organoid grafts. More importantly, systemic symptoms associated with cachexia, such as reduced increases in body weight, were also observed. However, these systemic effects were only present in a limited number of mice. A major limitation of the establishment of human xenografts is the necessity of using immunocompromised mice to prevent rejection of the transplanted human cancer cells. These immunocompromised mice lack the interaction between the tumor and the immune system, which is suggested to significantly contribute to the cachexia-associated catabolic processes. Furthermore, the tumors are generally implanted in very young animals, which may result in the inhibition of weight gain rather than weight loss.

To conclude, it should be recognized that there is no single pre-clinical model can address all aspects of human cancer cachexia. It is therefore important to carefully consider these raised issues. Given the heterogeneity of cachexia, the use of tumor organoids in combination with a comprehensive pre-operative assessment of cachexia parameters will provide a novel exciting platform for translational cancer cachexia research.

\section{Tumor-host interactions in cancer cachexia}

It is widely assumed that tumor- and host-derived catabolic stimuli and pro-inflammatory cytokines promote tissue wasting in cancer cachexia. However, to date, relatively little is known about tumor-host crosstalk in human cancer cachexia. One long-standing theory holds that factors released from tumor cells promote muscle wasting by directly targeting skeletal muscle tissue. Data supporting this theory comes primarily from pre-clinical experimental models (3). A commonly used approach to study the direct effects of tumorderived factors on target tissues involves the treatment of cultured target cells with tumor cell-derived conditioned medium (CM). Although these studies have tremendously contributed to our current mechanistic understanding of cancer cachexia-associated muscle mass depletion, the translatability to the human situation remains questionable. In this section, I will discuss the use of patient-derived organoids to study the direct interaction between the tumor and the host, including the tumor-muscle crosstalk, which was the focus of the studies described in this thesis. 


\section{Tumor-muscle crosstalk}

The loss of skeletal muscle mass and function is a key characteristic of cancer cachexia that markedly impairs patient quality of life and survival. In addition to the progressive loss of skeletal muscle mass, also cardiac muscle wasting has recently been reported in cachectic cancer patients, suggesting that cardiac muscle may be similarly affected in cachectic cancer patients $(28,29)$. So far, it is not known whether the changes found in atrophying skeletal and cardiac muscle in response to tumor-derived factors also occur in smooth muscle, which is the third muscle compartment that fulfils a crucial role in human physiology.

Previous studies using CM from various tumor cell lines supports the notion that tumor cellderived factors promote skeletal muscle atrophy $(5,30,31)$. In chapter 2, we already showed that organoids from cachectic patients express higher levels of cachexia-associated factors compared to organoids from non-cachectic patients. This raised the question whether these tumor-derived factors could directly induce skeletal muscle wasting. Against our expectations, and in contrast to numerous in vitro and in vivo studies, the human pancreatic tumor organoid secretome did not induce skeletal muscle atrophy signaling in mature $\mathrm{C} 2 \mathrm{C} 12$ myotubes. Nevertheless, in support of our results, similar observations have recently been reported: Guigni et al. investigated the effect of human lung tumor primary cell line-derived $\mathrm{CM}$ on mature $\mathrm{C} 2 \mathrm{C} 12$ myotubes and also did not detect differences in myosin content and myotube size compared to the control (3). In addition, the patientderived tumor organoid secretome also did not induce atrophy signaling in smooth muscle cells (SMCs) (chapter 5). These data suggest that other processes than activation of the proteasome by E3 ubiquitin ligases may be triggered by the tumor secretome and contribute to the loss of muscle mass in human cancer-induced cachexia.

Maintenance of muscle mass is coordinated by a balance between the activation and inhibition of catabolic and anabolic signaling pathways (32). Loss of muscle mass occurs when the balance shifts to an increase in muscle protein breakdown and/or a decrease in muscle protein synthesis. Interestingly, along with the absence of muscle atrophy signaling in response to the tumor organoid secretome, we also did not detect inhibition of protein signaling pathways, as assessed by REDD1 expression (chapter 3). REDD1, which is a repressor of protein synthesis signaling, has previously been shown to be elevated in skeletal muscle during various atrophic conditions $(33,34)$. Thus far, our in vitro data does not support the hypothesis that tumor-derived factors activate catabolic signaling and/or inhibit protein synthesis in cultured $\mathrm{C} 2 \mathrm{C} 12$ myotubes. To confirm these results in future studies, the use of radioactive tracer isotopes is particularly suited to monitor muscle protein metabolism (35). Numerous studies have measured muscle protein synthesis rates using stable isotope tracers of amino acids (36-38). The incorporation of labelled amino acids into muscle protein allows for the calculation of a fractional synthetic rate (39). Using the radioactive isotope tracer method, we recently showed that ascites of sarcopenic patients induced pronounced skeletal muscle protein metabolism changes in $\mathrm{C} 2 \mathrm{C} 12$ cells 
that correlate with clinical muscle measures of the patient (unpublished). However, in contrast to the tumor organoid secretome, ovarian cancer ascites is a complex reservoir of both tumor-derived and systemic factors and therefore, we cannot distinguish whether the muscle protein changes were induced by tumor-derived or systemic factors, or both.

Another important process in the maintenance of muscle mass is myogenesis, the formation of muscular tissue. Impaired myogenesis and muscle regeneration have previously been observed in multiple muscle pathologies, including cancer-associated cachexia (40-44). Nevertheless, in chapter 3, we observed that the pancreatic tumor organoid secretome transiently increased the proliferation of $\mathrm{C} 2 \mathrm{C} 12$ myoblasts and accelerated their alignment and fusion rather than repressing these processes. These alterations were accompanied by the increased expression of muscle-specific and myogenic fusion genes. These data indicate that pancreatic tumor organoid-derived factors promote myogenic commitment of muscle progenitor cells at the expense of satellite repopulation, which may eventually contribute to impaired muscle mass maintenance in cancer cachexia. Satellite cells are normally quiescent but can become activated in response to muscle damage. Upon activation, these cells enter the cell cycle and give rise to proliferative satellite-cell progeny, called myoblasts. In the context of cancer cachexia, it has previously been shown that muscle damage triggers satellite cell activation, resulting in the repopulation of Pax7-positive satellite cells $(41,43)$. Upon entering the myogenic program, myoblasts committing to terminal differentiation lose Pax-7 expression, whereas Pax-7 expression is maintained by myoblasts that become quiescent to replete the satellite cell population. The reduced Pax-7 expression in myoblast cultures in response to the tumor organoid secretome that we observed may therefore reflect a depletion of the population of replication competent cells, referred to as 'reserve cells'. In vivo, such a depletion of muscle precursor cells will interfere with long term myogenic potential, ultimately contributing to muscle mass loss. The transplantation of patient-derived organoids into mice allows to further explore this hypothesis in the in vivo setting.

Contractile SMCs are the force-producing cells essential for gut motor activity required to move nutrients through the gastrointestinal (GI)-tract (45). Diminished contractile SMC function results in GI-motility problems that may contribute to the underlying nutritionalrelated complications in cancer cachexia. In support of the hypothesis that cachexiaassociated muscle alterations may not only be limited to striated muscle, Zhou et al. recently identified Gl-related symptoms of early satiety, diarrhea, and constipation more often in cachectic cancer patients compared to non-cachectic patients (46). In addition, patients with pancreatic cancer often present with nausea and vomiting in the absence of mechanical obstruction (47). Particularly in the context of the cachexia syndrome that affect nutrition and fluid-electrolyte balance in cancer patients, it is important to recognize and treat these symptoms timely. If unrecognized and untreated, malignancy-associated GImotility problems can compound the cachexia syndrome. The potential consequences 
include chronic nausea and vomiting, electrolyte disturbances, dehydration, hospitalization, and significant impairment of quality of life.

In contrast to skeletal and cardiac muscle cells that differentiate terminally, mature contractile smooth muscle cells retain remarkable plasticity as expressed by their ability to undergo so-called phenotypic modulation in response to injury and disease. Given the ability of SMCs to adapt their phenotype in response to a variety of stimuli, we first established a human visceral SMC culture system that allows controlled phenotypic modulation toward both the synthetic and contractile phenotype (chapter 4). In line with the effects of the tumor organoid secretome on skeletal muscle cells, we reported similar effects on SMCs (chapter 5): the tumor organoid secretome markedly accelerated the proliferation rate of SMCs which was accompanied by reduced expression of SMC contractile specific proteins.

In chapter 6, we investigated whether a low skeletal muscle mass in patients with pancreatic cancer was associated with aberrations of their intestinal smooth musculature. We observed reduced expression of the contractile SMC-specific protein smoothelin, which may reflect deficiencies of smooth muscle contractile function. The pronounced collagen deposition around the myenteric plexus of sarcopenic patients corroborates this notion and suggests that regulation of smooth muscle contractility may also be compromised in sarcopenic patients. Although these data show that smooth muscle may be a target in the cancer cachexia syndrome, data regarding GI-related symptoms and functionality of the Gltract are lacking. The gastrointestinal symptom rating scale (GSRS) is a validated questionnaire used by clinicians to score $\mathrm{Gl}$ symptoms reported by patients $(48,49)$. This questionnaire could easily be implemented in the screening of cachectic cancer patients and will provide insight into the prevalence of these symptoms. Furthermore, accurately measuring the complex motor behaviors of the GI-tract has tremendous value in broadening our understanding of the physiology and pathophysiology of the Gl-tract in cachectic cancer patients. Tests that are currently available for motility measurements include scintigraphy (50), stable isotope breath tests (51), and wireless $\mathrm{pH}$ and motility capsules $(52,53)$. Scintigraphy is the most widely used test for the assessment of gastric motility and provides a direct, non-invasive quantification of gastric emptying. The main indications for use of this test are the investigation of unexplained nausea, vomiting, and dyspeptic symptoms. The $\mathrm{pH}$ and motility capsule is particularly relevant for the assessment of GI-motility in cachectic patients $(52,54,55)$. It is a nondigestible wireless capsule that can measure the $\mathrm{pH}$, pressure, and temperature throughout the Gl-tract. The capsule acquires data continuously for up to 5 days, permitting calculation of gastric, small-bowel, colon, and whole-gut transit. Major advantages of the motility capsule are that the study can be conducted anywhere, there is a lack of radioactivity, and that it has the ability to determine small-bowel, colon, and whole-gut transit times as well as contractility of separate gut segments. The use of the wireless motility capsule in well-phenotyped 
cachectic and non-cachectic patients will enable accurate and objective assessment of cachexia-associated GI-motility dysfunction.

\section{Tumor-immune crosstalk}

To fully understand the etiology of cancer cachexia, it is essential to identify the mediators that drive the communication between the tumor and the host. Aside from the tumorderived factors that are suggested to be involved in the tumor-muscle crosstalk, tumorinduced inflammation has also been suggested to be an important factor in the pathophysiology of cancer cachexia. Systemic inflammation is a hallmark of cancer and has been shown to be associated with muscle wasting and fat depletion in cancer cachexia (56). Moreover, the inflammatory response has been suggested to be the main driving force behind the metabolic alterations present in cancer-induced cachexia $(57,58)$. Systemic inflammation in cancer patients can have multiple origins; cytokines and other inflammatory mediators can be released by tumor cells and host tissue, including activated immune cells and cancer-associated fibroblasts. Numerous pro-inflammatory cytokines have been shown to be generated through tumor-immune cell crosstalk. These cytokines may act directly as well as indirectly on target tissues (e.g. skeletal and cardiac muscle, adipose tissue) through alteration of central nervous system controls of energy intake and expenditure, resulting in anorexia, weight loss, and skeletal muscle atrophy (2).

Although it is well-appreciated that inflammation is a major driver of cancer cachexia, to date, only little is known about the specifics of tumor-immune system crosstalk in cachectic cancer patients. Again, patient-derived organoids from well-phenotyped cachectic and non-cachectic patients could be particularly useful to gain in-depth knowledge on the interaction between tumor cells and immune cells. First of all, similarly to the previously mentioned CM transfer experiments using muscle cells, cultured immune cells could be treated with the tumor organoid-derived secretome to assess the direct effects of tumor released factors on immune activation. Immune cells play a key role in the host defense against cancer. Upon encountering danger signals, these cells undergo activation leading to a modulation of their functions. Recent studies also show that upon activation, immune cells show distinct metabolic changes that impact their effector functions $(59,60)$. In a recent pilot experiment, we treated human macrophages with the tumor organoid secretome. Preliminary results revealed significantly decreased basal macrophage oxygen consumption rates in response to tumor organoids $\mathrm{CM}$ from cachectic patients compared to $\mathrm{CM}$ from non-cachectic patients. Also, the spare respiratory capacity, which indicates the capability of the cell to respond to an energetic demand, as well as how closely the cell is respiring to its theoretical maximum, appeared to be reduced. These data may indicate that tumor-derived factors from cachectic patients impair the mitochondrial oxidative phosphorylation capacity. Alternatively, organoids could be co-cultured with immune cells, which will greatly contribute to our current understanding on the interaction of tumor cells and immune cells and how this can impact the systemic consequences of the 
cancer cachexia syndrome. Organoid cultures typically contain exclusively neoplastic epithelium and lack the immune cells that are present in the tumor microenvironment. However, recent efforts have resulted in the establishment of co-culture systems with organoids and immune cells (61-63). For example, Dijkstra et al. have established a strategy to obtain tumor-reactive $\mathrm{T}$ cell populations from peripheral blood by organoid co-culture (61). This model system can be used to study individualized interactions between tumor cells and matched immune cells from the donor patient.

\section{Additional cachexia-associated target tissues}

Even though cancer cachexia is particularly characterized by the loss of skeletal muscle mass and a systemic inflammatory response, several other organs and tissues are also affected, making this condition a true multi-organ syndrome (57). For example, in addition to skeletal muscle, a substantial portion of weight loss in cancer patients derives from the depletion of adipose tissue. Previous studies have shown that the depletion of fat mass predominantly results from lipolysis and browning of white adipose tissue (WAT) rather than the irreversible degeneration of fat cells (64-66). These effects may be directly induced by tumor-derived factors, as has previously been shown by in vitro studies $(67,68)$. Furthermore, an increasing body of evidence suggests that hypothalamic inflammation is linked to the systemic inflammatory response and that hypothalamic inflammation is involved in the regulation of energy intake and expenditure in cachexia (69-71). Hypothalamic exposure to pro-inflammatory stimuli such as IL-1 $\beta$ and TNF-alpha triggers an acute illness response, leading to anorexia, weight loss, and skeletal muscle atrophy (72). To further expand our understanding of the underlying mechanisms of this multi-organ syndrome, tumor organoids from cachectic and non-cachectic patients can also be used to investigate the direct effects of tumor-derived factors on adipocytes and the hypothalamus in both in vitro and in vivo experiments.

\section{Identification of cachexia-inducing factors in human cancer cachexia}

To date, efforts to identify mediators and potential biomarkers of human cancer cachexia have been centered on the levels of circulating factors that have been associated with the cachexia syndrome (73-78). However, it remains unclear whether these circulating factors are derived from the host tissues or from the tumor. In addition, the extent to which these cachexia-associated secreted factors are expressed by different tumor types and how these factors contribute to the development of the cachexia syndrome have yet to be determined.

In chapter 2, we have already shown that pancreatic tumor organoids express variable levels of known cachexia-associated factors including IL-6, TNF- $\alpha$, IL-8, IL-1 $\alpha$, IL-1 $\beta$, Mcp-1, GDF15, and LIF. However, as a consequence of the relatively small number of organoid cultures studied, the heterogeneous presentation of the cachexia syndrome, and 
the lack of distinctive diagnostic criteria that can stratify patients according to the actual cachexia status, the significance of the observed increases and decreases of cachexiarelated factors in organoids remains unclear. These data should therefore be validated using a larger number of organoids cultures established from well-phenotyped cachectic and noncachectic patients. Ultimately, this will enable more robust analyses of the differences in expression of cachexia-inducing factors by organoids from cachectic and non-cachectic patients.

The identification of tumor factors that drive key processes underlying cachexiarelated metabolic changes also requires the use of more advanced technological approaches. In recent years, omics- and systems biology approaches have gained popularity in cancer cachexia research. For example, transcriptomic approaches can be applied to identify genes encoding secreted protein $(79,80)$. Using publicly available RNA sequencing data sets of human cancers, Freire et al. recently characterized the molecular landscape of potential cachexia-inducing factors in human cancers with different prevalences of cachexia (81). Interestingly, this study showed that a specific tumor expression profile consisting of 25 potential cachexia-inducing factors was significantly associated with a poor prognosis and correlated with the prevalence of cachexia in cancer patients. Although in silico-based approaches have become useful tools for profiling the genes encoding secreted proteins in cancer research, the usual absence of cachexia-related clinical data impedes the analysis of tumor expression levels of these cachexia-inducing factor genes in relation to the cachexia status (81). In addition, human tumor tissue used to generate RNA-sequencing data does not only contain a pure population of tumor cells but also other cell types that are present in the tumor microenvironment, including stromal cells and immune cells. Organoid cultures consist purely of epithelial cells and therefore allow direct gene expression analysis without contamination from other cell subsets that are present in the TME. Single-cell RNA sequencing may be an alternative approach that can be used to differentiate the specific contributions of different cell types in the TME to the tumor expression profile of cachectic patients.

The tumor secretome is considered to be a valuable reservoir of potential biomarkers and disease mediators in cancer. Whereas transcriptomics can be used to analyze the complete set of messenger RNA molecules produced in a population of cells, proteomic-based approaches enable the analysis of proteins that are secreted by these cells. Although proteomic approaches have often been used to profile the cancer secretome (82-85), these studies generally involved the analysis of 2D cancer cell line-derived conditioned media. As already discussed previously, it is unlikely that established human cancer cell lines that have been cultured for decades harbor clinically relevant cachectic properties. The complete absence of cachexia-related clinical data further impairs the use of established human pancreatic cancer cell lines for the identification of secreted cachexiainducing factors. Again, the tumor organoid secretome may contain a more representative reservoir of potential cachexia-inducing factors whose expression levels can be correlated 
to a variety of cachexia-associated parameters. In parallel, the circulating levels of these factors could be measured in the blood of the same patients.

In the context of tumor-host crosstalk, tumor-derived extracellular vesicles (EVs) have also emerged as potential mediators of cell-cell communication between tumor and host cells. EVs are membrane-enclosed particles secreted by various cell types into the extracellular surrounding enabling intercellular communication. The function of these EVs appears to be mainly dependent on the cell type from which they originate and the cargo they carry (86). EVs can contain biologically active molecules, including proteins (e.g. cytokines), nucleic acids (e.g. mRNA, miRNA, DNA), and lipids (e.g. ceramides). Thus far, only a limited number of studies have been published on the potential effect of tumor-released EVs on cachexiaassociated target tissues. In 2014, He et al. were the first to show that tumor-derived vesicles induces apoptosis of skeletal muscle cells (87). More recently, Zhang et al. reported similar findings. Diverse types of cachexia-inducing tumor cells constitutively released high levels of extracellular Hsp70 and Hsp90 through EVs, and tumor cell-released extracellular Hsp70 and Hsp90 stimulated muscle catabolism (6). Whether patient-derived organoids from cachectic patients release EVs that drive key processes underlying cancer-induced cachexia in relevant target cells remains elusive.

\section{Assessing cancer cachexia status}

Diagnosis of cancer cachexia remains a challenge both in research and in clinical practice. However, to be able translate novel findings into clinically meaning full results, it is essential to assess the actual cachexia status of patients involved in research. Although the international consensus definition provides useful guidelines for the assessment and classification of the cachexia status, we still frequently encountered difficulties in categorizing patients into cachectic vs non-cachectic groups.

To date, involuntary weight loss of $\geq 5 \%$ in the preceding 6 months is still the most widely used diagnostic criterium to diagnose cachexia in cancer patients. This is also not surprising given that involuntary weight loss is a key characteristic of this wasting syndrome. Nevertheless, although body-weight measurements can easily be implemented in the outpatient clinic, accurate body weight data over the past 6 months are often lacking; body weight is usually well documented at the time of diagnosis, but reliable data on patient's body weight 6 months ago are often not available. Furthermore, it should be recognized that body weight measurements cannot differentiate between skeletal muscle and fat tissue mass and may therefore underestimate actual lean body mass loss in overweight or obese patients and in those that have gained weight because of a growing tumor mass or by abnormal accumulation of fluid within the body (i.e. edema, ascites) (88). Recently, Vanhoutte et al. compared two definitions of cachexia using different diagnostic guidelines applied on the same patient population and showed that putting the focus on weight loss 
overrates the assignment of the diagnosis of cachexia resulting in survival rates with less prognostic value (89). Altogether, this underscores the need for additional accurate measurements rather than using weight-loss measurements alone.

In contrast to body weight measurements, computed-tomography (CT)-based body composition analysis is not influenced by tumor load, edema, or ascites and can differentiate between individual tissue compartments (e.g. skeletal muscle mass, adipose tissue, bone, and organs) (90). Of particular interest is the cross-sectional muscle area at the third lumbar vertebra (L3), which is a good estimate of whole-body muscularity (skeletal muscle index, SMI). Also, the L3-SMI has been shown to be a surrogate marker of sarcopenia and cachexia and has prognostic value in terms of patient survival and post-operative morbidity $(1,91-93)$. Due to the increasing use of CT-imaging for cancer staging, CT-scans are widely available to quantify muscle mass in cancer patients. These CT-scans are often retrospectively collected and used to investigate possible associations between body composition parameters and cachexia-associated characteristics. However, as a consequence of the retrospective design of these studies, accurate data of relevant cachexia-related parameters are often missing (e.g. weight loss, nutritional status, Creactive protein and other markers of inflammation). In chapter 6 , we have used the L3-SMI to assign patients to either a high L3-SMI or low L3-SMI group. This enabled us to investigate whether the intestinal smooth musculature was affected in patients with a low skeletal muscle mass compared to patients with a high skeletal muscle mass, which is suggested to reflect patients with or without cachexia, respectively. However, although CT-imaging analysis is considered a highly valuable tool to accurately assess body composition, it is important to recognize that single baseline measurements do not capture the dynamic changes of muscle loss. Measuring body composition at different time points will therefore be of added value to assess muscle loss over time. Given these limitations and challenges in classifying patients either cachectic or non-cachectic, we implemented a systematic approach of assessing the cachexia status of patients before establishing and characterizing tumor organoid cultures (chapter 2).

\section{Concluding remarks}

To conclude, the underlying mechanisms of cancer-induced cachexia remain poorly understood, hampering the successful management of this complex clinical condition. Novel mechanistic insights are therefore urgently needed. This thesis demonstrated that patient-derived tumor organoids can provide a useful platform for translational cancer cachexia research. The use of tumor organoids in combination with a comprehensive preoperative assessment of cachexia parameters of donor patients will enable us to link in vitro findings to the cachexia status of the patient. Given the heterogeneous presentation of the cachexia syndrome and the lack of distinctive diagnostic criteria that can stratify patients according to the actual cachexia status, it should be considered to expand the number of organoid cultures established from well-phenotyped cachectic and non-cachectic patients. 
This will enable more robust analyses of the inter-individual differences in both in vitro and in vivo studies. Gaining novel insights into the mechanisms of cancer-induced cachexia may ultimately aid in the identification of predictive and prognostic biomarkers and the development of effective treatment regimens. 


\section{References}

1. Fearon K, Strasser F, Anker SD, Bosaeus I, Bruera E, Fainsinger RL, et al. Definition and classification of cancer cachexia: an international consensus. Lancet Oncol. 2011;12(5):48995.

2. Baracos VE, Martin L, Korc M, Guttridge DC, Fearon KCH. Cancer-associated cachexia. Nat Rev Dis Primers. 2018;4:17105.

3. Guigni BA, van der Velden J, Kinsey CM, Carson JA, Toth MJ. Effects of conditioned media from murine lung cancer cells and human tumor cells on cultured myotubes. Am J Physiol Endocrinol Metab. 2020;318(1):E22-E32.

4. Jackman RW, Floro J, Yoshimine R, Zitin B, Eiampikul M, El-Jack K, et al. Continuous Release of Tumor-Derived Factors Improves the Modeling of Cachexia in Muscle Cell Culture. Front Physiol. 2017;8:738.

5. Seto DN, Kandarian SC, Jackman RW. A Key Role for Leukemia Inhibitory Factor in C26 Cancer Cachexia. J Biol Chem. 2015;290(32):19976-86.

6. Zhang G, Liu Z, Ding H, Zhou Y, Doan HA, Sin KWT, et al. Tumor induces muscle wasting in mice through releasing extracellular Hsp70 and Hsp90. Nat Commun. 2017;8(1):589.

7. Ben-David U, Siranosian B, Ha G, Tang H, Oren Y, Hinohara K, et al. Genetic and transcriptional evolution alters cancer cell line drug response. Nature. 2018;560(7718):32530.

8. Bayliss TJ, Smith JT, Schuster M, Dragnev KH, Rigas JR. A humanized anti-IL-6 antibody (ALD518) in non-small cell lung cancer. Expert Opin Biol Ther. 2011;11(12):1663-8.

9. Jatoi A, Dakhil SR, Nguyen PL, Sloan JA, Kugler JW, Rowland KM, Jr., et al. A placebocontrolled double blind trial of etanercept for the cancer anorexia/weight loss syndrome: results from NOOC1 from the North Central Cancer Treatment Group. Cancer. 2007;110(6):1396-403.

10. Jatoi A, Ritter HL, Dueck A, Nguyen PL, Nikcevich DA, Luyun RF, et al. A placebo-controlled, double-blind trial of infliximab for cancer-associated weight loss in elderly and/or poor performance non-small cell lung cancer patients (N01C9). Lung Cancer. 2010;68(2):234-9.

11. Wiedenmann B, Malfertheiner P, Friess H, Ritch P, Arseneau J, Mantovani G, et al. A multicenter, phase II study of infliximab plus gemcitabine in pancreatic cancer cachexia. $J$ Support Oncol. 2008;6(1):18-25.

12. Prado BL, Qian Y. Anti-cytokines in the treatment of cancer cachexia. Ann Palliat Med. 2019;8(1):67-79.

13. Boj SF, Hwang Cl, Baker LA, Chio, II, Engle DD, Corbo V, et al. Organoid models of human and mouse ductal pancreatic cancer. Cell. 2015;160(1-2):324-38.

14. Sachs N, de Ligt J, Kopper O, Gogola E, Bounova G, Weeber F, et al. A Living Biobank of Breast Cancer Organoids Captures Disease Heterogeneity. Cell. 2018;172(1-2):373-86 e10.

15. van de Wetering M, Francies HE, Francis JM, Bounova G, lorio F, Pronk A, et al. Prospective derivation of a living organoid biobank of colorectal cancer patients. Cell. 2015;161(4):93345.

16. Yan HHN, Siu HC, Law S, Ho SL, Yue SSK, Tsui WY, et al. A Comprehensive Human Gastric Cancer Organoid Biobank Captures Tumor Subtype Heterogeneity and Enables Therapeutic Screening. Cell Stem Cell. 2018;23(6):882-97 e11.

17. Clevers H. Modeling Development and Disease with Organoids. Cell. 2016;165(7):1586-97.

18. Sato T, Vries RG, Snippert HJ, van de Wetering M, Barker N, Stange DE, et al. Single Lgr5 stem cells build crypt-villus structures in vitro without a mesenchymal niche. Nature. 2009;459(7244):262-5. 
19. Driehuis E, Spelier S, Beltran Hernandez I, de Bree R, S MW, Clevers H, et al. Patient-Derived Head and Neck Cancer Organoids Recapitulate EGFR Expression Levels of Respective Tissues and Are Responsive to EGFR-Targeted Photodynamic Therapy. J Clin Med. 2019;8(11).

20. Li X, Francies HE, Secrier M, Perner J, Miremadi A, Galeano-Dalmau N, et al. Organoid cultures recapitulate esophageal adenocarcinoma heterogeneity providing a model for clonality studies and precision therapeutics. Nat Commun. 2018;9(1):2983.

21. Sachs N, Papaspyropoulos A, Zomer-van Ommen DD, Heo I, Bottinger L, Klay D, et al. Longterm expanding human airway organoids for disease modeling. EMBO J. 2019;38(4).

22. Fearon K, Arends J, Baracos V. Understanding the mechanisms and treatment options in cancer cachexia. Nat Rev Clin Oncol. 2013;10(2):90-9.

23. Delitto D, Judge SM, Delitto AE, Nosacka RL, Rocha FG, DiVita BB, et al. Human pancreatic cancer xenografts recapitulate key aspects of cancer cachexia. Oncotarget. 2017;8(1):117789.

24. Henderson SE, Makhijani N, Mace TA. Pancreatic Cancer-Induced Cachexia and Relevant Mouse Models. Pancreas. 2018;47(8):937-45.

25. Jones-Bolin S, Ruggeri B. Orthotopic model of human pancreatic ductal adenocarcinoma and cancer cachexia in nude mice. Curr Protoc Pharmacol. 2007;Chapter 14:Unit 143.

26. Michaelis KA, Zhu X, Burfeind KG, Krasnow SM, Levasseur PR, Morgan TK, et al. Establishment and characterization of a novel murine model of pancreatic cancer cachexia. J Cachexia Sarcopenia Muscle. 2017;8(5):824-38.

27. Togashi $Y$, Kogita A, Sakamoto H, Hayashi H, Terashima M, de Velasco MA, et al. Activin signal promotes cancer progression and is involved in cachexia in a subset of pancreatic cancer. Cancer Lett. 2015;356(2 Pt B):819-27.

28. Barkhudaryan A, Scherbakov N, Springer J, Doehner W. Cardiac muscle wasting in individuals with cancer cachexia. ESC Heart Fail. 2017;4(4):458-67.

29. Springer J, Tschirner A, Haghikia A, von Haehling S, Lal H, Grzesiak A, et al. Prevention of liver cancer cachexia-induced cardiac wasting and heart failure. Eur Heart J. 2014;35(14):932-41.

30. Gao S, Carson JA. Lewis lung carcinoma regulation of mechanical stretch-induced protein synthesis in cultured myotubes. Am J Physiol Cell Physiol. 2016;310(1):C66-79.

31. Sun R, Zhang S, Hu W, Lu X, Lou N, Yang Z, et al. Valproic acid attenuates skeletal muscle wasting by inhibiting C/EBPbeta-regulated atrogin1 expression in cancer cachexia. Am J Physiol Cell Physiol. 2016;311(1):C101-15.

32. Schiaffino S, Dyar KA, Ciciliot S, Blaauw B, Sandri M. Mechanisms regulating skeletal muscle growth and atrophy. FEBS J. 2013;280(17):4294-314.

33. Britto FA, Cortade F, Belloum Y, Blaquiere M, Gallot YS, Docquier A, et al. Glucocorticoiddependent REDD1 expression reduces muscle metabolism to enable adaptation under energetic stress. BMC Biol. 2018;16(1):65.

34. Gordon BS, Williamson DL, Lang CH, Jefferson LS, Kimball SR. Nutrient-induced stimulation of protein synthesis in mouse skeletal muscle is limited by the mTORC1 repressor REDD1. J Nutr. 2015;145(4):708-13.

35. Brook MS, Wilkinson DJ. Contemporary stable isotope tracer approaches: Insights into skeletal muscle metabolism in health and disease. Exp Physiol. 2020;105(7):1081-9.

36. Rennie MJ, Edwards RH, Halliday D, Matthews DE, Wolman SL, Millward DJ. Muscle protein synthesis measured by stable isotope techniques in man: the effects of feeding and fasting. Clin Sci (Lond). 1982;63(6):519-23.

37. van Dijk DPJ, Horstman AMH, Smeets JSJ, den Dulk M, Grabsch HI, Dejong CHC, et al. Tumour-specific and organ-specific protein synthesis rates in patients with pancreatic cancer. J Cachexia Sarcopenia Muscle. 2019;10(3):549-56.

38. Wilkinson DJ, Brook MS, Smith K, Atherton PJ. Stable isotope tracers and exercise physiology: past, present and future. J Physiol. 2017;595(9):2873-82. 
39. Carson BP, Patel B, Amigo-Benavent M, Pauk M, Kumar Gujulla S, Murphy SM, et al. Regulation of muscle protein synthesis in an in vitro cell model using ex vivo human serum. Exp Physiol. 2018;103(6):783-9.

40. Arneson PC, Doles JD. Impaired Muscle Regeneration in Cancer-Associated Cachexia. Trends Cancer. 2019;5(10):579-82.

41. He WA, Berardi E, Cardillo VM, Acharyya S, Aulino P, Thomas-Ahner J, et al. NF-kappaBmediated Pax7 dysregulation in the muscle microenvironment promotes cancer cachexia. $J$ Clin Invest. 2013;123(11):4821-35.

42. Hogan KA, Cho DS, Arneson PC, Samani A, Palines P, Yang Y, et al. Tumor-derived cytokines impair myogenesis and alter the skeletal muscle immune microenvironment. Cytokine. 2018;107:9-17.

43. Inaba S, Hinohara A, Tachibana M, Tsujikawa K, Fukada SI. Muscle regeneration is disrupted by cancer cachexia without loss of muscle stem cell potential. PLoS One. 2018;13(10):e0205467.

44. Penna F, Costamagna D, Fanzani A, Bonelli G, Baccino FM, Costelli P. Muscle wasting and impaired myogenesis in tumor bearing mice are prevented by ERK inhibition. PLoS One. 2010;5(10):e13604.

45. Sanders KM, Koh SD, Ro S, Ward SM. Regulation of gastrointestinal motility--insights from smooth muscle biology. Nat Rev Gastroenterol Hepatol. 2012;9(11):633-45.

46. Zhou T, Yang K, Thapa S, Liu H, Wang B, Yu S. Differences in Symptom Burden Among Cancer Patients With Different Stages of Cachexia. J Pain Symptom Manage. 2017;53(5):919-26.

47. Leung J, Silverman W. Diagnostic and therapeutic approach to pancreatic cancer-associated gastroparesis: literature review and our experience. Dig Dis Sci. 2009;54(2):401-5.

48. Revicki DA, Wood M, Wiklund I, Crawley J. Reliability and validity of the Gastrointestinal Symptom Rating Scale in patients with gastroesophageal reflux disease. Qual Life Res. 1998;7(1):75-83.

49. Svedlund J, Sjodin I, Dotevall G. GSRS--a clinical rating scale for gastrointestinal symptoms in patients with irritable bowel syndrome and peptic ulcer disease. Dig Dis Sci. 1988;33(2):12934.

50. Abell TL, Camilleri M, Donohoe K, Hasler WL, Lin HC, Maurer AH, et al. Consensus recommendations for gastric emptying scintigraphy: a joint report of the American Neurogastroenterology and Motility Society and the Society of Nuclear Medicine. J Nucl Med Technol. 2008;36(1):44-54.

51. Szarka LA, Camilleri M, Vella A, Burton D, Baxter K, Simonson J, et al. A stable isotope breath test with a standard meal for abnormal gastric emptying of solids in the clinic and in research. Clin Gastroenterol Hepatol. 2008;6(6):635-43 e1.

52. Kuo B, McCallum RW, Koch KL, Sitrin MD, Wo JM, Chey WD, et al. Comparison of gastric emptying of a nondigestible capsule to a radio-labelled meal in healthy and gastroparetic subjects. Aliment Pharmacol Ther. 2008;27(2):186-96.

53. Lee AA, Rao S, Nguyen LA, Moshiree B, Sarosiek I, Schulman MI, et al. Validation of Diagnostic and Performance Characteristics of the Wireless Motility Capsule in Patients With Suspected Gastroparesis. Clin Gastroenterol Hepatol. 2019;17(9):1770-9 e2.

54. Rao SS, Camilleri M, Hasler WL, Maurer AH, Parkman HP, Saad R, et al. Evaluation of gastrointestinal transit in clinical practice: position paper of the American and European Neurogastroenterology and Motility Societies. Neurogastroenterol Motil. 2011;23(1):8-23.

55. Sharma A, Coles M, Parkman HP. Gastroparesis in the 2020s: New Treatments, New Paradigms. Curr Gastroenterol Rep. 2020;22(5):23.

56. Argiles JM, Busquets S, Stemmler B, Lopez-Soriano FJ. Cancer cachexia: understanding the molecular basis. Nat Rev Cancer. 2014;14(11):754-62.

57. Argiles JM, Stemmler B, Lopez-Soriano FJ, Busquets S. Inter-tissue communication in cancer cachexia. Nat Rev Endocrinol. 2018;15(1):9-20. 
58. McMillan DC. Systemic inflammation, nutritional status and survival in patients with cancer. Curr Opin Clin Nutr Metab Care. 2009;12(3):223-6.

59. Biswas SK. Metabolic Reprogramming of Immune Cells in Cancer Progression. Immunity. 2015;43(3):435-49.

60. Singer K, Cheng WC, Kreutz M, Ho PC, Siska PJ. Immunometabolism in cancer at a glance. Dis Model Mech. 2018;11(8).

61. Dijkstra KK, Cattaneo CM, Weeber F, Chalabi M, van de Haar J, Fanchi LF, et al. Generation of Tumor-Reactive T Cells by Co-culture of Peripheral Blood Lymphocytes and Tumor Organoids. Cell. 2018;174(6):1586-98 e12.

62. Neal JT, Li X, Zhu J, Giangarra V, Grzeskowiak CL, Ju J, et al. Organoid Modeling of the Tumor Immune Microenvironment. Cell. 2018;175(7):1972-88 e16.

63. Tsai S, McOlash L, Palen K, Johnson B, Duris C, Yang Q, et al. Development of primary human pancreatic cancer organoids, matched stromal and immune cells and 3D tumor microenvironment models. BMC Cancer. 2018;18(1):335.

64. Han J, Meng Q, Shen L, Wu G. Interleukin- 6 induces fat loss in cancer cachexia by promoting white adipose tissue lipolysis and browning. Lipids Health Dis. 2018;17(1):14.

65. Ryden M, Arner P. Fat loss in cachexia--is there a role for adipocyte lipolysis? Clin Nutr. 2007;26(1):1-6.

66. Zuijdgeest-van Leeuwen SD, van den Berg JW, Wattimena JL, van der Gaast A, Swart GR, Wilson $\mathrm{JH}$, et al. Lipolysis and lipid oxidation in weight-losing cancer patients and healthy subjects. Metabolism. 2000;49(7):931-6.

67. Elattar S, Dimri M, Satyanarayana A. The tumor secretory factor ZAG promotes white adipose tissue browning and energy wasting. FASEB J. 2018;32(9):4727-43.

68. Hu W, Ru Z, Zhou Y, Xiao W, Sun R, Zhang S, et al. Lung cancer-derived extracellular vesicles induced myotube atrophy and adipocyte lipolysis via the extracellular IL-6-mediated STAT3 pathway. Biochim Biophys Acta Mol Cell Biol Lipids. 2019;1864(8):1091-102.

69. Burfeind KG, Michaelis KA, Marks DL. The central role of hypothalamic inflammation in the acute illness response and cachexia. Semin Cell Dev Biol. 2016;54:42-52.

70. Cai D, Liu T. Hypothalamic inflammation: a double-edged sword to nutritional diseases. Ann N Y Acad Sci. 2011;1243:E1-39.

71. Molfino A, lannace A, Colaiacomo MC, Farcomeni A, Emiliani A, Gualdi G, et al. Cancer anorexia: hypothalamic activity and its association with inflammation and appetiteregulating peptides in lung cancer. J Cachexia Sarcopenia Muscle. 2017;8(1):40-7.

72. Braun TP, Zhu X, Szumowski M, Scott GD, Grossberg AJ, Levasseur PR, et al. Central nervous system inflammation induces muscle atrophy via activation of the hypothalamic-pituitaryadrenal axis. J Exp Med. 2011;208(12):2449-63.

73. Lerner L, Hayes TG, Tao N, Krieger B, Feng B, Wu Z, et al. Plasma growth differentiation factor 15 is associated with weight loss and mortality in cancer patients. J Cachexia Sarcopenia Muscle. 2015;6(4):317-24.

74. Lerner L, Tao J, Liu Q, Nicoletti R, Feng B, Krieger B, et al. MAP3K11/GDF15 axis is a critical driver of cancer cachexia. J Cachexia Sarcopenia Muscle. 2016;7(4):467-82.

75. Penafuerte CA, Gagnon B, Sirois J, Murphy J, MacDonald N, Tremblay ML. Identification of neutrophil-derived proteases and angiotensin II as biomarkers of cancer cachexia. $\mathrm{Br} \mathrm{J}$ Cancer. 2016;114(6):680-7.

76. Talbert EE, Lewis HL, Farren MR, Ramsey ML, Chakedis JM, Rajasekera P, et al. Circulating monocyte chemoattractant protein-1 (MCP-1) is associated with cachexia in treatmentnaive pancreatic cancer patients. J Cachexia Sarcopenia Muscle. 2018;9(2):358-68.

77. Hou YC, Wang CJ, Chao YJ, Chen HY, Wang HC, Tung HL, et al. Elevated Serum Interleukin-8 Level Correlates with Cancer-Related Cachexia and Sarcopenia: An Indicator for Pancreatic Cancer Outcomes. J Clin Med. 2018;7(12). 
78. Kuroda K, Nakashima J, Kanao K, Kikuchi E, Miyajima A, Horiguchi Y, et al. Interleukin 6 is associated with cachexia in patients with prostate cancer. Urology. 2007;69(1):113-7.

79. Mukherjee P, Mani S. Methodologies to decipher the cell secretome. Biochim Biophys Acta. 2013;1834(11):2226-32.

80. Welsh JB, Sapinoso LM, Kern SG, Brown DA, Liu T, Bauskin AR, et al. Large-scale delineation of secreted protein biomarkers overexpressed in cancer tissue and serum. Proc Natl Acad Sci U S A. 2003;100(6):3410-5.

81. Freire PP, Fernandez GJ, de Moraes D, Cury SS, Dal Pai-Silva M, Dos Reis PP, et al. The expression landscape of cachexia-inducing factors in human cancers. J Cachexia Sarcopenia Muscle. 2020;11(4):947-61.

82. Brandi J, Manfredi M, Speziali G, Gosetti F, Marengo E, Cecconi D. Proteomic approaches to decipher cancer cell secretome. Semin Cell Dev Biol. 2018;78:93-101.

83. Geyer PE, Holdt LM, Teupser D, Mann M. Revisiting biomarker discovery by plasma proteomics. Mol Syst Biol. 2017;13(9):942.

84. Makridakis M, Vlahou A. Secretome proteomics for discovery of cancer biomarkers. J Proteomics. 2010;73(12):2291-305.

85. Papaleo E, Gromova I, Gromov P. Gaining insights into cancer biology through exploration of the cancer secretome using proteomic and bioinformatic tools. Expert Rev Proteomics. 2017;14(11):1021-35.

86. Muralidharan-Chari V, Clancy JW, Sedgwick A, D'Souza-Schorey C. Microvesicles: mediators of extracellular communication during cancer progression. J Cell Sci. 2010;123(Pt 10):160311.

87. He WA, Calore F, Londhe P, Canella A, Guttridge DC, Croce CM. Microvesicles containing miRNAs promote muscle cell death in cancer cachexia via TLR7. Proc Natl Acad Sci U S A. 2014;111(12):4525-9.

88. Kim EY, Lee HY, Kim YS, Park I, Ahn HK, Cho EK, et al. Prognostic significance of cachexia score assessed by $\mathrm{CT}$ in male patients with small cell lung cancer. Eur J Cancer Care (Engl). 2018;27(1).

89. Vanhoutte G, van de Wiel M, Wouters K, Sels M, Bartolomeeussen L, De Keersmaecker S, et al. Cachexia in cancer: what is in the definition? BMJ Open Gastroenterol. 2016;3(1):e000097.

90. Martin L, Birdsell L, Macdonald N, Reiman T, Clandinin MT, McCargar L, et al. Cancer cachexia in the age of obesity: skeletal muscle depletion is a powerful prognostic factor, independent of body mass index. J Clin Oncol. 2013;31(12):1539-47.

91. Golse N, Bucur PO, Ciacio O, Pittau G, Sa Cunha A, Adam R, et al. A new definition of sarcopenia in patients with cirrhosis undergoing liver transplantation. Liver Transpl. 2017;23(2):143-54.

92. Portal D, Hofstetter L, Eshed I, Dan-Lantsman C, Sella T, Urban D, et al. L3 skeletal muscle index (L3SMI) is a surrogate marker of sarcopenia and frailty in non-small cell lung cancer patients. Cancer Manag Res. 2019;11:2579-88.

93. Prado CM, Lieffers JR, McCargar L, Reiman T, Sawyer MB, Martin L, et al. Prevalence and clinical implications of sarcopenic obesity in patients with solid tumours of the respiratory and gastrointestinal tracts: a population-based study. Lancet Oncol. 2008;9(7):629-35. 

Summary 
Cancer cachexia is a multifactorial syndrome that is characterized by an ongoing loss of skeletal muscle mass (with or without fat mass) that cannot be fully reversed by conventional nutritional support and that leads to progressive functional impairment. Among all cancer types, patients with pancreatic cancer have the highest prevalence of cachexia and often develop the most severe degrees of this syndrome. In cancer patients, cachexia is associated with reduced tolerance to anticancer treatment regimens, a marked reduction in quality of life, and significantly impaired overall survival. Therefore, management of the cachexia syndrome is likely to improve clinical outcome and quality of life of pancreatic cancer patients. However, despite many efforts of researchers and clinicians to improve our knowledge of cancer cachexia, this syndrome remains a significant medical problem as no clear standard of care exists.

Experimental preclinical systems used to model human cancer-associated cachexia, including traditional two-dimensional cell culture systems and animal models, have tremendously contributed to our current understanding of its pathophysiology. However, progress on the prevention, early diagnosis, and treatment of cancer cachexia is highly dependent on the successful translation of novel mechanistic findings into clinical benefits, which remains a major challenge. To gain better insight into the underlying mechanisms of human cancer-induced cachexia, there is an urgent need for better experimental preclinical models that closely recapitulate its key pathophysiological aspects.

New technological advances in cell culture have resulted in the development of the socalled organoid technology. Organoids are 3D structures grown from stem cells that consist of organ-specific cell types which self-organize into organ-like structures. Organoids can be efficiently established from patient-derived tissue, both healthy and tumor tissue, and retain important functional and structural characteristics of the organ of origin. This culture method provides a new platform for investigating the mechanisms underlying a disease in individual patients. In 2015, Boj et al. reported for the first time on the establishment of tumor organoids from patient-derived pancreatic tumor tissue. The high efficiency with which pancreatic tumor organoid cultures can be established from individual patients in combination with the thorough phenotyping of cachexia-related parameters of donor patients enables the application of organoids as a new experimental model in cachexia research.

The aim of the studies described in this thesis was to generate and characterize patientderived tumor organoids from well-phenotyped cachectic pancreatic cancer patients to provide a novel platform for studying the cachexia-inducing properties of pancreatic tumor cells and the mechanisms involved. 
In chapter 2, we described a systematic approach that was used to generate a pancreatic tumor organoid biobank. A comprehensive pre-operative patient assessment of cachexiarelated parameters (i.e. nutritional status, physical performance, body composition, and inflammation) was performed to assess the actual cachexia status of each individual patient in detail. We confirmed that the established pancreatic tumor organoids exhibited typical features of malignancy corresponding to the primary tumor. We also observed that these tumor organoids express variable levels of many cachexia-related factors, including $T N F-\alpha$, IL-6, IL-8, IL-1 $\alpha, I L-16, M c p-1, G D F 15$, and LIF. Importantly, tumor organoids from cachectic patients secreted more GDF15 and IL-8 compared to organoids from non-cachectic patients. We observed considerable interindividual variation in the production of these cachexiaassociated factors that was not necessarily in line with the cachexia status of the donor patient. These interindividual differences may be related to the heterogeneous presentation of cachexia.

Cancer cachexia is predominantly characterized by progressive weight loss with skeletal muscle loss, which can be the result of muscle atrophy. Muscle atrophy can be the consequence of an imbalance between processes that regulate protein synthesis and protein degradation. Cancer cachexia likely involves increased degradation rates of skeletal muscle proteins. In chapter 3, we investigated the direct effect of the tumor secretome on processes that regulate skeletal muscle mass. The secretome of both traditional two dimensional pancreatic tumor cell lines, of which the cachexia status of the donor patients is unknown, and pancreatic tumor organoids from a cachectic and a non-cachectic patient did not induce a significant upregulation of atrophy-related signaling pathways. These data do not support the hypothesis that tumor-derived factors activate catabolic signaling and/or inhibit protein synthesis in cultured muscle fibers.

Myogenesis is another important process that is involved in the maintenance of skeletal muscle mass. Myogenesis is the formation of muscular tissue by the fusion of myoblasts into multi-nucleated fibers called myotubes. In contrast to the hypothesis that pancreatic tumor organoid-derived factors induce muscle atrophy, we revealed that the tumor organoid secretome transiently increased the proliferation of myoblasts and accelerated their alignment and fusion. This enhanced myogenesis was accompanied by altered expression of factors governing muscle cell differentiation and a selective increase in muscle fiber type 2 , which is preferentially expressed in atrophy-sensitive glycolytic myofibers.

The involuntary loss of muscle mass in cancer cachexia mainly concerns skeletal muscle. Similar alterations have recently been observed in the cardiac muscle of cachectic patients, suggesting that these signaling pathways are also activated in cardiac muscle. So far, it is not known whether the changes found in atrophying skeletal and cardiac muscle in response to tumor-derived factors also occur in smooth muscle, the third muscle 
compartment that fulfils a crucial role in human physiology. Contractile SMCs are the forceproducing cells essential for gut motor activity required to move nutrients through the gastrointestinal (GI)-tract. Diminished contractile SMC function results in Gl-motility problems that may contribute to the underlying nutritional-related complications in cancer cachexia. In a recent study, it was shown that GI-related symptoms were more often identified in patients with cachexia compared to non-cachectic cancer patients. These symptoms could be secondary to Gl smooth muscle dysfunction.

To investigate whether tumor-derived factors also target contractile smooth muscle cells, we first developed a culture system which supports the maintenance of the contractile SMC phenotype in vitro (chapter 4). SMCs modulate uncontrollably towards a synthetic phenotype under standard cell culture conditions which lack the physiological microenvironment that normally keeps the cells in a contractile state. The synthetic phenotype is characterized by an increased proliferation rate, high migration rate, and pronounced synthesis of extracellular matrix (ECM) proteins. By careful optimization of the growth surface, serum concentrations, and cell-cell interactions, we developed a visceral SMC culture system in which SMCs can be kept in either a highly contractile state or modulated towards the synthetic phenotype in a controlled way. This simple and reproducible visceral SMC culture system may facilitate the identification of factors that drive visceral SMC phenotypic changes in health and disease.

In chapter 5, we exposed these contractile SMCs to tumor organoid-derived factors of cachectic and non-cachectic pancreatic cancer patients. We demonstrated that tumor organoid factors significantly reduced the expression of contractile SMC-specific proteins in both groups. These results indicate that tumor-derived factors negatively affect the contractile phenotype of SMCs, and are consistent with phenotypic modulation to the synthetic phenotype. In line, we demonstrated that the tumor organoid secretome of cachectic pancreatic cancer patients accelerates the proliferation of synthetic SMCs. The increase of the proliferation rate was accompanied by a significantly increased expression of the S100A4 gene, which is a marker of the synthetic proliferative SMC phenotype. Comparable results were observed in the intestinal smooth musculature of pancreatic cancer patients. We observed a significantly higher percentage of proliferative cells in the intestinal smooth muscle of pancreatic cancer patients with a low skeletal muscle mass, which is a key characteristic of cancer cachexia. These data imply that visceral contractile SMCs are also affected in cachectic patients and can undergo phenotypic modulation in response to tumor-derived factors.

In chapter 6, we retrospectively investigated key characteristics of the intestinal smooth musculature in a cohort of pancreatic cancer patients with and without a low skeletal muscle mass. We observed reduced contractile smooth muscle marker expression and fibrosis around the myenteric plexus, which provides motor innervation to the smooth musculature of the Gl-tract. These data suggest that both contractile function of smooth 
muscle cells and the regulation of their contractile functionality could be compromised in cachectic cancer patients.

Finally, in chapter 7, we discussed the implications of the main findings of this thesis and we defined recommendations for follow-up research. The studies described in this thesis demonstrated that the organoid technology is a novel platform that can be easily implemented in preclinical cancer cachexia research. Functional studies using the organoid secretome provided novel insights into the direct effect of tumor-derived factors on skeletal muscle cells. Furthermore, evidence collected in this thesis indicates that cachexiaassociated muscle wasting is not only limited to skeletal muscle. Both in vitro and retrospective cohort studies revealed cachexia-associated smooth muscle alterations.

Altogether, this novel preclinical organoid-based platform provides a valuable tool to increase our understanding of the mechanisms driving human cancer-induced cachexia. Expansion of the number of organoid cultures established from well-phenotyped patients will enable a more robust comparison of the differences between cachectic and noncachectic patients. 

Nederlandse samenvatting 
Cachexie is een multifactorieel syndroom dat wordt gekenmerkt door aanhoudend verlies van spiermassa (met of zonder verlies van vetmassa). Dit syndroom leidt tot progressieve functionele beperkingen en kan niet worden behandeld met gangbare voedingsinterventies. Cachexie komt veelvuldig voor bij patiënten met kanker, en wordt dan ook wel kankercachexie genoemd. De prevalentie van kankercachexie wordt sterk beïnvloed door het tumortype en stadium; het komt het vaakst voor bij patiënten met alvleesklierkanker. Kankercachexie is verder geassocieerd met een verlaagde tolerantie voor kankerbehandelingen en een sterk verminderde kwaliteit van leven. Vroegtijdige diagnose van kankercachexie en de ontwikkeling van nieuwe behandelingen tegen cachexie kunnen mogelijk de effectiviteit van kankerbehandelingen verbeteren en daarmee de levensduur en kwaliteit van leven van patiënten met kanker vergroten.

Om kankercachexie te kunnen voorkomen en behandelen is het essentieel om de onderliggende pathofysiologische mechanismen beter te begrijpen. In cachexie onderzoek wordt hiervoor veelvuldig gebruik gemaakt van experimentele modellen, waaronder traditionele twee dimensionale celkweek systemen en diermodellen. Deze modellen hebben enorm bijgedragen aan het verkrijgen van nieuwe inzichten. Echter, het verbeteren van de vroegtijdige diagnose en behandeling van kankercachexie is sterk afhankelijk van de succesvolle translatie van deze nieuwe basale bevindingen naar de kliniek. Eén van de belangrijkste tekortkomingen van de huidige experimentele kankercachexie modellen is dat deze niet een representatieve weergave zijn van het kankercachexie syndroom in de mens. Dit benadrukt de behoefte aan nieuwe preklinische modellen die de cachexie-kenmerken zoals die aanwezig zijn bij de patiënt beter reflecteren en die de heterogeniteit van de humane tumor beter reproduceren.

Nieuwe technologische ontwikkelingen hebben geleid tot de ontwikkeling van de zogenoemde organoïden technologie. Organoïden zijn zelf-organiserende driedimensionale structuren gekweekt vanuit stamcellen. Organoïden kunnen met hoge efficiëntie worden gekweekt uit weefsel van patiënten, zowel uit gezond weefsel als uit tumorweefsel, en behouden daarbij belangrijke functionele en structurele eigenschappen van het orgaan waaruit ze zijn gekweekt. Deze kweekmethode biedt onder andere de mogelijkheid om de moleculaire mechanismen die onderliggend zijn aan een ziekte te onderzoeken in individuele patiënten. In 2015 publiceerden Boj en collega's voor het eerst over het kweken van organoïden uit alvleesklier tumorweefsel van patiënten. De hoge efficiëntie waarmee alvleesklier tumor organoïden kunnen worden gemaakt van tumorweefsel verkregen uit individuele patiënten maakt deze technologie een potentieel zeer bruikbaar nieuw experimenteel model voor cachexie onderzoek.

Het doel van dit proefschrift was om tumor organoïden te genereren en karakteriseren van alvleesklierkankerpatiënten waarvan de cachexie status uitgebreid was onderzocht, om 
vervolgens deze organoïden in te zetten als een nieuw platform voor het bestuderen van cachexie-inducerende eigenschappen van alvleesklier tumorcellen en het ontraadselen van de onderliggende moleculaire mechanismen.

In hoofdstuk 2 beschrijven we de systematische aanpak die is gebruikt voor het opzetten van een alvleesklier tumor organoïden biobank. Patiënten waarbij de alvleeskliertumor operatief werd verwijderd ondergingen voorafgaand aan de operatie een uitgebreide screening van cachexie-gerelateerde parameters (o.a. gewichtsverlies, voedingsstatus, lichaamssamenstelling en bloedwaarden). Vervolgens werden van het operatief verwijderde tumorweefsel alvleesklier tumor organoïden gemaakt. Deze organoïden vertoonden typische kenmerken van maligniteit (o.a. histologisch en genetisch) die overeenkwamen met die van de primaire tumor. Daarnaast werden door deze tumor organoïden vele cachexie-gerelateerde genen in verschillende mate tot expressie gebracht, waaronder TNF- $\alpha, I L-6, I L-8, I L-1 \alpha, I L-16, M C p-1$, GDF15 en LIF. Het was interessant dat hogere concentraties GDF15 en IL-8 werden uitgescheiden door tumor organoïden van cachectische patiënten in vergelijking met organoïden van niet-cachectische patiënten. De interindividuele variatie die werd waargenomen in de mate van expressie van deze cachexie-geassocieerde factoren kwam niet altijd overeen met de cachexiestatus van de donorpatiënt. Deze bevindingen duiden op het behoud van de heterogene aspecten van kanker cachexie in het tumor organoïde model.

Kankercachexie wordt voornamelijk gekenmerkt door progressief gewichtsverlies en afbraak van skeletspieren, wat kan worden veroorzaakt door spieratrofie. Spieratrofie kan ontstaan door een verstoorde balans tussen processen die de spieropbouw en spierafbraak reguleren. Bij kankercachexie lijkt overwegend een verhoogde afbraak van spiereiwitten een rol te spelen. In hoofdstuk $\mathbf{3}$ hebben we het directe effect van factoren die worden uitgescheiden door de tumor, ook wel het tumor secretoom genoemd, onderzocht op processen die skeletspier massa reguleren. Zowel het secretoom van traditionele alvleesklier tumor cellijnen, waarvan de cachexie status van de donorpatiënten onbekend is, als het secretoom van alvleesklier tumor organoïden van een cachectische en nietcachectische patiënt veroorzaakte geen significante toename van atrofie-gerelateerde signaleringsmechanismen. Deze resultaten impliceren dat de factoren die worden uitgescheiden door alvleeskliertumorcellen niet direct spieratrofie induceren.

Een ander belangrijk proces dat betrokken is bij het behoud van spiermassa is myogenese. Myogenese is de vorming van spierweefsel door de fusie van myoblasten in meerkernige vezels, ook wel myotubes genoemd. In tegenstelling tot de verwachting dat factoren die worden uitgescheiden door de tumor spieratrofie zouden induceren, zagen we dat het secretoom van alvleesklier tumor organoïden een versnelde proliferatie van myoblasten veroorzaakte. De versnelde proliferatie ging gepaard met een versnelde fusie van deze myoblasten wat resulteerde in vroege vorming van myotubes. Dit was in 
overeenstemming met de verhoogde mRNA expressie van de fusiemarker Myomaker. De morfologische en transcriptionele veranderingen gingen verder gepaard met een verhoogde expressie van verschillende factoren die betrokken zijn bij belangrijke biochemische processen in gedifferentieerde spieren. De versnelde myogenese ging ook gepaard met een selectieve toename van type II spiervezels, ook wel fast-twitch glycolytische spiervezels genoemd. Eerdere studies suggereren dat deze glycolytische spiervezels gevoeliger zijn voor atrofie-inducerende factoren.

Het verlies van spiermassa bij kanker cachexie betreft voornamelijk de skeletspier. Vergelijkbare veranderingen zijn onlangs ook waargenomen in de hartspier van cachectische patiënten. Dit suggereert dat dezelfde signalering mechanismen ook in de hartspier geactiveerd kunnen worden. Tot dusver is het niet bekend of de veranderingen die aanwezig zijn in de skeletspier en de hartspier van cachectische patiënten ook aanwezig zijn in de gladde spier, het derde spiercompartiment van het menselijk spierstelsel. De contractiele functie van gladde spiercellen is essentieel voor de motorische activiteit van de darmen die nodig is om voedingsstoffen door het maagdarmkanaal te verplaatsen. Verminderde contractie van gladde spiercellen kan resulteren in motiliteitsproblemen die mogelijk kunnen bijdragen aan de onderliggende voeding-gerelateerde symptomen bij kankercachexie. In een recente studie is aangetoond dat maag-darm gerelateerde symptomen, zoals vroegtijdige verzadiging, diarree en obstipatie, vaker worden geïdentificeerd bij kankerpatiënten met cachexie in vergelijking met niet-cachectische kankerpatiënten. Deze symptomen kunnen secundair zijn aan gladde spierstoornissen in het maagdarmkanaal.

Om het directe effect van het tumor secretoom op contractiele gladde spiercellen te kunnen onderzoeken, hebben we eerst een kweek systeem ontwikkeld waarin humane viscerale gladde spiercellen in een contractiele toestand gekweekt kunnen worden (hoofdstuk 4). Onder standaard celkweek condities verliezen gladde spiercellen het contractiele fenotype en moduleren deze cellen naar het zogenoemde synthetisch fenotype, dat wordt gekenmerkt door een toename in de proliferatie en migratie en de synthese van extracellulaire matrix (ECM) eiwitten. Door een zorgvuldige optimalisatie van het groeioppervlak, de serum concentraties in het groeimedium en de cel-cel interacties hebben wij een eenvoudig en reproduceerbaar humaan glad spiercel kweeksysteem ontwikkeld. Deze geoptimaliseerde kweek condities maken het mogelijk om het fenotype van de gladde spiercel gecontroleerd te moduleren naar zowel het synthetische als het contractiele fenotype. Dit model kan bijdragen aan de identificatie van factoren die het fenotype van viscerale gladde spiercellen kunnen veranderen onder normale en pathofysiologische omstandigheden. 
In hoofdstuk 5 hebben we vervolgens deze contractiele gladde spiercellen blootgesteld aan het tumor organoïden secretoom van cachectische en niet-cachectische alvleesklierkankerpatiënten. We hebben aangetoond dat het tumor organoïden secretoom van beide patiëntgroepen de expressie van gladde spier-specifieke contractiele eiwitten significant verlaagde. De verminderde expressie van deze eiwitten in respons op de factoren uitgescheiden door de tumor wijst op een verlies van het contractiele gladde spiercel fenotype. Daarnaast hebben wij aangetoond dat het tumor organoïden secretoom van cachectische alvleesklierkankerpatiënten de proliferatie van synthetische gladde spiercellen versnelde. De toename in proliferatie ging gepaard met een significant verhoogde expressie van het S100A4 gen dat een marker is van het synthetisch proliferatieve gladde spiercel fenotype. Vergelijkbare resultaten werden waargenomen in het glad spierweefsel in de darmwand van alvleesklierkankerpatiënten. Een significant hoger percentage gladde spiercellen dat positief is voor de proliferatie-marker Ki-67 werd teruggevonden in het glad spierweefsel van alvleesklierkankerpatiënten met een lage skeletspiermassa, een parameter die is geassocieerd met kankercachexie. Deze data wijzen erop dat viscerale contractiele gladde spiercellen fenotypische modulatie ondergaan in respons op factoren die door de tumor van alvleesklierkankerpatiënten worden uitgescheiden.

In een retrospectief cohortonderzoek hebben we vervolgens specifieke karakteristieken van het contractiele glad spierweefsel van de darmwand onderzocht van alvleesklierkankerpatiënten met een hogere versus een lagere skeletspiermassa (hoofdstuk 6). Rondom de darmen bevinden zich twee lagen glad spierweefsel: aan de binnenkant zit de dwarslopende spierlaag en aan de buitenkant de longitudinale (over de lengte lopende) spierlaag. In de groep alvleesklierkankerpatiënten met een lage skeletspiermassa was er een significant verminderde expressie van het gladde spier-specifieke eiwit smootheline in de longitudinale spierlaag. Verlaagde expressie van het smootheline eiwit wijst mogelijk op een verminderde contractiele functie van het glad spierweefsel. Tegelijkertijd was er een significant verhoogde afzetting van het ECM-eiwit collageen zichtbaar rondom de myenterische plexus, ook wel het darmzenuwstelsel genoemd, dat zich tussen de dwarslopende en longitudinale spierlaag bevindt. Deze verhoogde collageenafzetting belemmert mogelijk de prikkeloverdracht tussen het darmzenuwstel en het glad spierweefsel en suggereert dat de regulatie van contractiele glad spierweefsel verstoord zou kunnen zijn.

Ten slotte hebben we in hoofdstuk 7 de implicaties van de belangrijkste bevindingen die in dit proefschrift beschreven staan besproken en hebben we aandachtspunten en aanbevelingen voor vervolgonderzoek gedefinieerd. De studies die in dit proefschrift staan beschreven tonen aan dat de organoïden technologie een nieuw platform is dat geïmplementeerd kan worden in preklinisch kankercachexie onderzoek. Functionele studies 
met het tumor organoïden secretoom leverden nieuwe inzichten op over het directe effect van factoren die worden uitgescheiden door tumorcellen op skeletspiercellen. Daarnaast hebben we aanwijzingen gevonden dat cachexie-gerelateerde spier problemen zich niet beperken tot skeletspieren, maar ook optreden in gladde spieren. Het nieuwe preklinische organoïde platform is daarmee een waardevol hulmiddel voor het verkrijgen van nieuwe inzichten in de mechanismen die bijdragen aan de ontwikkeling van kankercachexie. Uitbreiding van de organoïden biobank van kankerpatiënten waarvan de cachexie status uitgebreid is onderzocht is noodzakelijk om verschillen tussen cachectische en nietcachectische patiënten nog beter te kunnen vergelijken. 
Scientific and societal impact 


\section{Relevance}

Cancer cachexia is a severe wasting condition affecting $50-80 \%$ of all cancer patients. It is responsible for up to $20 \%$ of cancer-related deaths. Among all cancer types, patients with pancreatic cancer have the highest prevalence of cachexia and often develop the most severe degrees of this syndrome. Nevertheless, despite its high burden in cancer patients, cachexia still remains a relatively underestimated and underrecognized medical problem in the oncology field. No standard-of-care treatment is available for this syndrome and progress on the prevention and early diagnosis has only been marginal in the past decade. Given this, it is not surprising that the development of preventive and therapeutic strategies against cachexia is perceived as an urgent need by health professionals. Current research in the cachexia field is therefore predominantly committed to unraveling the underlying mechanisms that drive cancer-induced cachexia.

To date, pre-clinical studies have tremendously contributed to our current understanding of the pathophysiology of cachexia. However, progress on the prevention, early diagnosis, and treatment of cancer cachexia is highly dependent on the successful translation of novel mechanistic findings into clinical benefits. The translation of these findings has predominantly been hampered by the lack of preclinical models that closely recapitulate the pathophysiological aspects of this syndrome. Therefore, there is an urgent need for novel experimental preclinical models that can be used in cancer cachexia research.

\section{Organoid technology: a novel platform in translational cancer cachexia research}

This thesis demonstrated that the organoid technology can provide a novel platform in translational cancer cachexia research. A major advantage of this technology is that organoids can be efficiently generated from tumor resection material and can be maintained in culture while retaining characteristic malignant and pathophysiological aspects of the original tumor. Since the discovery of the organoid technology in 2009, numerous protocols have been developed for the successful generation of organoids from various cancer types, including cancer types that are often associated with cachexia (e.g. colorectal, lung, head and neck, pancreas, and gastro-esophageal cancer). In the future, the currently established pancreatic tumor organoid biobank may be expanded with tumor organoids originating from multiple cancer types. In combination with a comprehensive pre-operative patient assessment of cachexia-related parameters (i.e. nutritional status, physical performance, body composition, and inflammation), this platform provides new opportunities to explore the cachexia-inducing properties of patient-derived cancer cells and to link novel mechanistic findings to specific aspects of the actual cachexia status of the patient. 
A living tumor organoid biobank can be particularly useful to investigate which mechanisms are differentially activated in primary tumor cells from cachectic versus non-cachectic patients. Simultaneously, the tumor secretome of these organoid cultures can be used to identify the cachexia-inducing mediators that are released by these tumor cells. Gaining novel insights in these cachexia-inducing mechanisms and the identification of cachexiaassociated factors released by these tumor cells are essential to better understand the origin and pathophysiology of this wasting syndrome.

Nevertheless, although the work described in this thesis provides evidence that these tumor organoids retain cachexia-inducing properties in vitro, this work also revealed difficulties that are commonly encountered in translational cancer research. For example, considerable heterogeneity has been observed among the cachexia-related parameters, which greatly complicates the identification of strong associations between experimental data and clinical aspects of the actual cachectic status of these patients. To date, this is still one of the major issues that impede the translation of novel findings into clinical advances in the cancer cachexia field. Given the heterogeneous presentation of the cancer cachexia syndrome, expansion of the living organoid biobank from cancer patient of whom the cachexia status is thoroughly assessed, will therefore be crucial to identify clinically relevant associations between novel in vitro findings and cachexia-related parameters.

In addition, cancer cachexia is described to evolve through a spectrum that can generally be subdivided into three clinically relevant stages. Expansion of the number of organoid cultures can result in a better representation of the different stages of the cachexia syndrome within this living organoid biobank. In future studies, this knowledge may be used to identify diagnostic and prognostic biomarkers. These biomarkers can potentially be used to recognize this syndrome in the early stages of weight loss when multimodal interventions (i.e. nutritional and psychosocial support, physical exercise, cancer treatment, and management of toxicities of cancer treatment) are more effective, and to monitor disease progression, respectively.

The generation and initial characterization of pancreatic tumor organoids as a novel model to study cancer-induced cachexia has been described in a manuscript that is published in the Journal of Cachexia, Sarcopenia and Muscle (JCSM). This journal is an international journal dedicated to publish research that is related to cachexia and sarcopenia, as well as its physiological and pathophysiological changes in response to different illnesses, including cancer. This journal presents both basic and clinical research topics that benefit physicians, biochemists, biologists, dieticians, pharmacologists, and students. To further facilitate advances in translational cancer cachexia research, the organoid biobank will be made available for academic research.

\section{Tumor-muscle crosstalk in cancer cachexia}

The current research predominantly focuses on the functional impact of organoid-derived factors on various cell types implicated in the pathogenesis of cachexia. Given that skeletal 
muscle mass depletion is a key feature of cancer cachexia and contributes to progressive weight loss and a decline in functional abilities, the direct effect of tumor organoid-derived factors on skeletal muscle cells has been investigated in this thesis. Contrary to the general hypothesis that factors released from tumor cells promote muscle wasting by directly targeting skeletal muscle tissue, the human pancreatic tumor organoid secretome did not induce skeletal muscle atrophy signaling in cultured skeletal muscle fibers. Instead, pancreatic tumor organoid-derived factors transiently increased the proliferation of cultured myoblasts and accelerated their alignment and fusion into skeletal muscle fibers. This process was accompanied by altered expression of factors governing muscle cell differentiation and muscle fiber types.

These data imply that pancreatic tumor organoid-derived factors do not directly activate signaling mechanisms that induce muscle breakdown but rather affect other processes that are involved in the maintenance of muscle mass. Given that organoids promise greater representation of the tissue of origin when compared to the widely used traditional cell culture systems, this organoid model can potentially reveal novel insights into cancer-induced muscle alterations in humans. However, it is key to expand the number of organoid cultures to identify whether these alterations are associated with clinically relevant aspects of the actual cachexia status of these patients and to validate these results in skeletal muscle biopsies from cachectic and non-cachectic patients. In addition, these studies will also help to distinguish direct and indirect effects of tumor-derived factors on skeletal muscle (patho)physiology in relation to the cachexia status of the donor patient, thereby contributing to a better understanding of cachexia-inducing mechanisms.

Furthermore, this thesis also provides evidence that cachexia-associated muscle wasting not only affects skeletal muscle tissue but also smooth muscle. Impairment of the contractile function of the intestinal smooth musculature may contribute to the frequently observed gastrointestinal motility problems in these patients. The established human visceral smooth muscle cell $(S M C)$ culture system can greatly facilitate future studies to further explore the direct effects of tumor organoid-derived factors from cachectic and noncachectic patients on the SMC phenotype. However, data regarding GI-related symptoms and functionality of the Gl-tract in cachectic patients are lacking and should be a topic of future research. It is important to recognize that diminished contractile function of the intestinal smooth musculature may contribute to the underlying nutritional-related complications in cancer cachexia. Unrecognized GI-motility problems can have serious nutritional and metabolic consequences (i.e. dehydration, malnutrition, and weight loss). It is therefore key to recognize these Gl-related symptoms at early stages when nutritional and pharmacological interventions can prevent progression towards the more advanced cachectic stages. 
Dankwoord 
En dan tot slot, het dankwoord! Voor mij betekend dit dat ik bijna aan het eind ben gekomen van mijn promotietraject. Een gevoel van trots en opluchting gaan op dit moment door mij heen. Trots op het eindresultaat dat hier voor je ligt. Opgelucht dat het eindelijk zover is dat ik mijn proefschrift mag gaan verdedigen! Ik ben dankbaar voor alle hulp, steun en gezelligheid die ik heb gehad tijdens deze periode. Daarom wil ik in dit laatste hoofdstuk iedereen bedanken die op welke manier dan ook heeft bijgedragen aan de totstandkoming van mijn proefschrift.

Allereerst wil ik mijn promotieteam bedanken. Beste Steven, bedankt dat ik de mogelijkheid heb gekregen om mijn promotieonderzoek te doen op de afdeling Chirurgie. Wat heb ik hier een mooie tijd gehad! In tegenstelling tot het reguliere promotietraject heb ik tijdens mijn afstudeerstage van de master Biomedical Sciences een aanvraag geschreven voor het 'NUTRIM Graduate Programme'. Deze aanvraag gaf mij de mogelijkheid om mijn eigen onderwerp, onderzoeksvoorstel en begeleiders te bepalen. En dit heeft goed uitgepakt! Ik werd uitgenodigd voor een interview waar ik mijn voorstel heb mogen presenteren en toelichten, en met succes, het voorstel werd geselecteerd! Je zei altijd dat dit 'mijn eigen bedrijfje is, mijn eigen BV' en zo zag je het ook! Je hebt me de vrijheid gegeven in het opzetten van mijn eigen onderzoeksprojecten en dankzij jouw support was het voor mij mogelijk om een uniek model op te zetten in het lab, de pancreas tumor organoïden. Ik ben je dan ook enorm dankbaar voor de kansen en mogelijkheden die je mij hebt gegeven! Naast onderzoek was er ook altijd tijd voor gezelligheid. Publicaties werden samen gevierd en elk jaar was er weer die overheerlijke barbecue met al je PhD studenten bij jou in de achtertuin.

Beste Sander, wat heb ik ongelooflijk veel van je geleerd in de afgelopen jaren! Ik wil je dan ook bedanken voor de vele uren die je hebt gestoken in mijn promotietraject. Als dagelijks begeleider stond je deur altijd voor mij open. Of het nu ging om de planning van nieuwe experimenten door te bespreken, om te brainstormen over nieuwe projecten, om je met veel enthousiasme nieuwe data te laten zien, gewoon een gezellig praatje, of om even mijn hart bij je te kunnen luchten. Het maakte voor jou niet uit, je maakte hier altijd tijd voor. Dat heb ik altijd enorm gewaardeerd! Ook wil ik je bedanken voor alle uren die je hebt gestoken in het kritisch lezen van mijn geschreven stukken. Jouw oog voor detail en de constructieve feedback die ik altijd van je terugkreeg, hebben er niet alleen voor gezorgd dat de kwaliteit van deze stukken omhoogging, maar ook dat ik hier zelf veel van heb geleerd! Dit zal ik altijd met mij meenemen, dankjewel daarvoor.

I also would like to thank the members of the thesis assessment committee, Prof. dr. Peter Peters, Prof. dr. Annemie Schols, Prof. dr. Daisy Jonkers, Prof. dr. Thorsten Cramer and Dr. Marie-Luce Bochaton-Piallat. I appreciate their time critically reading and evaluating my thesis. 
Of course, this thesis would not have been possible without the contribution of all the coauthors and the many collaborators! Thank you all for the scientific discussions about the study/experimental designs, your help with the experiments, and all your suggestions and feedback on the papers/chapters!

Beste Annet, beste Mirjam, bedankt dat jullie mijn paranimfen willen zijn! Mirjam, we zijn tegelijkertijd begonnen aan het promotietraject en je was mijn 'kantoormaatje' vanaf de eerste dag. Elke dag weer hadden we elkaar altijd weer veel te vertellen. Het werd vaak al snel iets te gezellig, maar als het nodig was, hadden we ook niet veel woorden nodig om aan te geven dat er toch echt nog gewerkt moest worden. Uiteraard moest er dan wel eerst nog een koffie moment voor later gepland worden om weer volop verder te kunnen kletsen! Ook al werkte we allebei in een andere onderzoeksgroep, ik heb het altijd fijn gevonden dat we alles met elkaar hebben kunnen delen; lab-issues, phd-issues, frustraties, successen en overwinningen. Dankjewel daarvoor! Ook jij bent nu bezig met het afronden van je proefschrift. De laatste loodjes! Ik wens je nog heel veel succes daarmee. Hopelijk blijven we elkaar ook hierna nog vaak zien en kunnen we onder het genot van een heerlijk wijntje samen terugkijken op de afgelopen jaren. Annet, ook jou wil ik bedanken voor de fijne samenwerking en gezelligheid! Toen jij aan je promotietraject begon, heb je je 'plekkie' gekregen bij Mirjam en mij op kantoor. Zo kon je ook gelijk wennen aan de 'Limburgse taal', want af en toe was het toch echt niet te begrijpen, laat staan uit te spreken. Een ding is nu in ieder geval duidelijk, het is niet 'Margráten' maar 'Màrgraten'! Ik wens jou nog heel veel succes met je studies en het afronden van je promotietraject. Dat gaat zeker weten helemaal goed komen!

Ook wil ik al mijn (PhD-to-be) collega's van de cachexie-groep bedanken, David, Jorne, Merel, Gregory, Marjolein en Min. David, jij bent als eerste begonnen met het doen van cachexie onderzoek onder begeleiding van Steven en Sander. Ondertussen is de groep alleen maar groter geworden en lopen er op dit moment verschillende klinische en translationele studies! Jouw klinische kennis en ervaring hebben mij enorm geholpen bij het verder uitzetten van mijn onderzoeksprojecten. Dankjewel daarvoor! Jorne, we hebben elkaar leren kennen op het cachexie congres in Berlijn. Nog niet wetende dat jij ook onder begeleiding van Sander lab-experimenten zou komen doen. Niet veel later zaten we samen op het lab skeletspier cellen te kweken. Ik heb erg genoten van onze samenwerking! Jouw vrolijkheid, jouw humor en de vele mooie verhalen die je altijd te vertellen had zorgde ervoor dat het altijd gezellig was. Niet alleen in het lab, maar ook tijdens de koffiepauzes, borrels, congressen en bbq's. Ook jij bent nu bijna klaar met het afronden van je proefschrift. Ik wens je nog heel veel succes met de laatste loodjes! Merel, ik kan me nog goed herinneren dat je bij me langs kwam als student; je had grootse plannen! Je ging naar de 'Tuveson lab' in New York om te leren om pancreas tumor organoïden te kweken en om de gevoeligheid van chemotherapie op deze organoïden te onderzoeken. En met succes! Je 
bent teruggekomen om je onderzoek bij de afdeling Chirurgie verder af te ronden om vervolgens aan je promotieonderzoek te starten op dit onderwerp. Het was fijn om iemand erbij te hebben die ook pancreas tumor organoïden is gaan kweken! Naast onze samenwerking op heb lab, heb jij, met je klinische achtergrond, mij ook enorm veel geholpen met het analyseren van de CT-scans en met het aanvullen van de klinische database. Dankjewel daarvoor! Naast onze samenwerking, hebben we samen ook vele leuke momenten beleefd. Onder andere samen op congres in Rome, Genève en Maastricht; de details laat ik voor nu achterwege... maar wat was het gezellig met jou! Gregory, ook jij bedankt voor de fijne samenwerking! Marjolein, hoewel we niet hebben samengewerkt op dezelfde projecten, wil ik je evengoed bedanken voor al je hulp met de klinische database. Bedankt dat ik met al mijn vragen hierover bij je terecht kon. Min, newest member of the cachexia research group. You are a very kind person and it was always a pleasure working with you. I wish you all the best and good luck with your projects, I am convinced you will succeed!

Ook wil ik al mijn (mede-PhD) collega's bedanken voor de fijne samenwerking en de gezellig sfeer op de afdeling in de afgelopen jaren. Rob, Sara, Loes, Janine, Cathelijne, Romy, Evie, Caitlin, Kim, Renée, Aurelia, Anne, Kees, Jos, Patrick, Jacqueline, Sebastiaan, Remon, Tom, Ilse, Kiran, Claire, Dennis, Junfang, Lin, Xinwei, Hong, Gloria, Lara, Anjali, Zita en Ralph: bedankt voor de gezelligheid tijdens de koffie, lunch, borrels bij Thembi, sinterklaasborrels, kerstborrels, en labuitjes.

Kaatje, Frank en Hans, bedankt voor jullie feedback die jullie mij hebben gegeven tijdens de wekelijkse vrijdagochtend bespreking en het meedenken met mijn onderzoeksprojecten.

Natuurlijk ook een groot dankjewel voor de analisten! Zonder jullie hulp was het zeker niet gelukt om alle studies te kunnen doen zoals die nu in dit proefschrift staan beschreven. Bas, wat heb ik ongelooflijk veel gehad aan al je hulp! Of het nu ging om het snijden van coupes, IHC/IF kleuringen, microscopie, qPCR analyses, celkweek, of organoïden kweek, je stond altijd klaar om te helpen! Ook kon ik altijd met mijn vragen bij je terecht. Of ja, hiervoor hoefde ik natuurlijk ook alleen maar een deur verder binnen te lopen dan mijn eigen kantoor dus was er ook geen ontkomen aan! Daarnaast was je ook altijd wel in voor een praatje en heb ik altijd met veel plezier met je samen gewerkt, dankjewel daarvoor! Mo, ook jou wil ik bedanken voor de plezierige samenwerking! Ik heb veel geleerd van jouw kennis en ervaring in de celkweek. Ook ben je ook dankbaar voor alle tips, zowel op wetenschappelijk als persoonlijk vlak, die je me de afgelopen jaren hebt gegeven. Annemarie, of het nu ging om Western Blots, RNA isolaties of ELISA's, ik kon dit altijd met een gerust hart aan jou overlaten. De zorgvuldigheid en nauwkeurigheid waarmee jij deze analyses uitvoert, hebben mij enorm geholpen om data te verkrijgen van de vele verzamelde samples. Dankjewel voor alle hulp die ik van je heb mogen krijgen tijdens mij 
promotietraject! Cathy, wat vond ik het leuk dat we collega's van elkaar werden nadat we samen onze afstudeerstage hadden afgerond op de afdeling Chirurgie! Ook jij hebt me ongelooflijk veel geholpen tijdens mijn promotietraject; de vele RNA isolaties en qPCR analyses die je voor me hebt gedaan en natuurlijk ook al je hulp met het verzamelen van patiënten materiaal op de OK en bij de Pathologie afdeling. Ik wil je bedanken voor jou inzet en voor de gezellige en fijne samenwerking! Chantal, als je tussen je andere werkzaamheden door nog tijd over had, stond ook jij altijd klaar om te helpen. Dankjewel voor je hulp in het lab en alle gezelligheid tijdens de lunchpauzes!

Dan zijn er natuurlijk ook nog de studenten die bij mij stage hebben gelopen en hebben meegewerkt aan mijn promotieonderzoek. Linda, Liesbeth, Anda, Tessa, Ryan en Britta Bedankt voor al jullie inzet en jullie bijdrage aan mijn studies!

Tessa, jou wil ik hier nog in het bijzonder bedanken. Wat heb je me ongelooflijk veel geholpen de afgelopen jaren! Hoe had ik dit allemaal zonder jou moeten doen? Je was begonnen als derdejaars student op de afdeling chirurgie en al snel ging je meewerken op een van mijn onderzoeksprojecten. Je was zo enthousiast dat je ook graag je afstudeerstage bij mij zou willen komen doen. Dan regelen we dat natuurlijk! Het was altijd fijn om met je samen te werken, we wisten precies wat we aan elkaar hadden, en bovenal, wat hebben we samen ook veel gelachen! Wat was ik trots op je tijdens je afstudeerzitting en blij dat je daarna mijn collega werd, 'chief organoids'. Ik kan niet vaak genoeg zeggen hoe dankbaar ik ben voor je hulp in het lab en de leuke momenten die we samen hebben beleefd.

Livia, ook jij bedankt voor al je administratieve hulp! Je stond altijd klaar om te helpen en was altijd wel in voor een gezellig praatje. En natuurlijk niet te vergeten, de 'planking challenge', je was de beste! Ondertussen heb je de afdeling chirurgie verlaten en ben je begonnen aan een nieuwe uitdaging. Ik wens je heel veel succes en plezier op je nieuwe werkplek! Nicole, ook jij bedankt voor al je administratieve hulp tijdens het afronden van mijn promotietraject. Door de coronamaatregelen hebben we elkaar helaas nog niet gezien, maar zodra het weer kan, zal ik zeker eens bij je binnen lopen!

Ramon, vanaf het begin was je al betrokken bij een van mijn onderzoeksprojecten. Ik wil je bedanken voor het delen van je expertise in de regulatie van spiermassa, je waardevolle input op de data van de skeletspier celkweek experimenten en de tijd die je hebt gestoken in het kritisch lezen van het manuscript dat hieruit is voortgekomen. Ik heb altijd met veel plezier met je samengewerkt, dankjewel daarvoor! Marco, bedankt voor je hulp en ondersteuning met de skeletspier celkweek experimenten. Wouter en Wessel, ook wil ik jullie bedanken voor de hulp die ik van jullie heb gekregen. Wanneer ik weer het Pulmonologie lab binnen liep, waren jullie altijd bereid om mij te helpen. Dankjulliewel! 
Ook wil ik mijn collega's bij Maastro, Maastro-lab en Precision Medicine bedanken. In het bijzonder Dirk en Marc, bedankt voor jullie steun en het vertrouwen dat jullie mij hebben gegeven voor het afronden van mijn proefschrift en als post-doc onderzoeker binnen jullie onderzoeksgroep.

Voor een goede afwisseling tussen werk en ontspanning, wil ik ook mijn vrienden bedanken. Lean, al vriendinnen van kleins af aan en dat is altijd zo gebleven! Ook al wonen we niet bij elkaar in de buurt, het is altijd weer gezellig met jou. Ondanks de afstand, staan we altijd voor elkaar klaar. Of het nu gaat om gewoon weer even bij te kletsen of om even ons hart bij elkaar te luchten. Wat ben ik blij met jou als mijn vriendin! Arlette, Nicole en Eveline, dames, wat hebben we samen vele mooie momenten beleefd! We hebben elkaar leren kennen bij de studentenvereniging 'Volupia' en wat hebben we hier samen een mooie tijd gehad! Ook de vele etentjes, borrels, feestjes en de weekendjes weg in de afgelopen jaren hebben zeker bijgedragen aan de nodige ontspanning. Pauline, a.k.a. Soos! We hebben elkaar leren kijken tijdens de HBO-opleiding op Zuyd Hogeschool, we zijn daarna allebei de master Biomedical Sciences gaan doen en vervolgens zijn we beide gestart aan een promotietraject. leder z'n eigen weg, maar ondanks dat jij in Canada zat, hebben we altijd contact gehouden. 'Even' met jou bellen staat voor meer dan een uur gezelligheid en weer even lekker bijpraten over van alles en nog wat als we elkaar al weer veel te lang niet hadden gesproken. Ik hoop dat we elkaar nog vaak zullen spreken en wie weet, kunnen we elkaar binnenkort ook weer vaker zien! Glenn, nadat we elkaar hebben lekker kennen tijdens de master Biomedical Sciences, zijn we tegelijkertijd begonnen aan ons promotietraject. Ik kijk met veel plezier terug op de koffie/lunch pauzes; wanneer we weer even konden bij praten, frustraties konden delen, en samen hebben kunnen lachen om van alles en niets. Bedankt voor alle steun, het delen van jouw ervaringen en natuurlijk ook de fijne vriendschap. Ik wens jou veel succes met je nieuwe uitdaging en vooral ook heel veel woon plezier in Nieuwegein. Zoals we altijd tegen elkaar zeggen, we houden contact!

Daarnaast wil ik graag mijn schoonouders bedanken, Huub en Ria. Bedankt dat jullie altijd voor Roel en mij klaar staan!

Mijn twee broers, Martijn en Erwin. Ook wil ik jullie bedanken voor de steun die jullie mij hebben gegeven in de afgelopen jaren. Ook al wonen we niet bij elkaar in de buurt, we maken er altijd weer een leuke dag van als we bij elkaar zijn. Samen uiteten, gezelschapsspellen spelen, of er samen op uit. Bedankt voor al deze mooie momenten. Dat er nog vele mogen volgen!

Pap en mam, ook jullie wil ik bedanken voor alle steun en het vertrouwen die ik altijd van jullie heb gekregen. Het was voor jullie misschien niet altijd duidelijk waar ik mee bezig was, maar jullie leefden wel altijd met mij mee. Ik ben jullie enorm dankbaar voor de ruimte die 
jullie mij altijd hebben geven om te doen wat ik wilde doen. Dit heeft eraan bijgedragen dat ik sta waar ik nu sta en daar ben ik trots op. Dankjulliewel pap en mam!

Roel, de laatste woorden van mijn dankwoord zijn voor jou. Ook jou wil ik hier bedanken, want zonder jou was het mij nooit gelukt om tot dit eindresultaat te komen! Jij staat altijd voor mij klaar en laat mij inzien dat er veel belangrijkere dingen zijn dan werk. Als ik er even doorheen zat, was jij diegene die ervoor zorgde dat ik mijn hoofd weer helemaal leeg kon maken om daarna weer met nieuwe energie ertegenaan te kunnen gaan. Ook al was het voor mij niet altijd gemakkelijk om hier gelijk aan toe te geven, als snel genoot ik volop van de vele wandelingen en mountainbike tochten die wij samen hebben gemaakt en de spontane weekendjes weg. Samen genieten stond dan voorop! Voor jou was het ook zeker niet altijd gemakkelijk. Als ik weer een deadline had, in het weekend naar het lab moest voor mijn experimenten, weer weg was op congres of als ik in de avonduren weer bezig was met het afronden van mijn proefschrift. Ondanks alles heb je mij altijd gesteund. Nu mijn promotietraject bijna ten einde is, wordt het hoog tijd dat wij, samen met Bram, nog meer gaan genieten en samen nieuwe avonturen gaan beleven! 

Curriculum Vitae 



\section{About the author}

Rianne Vaes was born on September $24^{\text {th }} 1992$ (Helden, The Netherlands). After she completed her senior general secondary education (HAVO) at the Philips van Horne SG in Weert in 2009, she started the bachelor study Biology and Medical Laboratory Research at Zuyd University of Applied Sciences (Heerlen, The Netherlands). During her bachelor study, she performed her graduation internship at the Institute of Biochemistry and Molecular Biology (Uniklinik Aachen, Germany) under the supervision of Dr. Jörg Hartkamp. In 2013, Rianne successfully obtained her Bachelor's degree in Applied Sciences Cum Laude. In the same year, she has been awarded with the Zuyd thesis Award for the best bachelor thesis of Zuyd University.

In September 2013, she started with the 2-year master study Biomedical Sciences at Maastricht University to continue her education in the biomedical research field. During the first year, she performed her junior internship at the Department of Radiation Oncology (Maastricht University) under the supervision of Dr. Ludwig Dubois and Dr. Marike van Gisbergen. In 2014, Rianne started her senior master internship at the Department of Surgery at Maastricht University under supervision of Dr. Sander Rensen. During this internship, she studied the effect of pancreatic tumor cell-derived mediators on skeletal muscle atrophy signaling pathways. In 2015, Rianne obtained her master's degree.

After obtaining her master's degree, she started her PhD trajectory on pancreatic cancer cachexia at the Department of Surgery within NUTRIM School of Nutrition and Translational Research in Metabolism under supervision of Prof. Steven Olde Damink and Dr. Sander Rensen. Her PhD trajectory was funded by the NUTRIM Graduate Programme. The main focus of her $\mathrm{PhD}$ research was to generate and characterize patient-derived tumor organoids from well-phenotyped cachectic pancreatic cancer patients to provide a novel platform for studying cachexia-inducing properties of pancreatic tumor cells and the mechanisms involved. During her PhD-trajectory, she presented at several national and international conferences and was awarded with the best poster award at the NUTRIM Symposium 2016, Maastricht, The Netherlands. In 2019, she received the Young Investigators Award at the $12^{\text {th }}$ annual international Cachexia Conference in Berlin, Germany.

Rianne will continue her academic career as a postdoctoral researcher at the Department of Radiation Oncology (MAASTRO), Maastricht University Medical Center, Maastricht. Her research focuses on the identification of radiotherapy and immunotherapy-induced immune changes in lung cancer patients. 



\section{List of publications}




\section{Publications}

Vaes RDW, Welbers TTJ, van Dijk DPJ, Rennspiess D, zur Hausen A, Olde Damink SWM, Rensen SS. Intestinal smooth muscle aberrations in pancreatic cancer patients with sarcopenia. JCSM Rapid Communications. 2021 (in press)

Julian Palzer, Benedikt Mues, Richard Goerg, Merel Aberle, Sander S. Rensen, Steven W.M. Olde Damink, Rianne D.W. Vaes, Thorsten Cramer, Thomas Schmitz-Rode, Ulf P. Neumann, Ioana Slabu, Anjali A. Roeth. Magnetic fluid hyperthermia as treatment option for pancreatic cancer cells and pancreatic cancer organoids. Int J NanoMedicine. 2021 (in press)

Vaes RDW, van Dijk DPJ, Welbers TTJ, Blok MJ, Aberle MR, Heij L, Boj SF, Olde Damink SWM, Rensen SS. Generation and initial characterization of novel tumour organoid models to study human pancreatic cancer-induced cachexia. Journal of Cachexia, Sarcopenia and Muscle. 2020;11(6):1509-1524

Vaes RDW, van den Berk L, Boonen B, van Dijk DPJ, Olde Damink SWM, Rensen SS. A novel human cell culture model to study visceral smooth muscle phenotypic modulation in health and disease. American Journal of Physiology and Cell Physiology. 2018;315(4):C598-C607

van Dijk DPJ, Bakers FCH, Sanduleanu S, Vaes RDW, Rensen SS, Dejong CHC, Beets-Tan RGH, Olde Damink SWM. Myosteatosis predicts survival after surgery for periampullary cancer: a novel method using MRI. HPB. 2018;20(8):715-20

\section{Publications in preparation}

Vaes RDW, van Dijk DPJ, Olde Damink SWM, Rensen SS, Langen R. Human pancreatic tumor organoid-derived factors enhance myogenic differentiation. Submitted to Journal of Cachexia, Sarcopenia and Muscle (under revision)

Rianne D.W. Vaes, Lizza E.L. Hendriks, Marc Vooijs, Dirk De Ruysscher. Biomarkers of radiotherapy-induced immunogenic cell death. Submitted to Cells (under revision)

Jorne Ubachs, Wouter R.P.H. van de Worp, Rianne D.W. Vaes, Kenneth Pasmans, Ramon Langen, Ruth C.R. Meex, Sandrina Lambrechts, Toon van Gorp, Roy F.P.M. Kruitwagen, Steven W.M. Olde Damink, Sander S. Rensen. Ovarian cancer ascites induces skeletal muscle wasting in vitro and reflects sarcopenia in patients. Submitted to Journal of Cachexia, Sarcopenia and Muscle (under review) 
Vaes RDW, van Bijnen AA, Olde Damink SWM, Rensen SS. Pancreatic tumor organoidderived factors of patients with cachexia promote phenotypic modulation and proliferation of visceral smooth muscle cells.

Brenda Bakker, Rianne D.W. Vaes, Merel R. Aberle, Tessa T.J. Welbers, Thomas Hankemeier, Sander S. Rensen, Steven W.M. Olde Damink, Ron M.A. Heeren. Preparing ductal epithelial organoids for high spatial resolution molecular profiling using mass spectrometry imaging.

Merel Aberle, Rianne D.W. Vaes, Ludwig J. Dubois, Natasja G. Lieuwes, Rianne Biemans, Wouter van de Worp, Ramon Langen, Frederik-Jan van Schooten, Ronald van Dam, Steven Olde Damink, Sander S. Rensen. Novel models of human pancreatic cancer cachexia heterogeneity by transplantation of tumor organoids into mice: a pilot study. 
